\title{
A mathematical model of the dynamics of the left ventricle and the coronary circulation
}

Citation for published version (APA):

Arts, M. G. J. (1978). A mathematical model of the dynamics of the left ventricle and the coronary circulation. [Doctoral Thesis, Maastricht University]. Rijksuniversiteit Limburg. https://doi.org/10.26481/dis.19780519ma

Document status and date:

Published: 01/01/1978

DOI:

10.26481/dis.19780519ma

Document Version:

Publisher's PDF, also known as Version of record

\section{Please check the document version of this publication:}

- A submitted manuscript is the version of the article upon submission and before peer-review. There can be important differences between the submitted version and the official published version of record. People interested in the research are advised to contact the author for the final version of the publication, or visit the DOI to the publisher's website.

- The final author version and the galley proof are versions of the publication after peer review.

- The final published version features the final layout of the paper including the volume, issue and page numbers.

Link to publication

\footnotetext{
General rights rights.

- You may freely distribute the URL identifying the publication in the public portal. please follow below link for the End User Agreement:

www.umlib.nl/taverne-license

Take down policy

If you believe that this document breaches copyright please contact us at:

repository@maastrichtuniversity.nl

providing details and we will investigate your claim.
}

Copyright and moral rights for the publications made accessible in the public portal are retained by the authors and/or other copyright owners and it is a condition of accessing publications that users recognise and abide by the legal requirements associated with these

- Users may download and print one copy of any publication from the public portal for the purpose of private study or research.

- You may not further distribute the material or use it for any profit-making activity or commercial gain

If the publication is distributed under the terms of Article $25 \mathrm{fa}$ of the Dutch Copyright Act, indicated by the "Taverne" license above, 


\section{A MATHEMATICAL MODEL OF THE DYNAMICS OF THE LEFT VENTRICLE AND THE CORONARY CIRCULATION}

PROEFSCHRIFT

ter verkrijging van de graad van doctor in de geneeskunde aan de Rijksuniversiteit Limburg, on gezag van de Rector Magnificus Dr. H. A. Tiddens, hoogleraar in de fakulteit der geneeskunde, volgens besluit van het college van dekanen in het openbaar te verdedigen op vrijdag 19 mei 1978 des namiddags om vier uur precies, in de aula der universiteit.

door

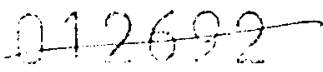

door

\section{Matheus Gijsbertus Jozef Arts}




$$
\begin{aligned}
\text { Promotoren : Prof. Dr. R.S. Reneman } \\
\text { Prof. Dr. P.C. Veenstra }
\end{aligned}
$$

Co-referenten : Prof. Dr. J.D. Laird

Dr. N. Westerhof

Een dee 1 van de dierexperimenten werden uitgevoerd in het laboratorium voor Cardiovasculair Onderzoek van Janssen Pharmaceutica te Beerse (België).

Deze onderzoekingen werden voor een belangrijk gedeelte uitgevoerd binnen de werkgemeenschap Hartfunctie van de Stichting voor Medisch Wetenschappelijk Onderzoek FUNGO met belangrijke financiële steun van de Nederlandse Organisatie voor Zuiver Wetenschappelijk Onderzoek (ZWO).

Het verschijnen van dit proefschrift werd mede mogelijk gemaakt door steun van de Nederlandse Hartstichting. 
mede dank zij Jacqueline Houter

Martine 



\section{CONTENTS}

\section{CONTENTS}

Chapter 2. BASIC ANATOMY AND PHYSIOLOGY OF THE HEART AND THE CORONARY ARTERIES

Chapter 3. DESIGN OF A MATHEMATICAL MODEL OF THE LEFT VENTRICLE 15

3.1. Introduction 15

3.2. Simplifications concerning the geometry of the heart 17

3.2.1. Consideration of the left ventricle 17

3.2.2. Rotational symmetry of the left ventricle 18

3.2.3. The cylindrical geometry of the left ventricle 18

3.3. Simplifications concerning the properties of the myocardial wall 20

3.3.1. The mechanical properties of the myocardial tissue 20

3.3.2. The fiber orientation in the myocardial wall 21

3.3.3. The mechanical properties of the endocardium 21

3.3.4. The sequence of the onset of contraction in the myocardium

3.4. The hemodynamic loading of the left ventricle 22

3.4 .1 . The aortic valve 22

3.4.2. The aortic input impedance 23

3.4.3. The left ventricular filling 24

3.5. The coronary circulation 24

3.5 .1 . The volume of the intramyocardial coronary

3.5.2. The large coronary arteries (I.D. $>400 \mu \mathrm{m}) \quad 24$

3.5.3. Vasoactivity 25

3.5.4. The coronary arterioles, capillaries and
venules

Chapter 4. CHARACTERIZATION OF THE MODEL BY A SYSTEM OF DIFFERENTIAL EQUATIONS

4.1. Introduction 28

4.2. Characteristic quantities of the instanteous situation 29

4.3. Some important related quantities 30

4.4. The computation of the derivatives of the characteristic quantities 
Chapter 5. THE STRESS-STRAIN RELATION OF THE MYOCARDIAL MATERIAL

5.1. Introduction

5.2. Approach to describe the mechanical properties of myocardial material

5.3. Model of the stress-strain-strainrate-time relation of cardiac muscle

5.3.1. Introduction

5.3.2. The dependence of force per sarcomere volume on sarcomere length, velocity of sarcomere shortening and time

5.4. Computer simulation of experiments on cardiac muscle 45

5.5. Isotropic myocardial material 49

5.6. Anisotropic myocardial material 52

5.7. Influence of isotropy or anisotropy of myocardial material on the systolyc pressure-volume relation of the left ventricle

Chapter 6. CHARACTERISTICS OF THE CORONARY CIRCULATION

6.1. Introduction

6.2. Physical properties of the large coronary arteries in dogs 61 6.2.1. Introduction

6.2.2. Methods

6.2.3. Results

6.2.4. Discussion

6.3. The coronary microcirculation

6.4 Mathematical representation of the coronary circulation

Chapter 7. DETERMINATION OF THE INSTANTANEOUS STRESSES AND STRAINS IN THE WALL OF THE CYLINDER

7.1. Introduction

7.2. Determination of dimensions and torsion angle of each subcylinder

7.3. Computation of the stresses in a subcylinder

7.4. Equilibrium of force and torque acting on the upper surface of the thickwalled cylinder

7.5. Calculation of local intramyocardial pressures and left ventricular pressure

Chapter 8. CALCULATION OF ADDiTIONAL RELEVANT QUANTITIES

8.1. Introduction

8.2. Calculation of aortic volume flow and aortic pressure

8.3. Calculation of the mechanical specific energy, generated during the cardiac cycle 
8.4. Calculation of the local coronary perfusion fraction in one cardiac cycle

Chapter 9. INPUT DATA AND INITIAL CONDITIONS OF THE PRESENT MODEL 102

9.1. Introduction 102

9.2. Input data of the present model 102

9.2.1. The geometry of the left ventricle 102

9.2.2. The fiber orientation across the myocardial wall 105

9.2.3. The sarcomere length across the myocardial wall 105

9.2.4. Velocity of the depolarization wave front across
the myocardial wall
107

9.2.5. The input impedance of the aorta 107

9.2.6. Hemodynamic quantities 109

9.2.7. The endocardium and other structures in the left 109

9.2.8. Properties of the large coronary arteries

( $1.0 .>400 \mathrm{jm}$ ) 110

9.2.9. Properties of the coronary microcirculation 111

9.3. Initial conditions 113

9.3.1. The hemodynamics of the left ventricle and aorta 114

9.3.2. Muscle mechanics 114

9.3.3. The large coronary arteries (I.D. > $400 \mu \mathrm{m}$ ) 115

9.3.4. The coronary microcirculation 115

Chapter 10. RESULTS 119

10.1. Introduction 119

10.2. Simulation of the cardiac cycle with parameter values $\begin{array}{ll}\text { as discussed in the previous chapters } & 119\end{array}$

10.3. Adaptation of several parameters in order to obtain a more realistic simulation of the cardiac cycle 128

10.4. Simulation of deviant cardiac cycles 131

Chapter 11. DISCUSSION 143

11.1. Introduction 143

11.2. Simulation of a normal cardiac cycle 143

11.2.1. Introduction 143

11.2.2. Time course of the cardiovascular hemodynamics 144

11.2.3. The time course of the geometry of the left
ventricle

11.2.4. Transmural course of quantities related to
muscle mechanics

$\begin{array}{ll}\text { 11.2.5. Time course of coronary artery volume flow and } & \\ \text { transmural distribution of coronary perfusion } & 152\end{array}$

11.3. Simulations of deviant cardiac cycles 155

11.3.1. Introduction 155

11.3.2. Changes in end-diastolic volume and heart rate 156 
11.3.3. Simulation of changes in aortic input impedance

11.3.4. Coronary artery stenosis

11.3.5. Disturbed spread of activation across the myocardial wall

11.4. Validity of the model with respect to mathematical properties and assumptions

11.5. Sources of discrepancies of the model with the animal experiment

Chapter 12. SUMMARY AND CONCLUSIONS

Appendix 3.1. THE RELATION BETWEEN TANGENTIAL WALL TENSION AND INTERNAL PRESSURE IN A THIN-WALLED ROTATIONAL ELLIPSOID

Appendix 5. LEFT VENTRICULAR CAPACITANCE DURING SYSTOLE

A5.1. Capacitance of the left ventricle, consisting of isotropic material

A5.2. Capacitance of the left ventricle, consisting of anisotropic material

Appendix 6.1. COMPUTATION OF THE REFLECTION COEFFICIENT IN A BIFURCATION 185

Appendix 6.2. COMPUTATION OF THE CROSS-SECTIONAL STIFFNESS $K$

Appendix 6.3. COMPUTATION OF THE INTERNAL DIAMETER OF THE VESSEL SEGIIENT IN VIVO

SAMENVATTING

LEVENSBERICHT 
LIST OF SYMBOLS AND SUBSCRIPTS

Symbols

\section{Symbol Unit Meaning}

\begin{tabular}{|c|c|c|}
\hline A & - & degree of activation of muscle (relation 5.3.5) \\
\hline a & m & sarcomere length at zero load \\
\hline b & $m^{-1}$ & parameter passive muscle characteristics (relation 5.3.5) \\
\hline$b_{1}$ & $m^{-1}$ & parameter active muscle characteristics (relation 5.3.19) \\
\hline$c$ & $m^{5} \mathrm{~N}$ & capacitance $(\Delta V / \Delta p)$ \\
\hline d & $\mathrm{m}$ & diameter \\
\hline $\mathrm{F}$ & $\mathrm{N}$ & force \\
\hline $\mathrm{F}_{z}$ & N & axial force acting on upper surface cylinder \\
\hline f & - & coronary perfusion (relation 8.4.1) \\
\hline G & $\mathrm{N} \mathrm{m}^{-3}$ & force per sarcomere volume (relation 5.3.1) \\
\hline $\mathrm{G}_{\mathrm{aO}}$ & $\mathrm{N} \mathrm{m}^{-3}$ & parameter active muscle characteristics (relation 5.3 .8 ) \\
\hline$G_{p o}$ & $\mathrm{~N} \mathrm{~m}^{-3}$ & parameter passive muscle characteristics (relation 5.3 .5 ) \\
\hline H & $\mathrm{m}-2$ & initial height of the cylinder \\
\hline H & $\mathrm{N} \mathrm{m}^{-2}$ & modulus of elasticity (section 5.7 onty) \\
\hline h & $\mathrm{m}$ & height of the cylinder \\
\hline j & - & ordinal number of a subcylinder \\
\hline k & $\mathrm{N} \mathrm{m}^{-2}$ & cross-sectional stiffness (relation 6.2.1) \\
\hline L & $\mathrm{N} \mathrm{m}^{-5} \mathrm{~s}^{2}$ & flow inertance $[\Delta p / \Delta(d q / d t)]$ \\
\hline L & $\mathrm{m}$ & initial length (chapter 5) \\
\hline 1 & m & length \\
\hline $1_{s}$ & $\mathrm{~m}$ & sarcomere length \\
\hline $1_{\mathrm{sa}}$ & $\mathrm{m}$ & internal sarcomere length (characteristic quantity) \\
\hline $1_{\text {so }}$ & $\mathrm{m}$ & initial sarcomere length \\
\hline lss & m & length hypothetical series elastic element \\
\hline 1 sl & $\mathrm{m}$ & parameter active muscle characteristics (relation 5.3.20) \\
\hline $\mathrm{N}$ & - & number of distal branches of $0.4 \mathrm{~mm} \mathrm{I.D.} \mathrm{(section} \mathrm{6.2)}$ \\
\hline $\mathrm{n}$ & - & number of subcylinders \\
\hline $\mathrm{p}$ & $\mathrm{N} \mathrm{m}^{-2}$ & pressure \\
\hline$p_{\text {im }}$ & $\mathrm{N} \mathrm{m}^{-2}$ & intramyocardial pressure (section 5.6 ) \\
\hline$q$ & $m^{3} s^{-1}$ & volume flow \\
\hline$R$ & m & initial radius \\
\hline R & $\mathrm{N} \mathrm{m}^{-5} \mathrm{~s}$ & flow resistance \\
\hline
\end{tabular}




\section{Symbol Unit Meaning}

\begin{tabular}{|c|c|c|}
\hline$r$ & m & radius \\
\hline$r_{j}$ & m & inner radius \\
\hline$r_{0}$ & $\mathrm{~m}$ & outer radius \\
\hline$r_{i v}$ & $\mathrm{~m}$ & inner radius thick-walled cylinder (ventricle) \\
\hline$r_{\text {ov }}$ & m & outer radius thick-walled cylinder (ventricle) \\
\hline$S_{p}$ & $\mathrm{~N} \mathrm{~m}^{-2}$ & parameter microcirculation (relation 6.3.2) \\
\hline$s_{r}$ & $\mathrm{Nm} \mathrm{s}$ & parameter microcirculation (relation 6.3.3) \\
\hline $\mathrm{s}_{\mathrm{c}}$ & - & parameter microcirculation (relation 6.3.2) \\
\hline$T_{z}$ & $\mathrm{~N} m$ & axial torque upper surface cylinder \\
\hline $\mathrm{t}$ & s & time \\
\hline$t_{\text {cycl }}$ & s & duration cardiac cycle \\
\hline$t_{s}$ & $\mathrm{~s}^{3}$ & time related to process of sarcomere contraction \\
\hline$v^{3}$ & $\mathrm{~m}^{3}$ & volume \\
\hline$v_{e j}$ & $\mathrm{~m}^{3}$ & stroke volume \\
\hline$v^{e j}$ & $\mathrm{~m} \mathrm{~s}^{-1}$ & velocity of sarcomere shortening \\
\hline$v_{\mathrm{ao}}$ & $\mathrm{ms} \mathrm{s}^{-1}$ & parameter active muscle characteristics (relation 5.3.8) \\
\hline$v_{s l}$ & $\mathrm{~ms} \mathrm{~s}^{-1}$ & parameter active muscle characteristics (relation 5.3.20) \\
\hline$W$ & $\mathrm{Nm}$ & work, energy \\
\hline w & $\mathrm{N} \mathrm{m}^{-2}$ & specific energy $=$ energy/volume (section 8.3 ) \\
\hline$y$ & $x \times$ & characteristic quantity (chapter 4 ) \\
\hline Z & $\mathrm{N} \mathrm{m}^{-5} \mathrm{~s}$ & flow impedance \\
\hline$z_{\text {ao }}$ & $\mathrm{N} \mathrm{m}^{-5} \mathrm{~s}$ & characteristic impedance of the aorta \\
\hline$\alpha$ & $\mathrm{rad}$ & torsion angle (section 7.1 ) \\
\hline B & $\mathrm{rad}$ & fiber angle (subsection 3.3 .2 ) \\
\hline$B_{0}$ & $\mathrm{rad}$ & initial fiber angle \\
\hline$\gamma$ & $\mathrm{rad}$ & shear angle (relation 7.3 .4 ) \\
\hline$\varepsilon$ & - & natural strain (In [ $\mathrm{l} / \mathrm{L}]$ \\
\hline$\lambda$ & - & leakage fraction (section 6.2 ) \\
\hline$\eta$ & $\mathrm{N} \mathrm{m}^{-2} \mathrm{~s}$ & viscosity of blood \\
\hline$\rho$ & $\mathrm{kg} \mathrm{m}^{-3}$ & density of blood \\
\hline p & $\mathrm{m}$ & radius of curvature \\
\hline 0 & $\mathrm{~N} \mathrm{~m}^{-2}$ & stress \\
\hline$\tau$ & s & delay of relaxation of muscle (relation 5.3.18) \\
\hline$\tau_{s 1}$ & s & parameter active muscle characteristics (relation 5.3.18) \\
\hline$\tau_{s 2}$ & $s^{-1}$ & $\begin{array}{l}\text { parameter active muscle characteristics (relation 5.3.18) } \\
\text { angular frequency }\end{array}$ \\
\hline
\end{tabular}


Subscripts

\begin{tabular}{|c|c|}
\hline Subscript & Meaning \\
\hline a & active \\
\hline ao & aorta \\
\hline $\operatorname{art}$ & arterial, belonging to aortic input impedance \\
\hline $\mathrm{b}$ & coronary artery branch \\
\hline c & coronary \\
\hline ce & large coronary arteries (I.D. $>400 \mu \mathrm{m}$ ) \\
\hline $\mathrm{ca}$ & coronary arterioles \\
\hline $\mathrm{cc}$ & coronary capillaries \\
\hline$c v$ & coronary venules \\
\hline e & entrance of a vessel (segment) \\
\hline$f$ & muscle fibers \\
\hline im & intramyocardial \\
\hline j & ordinal number of subcylinder \\
\hline $1 v$ & left ventricle \\
\hline m & muscle \\
\hline$p$ & passive \\
\hline$r$ & radial direction \\
\hline ra & right atrium \\
\hline s & sarcomere \\
\hline$t$ & tangential direction \\
\hline $\operatorname{tr}$ & transmural \\
\hline $\operatorname{tra}$ & transmural arterioles \\
\hline $\operatorname{trc}$ & transmural capillaries \\
\hline $\operatorname{trv}$ & transmurâl venules \\
\hline vc & vasoconstrictive \\
\hline$z$ & axial direction \\
\hline $1,2,3$ & principle directions \\
\hline
\end{tabular}


1. GENERAL INTRODUCTION

In this thesis a mathematical model of the dynamics of the left ventricle and its coronary circulation is presented. The original aim of the present study was to gain more insight in the coronary circulation. It is known that in case of insufficient coronary flow, critical flow levels first are reached in subendocardial layers of the left ventricle ${ }^{9,22)}$. For this reason, special attention is paid to transmural differences in the coronary perfusion. Local coronary perfusion depends on several factors, such as metabolic needs, aortic pressure, local resistance of the coronary vasculature and the tine passed after the onset of the cardiac contraction. The resistance of the coronary vasculature changes during the cardiac cycle since the stresses in the ventricular wall acting during systole compress the coronary vasculature. This squeezing effect is particularly pronounced in the subendocardial layers of the left ventricle, where it causes arteriolar backflow and increased venular forward flow during systole 25$)$. Compression of the coronary circulation during systole is most proninent in the left ventricular wall because the stresses are highest here. Blood flow to the ventricles mainly occurs in diastole, when the stresses are minimum. In systole perfusion of the left ventricular wall is likely to be confined to the subepicardial layers.

In animal experiments determination of wall stress and its influence on the coronary circulation is rather inaccurate and incomplete. To get better insight in these and other relations the dynamics of the left ventricle and its coronary circulation are simplified to a model in which the most relevant quantities are incorporated. Probably, this model allows a more accurate calculation of the wall stress. In the present study a mathematical model is preferred because of the flexibility in varying its parameters.

In general, a mathematical model consists of a set of mathematical relations between relevant quantities. A number of these relations may contain parameters which govern the mutual influence of quantities. One of the most important factors of influence on the validity of a model is the reliability of the values of its parameters. Parameters can be determined either directly or by estimation. Direct determination of parameters can be done by experiment or by applying physical principles. Estimation of parameters is based on variation of the value of those parameters until the results of the model fit experimental data best. As a rule direct determination of the values of the parameters rather than estimation is to be preferred, since by direct determination any 
occurring error is localized to a smaller part of the model. As a consequence tracing of errors is facilitated. Moreover, a large number of estimated parameters results in an unacceptable unreliability of the model. In this situation, afterall, a good fit of the model to the experiments can be found although the model is defective because of inaccuracy of the individual relations. In conclusion, the best strategy is to deternine directly the values of as much of the parameters as possible, and to estimate the remaining unknown parameters.

A model in which the dynamic behavior of the wall stresses and their effect on the coronary circulation are analysed could not be found in literature. Only a few models of the dynamic behavior of the coronary circulation have been described $5,7,12)$. Although special reference has been made to transmural differences in coronary perfusion, these models are less suitable to study the effect of wall stresses on the coronary circulation because a thorough description of intramyocardial pressure is missing.

Several models of the mechanics of the left ventricle are reported in literature. They can be classified in models describing the geometry and models describing stresses in the ventricular wall. Models concerning the geometry of the heart are commonly used to predict ventricular geometry from a few dimensions, estimated angiographically or echocardiographically. To study the influences of cardiac contraction on the coronary circulation analysis of stresses in the wall of the ventricle is more relevant. Though several investigators are rather optimistic $3,6,16)$, direct determination of these stresses in animal experiments is difficult and unreliable ${ }^{13}$ ). A better approach is to calculate these stresses from the left ventricular pressure, assuming a certain geometry of the ventricle. Hanna ${ }^{10)}$ and Mirsky ${ }^{17)}$ proposed a spherical geometry of the left ventricle. A more accurate description of the left ventricle is a prolate ellipsoid as proposed by Hood et al. ${ }^{11}$, Mirsky ${ }^{18)}$, Streeter et al. 24) and Wong et al. 27). The finite element method applied to a realistic, but more complicated geometry of the heart was used by Janz 14,15 ) and Pao et ar. 19,20).

McHale et a ${ }^{16)}$ and recently Back ${ }^{1}$ ) showed that the magnitude of the calculated wall stress depends on the presumed geonetry of the heart. From these studies it can be concluded, that a presumed spherical geometry results in a value of the calculated wall stress, which is approximately 50\% lower than that value, as calculated on the base of a more realistic, elipsoidal geometry. In chapter 3 this difference will be discussed. Another problem is that the calculated stress distribution across the myocardial wall depends, among other 
things, on the mechanical properties of the myocardial material, as applied in the model. Many authors $8,11,14,15,17,18,19,20,27)$ assumed an isotropic behavior of the myocardial material. The myocardium, however, consists mainly of anisotropic muscle material. As will be shown in the chapters 5 and 11 of this thesis, models based on isotropic myocardial material properties introduce large errors, in the description of the mechanical behavior of the ventricle, especially with respect to the process of ejection and the transmural distribution of stresses. During ejection the simulated, isotropic ventricle appears to be three times stiffer than the real ventricle. Hence, models based on isotropic material properties of the heart cannot adequately represent the mechanics of the heart.

Another group of investigators accounted for the physiological contractile behavior of cardiac muscle $2,4,21,26)$ as derived from experiments on isolated papillary muscle. However, these models are based on a thin-walled geometry and do not consider transmural differences and changing fiber orientation.

The models presented in literature cannot be used to study the dynamic behavior of the wall stresses and their effect on the coronary circulation, since these models are based on unacceptable approximations such as isotropic cardiac material, inadequate geometry, no distinction of fiber orientation, or no physiological contractile behavior.

To design a model in which the wall stresses and their influence on the coronary circulation can be studied, information about these stresses has to be obtained. Longitudinal stress in the muscle fibers contributes to ventricular pressure as a consequence of the curvature of the muscle fibers around the ventricular cavity. This phenomenon is based upon Laplace's law, which firstly was applied to the heart by Hood ${ }^{28)}$. Since the orientation of the fibers varies across the myocardial wal1 ${ }^{23}$ ), the contribution of different layers to the ventricular pressure is not the same. This results in a non-linear decrease to negative values of the radial stress $\sigma_{r}$ from the epicardium $\left(\sigma_{r}=\right.$ 0 ) to the endocardium ( $\sigma_{r}$ equals, but is opposite to the left ventricular pressure; stress is negative pressure). In the present thesis the intramyocardial pressure, the pressure in the myocardium, is assumed to be equal and opposite to the radial stress. This pressure appears to be an important factor in the degree of compression of the coronary vasculature, directly influencing the coronary resistance. In literature intramyocardial pressure is often defined as the pressure acting on the outside of the coronary vessels. This definition, however, is not precise since pressure in inhomogeneous ani- 
sotropic material, as the myocardium, cannot be defined accurately. In chapter 3 and in more detail in chapter 5 intramyocardial pressure will be discussed. As a consequence of the importance of the stress in the myocardium a considerable part of the relations in the present model deals with the mechanics of the heart.

The new model presented in this thesis considers the effect of

- transmural differences in stress, strain and coronary perfusion

- compression of the coronary vasculature due to cardiac contraction

- changing fiber orientation across the myocardial wall

- anisotropic behavior of cardiac muscle

- contractile behavior of cardiac muscle, as measured experimentally

- thick walled cylindrical geometry of the left ventricle

- physiological hemodynamic loading of the heart. 


\section{Literature}

1. Back, L.: Left ventricular wall and fluid dynamics of cardiac contraction. Mathematical Biosciences 36, 257 - 297 (1977).

2. Brutsaert, D.L., PauTus, W.J.: Loading and performance of the heart as muscle and pump. Cardiovasc Res 11, 1 - 16 (1977).

3. Burns, J.W., Cove11, J.W., Myers, R., Ross, J.: Comparison of directly measured left ventricular wall stress and stress calculated from geometric reference figures. Circ Res 28, 611 - 621 (1971).

4. Covell, J.W., Fuhrer, J.S., Boerth, R.C., Ross, J.: Production of isotonic contractions in the intact canine left ventricle. J Appl Physiol 27, $577-581$ (1969).

5. Downey, J.M., Downey, H.F., Kirk, E.S.: Effects of myocardial strains on coronary bloodflow. Circ Res 34, 286 - 292 (1974).

6. Feigl, E.0., Simon, G.A., Fry, D.L.: Auxotonic and isometric cardiac force transducers. J Appl Physiol, 23, 597 - 600 (1967).

7. Gordon, R.J.: A general mathematical model of coronary circulation. Am J Physiol 226, 608 - 615 (1974).

8. Gould, P., Ghista, D., Brombolich, L.: In vivo stresses in the human left ventricular wall: analysis accounting for the irregular 3-dimensional geometry and comparison with idealised geometry analyses.

$\mathrm{J}$ Biomech 5, 521 - 539 (1972).

9. Griggs, D.M., Tchokoev, V.V., Chin chi Chen: Transmural differences in ventricular tissue substrate levels due to coronary constriction. Am $J$ Physiol 222, $705-709$ (1972).

10. Hanna, W.T.: A simulation of human heart function. Biophys J 13, $603-$ 621 (1973).

11. Hood, W.P. Jr., Thomson, W.J., Rackley C.E., et al.: Comparison of calculations of left ventricular wall stress in man from thin-walled and thick-walled ellipsoidal models. Circ Res 24, 575 - 582 (1969).

12. Horikawa, M., Chino, M., Takahashi, 11., Nagoshi, H.: Studies on the coronary circulation by a simulation method. Jpn Heart J 18, $246-258$ (1977).

13. Huismans, R.M.: Forces in the wall of the left ventricle. Thesis Free University Amsterdam, the Netherlands (1977).

14. Janz, R.F., Grimm, A.F.: Finite-element model for the mechanical behaviour of the left ventricle. Circ Res 30, $224-252$ (1972).

15. Janz, R.F., Grimm, A.F.: Deformation of the diastolic left ventricles: I. Nonlinear elastic effects. Biophys J 13, $689-704$ (1973). 
16. Mchale, P.A. Greenfield, J.C.: Evaluation of several geometric models for estimation of left ventricular circumferential wall stress. Circ Res 33, $303-312(1973)$.

17. Mirsky, I.: Ventricular and arterial wall stresses based on large deformation analysis. Biophys J 13, 1141 - 1159 (1973).

18. Mirsky, I.: Left ventricular stresses in the intact human heart. Biophys J $9,189-208(1969)$.

19. Pao, Y.C., Ritman, E.L., Hood, E.H.: Finite element analysis of left ventricular myocardial stresses. J Bionech 7, $469-477$ (1974).

20. Pao, Y.C., Robb, R.A., Ritman, E.L.: Plain-strain finite-element analysis of reconstructed diastolic left ventricular cross-section. Ann Biomed Eng 4, 232 - 249 (1976).

21. Paulus, W.L., Claes, V.A., Brutseart, D.L.: Physiological loading of isolated mammalian cardiac muscle. Circ Res 39, $42-53$ (1976).

22. Schaper, W.K.A.: The collatoral circulation of the heart. North Holland, Amsterdanı (1971).

23. Streeter, D.D. Jr., Hanna, H.T.: Engineering mechanics for successive states in canine left ventricular myocardium. II. Fiberangle and sarcomere length. Circ Res 33, 656 - 664 (1973).

24. Streeter, D.D., Vaishnav, R.N., Patel, D.J., Spotnitz H.M., Ross, J., Sonnenbiick, E.H.: Stress distribution in the canine left ventricle during diastole and systole. Biophys J 10, 345 - 363 (1970).

25. Tillmans, H., Ikada, S., Hansen, H., Jonnalagedda, S., Sarma, M., Fauvel, J.M., Bing, R.: Microcirculation in the ventricle of the dog and turtle. Circ Res 34, 561 - 569 (1974).

26. Weber, K.T., Janicki, S.J., Hefner, L.L.: Left ventricular force-length relations of isovolumic and ejecting contraction. Am J Physiol 231, $337-343(1976)$.

27. Wong, A.Y.K., Rautoharju, P.M. : Stress distribution within the left ventricular wall approximated as a thick ellipsoidal shell. Am Heart J 75, $649-661$ (1968).

28. Woods, R.H.: A few applications of a physical theorem to membranes in the human body in a state of tension. J Anat Physiol 26, 362 - 370 (1892). 
2. BASIC ANATOMY AND PHYSIOLOGY OF THE HEART AND THE CORONARY ARTERIES

The anatomy and physiology of the heart and coronary circulation are rather complicated. This chapter confines itself to a brief review of some anatomical and physiological aspects of these structures. The present information, derived from textbooks $1,2,3$ ), is basic and especially meant for readers who are not familiar with anatomy and physiology of the heart. More detailed information is given in the specific chapters.

Fig. 2.1 shows diagramatically a sagital cross-section of the heart. The heart consists of four cavities, the right (RA) and left (LA) atria and the right (RV) and left (LV) ventricles. The veins, which collect the blood from the systemic circulation, empty into the right atrium, whereas the pulmonary veins empty into the left atrium. The right atrium is separated from the right ventricle by the tricuspid valve (TV), whereas the left atrium is separated from the left ventricle by the mitral valve (MV). The pulmonary valve (PV) is situated between the right ventricle and the pulmonary trunk (PT). The puimonary trunk bifurcates after a short distance in the left and right pulmonary arteries. The aortic valve (AV) separates the left ventricle from the aorta $(A 0)$. In the dog the first large side branches of the aorta are the brachio cephalic artery and the left subclavian artery, which supply the head and the upper extremities with blood. In humans three branches of the aorta supply these structures with blood. The ventricular septum is the wall which separates both ventricles. The other walls of the ventricles are the free walls. A number of papillary muscles (PPM) originate from the ventricular walls and are connected by the chordae tendinae to the leaflets of the tricuspid and mitral valve, respectively. The valves of the heart are situated in the basal plane. Across the ventricles, opposite to the basal plane, is the apex of the heart. At this site, the ventricular walls are relatively thin.

In fig. 2.2 a cross-sectional view of the ventricular part of the heart is shown diagramatically. The plane of cross-section is indicated in fig. 2.1 by the dot and dash line AA. The free wall of the right ventricle is significantly thinner than that of the left ventricle because of a lower pressure in the right than in the left ventricle. The ventricular septum is slightly thinner than the free wall of the left ventricle. The cross-section of the left ventricle is virtually circular. The inner surface of a ventricle, the endocardium, is irregular. The outer surface of the heart is the epicardium. The myocardium is the muscular tissue between the endo- and epicardium. The subendo- 


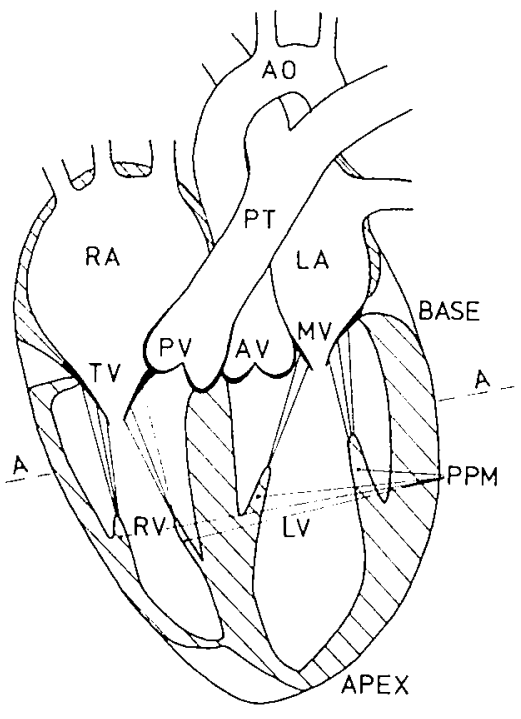

Figure 2.1.

Section of the heart in the sagittal plane. $R A=$ right atrium, $L A=1 \mathrm{eft}$ atrium, $R V=$ right ventricle, $L V=$ left ventricle, TV = tricuspid valve, $M V=$ mitral valve, $P V=$ pulmonary valve, $A V=$ aortic valve, $P T=$ pulmonary trunk, $A O=$ aorta, $P P M=$ papillary muscles.

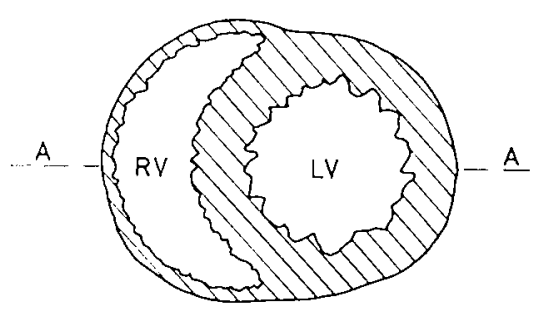

Figure 2.2.

Cross-section of the ventricle in a plane which is indicated as $A A$ in fig. 2.1 .

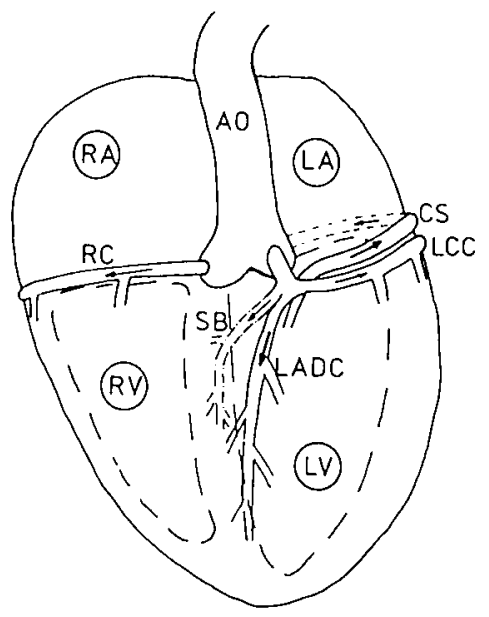

Figure 2.3.

The epicardial coronary vessels. $\mathrm{RC}=$ right coronary artery, $L C C=$ circumflex branch of the left coronary artery, $L A D C=$ anterior descending branch of the left coronary artery, $S B=$ septal branch, $\mathrm{CS}=$ coronary sinus. 
cardium and the subepicardium are the inner and superficial layers of the myocardium, respectively.

The coronary arteries (fig. 2.3) originate from the aorta, just distal to the aortic valves. They run over the heart, partly embedded in the subepicardium. The right coronary artery (RC) predominantly perfuses the right ventricular free wall and occasionally part of the posterior side of the left ventricular free wall and septum. In dogs the left coronary artery is usually short. Its first main side branch is the septal branch (SB) which perfuses the septum and an important part of the conductive system. Just distal to the septal branch the left coronary artery bifurcates in a circumflex branch (LCC) and an anterior descending branch (LADC). The LCC runs along the basal plane and perfuses a major part of the anterior, marginal and posterior side of the left ventricular free wall. The LADC runs towards the apex following the borderline between the left and right ventricle and perfuses part of the anterior side of the left and right ventricular free walls and part of the septum. In humans the left coronary artery is usually longer than in the dog and a distinct septal branch is absent. In this species the septum and the conductive system in this structure are commonly supplied with blood through small side branches of the left coronary artery and LADC. The contribution of the LADC in the blood supply of the anterior part of the ventricular free wall is generaliy larger in humans than in dogs. The coronary sinus (CS) is the largest coronary vein and collects most of the blood from the heart. It runs parallel to the LCC in the same direction, and empties into the right atrium at the posterior side of the heart.

During the cardiac cycle the following events occur. Late in systole and in diastole, which is defined as the non-active period, blood flows into both atria. This results in an increase in pressure in the atria. Because of this pressure increase and the relaxation of the ventricles in this phase of the cardiac cycle, the mitral and tricuspid valves open, and the ventricles are filled. The filling of the ventricles is completed by the contraction of the atria initiated by depolarisation of the sinus node. Then the ventricles contract and the systolic phase starts. The tricuspid and mitra? valves close as a result of the increase in pressure in the ventricular cavities. In this phase of the cardiac cycle volume remains constant because all valves are closed. Therefore, this phase is called the isovolumic contraction phase. Next the pulmonary and aortic valves open because the right and left ventricular pressures exceed the pressures in the pulmonary trunk and aorta, re- 
spectively. The ejection phase has started. A fast rise in ventricular outflow is followed by a slower decline in flow. As a result of the relaxation of the heart the outflow stops and the puimonary and aortic valves close. The ventricular volumes are constant again while ventricular pressures decrease. This phase is named the isovolumic relaxation phase. As a consequence of complete relaxation the atria are filled and diastole starts again. 


\section{Literature}

1. MCAlpine, W.A.: Heart and coronary arteries. Springer Verlag, Berlin (1975).

2. Miller, M.E., Christensen, G.C., Evans, H.E.: Anatomy of the dog. H.B. Saunders Company, Philadelphia (1964).

3. Mountcastle, V.B.: Medical physiology II. 13th ed. The C.V. Mosby Company, Saint Louis (1974). 


\section{DESIGN OF A MATHEMATICAL MODEL OF THE LEFT VENTRICLE}

\subsection{Introduction}

The purpose and concise characteristics of the model of the left ventricle are described in chapter 1 . In chapter 2 the anatomy and function of the heart are shown to be of a complex nature. Accounting for all these properties would result in a model with so many parameters, that insight in the system becomes difficult. Moreover, the introduction of too many parameters readily can suggest agreement of the data obtained from the model and those obtained from animal experiments, whereas the physical or physiological principles of the model may be incorrect. Therefore, simplifications concerning the characteristics of the heart and its hemodynamics are required. Each simplification as proposed is a compromise between accuracy and simplicity in the description of the biological situation. Following this principle, the following simplifications are made:

- only the left ventricle is simulated

- the left ventricle is rotationally symmetric in all properties

- the left ventricle is a thick-walled cylinder, composed of a number of fitting concentric subcylinders (fig. 3.1)

- the myocardial tissue consists of muscle material, which is simulated by a contractile fiber structure embedded in a soft, incompressible material

- the contractile properties of the muscle fibers are described by a stressstrain-strainrate-time relation, as described in chapter 5

- the fiber orientation changes across the myocardium, all fibers being locally paralle?

- the endocardium and chordae tendinae can bear forces only in the axial direction

- the sequence of the onset of muscle contraction is merely radial

- the aortic valve has no resistance in case of forward flow; backflow is zero, when there is a backward pressure gradient

- the aortic input impedance is simulated by a resistance in series with a capacitance, which is in parallel with a second resistance (fig. 3.2)

- the left ventricular filling rate is zero during the first $50 \%$ of the cardiac cycle, starting with systole, and is constant during the last $50 \%$ of the cardiac cycle

- the volume of the intramyocardial coronary circulation, which changes during the cardiac cycle, is added to the volume of the myocardium in such a way 

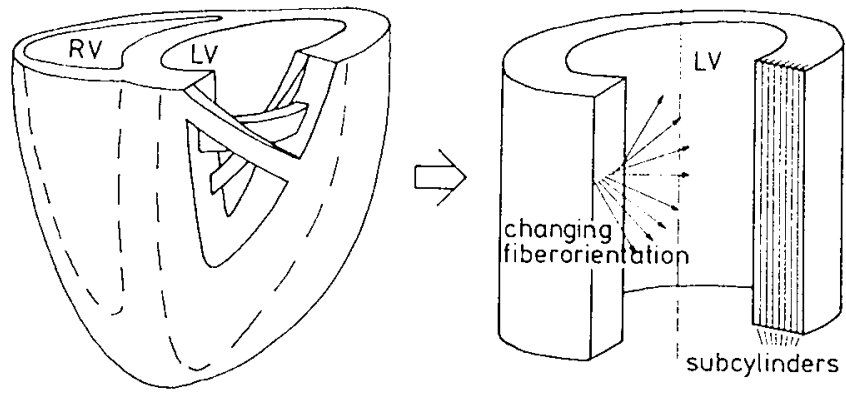

Figure 3.1 .

Schenatic representation of the geometry of the left ventricle. The ventricle is simulated by a cylinder. Subdivision of the thick walled cylinder in a number of subcylinders and changing fiber orientation are indicated.

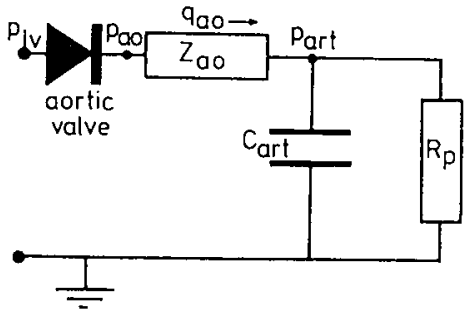

Figure 3.2.

Network which represents the aortic input inpedance including the aortic valve.

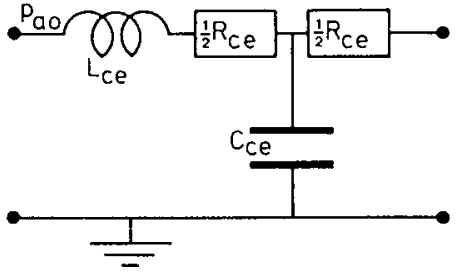

Figure 3.3.

Network which represents the large coronary arteries (I.D. > $400 \mu \mathrm{m}$ ). The left side represents the proximal side of these arteries. 
that the sarcomere length is not affected and the density of muscle fibers per cross-sectional area varies

- the large coronary arteries (I.D. > $400 \mu \mathrm{m}$ ) are simulated by a four element mode $i$, composed of two resistances, one capacitance and one inertance (fig. 3.3)

- the coronary arterioles, capillaries and venules possess resistive and capacitive characteristics only

- the resistance of the arterioles, capillaries, and venules depends on their respective volume according to Poisseuille's law

- the external pressure acting on the coronary vessels equals the hydrostatic pressure in the hypothetical soft, inconpressible material surrounding the muscle fibers, and is defined as the intramyocardial pressure

- the vasoactivity of the arterioles is simulated by a vasoconstrictive pressure, which is superimposed on the external pressure acting on the coronary blood vessels.

The simplifications are discussed below in more detail.

\subsection{Simplifications concerning the geometry of the heart}

\subsubsection{Consideration of the left ventricle}

In the compromise between the loss in completeness and the gain in simplicity the right ventricle is not incorporated in the model. The gain in simplicity is evident, since several not well known parameters, describing the geometry and the hemodynanic loading of the right ventricle can be omitted. The loss in accuracy is limited since under normal conditions the right ventricle contributes to approximately $20 \%$ of the hemodynamic power, supplied by the heart. Moreover, the filling and contraction characteristics of the left ventricle are hardly influenced by the degree of filling of the right ventricle in the normal range of end-diastolic volumes $5,11,19)$. Another justification of the exclusion of the right ventricle is that in case of insufficient coronary flow critical perfusion levels are at first reached in the subendocardial layers of the left ventricle 7,20$)$. During systole the left ventricular subendocardial tissue pressure, acting on the outside of the coronary vasculature, is of the same order of magnitude as the coronary perfusion pressure. Thus in that region the coronary circulation is strongly influenced by the mechanical contraction of the heart. 


\subsubsection{Rotational_symetry of the left ventricle}

Leaving the right ventricle out of consideration, the left ventricle has no asymmetric external geometry. The internal geometry is approximately rotationally symmetric (fig. 2.3), when the papillary muscles are ignored. Therefore, many authors assume a rotational symmetric geometry of the left ventricle, such as a sphere $3,8,13,15)$, an ellipsoid $3,6,13,14,18,26,29)$, a cylinder ${ }^{1,13)}$ or an arbitrary rotational symmetric geometry ${ }^{10,17)}$. Since tissue properties probably do not vary along the circumference of the left ventricle, the left ventricle is described sufficiently accurate by rotational symmetric geometry and properties. As a result of this simplification the tangential coordinate is not relevant.

\subsubsection{The cylindrical geometry of the left ventricle}

As mentioned in section 3.2 .2 several hypothetical rotationally symnetric shapes of the left ventricle have been described in the literature. An important test of the validity of the geometry of the simulated left ventricle is the relation between internal cavity pressure $(p)$ and the tangential component of the wall tension. In deriving this relation both a sphere and a cylinder are considered as limiting cases of an ellipsoid. In this concept calculations are based on a thin-walled geometry. A thin-walled prolate ellipsoid is characterised by two parameters, the minor axis $d_{1}$ and the major axis $d_{2}$ (fig. 3.4). The tangential component $\left(T_{1}\right)$ of the wall tension at the equator (0 in fig. 3.4) will be related to the internal pressure (p).

Figure 3.4.

Application of Laplace's law to the left ventricular wall, which is represented by a prolate ellipsoid.

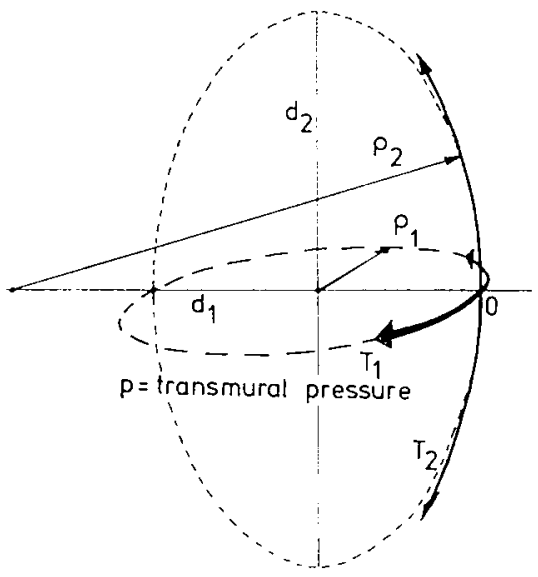


The external pressure is assumed to be zero. According to Laplace's law the following relation in point 0 holds:

$$
p=\frac{T_{1}}{P_{1}}+\frac{T_{2}}{P_{2}}
$$

where $T_{2}=$ the axial component of the wall tension in $0\left(\mathrm{~N} \mathrm{~m}^{-1}\right)$

$\rho_{1}=$ the tangential radius of curvature in $0(\mathrm{~m})$

$\rho_{2}=$ the axial radius of curvature in $0 \quad(m)$

Since the ellipsoid is rotationally symmetric, the axial component of the wall tension $T_{2}$ can be eliminated from relation (3.2.1), as shown in appendix 3.1. Thus it follows:

$$
T_{1}=\left(1-\frac{\rho_{1}}{2 \rho_{2}}\right) p_{\rho_{1}}
$$

Bove et al. ${ }^{1)}$ and Sandler et al. ${ }^{18)}$ demonstrated in their experiments, that the ratio of $\rho_{2}$ and $\rho_{1}$ ranges from 5 to 10 or even more. Substituting the minimum value 5 into relation $(3.2 .2)$ renders:

$$
p \rho_{1}>T_{1} \geq 0.9 p p_{1}
$$

Simulation of the left ventricle by a sphere implies $\rho_{1}=\rho_{2}$, resulting in

$$
T_{1}=0.5 \mathrm{p} \rho_{1}
$$

Simulation of the left ventricle by a cylinder implies $\rho_{2}+\infty$, resulting in

$$
\mathrm{T}_{1}=1.0 \mathrm{p} \rho_{1}
$$

The relation (3.2.2) valid for the ellipsoid looks to be the best one since this geometry is a good approximation of the actual left ventricle. Calculations on the base of an ellipsoidal geometry, however, are complicated. Therefore, simplifications are required, and comparison is made with a spherical and cylindrical geometry. From the relations (3.2.3) and (3.2.4) it follows that a simulation based on a sphere underestimates the tangential wall tension by $44 \%$ to $50 \%$, as compared to the more realistic ellipsoidal geometry. 
From the relations (3.2.3) and (3.2.5) it follows that a simulation based on a cylinder overestimates the tangential wall tension by maximally $11 \%$. These characteristic differences between the different geometries are confirmed by literature $1,3,13)$. In the present model the left ventricle is simulated by a cylinder, since in that case the error in the wall tension is moderate compared to simulation by a sphere. The description by a cylinder is much simpler than by an ellipsoid as a consequence of the irrelevancy of the axial coordinate. For the latter reason no attempt is made to base the rotationally symmetric geometry on the actual silhouette of the left ventricle. Another consideration, supporting the use of a cylindrical geometry, is that the majority of the myocardial tissue is present in the equatorial region of the left ventricle, where the myocardium is very similar to a cylinder ${ }^{24)}$. Obviously, close to the apex, the geometry of the left ventricle cannot be approximated by a cylinder, since there the axial and tangential radii of curvature of the ventricular wall are in the same order of magnitude.

The left ventricle cannot taken to be a thin-walled cylinder. A normal enddiastolic value of the ratio of wall thickness to internal radius is in the order of 0.5 (chapter 9), and increases considerably during contraction. The simplest method for computing the mechanical stresses is subdivision of the wall of the left ventricle in a sufficient number of fitting concentric subcylinders (chapter 7), thus involving a thin-walled behavior of the separate subcylinders.

\subsection{Simplifications concerning the properties of the myocardial wall}

\subsubsection{The mechanical properties of the myocardial tissue}

For an adequate model of the dynamics of the left ventricle the relation between local stress and local strain is necessary. Both stress and strain are defined by 3 normal and 3 shear components. The relation between the tensile stress (= muscle force/cross-sectional area) and the corresponding natura? strain (= the logarithm of muscle length/reference muscle length) are derived from experimental studies on myocardial material (chapter 5). In the model visco-elastic properties of the myocardium during diastole are neglected. During activation of the myocardium stress depends on velocity of shortening. Yet, two additional relations are necessary to describe the relation between stress and strain completely. Two different concepts of material properties, both resulting in the necessary additional relations, are evaluated: 
1) the muscle material is isotropic and pre-strained in the direction of the muscle fibers;

2) the muscle material is anisotropic, consisting of a contractile muscle fiber structure embedded in a soft, incompressible material.

In chapter 5 these concepts are described and discussed in detail. The conclusion is, that the concept of anisotropic myocardial material is to be preferred.

\subsubsection{The fiber orientation in the myocardial wall}

The fiber orientation in the myocardium varies across the myocardial wal ${ }^{9}$, 27). Since the cylinder model simulates the equatorial region of the left ventricle in the best way, in the model the fiber orientation in the equatorial region of the left ventricular free wall is considered to be representative of the situation in the whole left ventricle. In the model all muscle fibers in each subcylinder are assumed to be in parallel with the endocardium and to intersect a plane perpendicular to the axis of the cylinder at the same angle. The value of this angle in each subcylinder is derived from the experiments of Hort $^{9)}$ and Streeter et al. 27).

In the model the muscle fibers are connected with the upper and lower crosssectional surfaces of the cylinder. In these surfaces forces as well as torques are in equilibrium. Forces, acting on such a surface, as a result of axial stresses in the cylinder, are counterbalanced by the force, acting on that surface as a result of the left ventricular cavity pressure. The equilibrium of torque on such surface is based on counteracting shear stresses, which tend to rotate the surface. In the real left ventricle the cylindrical shaped equatorial region of the left ventricle is confined by the apical region on one side and the basal region on the other side. Especially in the apical region the assumption of cylindrical geometry does not hold. However, the equilibria of forces and torques hold in the cross-sectional planes, separating the more cylindrical part from the end regions. Therefore, the model is a good representative of the equatorial region of the left ventricle, as far as the mechanical behavior is concerned.

\subsubsection{The mechanical properties of the endocardium}

The model is partiy based on equilibrium of forces (chapter 7). The data on the fiber orientation suggest that the myocardium itself is not in mechanical equilibrium (chapter 10). Especially in diastole the imbalance in axially di- 
rected forces is substantial. The experimental data on the base to apex motion during contraction agree (chapter 11) with the data generated by the present model, if an elastic endocardium is assumed, which is able to bear axial forces. This hypothesis may be supported by the fact that the endocardium contains a structure of fibrous tissue which is predominantly axially directed $^{12)}$. Apart from this the valves are connected to the papillary muscle through chords of fibrous tissue (the chordea tendinae), which can bear axial forces between apex and base. During diastole the endocardium and the chordea tendinae probably influence the shape of the left ventricle considerably since diastolic muscle stresses are low. The conclusion is that in the model an endocardial subcylinder, which is only able to bear forces in the axial direction, can represent sufficiently accurately the mechanical influences of all fibrous structures in the left ventricular cavity on myocardial performance.

\subsubsection{The seguence of the onset of contraction in the myocardium}

The onset of contraction of muscle material is preceeded by electrical depolarization of the muscle cells. Assuming that the time lag between the electrical depolarization and the onset of contraction is constant, the contraction sequence corresponds to the sequence of electrical depolarization. In the left ventricle electrical depolarization starts in the Purkinje system. The apical subendocardium is activated, and next the activation reaches the subendocardium at the base within $20 \mathrm{~ms}$. From the subendocardial layers the activation is spread to the subepicardium, where activation starts at the apex about 20 ms after onset of the activation of the subendocardial layers $3,21,22,23$ ) (fig. 3.5). The subepicardial layers at the base are activated approximately $50 \mathrm{~ms}$ after the onset of activation. The direction of the wave front of the depolarization is predoninantly radial. In the model, therefore, axial time lag in the depolarization is neglected, but the radial time lag is retained.

\subsection{The hemodynamic loading of the left ventricle}

\subsubsection{The aortic valve}

In the biological situation the aortic valve opens when systolic left ventricular pressure exceeds systolic aortic pressure. Because of inertia of the blood, the ventricular outflow increases as a result of a pressure drop from the ventricular cavity in the aortic direction. After the ventricular outflow has reached a maximum, the blood pressure in the ventricle is slightly lower 
than in the aorta and flow decelerates but is still forward because of the inertia of the blood. Due to the backward pressure gradient over the aortic valve, this valve starts closing as early as the end-systolic deceleration phase 24,25$)$. The flow through the valve opening decreases further until the direction of flow reverses. Next a minor backflow completes the closure of the aortic valve. Under physiological circumstances, however, the pressure difference over the aortic valve opening during forward flow, seldom exceeds $700 \mathrm{~N}$ $\mathrm{m}^{-2}$ ( $\left.=5 \mathrm{mmHg}\right)$. Horeover, the duration and magnitude of backflow under non-pathological circumstances is of little importance. Therefore, the aortic valve can be represented sufficiently accurate by an ideal element with the following characteristics: a zero pressure difference during forward flow and a zero flow when a backward pressure gradient is present.

\subsubsection{The aortic input impedance}

The aortic input impedance relates the flow through the aortic valve to the pressure just distal to that valve. This impedance is non-linear and depends on such factors as frequency, peripheral resistance and reflections in the arterial system. The non-linear effects can be bypassed by specifying a linear impedance for small pressure changes around the mean pressure at the orifice of the aorta. When accounting for physiological deviations from the mean aortic pressure no major mathematical difficulties are encountered as a result of non-linearities. Westerhof et al. 28) proposed a linear impedance consisting of a resistance $\left(Z_{\mathrm{ao}}\right)$ in series with a capacitance $\left(C_{\text {art }}\right)$ which is in parallel with another resistance $\left(R_{p}\right)$ (fig. 3.2). This linear three element model of the impedance approximates the actual impedance with an accuracy of better

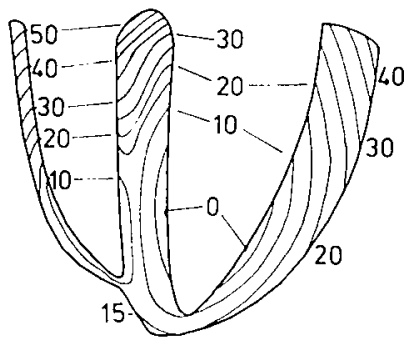

Figure 3.5 .

Sequence of electrical depolarization of the ventricular walls. The numbers indicate the delay time of the depolarization after the onset of ventricular depolarization in milliseconds.

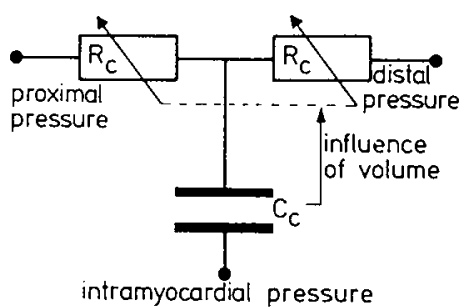

Figure 3.6 .

Network which represents a subcompartment of the coronary microcirculation. The resistance $R_{C}$ depends on the volume of the capacitance $C_{C}$ according to Poisseuille's law. 
then $15 \%$ between 0 and $10 \mathrm{~Hz}$ and better than $30 \%$ between 10 and $20 \mathrm{~Hz}$. However, large changes in mean aortic pressure have to be met by variation of the parameters characterising the impedance.

\subsubsection{The_left ventricular filling}

The left ventricle is filled through the mitral valve. In heal thy calves Nolan ${ }^{16)}$ found that the mitral flow normally had two maxima. The end-systolic phase is quickly followed by the first maximum. The second maximum is caused by atrial contraction. With increasing heart rate the maxima shift towards each other, which finally results in a fusion of both maxima. Since during diastole the stresses in the left ventricular wall are low and, therefore, of no particular importance for the coronary hemodynamics in general, the left ventricular inflow is simplified to no flow during the first 50\% of the cardiac cycle, starting with systole, and a constant value during the last 50\% of the cardiac cycle. The magnitude of the inflow is chosen in such a way that at the end of the heart cycle as well as at the onset of the contraction the left ventricular volume is the same.

\subsection{The coronary circulation}

\subsubsection{The volume of the intramyocardial coronary vessels}

The volume of the intramyocardial coronary vessels is a considerable part (approx. 12\%, chapter 9) of the total myocardial volume. During systole part of the venous coronary blood is squeezed from the myocardium into the coronary sinus, resulting in loss of mechanical energy from the myocardium. The model reveals that in the subendocardial layer approximately $6 \%$ of the mechanical energy which is generated by this layer, is dissipated by the squeezing effect. In the real left ventricle this ratio is not known. Therefore, in the model the volume of the coronary circulation is part of the myocardial volume. Volume changes of the coronary circulation are supposed to affect only the fiber density per cross-sectional area without affecting length and number of muscle fibers.

\subsubsection{The large coronary arteries (I.D. $>400$ um)}

The large coronary arteries distribute the blood over the surface of the myocardium, from which radially directed, smaller arteries enter the myocardium, locally supplying the myocardial tissue with blood. Because of the ge- 
onetry of the large coronary arteries the impedance of this arterial vasculature must be incorporated in the model (chapter 6). As a compromise between accuracy and simplicity the impedance is simulated by a four element model consisting of an inertance, a capacitance and two equal resistances (fig. 3.3 ).

\subsection{Vasoactivity}

Coronary flow is mainly regulated by the degree of constriction of the arterioles. This constriction is due to contraction of smooth muscle cells in the walls of the arterioles. The control of the vasoactivity mechanism depends in a complicated way on the metabolic balance of the myocardium. In the model only vasoactivity is simulated without considering the complete control system. The influence of the tension of the smooth muscle cells in the arteriolar wall is simulated by a vasoconstrictive pressure, which is superimposed to the 10cal intramyocardial pressure.

\subsubsection{The coronary arterioles, capillaries and venules}

Little is known about the dynamic behavior of the coronary arterioles, capillaries and venules, but some assumptions can be made. The inertance of coronary vessels, smaller than 1 mm in dianieter can be neglected, since in that case the related time constants are less than $10 \mathrm{~ms}$ (chapter 6 ). In each subcylinder, therefore, the arteriolar, capillary and venular vasculatures are simulated by networks, consisting of two equal resistances and a capacitance (fig. 3.6). The resistances depend on the volume of the corresponding vasculature according to Poisseuille's law. The volume of the coronary vessels is determined by the transmural pressure over the non-linearly elastic wall. Collapsing of coronary vessels is supposed to occur when somewhere in the coronary circulation the sum of the transmural pressure and the vasoconstrictive pressure becomes zero.

Estimates of the resistances are based on the static pressure measurements at various sites in the microcirculation. The external pressure acting on the vessels is defined as the intramyocardial pressure, and is supposed to be equal to the local pressure in the hypothetical soft, incompressible material surrounding the muscle fibers (section 5.6). The internal pressure of a coronary vessel equals the sum of the external pressure and the transmural pressure over the vessel wall. There is a unique relationship presumed between transmural pressure and internal volume. 
1. Back, L.: Left ventricular wall and fluid dynamics of cardiac contraction. Mathematical Biosciences 36, 257 - 297 (1977).

2. Bove, A.A., Lynch, P.R.: Radiographic determination of force-velocitylength relationshipsin the intact dog heart. J Appl Physiol 29, $884-888$ (1970).

3. Burns, J.W., Covel1, J.W., Myers, R., Ross, J.: Comparison of directly measured left ventricular wall stress and stress calculated from geometric reference figures. Circ Res 28, 611 - 621 (1971).

4. Durrer, D., van Dam, R.Th., Freud, G.E., Janse, M.J., Meyler, F.L., Arzbaecher, R.C.: Total exitation of the isolated human heart. Circulation 41. $899-912(1970)$.

5. Elzinga, G., van Grondelle, R., Westerhof, N., van den Bos, G.C.: Ventricular interference. Am J Physiol 226, $941-947$ (1974).

6. Ghista, D.N., Sandler, H.: An analytic elastic - viscoelastic model for the shape and the forces in the left ventricle. J Biomech 2, $35-47$ (1969).

7. Griggs, D.M., Tchokoev, V.V., Chin chi Chen: Transmural differences in ventricular tissue substrate levels due to coronary constriction. Am J Physiol 222, $705-709$ (1972).

8. Hanna, W.T.: A simulation of human heart function. Biophys J 13, $603-621$ (1973).

9. Hort, W.: Makroskopische und mikrometrische Untersuchungen am Myokard verschieden stark gefül lter linker Kammern. Virchows Arch (Pathol Anat) $333,523-564(1960)$.

10. Janz, R.F., Grimm, A.F.: Deformation of the diastclic left ventricles: I. Nonlinear elastic effects. Biophys J 13, $689-704$ (1973).

11. Langille, B.L., Jones, D.R.: Mechanical interaction between the ventricles during systole. Can J Physiol Pharmacol 55, 373 - 382 (1977).

12. McAlpine, W.A.: Heart and coronary arteries. Springer Verlag, Berlin (1975).

13. McHale, P.A., Greenfield, J.C.: Evaluation of several geometric models for estimation of left ventricular circumferential wall stress. Circ Res 33 , $303-312(1973)$.

14. Mirsky, I.: Left ventricular stresses in the intact human heart. Biophys $\mathrm{J}$ 9. $189-208$ (1969).

15. Mirsky, I., Parmley, W.W.: Assessment of passive elastic stiffness for isolated heart muscle and the intact heart. Circ Res 33, $233-243$ (1973).

16. Nolan, S.P.: The normal mitral valve: Patterns of instantaneous mitral valve flow and the atrial contribution to ventricular filling.

In: The mitral valve. Ed.: D. Kalmanson. Publ. Sciences Group Inc. Massachusetts, $137-143$ (1976). 
17. Pao, Y.C., Ritman, E.L., Wood, E.H.: Finite element analysis of left ventricular myocardial stresses. J Biomech 7, 469 - 477 (1974).

18. Sandler, H., Ghista, D.N.: Mechanical and dynamic implications of dimensional measurements of the left ventricle. Fed Proc 28, 1344 - 1350 (1969).

19. Santamore, W.P., Lynch, P.R., Meier, G., Heckman, J., Bove, A.A.: Hyocardial interaction between the ventricies. J Appl Physiol 41, $362-368$ (1976).

20. Schaper, W.K.A.: The collatoral circulation of the heart. North-Holland Pbl. Co., Amsterdam, Netherlands (1971).

21. Scher, A.H., Young, A.C., Malngren, A.L., et al.: Spread of electrical activity through the wall of the ventricle. Circ Res 1, $539-547$ (1953).

22. Scher, A.M., Young, A.C.: The pathway of ventricular depolarisation in the dog. Circ Res 4, $461-469$ (1956).

23. Sidney Harris, A.: The spread of axcitation in turtle, dog, cat and monkey ventricles. Ail J Physiol 134, $319-332$ (1941).

24. Spaan, J.A.E., van Steenhoven, A.A., van der Schaar, P.J., van Dongen, M. E.H., Smulders, P.T., Leliveld, H.H.: Hydrodynamical factors causing large mechanical tension peaks in leaflets of artificial triple. Trans Am Soc Artif Intern Organs 21, 396 - 402 (1975).

25. Van Steenhoven, A.A., van Dongen, M.E.H., Vaessen, E.C.J., Wasser, A.A.M.: Model experiments on the closing behavior of the natural aortic valve. Biomed Tech 22, 135 - 136 (1977).

26. Streeter, D.D. Jr., Hanna, H.T.: Engineering mechanics for successive states in canine left ventricular myocardium. I. Cavity and wall geometry. Circ Res 33, 639 - 655 (1973).

27. Streeter, D.D. Jr., Hanna, M.T.: Engineering mechanics for successive states in canine left ventricular myocardium. II. Fiberangle and sarcomere length. Circ Res 33, 656-664 (1973).

28. Westerhof, N., Enzinga, G., van den Bos, G.C.: Influence of central and peripheral changes on the hydraulic input impedance of the systematic arterial tree. Med Biol Eng, 710 - 722 (1973).

29. Wong, A.Y.K., Rautaharju, P.M.: Stress distribution within the left ventricular wall approximated as a thick ellipsoidal shell. Am Heart J $\underline{75}$, $649-661(1968)$. 
4. CHARACTERIZATION OF THE MODEL BY A SYSTEM OF DIFFERENTIAL EQUATIONS

\subsection{Introduction}

The model of the left ventricle, including its coronary circulation is a mathematical description of the biological situation. Commonly a mathematical model is a set of mathematical relations between quantities which are assumed to be predominantly relevant for the phenomena to be studied.

In chapter 1 the quantities which are considered to be relevant are mentioned. These are coronary volume flow, local myocardial tissue perfusion, 10cal mechanical stresses and strains, aortic volume flow and left ventricular pressure and volume. In chapter 3 the relations between these quantities and different others are mentioned and often simplified in order to reduce the total number of quantities, which must be incorporated in the model. As a consequence of the introduction of these relations several quantities of seemingly secondary importance are introduced in the model, such as the height and torsion angle of the left ventricle, the instantaneous state of local myocardial contraction and subdivision of the local coronary tissue perfusion in the arterial, capillary and venular subcompartments of the coronary vasculature. The height of the cylinder is the equivalent of the base to apex distance in the left ventricle. The torsion angle of the cylinder is the equivalent of the angle over which the base is rotated around the axis of the left ventricle with respect to the apex.

All quantities mentioned above are time dependent, because they vary during the cardiac cycle. The obvious method of describing this system is to characterise the model by a system of first order differential equations with respect to time. In the applied system of $m$ differential equations the instantaneous derivatives of all $(m)$ characteristic quantities $y_{i}$ with respect to time are

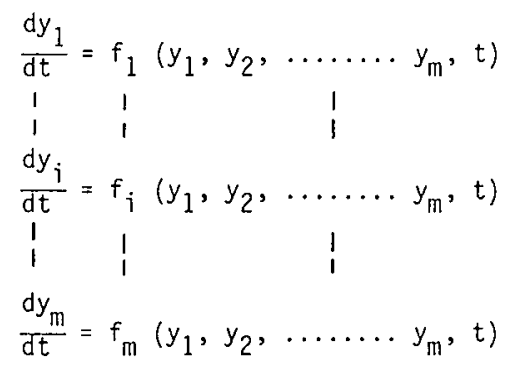


A system of differential equations which can be described in the form of (4.1.1) can be solved numerically by various methods. In the present model a fourth-order Runge Kutta method with fixed time step value $\Delta t$ is chosen ${ }^{1)}$. In order to apply this method at a given time, defined by the time variable $t=t_{0}$, all values of $y_{j}$ must be known. The values of $y_{j}$ at $t=t_{0}$ are the initial conditions of the set of differential equations. In chapter 9 these initial conditions are discussed. In solving the system of equations the values of all quantities $y_{i}$ are computed numerically, starting from $t_{0}$ at intervals $\Delta$.

If no special precautions are taken a stable numerical solution requires mutual independency of the quantities $y_{i}$. The best strategy to avoid mutua? dependence is to introduce a minimum number of quantities $y_{j}$, which characterise the instantaneous situation sufficiently to derive all quantities of interest from the characteristic quantities $y_{i}$. A logical consequence of this strategy is that the characteristic quantities $y_{i}$ do not necessarily correspond to the quantities of interest. In section 4.2 the quantities $y_{i}$ are mentioned and in section 4.3 some important related physiological quantities. The method of computation of the derivatives is schematically presented in section 4.4 .

\subsection{Characteristic quantities of the instantaneous situation}

As a result of the strategy mentioned in section 4.1 a set of characteristic quantities $y_{j}$ is found to describe the instantaneous state of the left ventricle completely. The description of the system by the quantities $y_{i}$ is convenient for solving the differential equation (4.1.1). However, to elucidate the meaning of the characteristic quantities $y_{i}$, each characteristic quantity also is represented by another symbol which gives the physical meaning of that quantity. Together with the physical symbols the characteristic quantities are listed below:

- volume of the left ventricular cavity $\left(V_{1 v}\right)$

- pressure over the capacitance in the model of the aortic input impedance, the arterial pressure ( $p_{\text {art }}$ )

- volume inflow into the large coronary arteries $\left(q_{c e}\right)$

- volume of the large coronary arteries ( $\left.V_{c e}\right)$ and in each subcylinder:

- sarcomere length under zero load condition ( $1_{s a, j}$; chapter 5)

- delay of the relaxation of the local myocardium ( $\tau_{j}$; chapter 5 ) 
- volume of arteriolar vasculature $\left(V_{c a, j}\right)$

- volume of capillary vasculature $\left(V_{c c, j}\right)$

- volume of venular vasculature $\left(V_{c v, j}\right)$.

In the latter five symbols $j$ represents the ordinal number of the relevant subcylinder. In case of subdivision of the thick walled cylinder in $n$ subcylinders (section 3.2.3) the number of characteristic quantities equals $m=5 n$ +4 .

\subsection{Some important related quantities}

In section 4.2 the characteristic quantities $y_{i}$ are reviewed. Some important related quantities can be directly derived from these characteristic ones. The block-diagram of fig. 4.1 shows a schematical representation of the calculation of the quantities necessary for the computation of the derivatives of $y_{i}$ with respect to time.

In the first calculation block (chapter 7) the local stresses, sarcomere lengths, intramyocardial pressures, left ventricular pressure and dimensions of the cylinder are calculated on the basis of

- quantities, which characterise the state of muscle contraction in each subcylinder ( $1_{s a, j}$ and $\left.\tau_{j}\right)$

- total volume of the arteriolar, capillary and venular vasculatures in each subcylinder $\left(V_{c a, j}, V_{c c, j}\right.$ and $\left.V_{c V, j}\right)$

- volume of the left ventricular cavity $\left(v_{1 v}\right)$.

In the same calculation block aortic volume flow and ascending aortic pressure are derived from the left ventricular pressure and the arterial pressure ( $\mathrm{P}_{\text {art }}$; chapter 8 ).

In the second calculation block (chapter 6) in each subcylinder volume flow from the large coronary arteries into the arterioles, from the arterioles into the capillaries, from the capillaries into the venules and from the venules into the veins and right atrium are calculated from

- volume of the large coronary arteries $\left(V_{c e}\right)$

- local intramyocardial pressure

- local volume of the arterioles $\left(V_{c a, j}\right)$

- local volume of the capillaries $\left(V_{c c, j}\right)$

- local volume of the venules $\left(V_{c v, j}\right)$

- local sarcomere length.

The volume of a vasculature determines the transmural pressure differences over the vessel walls and the resistance of the vasculature. Intravascular 

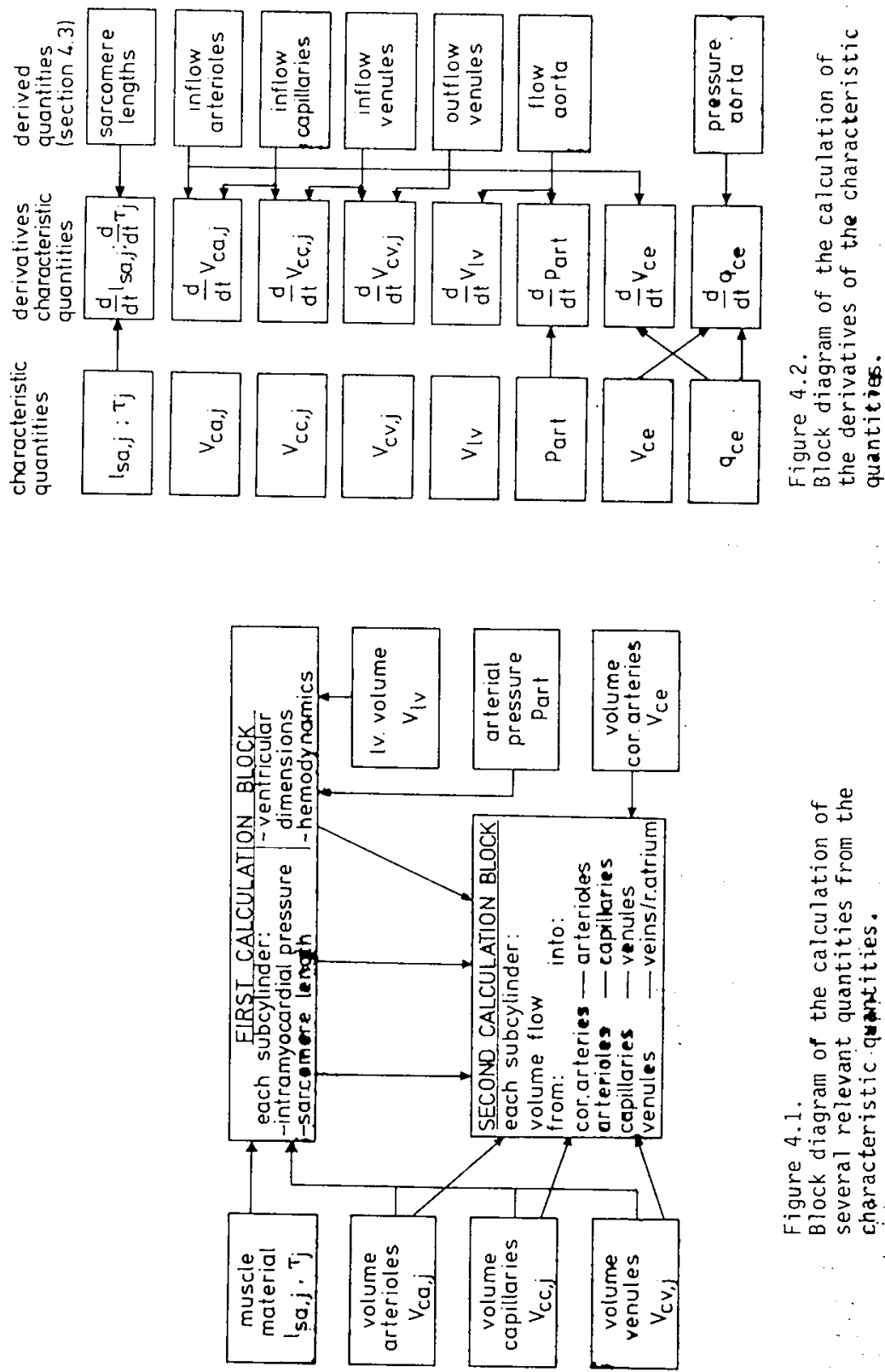

$4 \frac{9}{5}$

동 통

$\pm 4$

至

空嵒

元类

U十

ه 달

开向

$4+0$

0

E $>$

空里

$\rightarrow$ on

$\therefore 2$

宁可

ᄂ

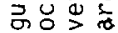
능 至 
pressure equals the sum of pressure over the vessel wall and intramyocardial pressure. Flow is determined by intravascular pressure gradient and flow resistance. Local sarcomere length is supposed to be proportional to the average length of the capillaries, since the capillaries run parallel to the myocardial fibers.

\subsection{The computation of the derivatives of the characteristic quantities}

The derivatives of the characteristic quantities are determined by the characteristic quantities and time, which implies that the system of differential equations satisfies relation (4.1.1). In a block-diagram (fig. 4.2) the caculation of these derivatives is presented schematically. The derivatives of the characteristic quantities which control the state of muscle fiber contraction in each subcylinder are calculated from the related characteristic quantities and the local sarcomere length, according to the relations presented in chapter 5 .

The derivative of arterial pressure depends on both arterial pressure and aortic flow (chapter 7).

The derivatives of the volumes in the arterioles, capillaries and venules in each subcylinder are equal to the difference between inflow and outflow of each related subcompartment (chapter 6). The derivative of the volume flow into the large coronary arteries depends on this volume flow, the aortic pressure and the volume of the epicardial coronary arteries (chapter 6 ). The derivative of the volume of the large coronary arteries equals the difference between the volume flow into the coronary arteries and the total volume flow into the arterioles (chapter 6).

\section{Literature}

1. Abramowitz, W., Stegun, I.: Handbook of mathematical functions, p. 896. Dover Publ. New York (1970). 
5. THE STRESS-STRAIN RELATION OF THE MYOCAROIAL MATERIAL

5.1. Introduction.

The relation between stress and strain in myocardial material has to be known in order to derive the left ventricular pressure from the left ventricular volume in the present model. The myocardium consists mainly of striated muscle. However, most of the data concerning striated muscle are obtained from skeletal muscle. Therefore, contractile properties of the myocardium are derived from the contractile properties of skeletal muscle by introducing some modifications, which are specific for the myocardium. In section 5.2 skeletal muscle and myocardium are compared. Also several approaches to describe the mechanical properties of myocardial material are discussed. In section 5.3 the relation between stress, strain, strainrate and time is presented applying a mathematical model. In section 5.4 experiments on myocardial material behavior, as reported in literature, are compared with a computer simulation of these experiments. This computer simulation is based upon the relation described in section 5.3. In the sections 5.5 and 5.6 isotropic and anisotropic myocardial material properties are discussed, respectively. In section 5.7 left ventricular performance is evaluated, assuming isotropic, as well as anisotropic behavior of the myocardial tissue.

\subsection{Approach to describe the mechanical properties of myocardial material}

Striated muscle material is composed of muscle fibers (fig. 5.1), which are the muscle cells. A muscle fiber is composed of a series of contractile units, known as sarcomeres ${ }^{46)}$. A sarcomere contains fibrils consisting of actine and myosine filaments (fig. 5.2). The actine filaments are connected to the $\mathrm{Z}$ discs at one end and overlap the myosine filaments at the other end. The myosine filaments are thicker and form a hexagonal structure. In the overlap zone the actine filaments are hexagonally arranged in the interspaces of the myosine filaments. During contraction the actine filaments slide along the myosine filaments, which results in sarcomere shortening ${ }^{22}$ ). There is convincing evidence that the force is generated by interaction of the actine and myosine filaments 22,23$)$. Some properties of myocardial tissue are virtually similar to those of skeletal muscle, for instance:

- a contraction of a muscle cell is initiated by electrical depolarization of the cell membrane 37 )

- a single action potential is followed by a single contraction (twitch), 


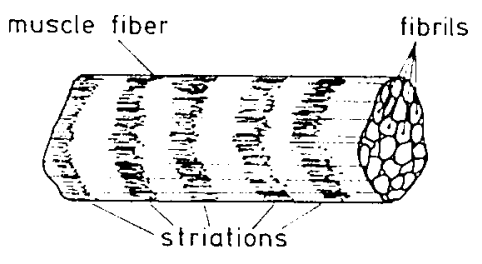

Figure 5.1 .

A striated muscle fiber.

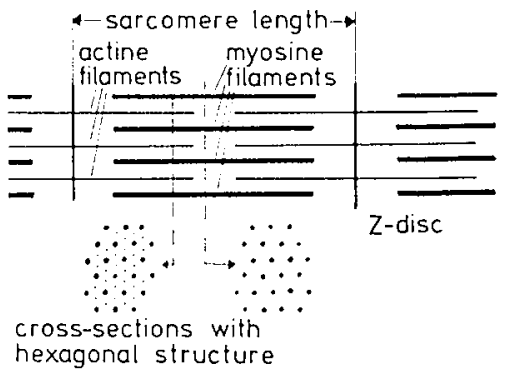

Figure 5.2.

Schematical representation of a sarmere.

characterized by a rapid increase in stress, reaching a maximum, and followed by a rather slow decrease 20 )

- the relation between stress and strainrate, which characterises the contractile mechanism, can be described sufficiently accurately by Hill's equation $^{19)}$.

However, some properties of the myocardial tissue are different from those of skeletal muscle, for instance:

- activation of the myocardium is followed by a longer refractory period, during which reactivation is impossible. Repeated stimulation of myocardial tissue does not result in a tetanus like in skeletal nuscle ${ }^{8}$ ), since in general the refractory period is longer than the duration of the twitch

- at end-diastolic length non-activated cardiac muscle can bear a considerable stress, whereas skeletal muscle is freely extensible in the range of normal length ${ }^{46)}$

- in the myocardium propagation of a depolarization wave occurs through direct contact between the cells 37,46$)$, whereas in skeletal muscle activation occurs per cel1 33 )

- cardiac cells have many cross-connections.

Most of the attempts to describe the mechanical behavior of myocardial tissue are based upon known facts about skeletal muscle, adapted to some properties of myocardial tissue. The contractile behavior of a tetanized nuscle can be described sufficiently accurately by a first order differential equation, relating stress, strain and strainrate ${ }^{19}$ ). However, since in the myocardium the state of contraction also depends on time, the latter has also to be accounted 
for in the differential equation.

Various approaches to characterise the contractile properties of myocardial material have been proposed, i.e.

- a phenomenological approach, mainly based upon experimental data

- a more basical approach, founded on a hypothesis concerning the molecular basis of the mechanism of contraction

- a modelling approach, based upon a mathematical model, which fits experimental data as close as possible.

In a phenomenological approach the relation between stress, strain, strainrate and time is determined under various experimental circumstances $4,5,6,16,36$ ). The results obtained are substituted into a model of left ventricular pump function ${ }^{40)}$. However, by the nature of this approach it is difficult to get insight in the relation. Changes in the performance of the contraction are difficult to simulate, since in this case a new experimentally determined stress-strain-strainrate-time relation is required.

On the other hand a more basic approach has also disadvantages. Several contraction mechanisms have been proposed, such as the cross-bridge theory ${ }^{15,23)}$, the electrostatic theory 24,34$)$ and the hydrodynamic theory ${ }^{50)}$, but none of them explains cardiac muscle properties sufficiently well. The cross-bridge theory is most widely accepted, but recently this theory became subject of discussion 34). A contractile mechanisril, which is based on the principles of the electrostatic theory, looks simpler and can also explain many properties of the contraction of muscle. However, the latter theory is not investigated and discussed as critically as the cross-bridge theory is. The hydrodynamic theory is only mentioned in an abstract ${ }^{50}$ ) and not well-known. Thus the mechanism of contraction is not yet clear and consequently mathematical relations based upon hypothetical contraction mechanisms remain speculative. In the present model of the left ventricle a compromise is found in a modelling approach. A relatively simple mathematical relation between stress, strain, strainrate and time is determined, which agrees fairly well with experimental results, as reported in the literature. Changing cardiac muscle performance can be described by changing only a few parameters in the model.

\subsection{Model of the stress-strain-strainrate-time relation of cardiac muscle}

\subsubsection{Introduction}

The present section deals with the relation between stress, strain, strain- 
rate and time in cardiac muscle. Before passing on to the problem of finding a suitable relation (subsection 5.3.2) several quantities, concerning this relation, have to be defined and, if necessary, to be translated into quantities, which are easier to handle.

From theoretical mechanics it is known that stress and strainrate are tensors, each of them consisting of 6 independent components. After transformation of the tensors on the principal directions the shear componerts vanish and consequently the three remaining principal strainrates, as well as the three principal stresses coincide with the coordinate axes. It can be shown that in case of a state of tensile stress, where only one principal stress is present, the corresponding large strain is determined by the instantaneous value of that stress.

In the myocardial material one principal direction (the 1-direction) is parallel to the muscle fibers, the other two principal directions (the 2- and 3directions) are perpendicular to the fiber direction (fig. 5.3). In experiments to determine the contractile behavior of muscle material the muscle fibers are assumed to run in parallel. In this case an experimental relation is found between the stress $\sigma_{1}$ and strain $\varepsilon_{1}$ in the fiber direction for the situation of purely tensile loading.

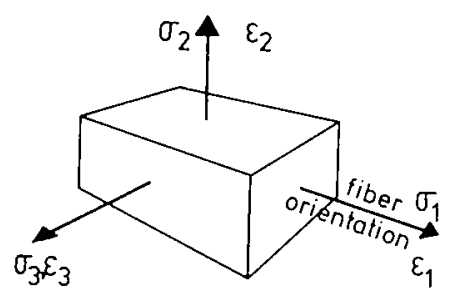

Figure 5.3.

Components of stress and strain in the principle directions.

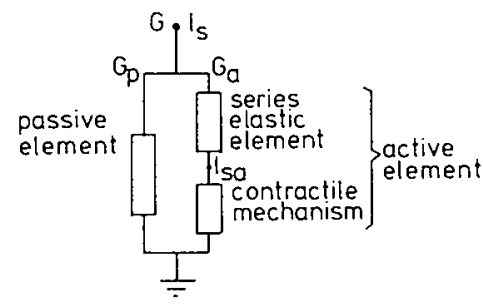

Figure 5.4 .

Description of cardiac muscle by a passive and an active element in parallel. The active element is composed of an elastic element in series with the contractile element.

In the present section the relation between $\sigma_{1}$ and $\varepsilon_{1}$ will be modelled in a system of mathematical relations. To this purpose the situation of tensile loading $\left(\sigma_{2}=\sigma_{3}=0\right.$ ) will be assumed. This situation is also present in papillary muscle in the commonly used set-up to study the in vitro contractile 
behavior of cardiac muscle. For the sake of convenience the stress $\sigma_{1}$ and natural strain $\varepsilon_{1}$ will be replaced by the force per sarcomere volume $G$ and sarcomere length $l_{s}$ according to

$$
\begin{aligned}
& G={ }_{1} / 1_{5} \\
& I_{5}=\exp \left(\varepsilon_{1}\right) \cdot 1_{\text {ref }}
\end{aligned}
$$

where $1_{\text {ref }}$ is defined to be the sarcomere length for $c_{1}=0$. The quantity $G$ is given preference over $\sigma_{1}$ since the sarcomere volume is virtually invariant ${ }^{18)}$ during contraction, whereas the cross-sectional area varies substantialiy. Energy per unit of volume is an important quantity and is obtained by integrating stress o with respect to strain $\varepsilon_{1}$. In an analogous way as $\varepsilon_{1}$ is complementary to ${ }_{1}$, sarcomere length $l_{S}$ is complementary to force per sarcomere volume $G$ and therefore the quantity $I_{S}$ is preferable to the quantity $\varepsilon_{1}$. In this context it is important to mention that the strain of a sarcomere must be equal to the strain of the local myocardial tissue. This is confirmed by experiments 27,30$)$ which demonstrate that marks on muscle fibers move synchronously with the sarcomeres. A logical consequence of the replacement of the combination of stress and strain by the combination of force per sarcomere volume and sarcomere length is that strainrate is replaced by its analogue velocity of sarcomere shortening $v$, according to

$$
v=-\frac{d l_{s}}{d t_{s}}=l_{\text {ref }} \exp \left(\varepsilon_{1}\right) \frac{d \varepsilon_{1}}{d t}
$$

The start of depolarization of the cells in the myocardial tissue under investigation is defined as $t_{s}=0$. The symbol $t$ is reserved for the time variable of the complete model. Because activation of the different subcylinders is asynchronous, each subcylinder has its own value of $t_{s}$.

\subsubsection{The dependence of force per sarcomere volume on sarcomere length 2 velocity of sarcomere shortening and time}

Commonly the mechanical behavior of a sarcomere is characterized by a passive elastic element parallel to a contractile mechanism. Probably passive and contractile properties are not as separate as this characterization suggests. However, for simplicity of the mathematical description, this common characterization of a sarcomere is used. When the muscle is not activated, the 
force-length relation is controlled by the passive elastic element. During contraction the force generated by the contractile mechanism $G_{a}$ is added (fig. 5.4 ) to the force $G_{p}$ accounting for the elastic properties of the passive element, so that

$$
G=G_{p}+G_{a}
$$

In describing the mechanical properties of the passive element, this system is usually characterized by a purely elastic element with an exponential forcelength relation ${ }^{13}$ ). Unactivated cardiac muscle has visco-elastic properties 35 ). In the present study, however, the viscous behavior of unactivated cardiac muscle is neglected. Thus for the passive element the relation between force per sarcomere volume $G_{p}$ and the sarcomere length $]_{s}$ is assumed to be

$$
G_{p}=G_{p o}\left(\exp \left[b\left(1_{s}-a\right)\right]-1\right)
$$

where $a, b$ and $G_{p o}$ are parameters. The parameter a represents the sarcomere length under zero load $\left(G_{p}=0\right)$. In experiments on heart muscles the sarcomere length during the non-activated state is seldom found less then $1.96 \mu \mathrm{m} 11,41$, $47,52)$. If the length is less buckling of the muscle occurs and the sarcomeres do not shorten anymore. In the model, therefore, it is taken a $=1.95 \mathrm{\mu m}$. The parameter $b$ governs the exponential increase of $G_{p}$ with increasing sarcomere length. From experiments on cat papillary muscle ${ }^{2}, 12,25,27,28,31,35,38,45,49$, 52) it could be concluded that the value of $b$ is ranging from 6 to $22 \mathrm{~m}^{-1}$ at temperatures, varying from $20^{\circ} \mathrm{C}$ to $37^{\circ} \mathrm{C}$. Most values of $b$, obtained from these experiments, are close to $8 \mathrm{um}^{-1}$. For the present this value of $b$ is introduced in the model, being aware of the fact that this value may be different at $37^{\circ} \mathrm{C}$. The parameter vaiue $G_{p o}$ is obtained from experimental data on $G_{p}$ at a given sarcomere length $\left(\mathrm{e} . \mathrm{g} .1_{\mathrm{s}}=2.1 \mathrm{\mu m}\right)$. After substitution of the values of $a$ and $b$ into relation $(5.3 .5), G_{\text {po }}$ can be found. Since the force generated by the passive element is increasing rapidly with increasing sarcomere length, the value of $G_{p o}$ is not very critical. This is illustrated by the fact, that $0.07 \mathrm{Hm}$ change in sarcomere length doubles the force of the passive element. A commoniy accepted value of the passive stress at a sarcomere length $1_{\max }$ with maximum active stress amount to $\left.10^{4} \mathrm{~N} \mathrm{~m}^{-2} 17,27,45,52\right)$, leading to a value of $G_{p o}=4.43 \cdot 10^{8} \mathrm{~N} \mathrm{~m}^{-3}$. In the experimental set-ups used, the sarcomere length corresponding to $1_{\max }$ is approximately equal to $\left.2.25 \mathrm{\mu m} 27,45,46\right)$. 
The active element is supposed to consist of an element with an internal length $1_{\text {sa }}$ in series with a series elastic component with length $1_{s s}$.

$$
r_{s}=1_{s a}+1_{s s}
$$

At zero load $I_{s s}$ is defined to be zero, implying that the inmeasurable internal length of the contractile mechanism is equal to the measureable sarcomere length at zero loading. The internal length $1_{\text {sa }}$ is considered to be a characteristic quantity (chapter 4), which cannot vary instantaneously. The instantaneous value of the force per unit sarcomere volume generated by the active element $G_{a}$ is determined by the characteristics of the series elastic component and its length. Pollack et al. ${ }^{41)}$ found, that sudden untoading of cardiac muscle changed instantaneously the sarcomere length $1_{s}$ by an amount of approximately 0.04 ilil. In the modelling approach this sudden shortening of $I_{s}$ can only be due to a change of length of the series elastic element. For many applications, like Pollack et al. ${ }^{41,42)}$ suggested, the series elasticity may be neglected, since the maximum changes of $1_{s s}$ are small compared to the sarcomere length. For mathematical convenience, however, the series elastic component is inserted in the model. It is easier to consider the muscle material as an elastic material rather than as a viscous material. In the latter case the structure of the differential equation mentioned in chapter 4 changes essentially and, concerning the choice of myocardial tissue properties, the flexibility of the model deteriorates. As will be shown later in this section, the characteristics of the series elastic component are chosen in such a way that unloading of the myocardial tissue during isometric contraction changes the sarcomere length by $0.04 \mathrm{~km}$.

The contractile mechanism is characterized by a length $1_{\text {sa, }}$, a force per sarcomere volume $G_{a}$, a rate of shortening $v_{a}$ and a state of activation $A$. The quantities $1_{\text {sa }}$ and $v_{a}$ are related by

$$
v_{a}=-\frac{d l_{s a}}{d t_{s}}
$$

Since $l_{s a}$ is a characteristic quantity (chapter 4 ), its derivative $-v_{a}$ must be known to solve the differential equation (4.1.1). In the present section the method of calculation of $v_{a}$ is given. At a certain degree of activation $A$ the relation between $G_{a}$ and the rate of shortening $v_{a}$ is assumed to be described 
by Hill's 19) equation, which can be written as

$$
\left(1+\frac{G_{a}}{G_{a 0}}\right)\left(1+\frac{v_{a}}{v_{\text {ao }}}\right)=A+1
$$

where $G_{a 0}$ and $v_{a 0}$ are parameters. In fig. 5.5 relation (5.3.8) is shown graphically for different values of $A$. Experimental data on this relation, as reported by Edman ${ }^{9}$ ) are shown in fig. 5.13. During a contraction of myocardial tissue $A$ depends on $I_{s a}$ and $t_{s}$. Generally, active force during isometric contraction, which is directly related to $A$, is assumed to depend on the sarcomere length $1_{s}$. Since $1_{s}$ and $1_{s a}$ have about the same value, $1_{s a}$ is used as variable instead of $I_{s}$. The advantage of the use of $l_{s}$ as variable is that $1_{\text {sa }}$ belongs to the contractile mechanism.

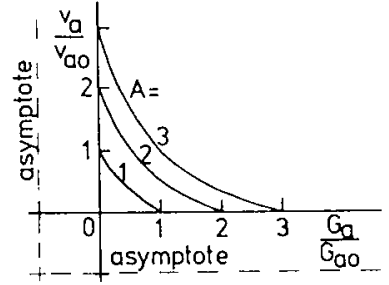

Figure 5.5 .

The relation between velocity of shortening and force of a skeletal muscle according to Hill.

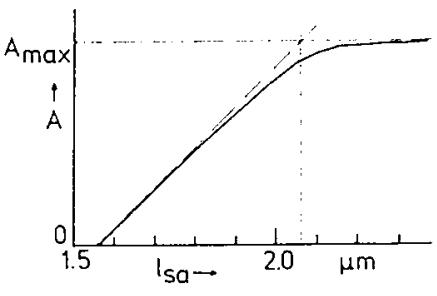

Figure 5.6.

Proposed relation between maximum activation and sarcomere length.

$$
\text { Defining } \begin{aligned}
G_{\max } & =G_{a} \text { at } v_{a}=0, \\
v_{\max } & =v_{a} \text { at } G_{a}=0 .
\end{aligned}
$$

and substituting this into relation $(5.3 .8)$ renders

$$
\begin{aligned}
& G_{\text {max }}=A G_{a o} \\
& v_{\max }=A v_{a o}
\end{aligned}
$$

Experiments of Pollack et al. $\left.{ }^{41}\right)$ show, that in the physiological range (1.6 um $<I_{\text {sa }}<2.2 \mathrm{\mu m}$ ) the ratio of $G_{\max }$ and $v_{\max }$ is independent of the sarcomere length. Moreover, Edman et al.10) showed that this ratio does not vary in a large part of the contraction cycle. These results indicate, that both $G_{a 0}$ and 
$v_{\text {ao }}$ can be regarded to be constant. Changes of $G_{\text {max }}$ and $v_{\text {max }}$ are proportional to the activation $A$.

In the present modelling approach the activation $A$ is supposed to depend on time as well as on sarcomere length. This dependency on two variables is mathematically expressed by:

$$
A=A_{1}\left(l_{s a}\right) \cdot A_{2}\left(t_{s}\right)
$$

where $A_{1}$ only depends on $1_{s a}$ and $A_{2}$ on $t_{s}$. The maximum value of $A_{2}\left(t_{s}\right)$ is normalized to unity. The function $A_{1}\left(l_{s a}\right)$ can be deternined from results obtained from isosarcometric contractions as performed by Pollack et al. ${ }^{41)}$. In experiments on the mechanical behavior of muscles several investigators $7,30,41$ ) found that the liuscle length and sarcomere length do not vary synchronously. During isometric contraction sarcomeres in the healthiest parts of the muscle appeared to shorten at the cost of elongation of those sarcomeres, which were dallaged by the clamping of the muscle in the experimental set-up. Therefore, experiments on muscle can only be qualitatively extrapolated to experiments at sarcomere level. Since quantitative results of experiments at sarcomere level are very rare, references to sarcomere controlled contractions are limited to the survey of Pollack et al. 41). They found, that during maximum contraction both $G_{\max }$ and $v_{\max }$ were proportional to $\left(I_{S}-1.6 \mathrm{~mm}\right)$. This is confirmed by experiments of Sonnenblick et al. ${ }^{44)}$ on isometric contraction. At sarcomere lengths over 2.1 um stress appeared to level off to a plateau. According to the relations (5.3.9) and (5.3.11) it is concluded that $A_{1}\left(1_{s a}\right)$ is proportional to the experimental function $G_{\max }\left(7_{\mathrm{sa}}\right)$, yielding an experimental relation:

$$
\begin{aligned}
& A_{1}\left(1_{s a}\right)=A_{\max }\left(1+x-\sqrt{\left.x^{2}+0.01\right)}\right. \\
& \text { with } x=\frac{l_{s a}-2.06 \text { um }}{1 \text { um }}
\end{aligned}
$$

where the length of the series elastic element is assumed to be equal to 0.04 uml $^{41)}$. In fig. 5.6 this relation is shown graphically.

values of $A$ are obtained by fitting experimental force-velocity data to a hyperbola according to relation (5.3.8). The value of $A$ appears to depend on the experimental set-up used. In skeletal muscle Hill ${ }^{18}$ ) ano Julian ${ }^{26)}$ found a value $A_{\max }=4$. Edman et al. ${ }^{9,10}$ obtained force-velocity data from rabbit 
papillary muscle under various circumstances. The value of A appeared to decrease with increasing temperature, which is confirmed by experiments of Binkhorst et al. 1) on human skeletal muscle. In the experiments of Edman et al. ${ }^{9}$, 10) on rabbit papillary muscle it was found that $\mathrm{A}=4$ at $27.5^{\circ} \mathrm{C}, \mathrm{A}=2.4$ at $31^{\circ} \mathrm{C}$ and $\mathrm{A}=1.5$ at $37^{\circ} \mathrm{C}$. The latter value of $\mathrm{A}$ corresponds very well with the experiments of Ross et a 1.43 ). From his experiments on the left ventricle of the $\operatorname{dog}$ it can be derived that $A=1.6$ at this temperature. At lower instantaneous values of $G_{\max }$ Edman et al. ${ }^{9)}$ found lower instantaneous values of A. This indicates that the force-velocity relation during the contraction cycle is controlled by changes in the parameter $A$, as will be discussed in the present chapter. For the present the value of $A_{\max }$ in the experiments of Pollack et al. 41 ) is assumed to be equal to 4 .

values of $G_{a 0}$ and $v_{a 0}$ are obtained from experimental values of $G_{m a x}$ and $v_{\max }$ using relations (5.3.9) and (5.3.10). In an experimental set-up, where sarcomere length was measured directly ${ }^{41)}$, rat papillary muscle at $26^{\circ} \mathrm{C}$ could generate a stress of $1.1 \cdot 10^{5} \mathrm{~N} \mathrm{~m}^{-2}$ at $l_{\mathrm{s}}=2.1 \mathrm{wm}$, which corresponds to a $G_{\max }=5.2 \cdot 10^{10} \mathrm{~N} \mathrm{~m}^{-3}$. The stress developed during an isometric contraction is practically independent of temperature 1,9$)$, whereas maximum rate of shortening $v_{\max }$ depends on temperature. Edman et al. ${ }^{9)}$ found in rabbit papillary muscle experiments that $v_{\max }=5.5 \mu \mathrm{m} \mathrm{s} \mathrm{s}^{-1}$ at $26.5^{\circ} \mathrm{C}, v_{\max }=6.3 \mu \mathrm{m} \mathrm{s}^{-1}$ at $27.5^{\circ} \mathrm{C}$ and $v_{\max }=9.9 \mathrm{~km} \mathrm{~s} \mathrm{~s}^{-1}$ at $31.0^{\circ} \mathrm{C}$. In cat papillary muscle Brutsaert et al. 5) found that $v_{\max }=7.6 \mathrm{~m} \mathrm{~s}^{-1}$ at $29^{\circ} \mathrm{C}$, which suggests that velocity of shortening in cat papillary muscle and rat papillary muscle are not significantly different. In the experiments of Pollack et al. ${ }^{41}$ ) on rat papillary muscle at $26^{\circ} \mathrm{C}$ a value of $v_{\max }=10$ un $\mathrm{s}^{-1}$ was found, which value after correction for temperature is significantly higher than the values found by Edman et al. ${ }^{9}$ ) and Brutsaert et al. ${ }^{5)}$. In the model of the contractile mechanism the data of Pollack et al. ${ }^{41)}$ are applied because they measured directly the sarcomere length in their experiments. This results in

$$
\begin{aligned}
& G_{a 0}=1.4 \cdot 10^{10} \mathrm{~N} \mathrm{~m}^{-3} \\
& v_{a 0}=2.9 ; \mathrm{s} \mathrm{s}^{-1}
\end{aligned}
$$

One should realise, however, that the values of $G_{20}$ and $v_{a 0}$ both depend on such variables as the type of muscle, temperature ${ }^{1,9,10,14)}$ and $\mathrm{Ca}^{2+}$-concentration ${ }^{3,5)}$. Therefore, in the model of the left ventricle these parameters 
must be estimated by comparing the hemodynamic characteristics of the simulated left ventricle to those of the left ventricle in the biological situation.

As shown by Pollack et al. ${ }^{41)}$ the series elastic element changes its length maximally by an amount of $0.04 \mathrm{~km}$. Assuming that the instantaneous sarcomere shortening during instantaneous unloading of the muscle amounts to $0.04 \mathrm{um}$ the following reiation between force and length can be used:

$$
G_{a}=\left(1_{s}-1_{s a}\right) \frac{G_{a o} \cdot A_{1}\left(1_{s a}\right)}{0.04 \cdot m}
$$

By applying relation (5.3.8), equation (5.3.7) is transformed into the following differential equation which determines the derivative of $1_{\mathrm{sa}}$ :

$$
\frac{d l_{s a}}{d t_{s}}=\left\{1-\frac{1+A_{1}\left(l_{s a}\right) \cdot A_{2}\left(t_{s}\right)}{1+\left(\frac{G_{a}}{G_{a 0}}\right)}\right\} v_{a 0}
$$

The initial value of $l_{s a}$ at $t_{s}=0$ is equal to the sarcomere length $l_{s}$ at $t_{s}$ $=0$.

The time dependence of the force is expressed by $A_{2}\left(t_{s}\right)$ in relation (5.3.11). The function $A_{2}\left(t_{s}\right)$ is derived from the force-time relation during an isosarcometric contraction. Under these circumstances relation (5.3.8) can be transferred to

$$
G_{a}\left(t_{s}\right)=G_{a 0} A_{1}\left(1_{s a}\right) A_{2}\left(t_{s}\right)
$$

Since the factor $G_{a O} A_{1}\left(l_{s a}\right)$ is approximately constant during an isosarcometric contraction, the function $A_{2}\left(t_{s}\right)$ corresponds the course in time of $G_{a}\left(t_{s}\right)$. The course of an isosarcometric contraction as a function of time has not been extensively examined, except by Pollack et al. ${ }^{41}$ ). For this reason, data are taken from reports on isometric contractions of pieces of cardiac muscle $2,4,16$, $29,32,39)$. During isometric contraction of myocardial material, force increases rapidly to a maximum, levels off to a more or less distinct plateau and next gradually decreases to zero. In a mathematical simulation, $A_{2}\left(t_{s}\right)$ is assumed to be the product of a function $g_{l}\left(t_{s}\right)$, with the initial value $g_{1}(0)=0$, increasing rapidly to unity, and $g_{2}\left(t_{s}\right)$, which equals unity for low values of $t_{s}$ and decreases relatively slowly to zero for increasing values of $t_{s}$.

The increased duration of the twitch with increased sarcomere length ${ }^{32,39)}$ is 
simulated by an increased delay $\tau$ of $g_{2}\left(t_{s}\right)$. During a non-isometric contraction the delay $\tau$ is decreased. Meiss et al. ${ }^{32}$ ) found that the decrease of $\tau$ is the larger the later the muscle shortening occurs. Therefore $\tau$ is assumed to vary with the time $t_{s}$ and to depend on the shortening of the muscle as described below. The following mathematical concept describing the time dependency of the function $A_{2}\left(t_{s}\right)$ is assumed:

$$
A_{2}\left(t_{s}\right)=g_{1}\left(t_{s}\right) g_{2}\left(t_{s}-\tau\right)
$$

with

$$
\begin{aligned}
& g_{1}\left(t_{s}\right)=1-\left\{1+\left(\frac{t_{s}}{\tau_{s}}\right)^{4}\right\}^{-1} \\
& g_{2}\left(t_{s}\right)=\left\{1+\exp \left(\frac{t_{s}-\tau}{\tau_{s} 2}\right)\right\}^{-1}
\end{aligned}
$$

where $\tau_{s 1}$ and $\tau_{s 2}$ are parameters governing the risetime and the decay time of the twitch respectively. An impression of the function $A_{2}\left(t_{s}\right)$ can be obtained from the time course of the force in the simulated isosarcometric contraction, represented by the dashed line in fig. 5.8. The differential equation determining the value of $\tau$ is

$$
\frac{d \tau}{d t_{s}}=\min \left(0, b_{1} t_{s} \frac{d l_{s a}}{d t}\right)+\frac{\frac{d l_{s a}}{d t}}{\left(1+\frac{t_{s}}{\tau_{s 1}}\right) v_{s l}}
$$

where $b_{1}=$ parameter which governs the decrease in $\tau$ according to the moment of shortening 32 )

$$
\begin{aligned}
v_{s 1}= & \text { parameter which governs larger values of } \tau \text { at larger initial sarco- } \\
& \text { mere length } 32,39)
\end{aligned}
$$

The minimum value criterium is used since during relaxation changes in sarcomere length seem less relevant. The second term in relation (5.3.19) expresses the increasing delay $\tau$ with increasing sarcomere length during the first part of the contraction cycle. The initial value of $\tau$ at $t_{s}=0$ satisfies

$$
\tau=\frac{1_{s a}-1_{s 1}}{v_{s 1}}
$$


where $1_{s 1}=$ extrapolated sarcomere length, where $\tau=0$.

For the present the parameter values as listed below are used:

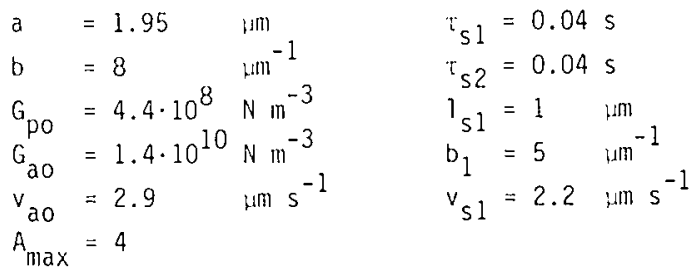

In conclusion: The contractile behavior of myocardial material is characterised by two differential equations. Equation (5.3.16) relates the derivative of the unloaded instantaneous sarcomere length $1_{\text {sa }}$ to the instantaneous values of both the active load $G_{a}$ and $l_{s a}$. Equation (5.3.19) relates the derivative of the delay of the decreasing slope of the activation $T$ to the derivative of $1_{s a}$. At known sarcomere length $1_{s}$ the force can now be computed. As to this the length of the series elastic element $l_{s s}$ is computed according to relation (5.3.6). The active force per sarcomere volume $G_{a}$ is computed fron ${ }_{\text {ss }}$ using relation (5.3.15) which characterises the properties of the series elastic element. The passive force $G_{p}$ is computed from $l_{s}$, using the characteristics of the passive element as described by relation (5.3.5). The total force per sarcomere volume $G$ is the sum of $G_{p}$ and $G_{a}$ as expressed by relation (5.3.4). The derivatives of $l_{\text {sa }}$ and $\tau$ are determined by the relations (5.3.16) and (5.3.19), in which the actual value of $G_{a}$ has to be substituted. Thus the two differential equations in $l_{s a}$ and $\tau$ satisfy the conditions, mentioned in relation to the equation (4.1.1), concerning solvability of the system of differential equations.

\subsection{Computer simulation of experiments on cardiac muscle}

In the present section experiments on papillary muscles ${ }^{5}$ are simulated in the model, as described in section 5.3. In these experiments force, length and rate of shortening of a piece of papillary muscle are measured. Initially the muscle is loaded with a preload $F_{p r}$ (fig. 5.7 a), and the length of the muscle is limited to a maximum, which equals the momentary length, viz. prelength. An after-load is added to the preload (fig. 5.7 b) and the muscle is stimulated. The length remains constant until the force generated by the muscle exceeds the load. Then the muscle shortens until the activation decreases to such an 


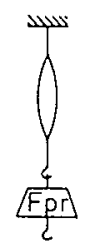

$a$

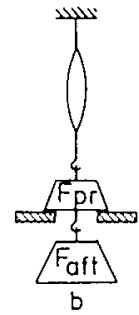

Figure 5.7 .

Schematic drawing of the set-up for experiments on papillary muscle.

Panel a: The muscle is loaded with a preload.

Panel b: The length of the muscle is limited to the length during preload, whereupon an afterload $\mathrm{F}_{\text {aft }}$ is added.

extent, that the muscle starts to lengthen. Lengthening stops, when prelength is reached again. Subsequently force decreases to the preload level again and the contraction is finished.

The various authors $5,9,41)$ used different experimental conditions. Therefore, comparison of the model-simulations with experiments described in the literature is only qualitative. Results of a computer simulation of these experiments are shown in fig. 5.8. The parameter values used are listed in relation (5.3.21). The forceper sarcomere volume $G$, sarcomere length $I_{S}$ and velocity of shortening $v_{s}$ are presented as a function of time. The preload $G_{p r}$ was chosen to be $62 \cdot 10^{7} \mathrm{~N} \mathrm{~m}^{-3}$, resulting in a sarcomere length of $2.06 \mu \mathrm{m}$. The total load, which is the sum of preload and afterload, had the following values: $56 \cdot 10^{9}, 42 \cdot 10^{9}, 28 \cdot 10^{9}, 14 \cdot 10^{9}, 7 \cdot 10^{9}$ and $62 \cdot 10^{7} \mathrm{~N} \mathrm{~m}^{-3}$. In fig. 5.9 experimental results of Brutsaert et al. ${ }^{4)}$ are shown. Besides the longer rise time of the force in the experiments of Brutsaert the curves look similar to the curves, generated by the model. Fig. 5.10 shows plots of ${ }_{s}$ versus 's, calculated by the model and fig. 5.11 shows similar experimental results as described by Henderson et al. ${ }^{16}$ ). In another simulation for different values of the preload the maximum velocity of shortening was plotted as a function of the total load. The results are shown in fig. 5.12. Fig. 5.13 shows similar experimental results found by Edman et al. ${ }^{9)}$. Fig. 5.14 shows the simulated maximum active force, developed during an isosarcometric contraction, as a function of the sarcomere length. Fig. 5.15 shows similar results as obtained from the experiments of Pollack et al. ${ }^{41)}$.

Some differences are present between the contractile behavior of cardiac 

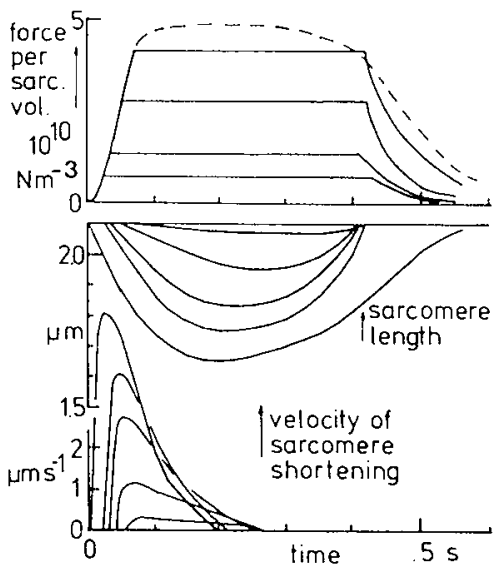

Figure 5.8 .

Computer simulation of a papillary muscle experiment. The upper pane? shows the force developed as a function of time at equal preload, but different afterloads. The middle panel shows the related sarcomere length variations. The lower panel shows the related changes in velocity of sarcomere shortening.
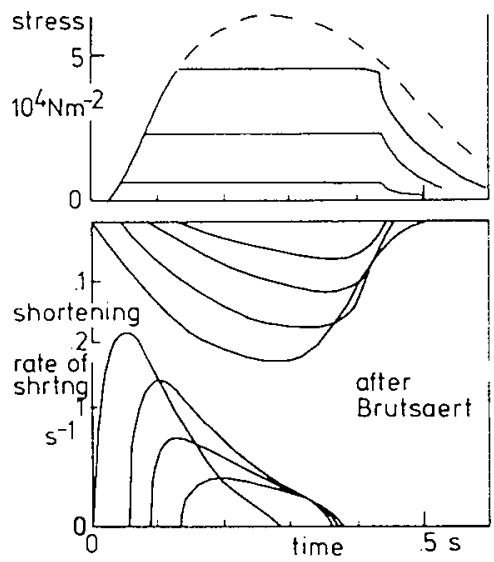

Figure 5.9.

A similar experiment as shown in fig. 5.8, but now performed on papillary muscle iri vitro (Brutsaert 1971).

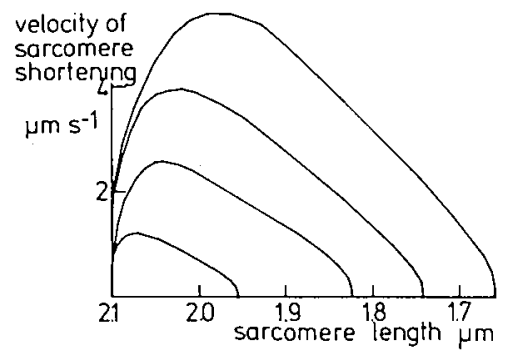

Figure 5.10 .

In the computer simulation shown in fig. 5.8, velocity of sarcomere shortening is plotted as a function of sarcomere length. rate of

shortening

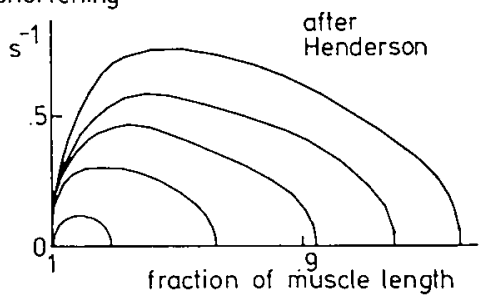

Figure 5.11 .

Velocity of muscle shortening as a function of muscle length, as derived from papillary muscle experiment in vitro (Henderson 1973). 


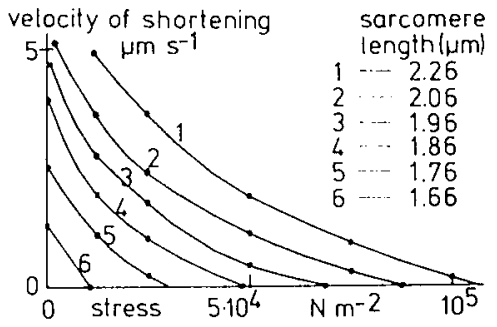

Figure 5.12 .

Maximum velocity of shortening plotted as a function of developed force at various prelengths (computer simulation). The simulation appears to fit Hill's relation fairly well.

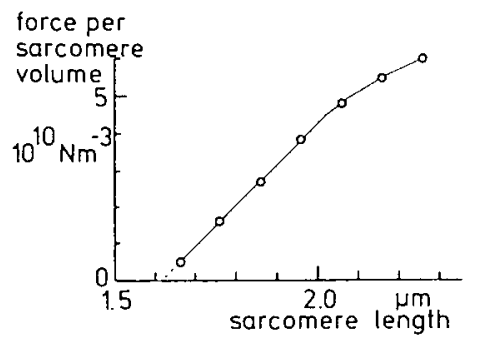

Figure 5.14 .

Maximum active force during an isosarcometric twitch as a function of sarcomere length, generated by the computer simulation.

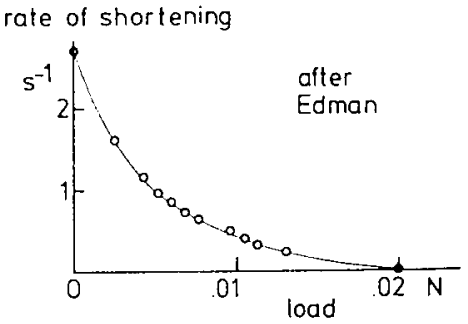

Figure 5.13.

Strainrate as a function of muscle load, as derived from papillary muscle experiments in vitro (Edman 1968).

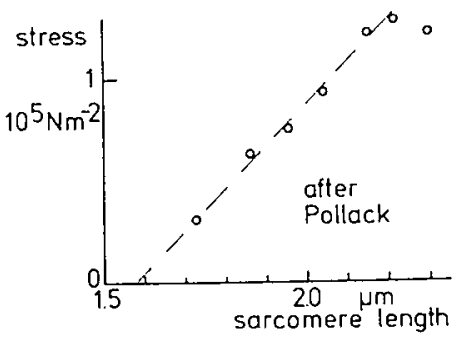

Figure 5.15

laximum active force during an isosarcometric twitch as a function of sarcomere length, as measured by Pollack (1976). 
buscle, as simulated by the model and as found in animal experiments. The most important difference is the slower increase of sarcomere length during relaxation of the cardiac muscle in the simulation. However, the condition of lengthening of cardiac muscle during relaxation as found in in wiro experiments normally does not occur in the $i ;$ yis situation. Left ventricular filling, and consequently lengthening of cardiac muscle occurs during diastole, when there is no activation.

It should be remarked that the paraneters, used in the simulation of cardiac muscle, are obtained from in $v i$ ro experiments on cat, rabbit and rat papillary muscle at various temperatures. Moreover, most of these experiments are performed with the use of an experimental set-up, where unequal shortening of the muscle under investigation could easily occur ${ }^{41)}$. It is likely, that the values of the parameters which describe the behavior of canine myocardial muscle in vixe at $37^{\circ} \mathrm{C}$, are somewhat different. For the present, contractile behavior of cardiac muscle is assumed to be described fairly well by the present model of muscle contraction.

\subsection{Isotropic myocardial material}

In general the mechanical properties of cardiac muscle material is described by an experimentally determined relation between stress and strain in one principal direction. In this section it will be shown, that the necessary additional relations between the stress and strain components can be obtained by the assumption of isotropy of the cardiac muscle material.

In isotropic material the mechanical properties are independent of the orientation in the material. Biological material, like connective tissues and muscles shows a specific directionality of substructures which makes the material to behave anisotropically. Nevertheless, for the sake of simplicity of mathematical analysis, commonly the material is assumed to behave isotropically. Under this condition the myocardial material is supposed to be prestretched in the fiber direction. During unloaded contraction of myocardial material $\left(l_{s}=1.6 \mathrm{~km}\right)$ there is no stress $\left.{ }^{41}\right)$ and this situation is taken to define zero strain. In the passive state, when no activation is present, the material is supposed to be soft, and hence the stress to be minor. During the active state the Young's-modulus is assumed to increase, which results in a large strain dependent stress. The principal natural strains $\left(\varepsilon_{1}, \varepsilon_{2}\right.$ and $\left.\varepsilon_{3}\right)$ are defined as 


$$
\begin{aligned}
& \varepsilon_{1}=\ln \left(\frac{l_{1}}{L_{1}}\right) \\
& \varepsilon_{2}=\ln \left(\frac{l_{2}}{L_{2}}\right) \\
& \varepsilon_{3}=\ln \left(\frac{l_{3}}{L_{3}}\right)
\end{aligned}
$$

where $1_{1}, 1_{2}, 1_{3}$ describe a volume element of myocardial material in a given condition of strain (fig. 5.16). $L_{1}, L_{2}, L_{3}$ describe the same element in a state of zero strain.

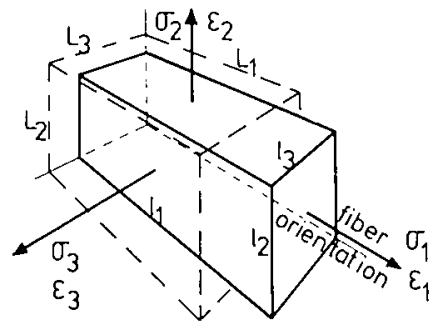

Figure 5.16 .

Deformation of a volume element of myocardial material. The initial dimension of the cube are denoted by $L_{1}, L_{2}$ and $L_{3}$. After deformation these dimensions are $l_{1}, l_{2}$ and $l_{3}$. The components of stress and strain in the principal directions are indicated.

The strains cause a change of shape of the volume element as well as a change in volume. It can be shown that the latter is controlled by the quantity

$$
\varepsilon_{m}=\frac{\varepsilon_{1}+\varepsilon_{2}+\varepsilon_{3}}{3}
$$

where $\varepsilon_{m}=$ mean natural strain.

Consequently the real deformation is due to the deviatoric principal strains $\left(\varepsilon_{\mathrm{d} 1}, \varepsilon_{\mathrm{d} 2}, \varepsilon_{\mathrm{d} 3}\right)$. It holds

$$
\varepsilon_{\mathrm{dl}}=\varepsilon_{1}-\varepsilon_{\mathrm{rl}}
$$




$$
\begin{aligned}
& s_{d 2}=\varepsilon_{2}-\varepsilon_{m} \\
& s_{d}=\varepsilon_{3}-\varepsilon_{m}
\end{aligned}
$$

It should be remarked that the definition of principal natural strains in relations (5.5.1) only has physical sense if the path of stress is straight, which means, that the ratio of principal stresses is constant during deformation. In this case a quantity of state, the effective strain (E) can be defined as

$$
\bar{\varepsilon}=\sqrt{\frac{2}{3}\left(\varepsilon_{d l}^{2}+\varepsilon_{d 2}^{2}+\varepsilon_{d 3}^{2}\right)}
$$

The principle stresses corresponding to a given state of strain are $\sigma_{1}, \sigma_{2}$ and $\mathrm{o}_{3}$. The average stress or hydrostatic stress $\left(\sigma_{\mathrm{m}}\right)$ being

$$
\sigma_{\mathrm{mII}}=\frac{\sigma_{1}+\sigma_{2}+\sigma_{3}}{3}
$$

controls the change in volume through the compressibility of the material. Another quantity of state in terms of stress can be defined as the effective stress

$$
\bar{\sigma}=\sqrt{\frac{3}{2}\left(\sigma_{d l}{ }^{2}+\sigma_{d 2}{ }^{2}+\sigma_{d 3}{ }^{2}\right)}
$$

where

$$
\begin{aligned}
& \sigma_{\mathrm{d} 1}=\sigma_{1}-\sigma_{\mathrm{m}} \\
& \sigma_{\mathrm{d} 2}=\sigma_{2}-\sigma_{\mathrm{m}} \\
& \sigma_{\mathrm{d} 3}=\sigma_{3}-\sigma_{\mathrm{ml}}
\end{aligned}
$$

are the deviatoric principle stresses.

In the case of a straight path of stress it can be shown that

$$
\varepsilon_{\mathrm{d} 1}=\frac{3}{2} \frac{\bar{\varepsilon}}{\bar{\sigma}} \sigma_{\mathrm{d} 1}
$$




$$
\begin{aligned}
& \varepsilon_{\mathrm{d} 2}=\frac{3}{2} \frac{\bar{\varepsilon}}{\bar{\sigma}} \sigma_{\mathrm{d} 2} \\
& \varepsilon_{\mathrm{d} 3}=\frac{3}{2} \frac{\bar{\varepsilon}}{\bar{\sigma}} \sigma_{\mathrm{d} 3}
\end{aligned}
$$

where the factor $\bar{\varepsilon} / \bar{\sigma}$ depends on the mechanical properties of the material, and commonly is detemined by performing a tensile test. In a tensile test it holds that $\sigma_{2}=\sigma_{3}=0$ and hence, as readily can be derived, it follows that

$$
\sigma_{1}=\bar{\sigma}
$$

Since muscle material is virtually incompressible ${ }^{18)}$ it holds that $\varepsilon_{m}=0$. Thus in case of a tensile test it follows that

$$
\varepsilon_{2}=\varepsilon_{3}=-\frac{1}{2} \varepsilon_{1}
$$

which results in

$$
\varepsilon_{1}=\bar{\varepsilon}
$$

Thus it is clear that the relation between stress and strain as obtained from a tensile test is representative for the proportionality factor in equations (5.5.8). In in vitro experiments on cardiac muscle, as described in section 5.4, the conditions of a tensile test are present.

The assumption of negligible compressibility is supported by the fact that the wave velocity of ultrasound in cardiac muscle is approximately $5 \%$ larger than in water 51 ), which implies that for high frequencies of compression ( $~$ $1 \mathrm{MHz}$ ) the compressibility of muscle is approximately $10 \%$ less than water.

\subsection{Anisotropic myocardial material}

In this section it will be shown, that the necessary additional relations between the stress and strain components also can be obtained by the assumption of anisotropy of the cardiac muscle material. As could be expected, these relations appear to be different from those in the case of isotropic cardiac properties, as analized in section 5.5 .

In general it is a major problem to account for anisotropy in the analysis of large deformations. Technically the problem can approximately be solved by 
introducing anisotropy factors, but the number of paraneters to deal with increases, wicil complicates the analysis. As to the present situation it should be renarked that very little information about anisotropic properties of myocardial material is available. However, it seems reasonable to describe the myocardial tissue as a contractile fiber structure embedded in a soft incompressible material (section 5.7). In this model the tissue is able to bear only longitudinal tensile stress as exerted by the muscle fibers. Transversal displacements of inuscle fibers with respect to each other occur easily, as demonstrated by Hort ${ }^{2 l}$ ). In a ilicroscopic observation of a frozen sample of myocardium he counted the number of intersected muscle fibers, when moving in a straight line from the epicardiulil to the endocardium. A larger number of $f i$ bers was found when the myocardium was frozen at end-systole than when frozen at end-diastole. The number of intersected fibers appeared to be proportional to the wall thickness wich implies rearrangement of the fibers during deformation of the myocardium. This suggests that the influence of the cross-connections between the fihers on the mechanical properties of cardiac muscle can be neglected. For this reason these cross-connections are not considered in the concept of anisotropic cardiac material.

If the material which surrounds the fibers has an isostatic pressure $-p$, and the fibers generate the stress $o_{f}\left(\varepsilon_{l}\right)$, the state of stress is given by

$$
\begin{aligned}
& \sigma_{1}=-p+o_{f}\left(\varepsilon_{1}\right) \\
& \sigma_{2}=-p \\
& \sigma_{3}=-p
\end{aligned}
$$

Fron the very definitions of effective stress and effective strain it can now be concluded that neither of these quantities is affected by a superimposed isostatic pressure. Hence the constituent equation $\bar{\sigma}=\bar{c}(\bar{\varepsilon})$ is independent of any isostatic pressure, exposed to the material. Thus when having determined $\sigma_{1}=o_{1}\left(\varepsilon_{1}\right)$ in a tensile test, the result is representative of $\sigma_{f}\left(\varepsilon_{1}\right)$ in relation (5.6.1). Anisotropy is introduced by assuming that in the 1-direction the material behaves according to relation 5.6 .1 , while in the 2 - and 3 -directions the material behaves ideally plastic. This means that the stress in these directions is independent of the strain. However, the condition of constancy of volume requires that the sum of strains in the 2 - and 3 -direction is opposite to the strain in the 1-direction, thus satisfying 


$$
\varepsilon_{1}+\varepsilon_{2}+\varepsilon_{3}=0
$$

In the model of the left ventricular dynamics the intramyocardial pressure is defined as the pressure in the fluidlike material surrounding the muscie fiber, which is denoted by the symbol $p$ in relation (5.6.1).

5.7. Influence of isotropy or anisotropy of myocardial material on the systolic pressure-volume relation of the left ventricle

Since the mathematical description of the model of the left ventricle depends on the properties of the myocardial material, it has to be decided, whether heartmuscle should be treated as isotropic or anisotropic material. It is likely, for instance, that isotropy leads to a stiffer behavior of the left ventricle because of the energy storage due to deformation of the muscle cross-section. To evaluate the characteristics of the myocardium, left ventricular systolic capacitance $\left(C_{1 V}\right)$ as computed for isotropic as well as for anisotropic material is compared with this capacitance as determined in animal experiments ${ }^{48)}$ (chapter 11). The left ventricular systolic capacitance is defined as the slope of the function relating end-diastolic volume to maximum generated left ventricular pressure during isovolumic contraction. In this section the left ventricle is simulated by a thin-walled cylinder. The wallmaterial is considered to be non linearly elastic and incompressible. Thus in a given state of strain the modulus of elasticity is given by

$$
H=\frac{d \bar{\sigma}}{d \dot{\bar{E}}}
$$

The assumption of purely elastic, non-viscous behavior is reasonable, because during an isovolumic contraction the velocity of sarcomere shortening is so low that its influence on the tensile muscle stress is negligible. In the case of isotropic muscle properties the following relation was found for the capacitance (see Appendix 5.1):

$$
C_{\text {IV }}^{i s} \simeq \frac{3}{2} \frac{V_{c} r}{H h}
$$

where $r=$ average value of inner and outer radius of the cylinder

$v_{c}=$ volume of the cylinder with radius $r$

$\mathrm{h}=$ wall-thickness 


\section{$H=$ modulus of elasticity, defined by relation (5.7.1)}

In the case of anisotropic muscle material the following relation was found for the capacitance (see Appendix 5.2):

$$
C_{l v}^{a n}=\frac{9 v_{c}^{r}}{2} \frac{b h}{b h}
$$

which value is 3 times $C_{\text {lv }}^{\text {is }}$ :

In a nomal heart during diastole the ratio $r / h: 2.5$ (chapter 9 ). Applying this ratio to experiments of Suga et al. ${ }^{48}$ ) yields $V_{C} \simeq 30 \mathrm{ml}$. From experiments of pollack et al. ${ }^{41}$ ) on isosarcometric contractions of cardiac muscle the modulus of elasticity $(H)$ of activated heart muscle is computed to be $3.8 \cdot 10^{5} \mathrm{~N} \mathrm{~m}^{-2}$. Substituting these values into the relations (5.7.2) and (5.7.3) results in

$$
C_{7 v}^{i s}=3.0 \cdot 10^{-10} \mathrm{~N}^{-1} \mathrm{nt}^{5}
$$

and

$$
C_{\mathrm{TV}}^{\text {an }}=8.9 \cdot 10^{-10} \mathrm{~N}^{-1} 5
$$

Application of $r / h=2.5$ and $V_{C}=30 \mathrm{~m} 1$ to the experiments of suga et al. ${ }^{48}$ ) gives $C_{7 v}=8.7 \cdot 10^{-10} \mathrm{~N}^{-1} \mathrm{~m}^{5}$, which is close to the value calculated for anisotropic muscle material (5.7.5). Therefore, the concept of anisotropy is considered to be more realistic than the one of isotropy. Moreover, the computer simulation based upon anisotropy agreed fairly well with the situation in animal experiments, at least as far as left ventricular pressure and aortic volume flow are concerned, a computer simulation based upon isotropy did not agree with the experimental situation (chapter 11). 


\section{Literature}

1. Binkhorst, R.A., Hoofd, L., Vissers, A.C.A.: Temperature and force-velocity relationship of human muscies. J Appl Physiol 42, 471 - 475 (1977).

2. Brady, A.J.: A measurement of the active state in heart muscle. Cardiovasc Res Suppl. 1, $11-17$ (1971).

3. Brutsaert, D.L., Claes, V.A.: Onset of mechanical activation of mammalian heart muscle in calcium- and strontium-containinn solutions. Circ Res 35, $345-357(1974)$.

4. Brutsaert, D.L., Sonnenblick, E.H.: Nature of the force-velocity relation in heart muscle. Cardiovasc Res Suppl. 1, 18 - 33 (1971).

5. Brutsaert, D.L., Claes, V.A., Sonnenblick, E.H.: Velocity of shortening of unloaded heart muscle and the length-tension relation. Circ Res 29, 63-75 (1971).

6. Brutsaert, D.L., Henderson, A.H.: Time course of mechanical activation in cardiac muscle. Eur J Cardiol 1, 201 - 208 (1973).

7. Cleworth, D.R., Edman, K.A.P.: Changes in sarcomere length during isometric tension development in frog skeletal muscle. J Physiol (London) 227, 1- 17 (1972).

8. Cooper, S., Eccles, J.C.: Isometric responses. J Physiol 69, $379-385$ (1930).

9. Edman, K.A.P., Nilsson, E.: The mechanical parameters of myocardial contraction studied at a constant length of the contractile element. Acta Physiol Scand 27, $205-219$ (1968).

10. Edman, K.A.P., Nilsson, E.: The dynamics of the inotropic change pruduced by altered pacing of rabbit papillary muscle. Acta Physiol Scand 76, 236 247 (1969).

11. Fabiato, A., Fabiato, F.: Dependence of calcium release, tension generation and restoring forces on sarcomere length in skinned cardiac cells. Eur $\mathrm{J}$ Cardiol 4, 13 - 27 (1976).

12. Fung, Y.C.: Comparison of different models of the heart muscle. J Biomech 4. $289-295(1971)$.

13. Fung, Y.C.: Mathematical representation of the mechanical properties of the heart muscle. J Biomech 3 , 381 - 404 (1970).

14. Gibbs, C.L., Chapman, J.B.: Effects of stimulus conditions, temperature, and length on energy output of frog and toad sartorius. Am J Physiol 227, 964 - 971 (1974).

15. Gordon, A.M., Huxley, A.F., Julian, F.J.: The relation between sarcomere length and characteristics of isometric twitch contractions of frog sartorious muscle. J Physiol (London) 184, 170 - 192 (1966). 
16. Henderson, A.H., Ocken, E. van, Brutsaert, D.L..: A reappraisal of forcevelocity measurements in isolated heart muscle preparations. Eur J Cardiol $1,105-118(1973)$.

17. Henderson, A.H., Catte]1, H.R.: Length-induced changes in activation during contraction. A study of mechanical oscillations in strontium-mediated contractions of cat and frog heart muscle. Circ Res 38, $289-296$ (1976).

18. Hill, A.V.: A discussion on muscular contraction and relaxation: their physical and chemical basis. Proc Roy Soc Lond (Biol) 137, 40 - 87 (1950).

19. Hill, A.V.: The heat of shortening and the dynamic constants in muscle. Proc Roy Soc Lond (Biol) 126, 136 - 165 (1938).

20. Hodgkin A.L., Horowics, P.: Potassium contractures in single muscle fibres. J Physiol 153, 386 - 403 (1960).

21. Hort, W.: Makroskopische und mikrometrische Untersuchungen a!n Hyocard verschieden stark gefillter linker Kammern. Virchows Arch (Pathol Anat) $333,523-564(1960)$.

22. Huxley, A.F., Niedergerke, R.: Structural changes in muscle during contraction. Nature 173, $971-973$ (1954).

23. Huxley, A.F., Simons, R.ll.: Proposed mechanism at force generation in striated muscle. Nature 233, 533 - 538 (1971).

24. Iwazumi, T.: A new field theory of muscle contraction. Dissertation University of Pennsylvania (1970).

25. Janz, R.F., Grimm, A.F.: Deformation of the diastolic left ventricles: I. Nonlineair elastic effects. Biophys J 13, $689-704$ (1973).

26. Julian, F.J.: Activation in a skeletal muscle contraction model with a modification for insect fibrillar muscle. Biophys J $9,547-569$ (1969).

27. Julian, F.J., Sollins, M.R.: Sarcomere length tension relations in Tiving rat papillary muscle. Circ Res 37, 299 - 308 (1975).

28. Julian, F.J., Sollins, M.R., Moss, R.L.: Absence of a plateau in the length-tension relationship of rabbit papillary muscle, when internal shortening is prevented. Nature 260, 340 - 342 (1976).

29. Kaufmann, R.L., Lab, M.J., Hennekes, R., Krause, H.: Feedback interaction of mechanical and electrical events in the isolated mammalian ventricular myocardium (cat papillary muscle). Pflüger Arch 324, $100-123$ (1971).

30. Krueger, J.W., Pollack, G.M.: Myocardial sarcomere dynamics during isometric contraction. J Physiol (London) 251, 627 - 643 (1975).

31. Loeffler, L., Sagawa, K.: A one-dimensional visco-elastic model of cat heart muscle studies by small length perturbations during isometric contractions. Circ Res 36, 498 - 512 (1975).

32. Meiss, R.A., Sonnenblick, E.H.: Controlled shortening in hart muscle: velocity-force and active-state properties. Am J Physiol 222, 630-639 (1972). 
33. Nachmansohn, D.: The neuro-muscular junction - The role of acetylcholine in exitable membranes. In: G.H. Bourne, ed.: The structure and functionof muscle. vol. III, New York: Academic (1972).

34. Noble, M., Pollack, G.M.: Molecular mechanics of contraction. Circ Res 40 , $333-342(1977)$.

35. Noble,M.I.M.: The diastolic viscous properties of cat papillary muscle. Circ Res 40, $288-292$ (1977).

36. Noble, M. I.: Problems in the definition of contractility in terms of myocardial mechanics. Eur J Cardiol 1, 209 - 216 (1973).

37. Page, E., Fozzard, H.A.: Capacitive, resistive and syncytial properties of heart muscle - ultrastructural and physiological considerations.

In: G.H. Bourne, ed.: The structure and function of muscle. vol. II, 2nd ed. New York: Academic (1972).

38. Parmley, W.W., Chuck, L., Chatterjee, K., Swan, H.J.C., Klausner, S., Glantz, S.A., Ratshin, R.: Acute changes in the diastolic pressure-volume relationship. Eur J Cardiol (Suppl) 4, 105 - 120 (1976).

39. Parmley, W.W., Chuck, L.: Contractile length-dependent changes in myocardial state. Am J Physiol 224, 1195 - 1199 (1973).

40. Paulus, W.J., Claes, V.A., Brutsaert, D.L.: Physiological loading of isolated mammalian cardiac muscle. Circ Res 39, 42 - 53 (1976).

41. Pollack, G.H., Krueger, J.W.: Sarcomere dynamics in intact cardiac muscle. Eur J Cardiol 4, 53 - 65 (1976).

42. Pollack, G.H., Huntsman, L.L., Verdugo, P.: Overextension of series elasticity. Circ Res $31,569-579$ (1972).

43. Ross, J., Covell, J.W., Sonnenblick, E.H., Braunwald, E.: Contractile state of the heart characterised by force-velocity relations in variably afterloaded and isovolumic beats. Circ Res 18, $149-163$ (1966).

44. Sonnenblick, E.H., Spiro, D., Spotnitz, H.M.: The ultrastructural basis of Starling's law of the heart. The role of the sarcomere in determining ventricular size and stroke volume. Am Heart J $\underline{68}, 336-346$ (1964).

45. Sonnenblick, E.H., Spiro, D., Cottrel1, T.S.: Fine structural changes in heart muscle in relation to the length tension curve. Physiology 49 , $193-200$ (1963).

46. Spiro, D., Sonnenblick, E.H.: Comparison of the ultrastructural basis of the contractile process in heart and skeletal muscle. Circ Res 15 (suppl. 2) $14-3^{\circ}(1964)$.

47. Spotnitz, H.M., Sonnenblick, E.H., Spiro, D.: Relation of ultrastructure to function in intact heart. Sarcomere structure relative to pressurevolume curves of intact left ventricles of dog and cat. Circ Res 18, 49 - $66(1966)$. 
48. Suga, H., Yamakoski, K.: Left ventricle as a compression pump. Eur J Cardiol 4, $97-103(1976)$.

49. Taylor, R.R.: Active length-tension relations compared in isometric, afterloaded and isotonic contractions of cat papillary muscle. Circ Res 26, $279-287$ (1970).

50. Tirosh, R., Liron, N., Oplatka, A.: A hydrodynamic model for muscle contraction. Biorheology 12, 99 (1975).

51. Welis, P.N.T.: Physical principles of ultrasonic diagnosis. Academic Press London, New York (p. 6) (1969).

52. Winegrad, S.: Resting sarcomere length tension relation in living frog heart. J Gen Physiol 64, 343 - 355 (1974). 


\section{CHARACTERISTICS OF THE CORONARY CIRCULATION}

\subsection{Introduction}

In the model the coronary circulation is presumed to consist of several compartments, i.e.:

- The large coronary arteries. This part includes the epicardial arteries and their branches with an inner diameter $>400 \mathrm{\mu m}$. The side-branches of the epicardial arteries enter the myocardium perpendicular to the epicardial arteries and continue their course down to the subendocardial layers.

- The microcirculation. This part consists of three subcompartments, (1) the arterioles and smaller arteries (2) the capillaries and (3) the venules and part of the veins.

- The right atrium and the large coronary veins.

Since hemodynamics vary from layer to layer, in the model each subcylinder has its own microcirculation. Blood flow into the capillaries is regulated by vasoactivity of the arterioles. The intramyocardial pressure, defined as the pressure in the soft material surrounding the myocardial fibers and the microcirculatory vessels, compresses this part of the coronary circulation which results in a decrease in bloodvolume and increase in blood flow resistance.

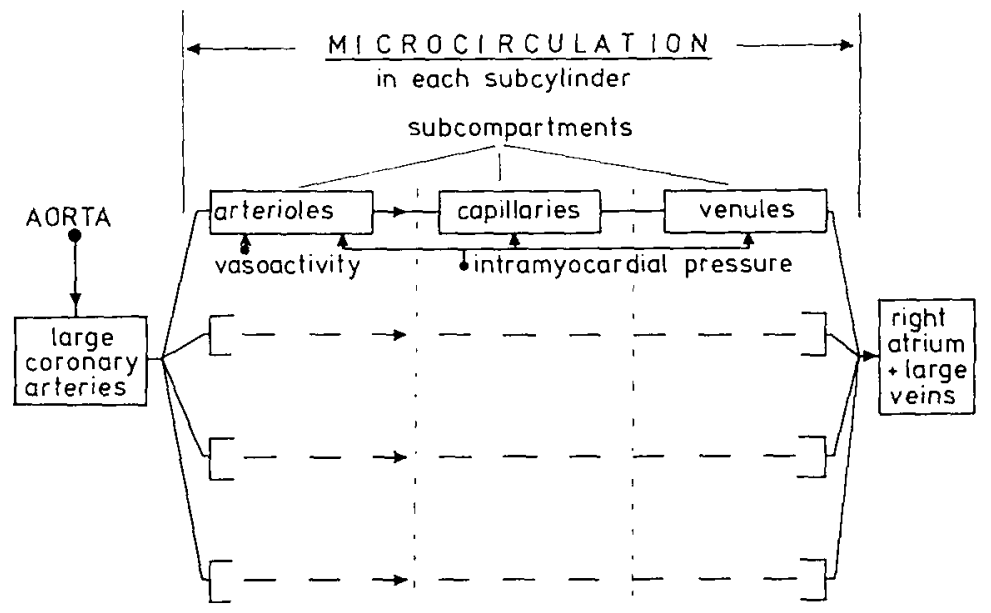

Figure 6.1.

Block diagram of the coronary circulation. 
The blood from the venular compartment of the various subcylinders is presumed to be collected in the right atrium. In fig. 6.1 a block-diagram of the various compartments is given.

In section 6.2 the physical properties of the large coronary arteries (I.D. $>400 \mu, m)$ are analyzed on the basis of animal experinents in which the pulse wave front velocity in and dimensions of these arteries are determined. In section 6.3 a model of the coronary microcirculation is described. Section 6.4 deals with the mathematical representation of the coronary circulation. In appendices several mathematical derivations are presented, which are related to the hemodynamics of the large coronary arteries.

\section{Physical properties of the large coronary arteries in dogs}

\subsection{Introduction}

The model of the large coronary arteries is assumed to consist of an inertance, two equal resistances and a capacitance, together characterising the impedance of those arteries (fig. 3.3). These arteries are assumed not to be influenced directly by the cardiac contraction.

Data on the inertance of the large coronary arteries could not be obtained from the literature, and the resistance of these vessels has not been investigated systematically. An estinate of the resistance can be obtained from flow and pressure measurements as presented in literature ${ }^{19}$ ). The static capacitance of the large coronary arteries was determined by Patel et al. ${ }^{16}$ ). The presence of a dynamic capacitance in the coronary arteries was demonstrated by Lewi et al. ${ }^{12)}$, but these investigators did not quantify this capacitance. Absolute values of the dynamic capacitance were given by Douglas et al. ${ }^{6}$ ). In their study, however, the magnitude of the dynamic capacitance was underestimated, which is mainly due to the substitution of too high pressure and time differences in the differential equation which partly describes their capacitance-resistance model.

The data available on the volume of the epicardial coronary arteries were derived from in vitro studies ${ }^{6}$ ). These data probably are different from those valid for the in vivo situation.

The present study on the epicardial coronary arteries was conducted to determine their impedance, representative of the in vivo situation. The length and diameter of the coronary arteries and their branches were determined from araldite casts and from this geometry the resistance, volume and inertance of 
the arteries were calculated. In these calculations corrections were made for differences in the geometry of the vessels in the in vitro and in vivo situation. The correction factor for the differences in diameters of the coronary arteries in the in vivo and in vitro situation is obtained from determination of the internal diameter of comparable segments of the LADC in both situations. The wave front velocity, which is the velocity of transmission of a pressure change in a vessel, was determined in in vivo experiments. The capacitance of the epicardial coronary arteries was determined from the wave front velocity in vivo, making use of the data obtained from the casts. A preliminary abstract of this study has been published ${ }^{1)}$.

\subsubsection{Methods}

In vitro experiments (casts)

The experiments were performed on 9 mongrel dogs of varying weight, unknown age and of either sex. Under anaesthesia the animals were killed by bleeding and the hearts were removed and weighed. The aortic valves were closed by suturing. Araldite (F type $H Y$ 943) was injected into the aorta at a pressure of $100 \mathrm{mmHg}$ in four hearts and $190 \mathrm{mmHg}$ in five other hearts to fi11 up the coronary arteries. After 2 hours of hardening at $35^{\circ} \mathrm{C}$ the hearts were submerged in a $20 \%$ solution of potassium hydroxyde to dissolve the tissues, whereupon the casts were rinsed and dried.

Each cast was devided in three trees (fig. 6.2), corresponding to the perfusion areas of the right coronary artery (RC) and the anterior descending branch (LADC) and the circunflex branch (LCC) of the left coronary artery, respectively. The septal branch was arbitrarily considered to be part of the LADC. All branches smaller than $0.4 \mathrm{~mm}$ in diameter were removed. The length and diameter of the remaining branches in relation to their position were measured with a vernier callipers.

\section{Experimental set-up in vivo experiments (fig. 6.3)}

The experiments were performed on 10 mongrel dogs of either sex, unknown age, and ranging in weight from $28-45 \mathrm{~kg}$. The animals were premedicated with Hypnorm $^{(\mathrm{x})}\left(1 \mathrm{ml} / \mathrm{kg}\right.$ body weight) as described by Marsboom et al. ${ }^{13}$ ). Anaesthesia was induced with sodium pentobarbital $(10 \mathrm{mg} / \mathrm{kg}$ body weigth I.V.) and after endotracheal intubation, was maintained by oxygen-nitrous oxide. Ven-

x) $1 \mathrm{ml}$ Hypnorm contains $10 \mathrm{mg}$ fluanison and $0.2 \mathrm{mg}$ fentanyl base. 

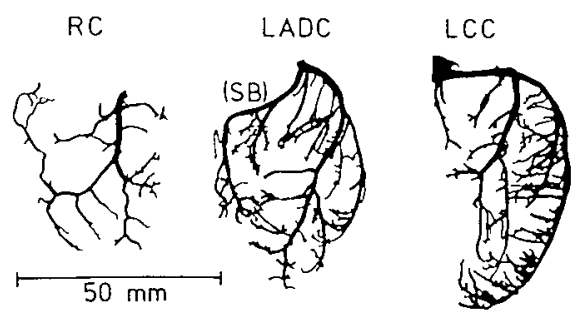

Figure 6.2 .

Araldite casts of the vascular trees of the right coronary artery (RC), and the anterior descending (LADC) and circumflex (LCC) branches of the left coronary artery. The septal branch (SB) is considered to be part of the LADC.

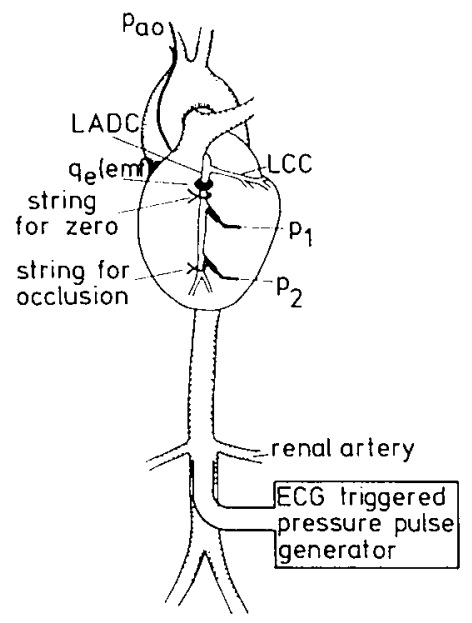

Figure 6.3.

Experimental set-up for the in vivo measurements. $p_{1}$ and $p_{2}$ are devices for pressure measurement as shown in fig. $6.4 . q_{e}$ is an electromagnetic flowmeter.

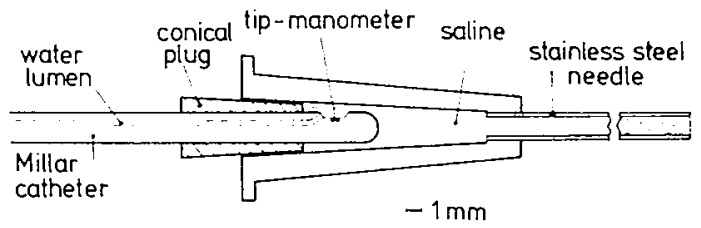

Figure 6.4.

Device for pressure measurements in the coronary arteries. The stainless steel needle is inserted into the side branches of the $L A D C$. 
tilation was kept constant during the experiment with a positive pressure respirator (Bird). The ECG was derived from the limb leads. Ascending aortic pressure was measured through the left carotid artery with a polyethylene catheter connected to a pressure transducer (Telco RA 9). The chest was opened through the left fifth intercostal space and the heart suspended in a pericardial cradle.

The coronary artery pressure was measured in the first two major side branches of the I.ADC with short $(25 \mathrm{~mm})$ leveled stainless steel needles (I.D. $0.95 \mathrm{~mm})$. The tip of each needle was positioned by palpation at the origin of the branch, carefully avoiding protrusion of the needle into the lumen of the LADC. Millar (PC 470) catheter tip micromanometers were plugged into the connector cones of both needles ( $\mathrm{fig} .6 .4$ ). The advantage of this system is the rather high frequency response $(100 \mathrm{~Hz},-3 \mathrm{~dB})$ and the absence of artefacts due to movements of the catheter. The difference between the pressure pulses at the proximal and at the distal site was obtained from electrical subtraction after careful adjustment of both pressure calibrations 21 ) and was recorded continuously, as described by Spencer 22 ).

An electromagnetic flowprobe was placed on the LADC proxinal to the sites of pressure measurenent. The probe was connected to a sine-wave electromagnetic flowineter with a carrier frequency of $600 \mathrm{~Hz}$ (Transflow 600). The upper frequency response of the flometer was $100 \mathrm{~Hz}(-3 \mathrm{~dB})$. Mean flow was obtained by an active integrating network. The determined variables were recorded on a Schwarzer 10-channel physiological recorder (frequency response: $150 \mathrm{~Hz},-3 \mathrm{~dB}$; speed $200 \mathrm{~mm} / \mathrm{sec}$ ) and on a Hewlett Packard (3521B) electronagnetic tape recorder (speed $15 \mathrm{inch} / \mathrm{sec}$ ). The pressure pulses were also recorded on an oscilloscope (Tektronix, type $R$ 5031) and photographed with a polaroid camera (Tektronix, type $C 50$ ).

An ECG triggered pressure puise generator (Dreyssen) was connected to the abdominal aorta through a PVC tube with an inner diameter of $9 \mathrm{~mm}$. The tube was placed about halfway between the renal arteries and the iliac bifurcation. For a precise determination of the delay between both of the recorded coronary artery pressures, the ratio of the rise time of the pressure pulse and the delay time must be as small as possible. A compromise was found in a pressure rise time of $10 \mathrm{msec}$ and a minimum distance between the sites of pressure measurement of $20 \mathrm{~mm}$. Shorter rise times could not easily be generated. Moreover, a pressure signal with a shorter rise time than 10 ins contains important frequency components over $30 \mathrm{~Hz}$. It is possible that such high frequencies in- 
troduce errors in the determination of the wave front velocity, due to changed elastic properties of the artery at these frequencies. The distance between the sites of pressure measurement was the distance between the first large side branches of the $L A D C$, which seldom exceeds $30 \mathrm{~mm}$.

After installation of the measuring devices and the pressure generator, the latter was filled by slowly suction of $200 \mathrm{ml}$ of blood from the aorta. The delay between the ECG and the pump was adjusted in such a way that the pressure pulse appeared in the coronary system early in diastole. An adequate pressure pulse could be generated by rapid injection of $20 \mathrm{ml}$ of blood into the aorta. The arrival of the pressure pulse in the coronary system was recorded by the pressure sensing devices in the side branches of the LADC. The wave front velocity $\left(v_{w f}\right)$ in the LADC is defined as the ratio of the distance between the sites of pressure measurement and the time interval between the change in pressure at the proximal and at the distal site of measurement. The signals were compared at $25 \%$ of the maximum pressure change (fig. 6.7 a). At higher levels than $25 \%$ of the maximum pressure change, irrigularities in the slope of the pressure signal are more pronounced. This phenomenon may be partly due to reflections of the pressure wave in the aorta at the aortic valve.

Under control conditions several series of approximately 10 pulses were generated while the variables were recorded continuously. After each series the pump was refilled. After the control series in all experiments a coronary vasodilator, dipyridamole $(5 \mathrm{mg} / \mathrm{kg})$, was administered intravenously. After the flow in the $L A D C$ had reached a higher stationary level, a similar series of pulses was generated as in the control situation. To induce reflections the LAOC was temporarily occluded by a string distal to the sites of pressure measurement and several series of pulses were generated.

At the end of each experiment the LADC was occluded distal to the proximal pressure device, which was replaced by a graduated syringe to calibrate the electromagnetic flow probe. This method of calibration was previously described for the femoral artery ${ }^{18)}$. After the experiments the animals were killed with $\mathrm{KCl}$ and the hearts were removed and weighed.

The relation between the vessel diameter proximal and distal to a bifurcation

Since the method used for the determination of the wave front velocity only applies for a system free of reflection it is important to know the magnitude of the reflections in the coronary system. As to this, we studied the relation between the vessel diameters proximal and distal to a bifurcation. In 
theory in a symmetric bifurcation (fig. 6.5) the magnitude of the reflection is minimum for a ratio of the diameters of the proximal branch to the distal branches of 1.31 (appendix 6.1). Simular results were presented by McDonald ${ }^{15}$ ).

Figure 6.5.

Schenlatic representation of a coronary artery bifurcation

rprox $=$ radius of the proximal artery

rdist $=$ radius of the distal branches

Nprox $=$ number of distal branches of $0.4 \mathrm{~mm}$ diameter of the proximal branch

$N_{\text {dist }}=$ number of distal branches of $0.4 \mathrm{~mm}$ diameter of a distal branch

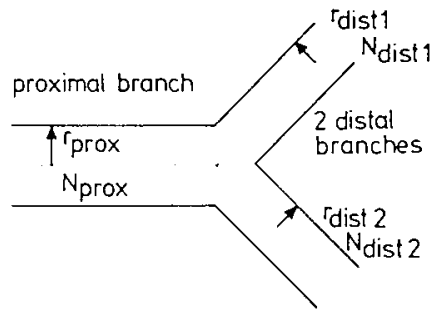

To study this ratio in the coronary vasculature, the following definition is used. The number $(N)$, which is a characteristic number for a coronary artery branch, is defined as the number of branches of $0.4 \mathrm{~min}$ in diameter, which are distal to that coronary artery branch. In 12 trees of the araldite casts (4 LADC, 4 LCC, 4 RCC) for all branches the number (N) of distal branches with a diameter of 0.4 min was determined. For each tree the branches were grouped according to their diameter, starting at a diameter of $0.4 \mathrm{~mm}$ and increasing in steps of $0.1 \mathrm{~mm}$ up to the largest branch diameter of the tree. In each group the average of $\mathrm{N}$ was determined. The data of the 12 trees were pooled and next the best fitting regression was calculated.

In a symimetric bifurcation $N$ belonging to the proximal branch is two times $N$ belonging to one distal branch. Applying this to the experimentally determined relation between $\mathrm{N}$ and the vessel diameter, the ratio of proximal to distal branch diameters can be calculated (see results in this section).

Determination of the cross-sectional stiffness $K$ of the epicardial coronary arteries

The cross-sectional stiffness $K$ of a vessel is defined as the ratio of increase in pressure and increase in cross-sectional area, normalized on crosssectional area. The value of $k$ was determined from

$$
k \simeq 1.25 \rho v_{\mathrm{wf}}^{2}
$$


where $\rho=$ density of blood

$v_{\mathrm{wf}}=$ experimentally determined wave front velocity in a proximal segment of the LADC.

This relation is the result of a computer simulation, which is based on the equations for waves in arteries, as presented by Womersley 15,24) (appendix 6.2). In the in vivo experiments the wave front velocity in the LADC was measured using a pressure step with a rise time of approximately $10 \mathrm{~ms}$, in which case the pressure signal contains frequencies up to $32 \mathrm{~Hz}$. Thus, the elastic behavior of the wall of the vessel segment under investigation is assumed to be constant in the frequency range $5-32 \mathrm{~Hz}$. This is supported by the finding that this behavior of systemic arteries was found not to vary significantly at frequencies over $5 \mathrm{~Hz}^{4}$ ). Frequencies lower than $5 \mathrm{~Hz}$ are not relevant since the method to determine the moment of the pressure increase is insensitive to low frequencies.

Determination of the in vivo diameter of the LADC

To get information on the relation between the volumes of the coronary arteries in vivo and in vitro, the diameter calculated from the casts was compared to the diameter as determined in vivo for a corresponding part of the LADC. In ivo the diameter of the coronary artery segment between the sites of pressure measurement $p_{1}$ and $p_{2}$ was calculated from the fluid resistance according to Poiseuille's law.

$$
\Delta p=p_{1}-p_{2}=\frac{8 \eta 1}{\pi r^{4}} q
$$

where $\Delta p=$ pressure difference over the vessel segment

$\eta=$ viscosity of blood

1 = length of the vessel length

$r=$ radius of the vessel segment

$q_{e}=$ volume flow into the vessel segment

Relation 6.2 .2 requires stationary coronary artery flow. During the end of diastole coronary artery flow is stationary since coronary artery volume flow as well as the pressure difference is approximately constant. Moreover, the time constant, belonging to the fluid impedance of the vessel segment, which is determined by the resistance and inertance of the vessel segment, is approximately $40 \mathrm{~ms}$. The duration of the diastole is much larger than this 40 
ms and, therefore, in the end-diastolic phase the influence of changes in coronary volume flow, due to the transition of systole to diastole, can be neglected. It should be remarked that the pressure difference $\Delta p$ is very small. For this reason is was measured at two different flow levels to eliminate the influences of the drift of the pressure transducers. However, equation (6.2.2) in this simple form does not apply for the real situation since the small side branches cause some flow leakage. lloreover, tapering of the vessels influences the pressure difference. Also the radius of the vessel and hence the resistance depend on internal pressure, and since $\Delta p$ was determined at two different flow levels, the internal pressure levels are different in both cases. The correction for the latter two effects are derived in appendix 6.3. The following relation was found for the radius $r_{d}$ during diastole:

$$
r_{d}^{4}=\frac{q_{d}-q_{s} \cdot\left(\frac{1+p_{d} / k}{1+p_{s} / k}\right)^{2}}{\Delta p_{d}-\Delta p_{s}} \cdot \frac{1-(1-\lambda)^{0.431}}{0.431 \lambda} \cdot \frac{8 n 1}{\pi}
$$

where $q_{d}, q_{s}=$ diastolic and systolic volume flow into the LADC

$p_{d}, p_{s}=$ diastolic and systolic pressure in the LADC

$\Delta p_{d}, \Delta p_{s}=$ diastolic and systolic pressure difference over the vessel segment

$K=$ cross-sectional stiffness of the vessel segment

$\lambda \quad=$ fraction of flow leakage from the vessel segment.

The leakage fraction $\lambda$ is defined as the ratio of volume flow leakage through the side branches of the vessel segment and the volume flow at the entrance of the vessel segment. During vasodilatation, caused by dipyridamole, the perfusion rate is assumed to be maximum in all parts of the heart muscle. Under these circumstances, the leakage fraction $\lambda$ was determined and expressed as the ratio of flow into the LADC with and without occlusion of the LADC distal to the vessel segment. Despite different applied corrections for artifacts, equation (6.2.3) looks reasonable because of the following advantages:

- the correction factor for the flow leakage is normally close to 1 (a large value of $\lambda=0.3$, renders 1.10 for this correction factor)

- the product of systolic volume flow and the related correction factor is low, since normally systolic coronary artery volume flow is low compared to the diastolic component of flow, and since the related correction factor is close to 1 
- finally, the radius is present to the fourth power, which involves that the relative error in the calculation of the radius $\left(r_{d}\right)$ is one quarter of the relative error in the right part of the equation.

Comparison of the geometry of the coronary trees in the casts and in the in vivo situation

To assess any differences between the geometry of the coronary arteries in yino (casts) and that in vivo, the diameters of corresponding branches as measured in the casts and in vivo were compared. For this comparison a segment of the $L A D C$ distal to the first main side branch was used. The ratio of the diameters in wivo and in wimo was supposed to be a correction factor representative of the whole arterial coronary tree. Additional corrections have to be made for the length of the branches in the coronary trees in relation to the dimensions of the heart. The length of the branches in the arterial coronary trees are assumed to be proportional to the circunference of the heart, which is supposed to be proportional to the cube root of the heart weight, assuming conformity of hearts with different heart weights. The averheart weight in the in vitro experiments $\left(G_{C}\right)$ and the in vivo experiments $\left(G_{v}\right)$ were determined and the correction factor $k_{1}$ for the length is calculated from the following relation:

$$
k_{1}=\sqrt[3]{\frac{G_{v}}{G_{c}}}
$$

The inertance, resistance, volume and capacitance of the epicardial coronary arteries

The resistance, inertance and volume of the epicardial coronary arteries over $0.4 \mathrm{~mm}$ in diameter were computed from the length and diameter of the separate branches. For this purpose admissable simplified versions of the equations, which describe the flow through a cylindrical tube, are used ${ }^{15,24)}$ :

$$
\begin{aligned}
& R_{b}=\frac{8 \pi l_{b}}{\pi r_{b}^{4}} \\
& L_{b}=\frac{4 \rho l_{b}}{3 \pi r_{b}^{2}}
\end{aligned}
$$




$$
v_{b}=\pi 1_{b} r_{b}^{2}
$$

where $R_{b}=$ resistance of a branch

$L_{b}=$ inertance of a branch

$v_{b}=$ volume of a branch

$n=$ viscosity of blood

$\rho=$ density of blood

$l_{b}=$ length of the branch

$r_{b}=$ radius of the branch

The resistance of a tree is defined as the ratio of a pressure difference and the volume flow into the tree. The related pressure difference is the difference between the mean aortic pressure and the mean pressure in the branches of $0.4 \mathrm{~mm}$ in diameter, assuming the same flow in each $0.4 \mathrm{~mm}$ branch. The inertance of a tree is defined as the ratio of the increase in that pressure difference as a result of an increase in volume flow acceleration, and that increase in volume flow acceleration. The resistance, inertance and volume of the whole tree are calculated according to

$$
\begin{aligned}
& \mathrm{R}_{\mathrm{t}}=\frac{1}{\mathrm{~N}_{\mathrm{t}}^{2}} \sum_{\mathrm{k}=1}^{\mathrm{N}_{\mathrm{t}}} \sum_{\text {over all branches in the path from the }}^{\mathrm{N}_{\mathrm{b}}} \\
& \text { entrance to the } k^{\text {th }} \text { branch of } 0.4 \mathrm{~mm} \\
& L_{t}=\frac{1}{N_{t}^{2}} \sum_{k=1}^{N_{t}} \sum_{N}^{N} L_{b} \\
& \text { over all branches in the path from the } \\
& \text { entrance to the } k^{\text {th }} \text { branch of } 0.4 \mathrm{~mm}
\end{aligned}
$$

where $\mathrm{N}_{t}=$ total number of $0.4 \mathrm{~mm}$ diameter branches in the tree

$k=$ serial number of a branch of $0.4\left(1 \leq k \leq N_{t}\right)$

$\mathrm{N}=$ number of distal branches of $0.4 \mathrm{~mm}$ belonging to one branch 
$R_{t}, L_{t}, V_{t}=$ resistance, inertance and volume of the tree.

To evaluate the accuracy of the length and diameter measurements in the casts, the calculated volumes of 16 trees ( 6 LADC, 5 LCC and 5 RC) were compared to the volumes of these trees measured directly by determining the upward force caused by the volume displacement of the tree submerged in water. The capacitance $c_{t}$ of the total tree is calculated from the following equation:

$$
c_{t}=\frac{V_{t}}{\bar{k}}
$$

In deriving this equation, the value of $k$ found by applying equation (6.2.1), is assumed to be representative for all branches of the coronary arterial vasculature. This is indicated by the fact that the ratio of wall thickness to vessel diameter is virtually constant for coronary arteries larger than 0.3 m in diameter ${ }^{3)}$, when assuming that wall material properties are virtually constant in that range of vessel dianeters.

\subsubsection{Results}

The relation between the vessel diameters proximal and distal to a bifurcation

The average number of distal branches of $0.4 \mathrm{~mm}$ in diameter belonging to one branch was determined as a function of the diameter (d) of that branch. Fig. 6.6 shows the empirical data for 12 trees. From 183 data points the following relation was found with a correlation coefficient of 0.989 :

$$
N(d)=\left(\frac{d}{0.38 \mathrm{~mm}}\right)^{2.55}
$$

Equation (6.2.12) renders a relation between the vessel diameters proximal and distal to a bifurcation. When, for instance, branch "prox" bifurcates into branch "dist 1" and "dist 2" (fig. 6.5), the number of distal branches of $0.4 \mathrm{~mm}$, belonging to the former branches are $\mathrm{N}_{\text {prox }}$. $\mathrm{N}_{\text {dist } 1}$ and $\mathrm{N}_{\mathrm{dist}}$, respectively. Obviously it holds that

$$
N_{\text {prox }}=N_{\text {dist } 1}+N_{\text {dist } 2}
$$

Substitution of the experimental relation (6.2.12) into relation (6.2.13) 


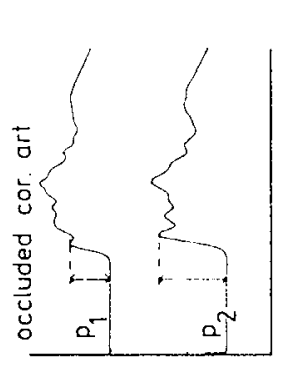

БHWW 0 S

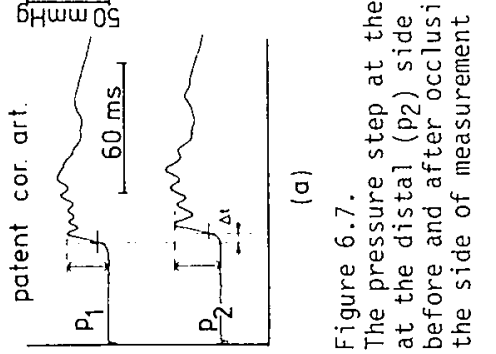

突芯号

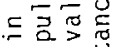

б幽芯

总寻市次

중 \&4

立吉管

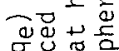

의

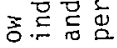

的的

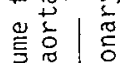
$>$ 웡

등 $=\frac{1}{5}$

ज्ञ

$\infty$ 워웅

6 \&

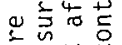

ㄴํㅇㅇㅇ

首题

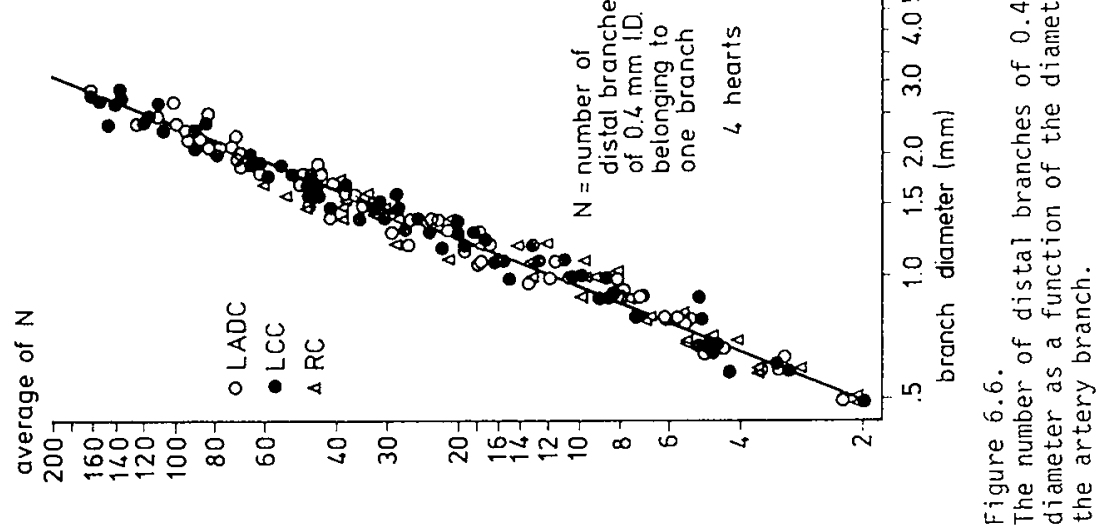




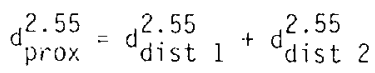

For a symetric bifurcation this relation implies a ratio between the proximal and distal branch diameters of 1.31 which agrees perfectly with the theoretical value for minimum reflection at the bifurcation, also being 1.31 (appendix $6.1)$. An indication of the sensitivity of the reflection coefficient to variations in this ratio is shown by fig. A 6.1. This figure shows larger reflections at low frequencies, if the parameter $s=2.5$, which involves a value of the ratio mentioned of 1.32 .

\section{Reflections}

In fig. 6.2 the recordings of an in the aorta induced pressure pulse in a patent and a distally occluded $L A D C$ are shown. In a patent coronary artery (fig. 6.7 a) the wave form of the proximal and distal pressure pulses are similar, which indicates that under these circunstances hardly any reflections are present. However, after occlusion of the LAOC distal to the pressure transducers, reflections are observed as demonstrated by the increase in amplitude of the ascending part of the distal pressure pulse. In fig. 6.7 b the amplitude of the distal pressure pulse has approximately twice the value of the proximal one, which is mainly due to total reflection at the site of occlusion.

However, it should be noticed that the applied method to study reflections is not sensitive to low frequencies. For this purpose reflections are studied at different values of the coronary peripheral resistance. In the aorta a pressure pulse is induced and pressure and volume flow at the entrance of the LADC are measured.

From the theory on wave guiding and reflection in transmission lines ${ }^{17}$ ) it follows that a wave is not reflected if the hemodynamic impedance at the entrance is equal to the characteristic impedance of the vessel at the entrance, i.e. the LADC. This characteristic impedance is determined by the physical properties of the $\angle A D C$ and, therefore, is independent of the peripheral load of the LADC. If in the experimental situation the input impedance of the LADC appears to depend on the coronary peripheral resistance, it is demonstrated that the reflection coefficient depends on the peripheral resistance and, hence, reflections exist.

The unbroken trancings in fig. 6.8 are the recordings of pressure and volume flow in a proximal part of the LADC when a pressure pulse is induced in 
the aorta. The dotted tracings in fig. 6.8 represent these responses in the situation that coronary peripheral resistance is approximately half the normal value. This intervention is effected by intravenous injection of dipyridamole, a coronary vasodilator. Despite the similarity of the pressure recordings, the recordings of coronary artery flow appears to be different, which implies that the input impedance of the $\angle A D C$ has changed and, hence, reflections exist. The rising slope of the volume flow is approximately the same in both situations, which indicates that reflections at high frequencies are not affected by changes in peripheral resistance. Analys is of the difference between both flow recordings reveals that components in the signal below $7 \mathrm{~Hz}$ are significantly reflected by the coronary peripheral resistance. Reflection of these low frequencies may be due to the related long wave length as conpared with the dimensions of the coronary system. From this analys is it may be conciuded, that at high frequencies $(>7 \mathrm{~Hz}$ ), reflections in the coronary system are low and independent of coronary vasodilation, induced by dipyridamole.

\section{Determination of the cross-sectional stiffness}

The cross-sectional stiffness of the vessel seginent of the LAOC is computed from the wave front velocity, using equation (6.2.1). Since the wave front velocity is a function of arterial blood pressure, this velocity was determined as a function of the 25 pressure level of the step. In each dog the data were grouped according to the various pressure levels so that for some dogs more than one group was obtained. The mean wave front velocities and the corresponding $95 \%$ reliability interval were computed. The results are shown in fig. 6.9. At the lower pressure levels the reliability interval is smaller and the density of measurements higher. Thence, the best fitting logarithmic curve through all groups of data was computed just as the correlation coefficient. The following relation was found:

$$
v_{w f}=0.0123 p^{0.69} \text { (correlation coefficient } 0.86 \text { ) }
$$

where $v=$ wave front velocity in $\mathrm{m} \mathrm{s}^{-1}$ and

$p=$ coronary pressure in $\mathrm{N} \mathrm{m}^{-2}$

For a pressure of $1.33 \cdot 10^{4} \mathrm{~N} \mathrm{~m}^{-2}(100 \mathrm{mmHg})$ it follows

$$
v_{w f}=8.6 \pm 1.4 \mathrm{~m} \mathrm{~s}^{-1} \text { (mean } \pm \mathrm{s} \text { ) }
$$

where $s$ = standard deviation. 


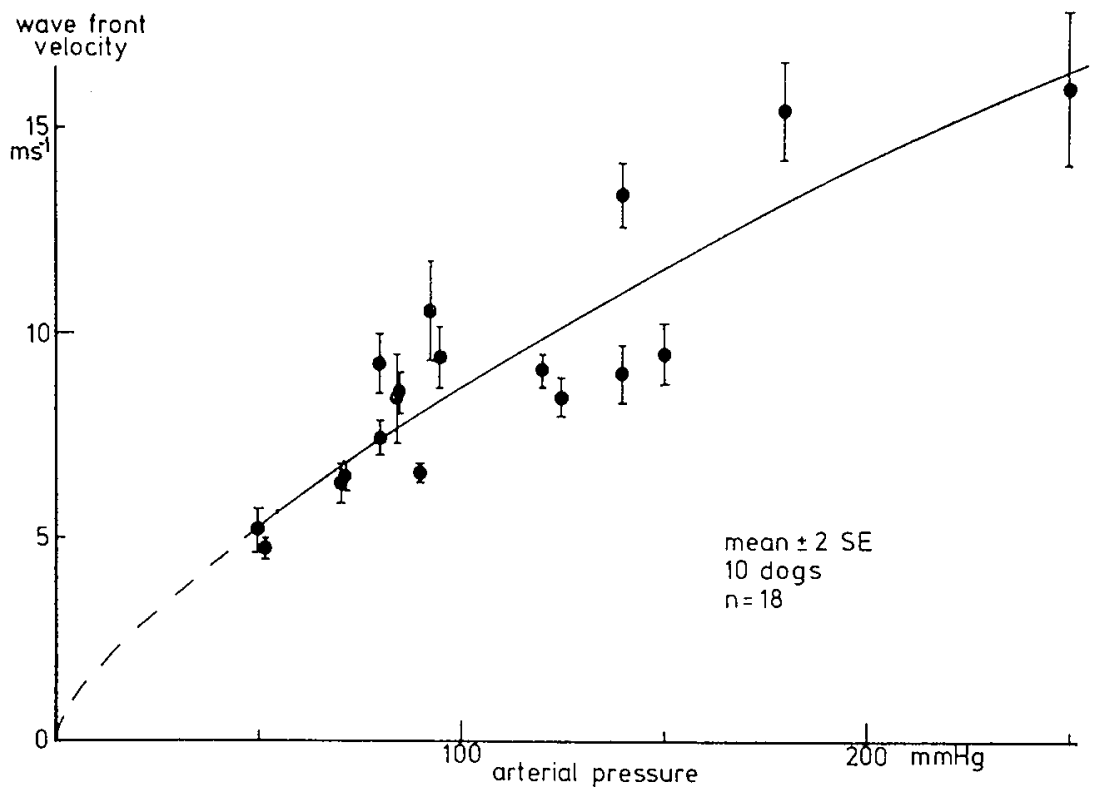

Figure 6.9.

Wave front velocity as a function of coronary artery blood pressure, determined at 25 of the maximum of the pressure step.

Figure 6.10.

Comparison of the volume of the coronary casts as calculated with those obtained by measurement.

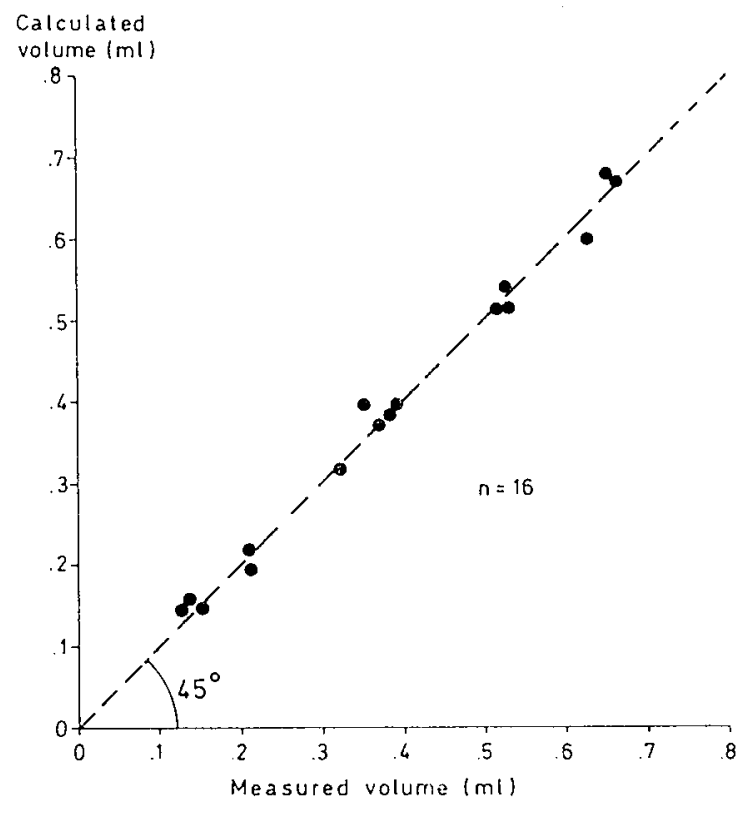


The cross-sectional stiffness is computed as

$$
K=9.7 \cdot 10^{4} \pm 3.3 \cdot 10^{4} \mathrm{~N} \mathrm{~m}^{-2}(\text { mean } \pm \mathrm{s})
$$

at a coronary artery pressure of $1.33 \cdot 10^{4} \mathrm{~N} \mathrm{~m}^{-2}$ (100 $\mathrm{mmHg}$ ). A pressure step from $1.33 \cdot 10^{4} \mathrm{~N} \mathrm{~m}^{-2}(100 \mathrm{mmHg})$ up to $2.01 \cdot 10^{4} \mathrm{~N} \mathrm{~m}^{-2}(150 \mathrm{mmHg})$ resulted in an increase of the cross-sectional area of $5.1 \div \pm 1.7 \%$ (mean \pm 5 ). From now on this value of $k$ is used for all coronary arteries modelled.

Comparison of the dimensions in vitro (casts) and in vivo

A fair agreement was found between the diameters of the LADC distal to the first large side branch in the casts and in vivo as normalized for an arterial pressure of $100 \mathrm{mmHg}$. These diameters are $1.00 \pm 0.11 \mathrm{~mm}$ (mean $\pm \mathrm{s}$ ) and 1.00 $\pm 0.13 \mathrm{~mm}$ (mean $\pm \mathrm{s}$ ), respectively. From this result it is concluded that no diameter corrections are necessary for the determination of the impedance $i n$ Wo. However, corrections of the length of the vessels still nust be made. The casts were obtained from hearts with a mean weight $G_{C}$ of $123 \mathrm{~g}$. The mean heart weight of the dogs in the in vino experiments $\left(G_{v}\right)$ was $230 \mathrm{~g}$. According to relation (6.2.4) the lengths measured in the casts have to be multiplied by $\sqrt[3]{G_{V} / G_{C}}=1.23$. The mean values and the standard deviations of the resistance, inertance and volume of the three coronary trees, based on the dimensions of the cast without corrections are presented in table 6.1. The length and diameter measurements in the casts are accurate as indicated by the excellent agreement between the volumes computed from these dimensions and the volumes measured in a direct way (fig. 6.10). The mean values and standard deviations of the resistance, inertance, volume and capacitance of the three coronary trees, corrected for differences in dimensions between the casts and the in vivo situation are presented in table 6.2.

In the present model of the epicardial coronary arteries the inertance is assumed to be localized in the proximal part of the epicardial coronary arteries (fig. 3.3), since the larger proximal vessels dominate this inertance. The assumption is made that the resistance is equally distributed over the coronary arterial vasculature, whereas the capacitance is localized halfway these vessels. At frequencies below $15 \mathrm{~Hz}$ this network is a good representative of the real situation.

\subsubsection{Discussion}

The values for the inertance, resistance, volume and capacitance of the epi- 
Table 6.1:

The mean values and the standard deviations of the resistance, inertance and volume of the epicardial coronary arteries obtained from 9 araldite casts

BRANCH

\begin{tabular}{ll} 
& $\frac{\left(10^{8} N s^{2} m^{-5}\right)}{\bar{x}}$ \\
\hline LAOC & $\frac{0.112 \pm 0.025}{0.02} \pm 0.053$ \\
LCC & $0.159 \pm 0.055$ \\
RC & $0.244 \pm 0$
\end{tabular}

RESISTANCE

\begin{tabular}{|c|c|c|}
\hline $\bar{x}$ & & $s$ \\
\hline 8.7 & \pm & 2.7 \\
\hline 9.6 & \pm & 3.2 \\
\hline 25.6 & + & 8.4 \\
\hline
\end{tabular}

VOLUME

(ml)

$\overline{\bar{x}}+\frac{s}{0.49 \pm 0.14}$
$0.63 \pm 0.17$
$0.19 \pm 0.08$

HEART WEIGHT $123 \pm 34 \mathrm{~g}$ (mean $\pm \mathrm{s})$

Table 6.2:

The mean values and standard deviations of the inertance, resistance, capacitance and volume of the epicardial coronary arteries after correction for in vitro - ir. vivo differences, obtained from a group of 10 dogs. The data are given at an arterial pressure of $1.33 \cdot 10^{4} \mathrm{~N} \mathrm{~m}^{-2}(100 \mathrm{mmHg})$.

\begin{tabular}{|c|c|c|c|c|c|c|c|c|c|c|c|}
\hline \multirow[t]{3}{*}{ BRANCH } & \multirow{2}{*}{\multicolumn{2}{|c|}{$\begin{array}{c}\text { INERTANCE } \\
\left(10^{8} \mathrm{~N} \mathrm{~s}^{2} \mathrm{~m}^{-5}\right)\end{array}$}} & \multicolumn{3}{|c|}{ RESISTANCE } & \multicolumn{3}{|c|}{ CAPACITANCE } & \multicolumn{3}{|c|}{ VOLUME } \\
\hline & & & $\left(10^{8}\right.$ & $\mathrm{NS}$ & $\left.m^{-5}\right)$ & $\left(10^{\circ}\right.$ & N & $\left.m^{5}\right)$ & & mi) & \\
\hline & $\bar{x}$ & $s$ & $\bar{x}$ & & $\mathrm{~s}$ & $\bar{x}$ & & $s$ & $\bar{x}$ & & $s$ \\
\hline LADC & 0.137 & \pm 0.047 & 10.7 & \pm & 3.9 & 6.2 & \pm & 2.8 & 0.60 & \pm & 0.19 \\
\hline LCC & 0.195 & \pm 0.081 & 11.8 & \pm & 4.6 & 7.9 & \pm & 3.5 & 0.77 & \pm & 0.23 \\
\hline $\mathrm{RC}$ & 0.300 & \pm 0.103 & 31.5 & \pm & 12.1 & 2.4 & \pm & 1.3 & 0.23 & \pm & 0.10 \\
\hline
\end{tabular}

HEART WEIGHT $230 \pm 51 \mathrm{~g}$ (mean $\pm \mathrm{s}$ ) 
cardial coronary arteries of the dogs as determined in the present study are considered to be representative for the in vivo situation. Apart from the capacitance these values are calculated from the diameter and length of the relevant vessels as obtained from araldite casts. Correction factors are applied for the differences in geometry of the coronary arteries in vitro and in vivo.

Although the casts were made from small hearts with a weight of $123 \mathrm{~g} \pm 34 \mathrm{~g}$ (mean $\pm s$ ), the diameter of the $\angle A D C$ distal to the first side branch was not significantly different from the value determined in vivo in dogs with heart weights of $230 \mathrm{~g} \pm 51 \mathrm{~g}$ (mean $\pm \mathrm{s}$ ). This justifies that no corrections are made with respect to the diameter. The relatively large diameters in the casts probably result from the process of casting in combination with postmortem changes. It seems acceptable to assume that the ratio of the diameters as determined in vivo and in vitro in a segment of the LADC is representative of the ratio of the diameters of corresponding branches in the whole coronary tree, since the ratio of wall thickness to radius is constant and the pressure during casting prior to hardening is virtually independent of the site in the arterial tree. The latter statement is indicated by the fact that the flow of araldite prior to hardening is viscous. This implies maximum flow resistance in the far distal parts (arterioles) of the arterial vasculature, whereas the pressure drop in the proximal larger vessels is negligible.

After correction for the differences in vivo and in vitro the volume of the left coronary artery and its branches was found to be $0.60 \pm 0.04 \mathrm{ml}$ (mean \pm $\mathrm{SE}$ ) per $100 \mathrm{~g}$ heart tissue. This volume is significantly less than reported by Douglas et a 1.6 ). These investigators found a volume of $1.30 \pm 10 \mathrm{ml}$ (mean $\pm \mathrm{SE}$ ) per $100 \mathrm{~g}$ heart tissue for the left coronary artery and its branches. This discrepancy can partly (approximately 20\%) be explained by the fact that in their study the smaller arteries (down to $200 \mu \mathrm{m}$ in diameter) are included, whereas in the present work the range is restricted down to $400 \mu \mathrm{m}$ in diameter. It should also be stated that Douglas and co-investigators did not make corrections for the differences in vivo and in vitro.

No systematical data on the resistance of the epicardial coronary arteries could be found in literature. The value of the resistance in the LADC as determined in the present study $\left(10.7 \cdot 10^{8} \pm 3.4 \cdot 10^{8} \mathrm{Nsm}^{-5}\right.$; mean $\left.\pm \mathrm{s}\right)$, however, is in agreement with the resistance values (ranging between $6 \cdot 10^{8}$ to $12 \cdot 10^{8}$ $\mathrm{Nsm}^{-5}$ ) calculated from the flow and pressure measurements as described by Reneman et al. 19).

No data could be found in literature on the inertance of the epicardial cor- 
onary arteries. The data resulting from the present work are likely to be correct. The inertance, afterall, is mainly determined by the diameter and length of the separate branches of the coronary vascular tree. These quantities were determined reliably as appears from the close correlation between the calculated and directly measured volumes of the casts.

In the present study the dynamic capacitance of a coronary artery and its branches is computed from the volume and the cross-sectional stiffness. As to this the wave front velocity is calculated in a part of the LADC with an initial internal dianeter of $2 \mathrm{~mm}$ and tapering down to approximately $1.6 \mathrm{~mm}$. This constant value for the cross-sectional stiffness is used for the calculation of the dynamic capacitance of all coronary arteries with an internal diameter ranging between 0.4 and $2.5 \mathrm{~mm}$, neglecting the fact that wall properties change with vessel diameter 20$)$. Probably the error made by this assumption is small since for coronary arteries with an internal diameter between 0.3 and 4 $\mathrm{mm} 3$ ) the ratio of vessel wall thickness to vessel diameter is independent of the vessel diameter, and wall material properties are presumed to be constant. Moreover, the volume of the coronary arteries with a diameter over $1 \mathrm{~mm}$ appears to amount to $65 \%$ of the total volume. Hence the capacitance is mainly determined by the large branches, of which the segment of the LADC is likely to be a sample. A large value of the capacitance corresponds with a low value of the cross-sectional stiffness.

The dynamic cross-sectional stiffness presently found $\left(K=9.7 \cdot 10^{4}\right.$ $\pm 1.1 \cdot 10^{4} \mathrm{~N} \mathrm{~m}^{-2}$; mean $\pm \mathrm{SE}$ ) for an arterial pressure $\mathrm{p}$ of $1.33 \cdot 10^{4} \mathrm{~N} \mathrm{~m}^{-2}(100$ $\mathrm{mmHg}$ ) is larger than the data on the static cross-sectional stiffness, derived from the studies of Patel et al. ${ }^{16)}\left(K=3.0 \cdot 10^{4} \mathrm{~N} \mathrm{~m}^{-2}, p=1.67 \cdot 10^{4} \mathrm{~N} \mathrm{~m}^{-2}\right)$ and Douglas et a1. $\left.{ }^{6}\right)\left(K=1.5 \cdot 10^{4} \mathrm{~N} \mathrm{~m}^{-2}, p=1.26 \cdot 10^{4} \mathrm{~N} \mathrm{~m}^{-2}\right)$. However, Gow et a). ${ }^{8)}$ found a larger value for the static cross-sectional stiffness ( $K=$ $12.2 \cdot 10^{4} \mathrm{~N} \mathrm{~m}^{-2}, p=1.33 \cdot 10^{4} \mathrm{~N} \mathrm{~m}^{-2}$ ).

A value for the dynamic capacitance less than the static capacitance is in accordance with the findings of Douglas et al. ${ }^{6}$ ) and Gow et al. 8 ). This difference between static and dynamic capacitance also exists in systemic arteries $^{4,8)}$.

The dynamic capacitance of the coronary arteries is larger than the one of the systemic arteries of the same size ${ }^{6}$. Thence, the wave front velocity in the coronary arteries will be less than in equally sized systemic arteries. The average value for the wave front velocity in the coronary arteries found in the present study $\left(8.6 \mathrm{~m} \mathrm{~s}^{-1}\right)$ is less than reported for the femoral artery 
(12 $\mathrm{m} \mathrm{s}^{-1}$ ) at the same arterial pressure level of $1.33 \cdot 10^{4} \mathrm{~N} \mathrm{~m}^{-2}$ (100 mmg $)^{14)}$. Douglas et al. ${ }^{6}$ ) as well as Gow et al. ${ }^{8)}$ concluded to a stiffer dynamic behavior of the coronary arteries $\left(K=17.6 \cdot 10^{4}+2.9 \cdot 10^{4} \mathrm{~N} \mathrm{~m}^{-2}\right.$; mean \pm SE, and

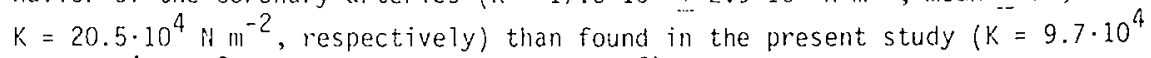
$\pm 1.1 \cdot 10^{4} \mathrm{~N} \mathrm{~m}^{-2}$; mean $\pm \mathrm{SE}$ ). Douglas et al. ${ }^{6}$ ) occluded the peripheral coronary arteries by 200 im glass beads and detemined the relation between pressure and volume of the proximal part of the coronary vasculature. The differences with the present results can partly be explained by a lower elasticity of the smaller vessels down to $0.2 \mathrm{~mm}$ in diameter included in their study, because this results in a lower capacitance of the arterial tree under investigation. Furthermore their calculations are subject to errors. Firstiy they substituted too large pressure and time differences into the differential equation of their capacitance-resistance model, which brings about an underestination of the capacitance. On the basis of their model this underestimation can readily amount to $50 \%$. With their method of computation and using the data presented in figure 2.4 of their study ${ }^{6}$ ), even negative capacitance values are found. Secondly they probably overestimated the volume of the coronary arteries, since they did not correct for differences in vitro and in vivo in the diameter of these vessels. An overestimation of the volume results in an underestimation of the relative volume increase.

The values for the static as well as for the dynamic capacitance found by Gow et al. ${ }^{8)}$ are low. They determined the relation between pressure and diameter of large coronary arteries. In their study the coronary arteries are not surrounded by connective tissue while the mechanical properties of the arteries might be changed because of the long period (at least $45 \mathrm{~min}$ ) of waiting until a steady state was reached. Both these factors will change the dynamic capacitance of the arteries.

The analysis in the present study gives an explanation for the absence of reflection of high frequency components $(>7 \mathrm{~Hz})$ during diastole in patent coronary arteries. The ratio between the vessel diameters proximal and distal to a symmetric bifurcation was found to be 1.31 which agrees very well with the theoretical value for minimum reflections in a bifurcation. Reflection of high frequency components $(>7 \mathrm{~Hz})$ can be introduced by occluding the LADC distally, but not by dilating the coronary vasculature. At low frequencies $(<7 \mathrm{~Hz})$ reflections at the coronary peripheral resistance are more pronounced. 


\subsection{The coronary microcirculation}

Knowledge of the hemodynamic behavior of the coronary microcirculation is 1 imited. In the present model the presumed characteristics of the coronary microcirculation are based mainly on basic information obtained from textbooks. In each subcylinder the coronary microcirculation is simulated by an arteriolar, capillary and venular subcompartment. This subdivision in compartments is based on differences in function and physical properties.

The arteriolar subcompartment controls the volume flow into the capillaries by vasoactivity. The vasoactivity and, therefore, the flow resistance, is regulated by contraction of smooth muscle cells in the arteriolar walls, which causes a decrease in diameter of the arterioles. Normally, therefore, the pressure drop in the arterioles is large.

In the capillary subcompartment exchange of substrates with the tissue takes place. Capillaries are narrow $(: 4.4 \mathrm{\mu m}) 10)$, long $\left.(\approx 0.5 \mathrm{~mm})^{2}\right)$ and densely distributed $\left(\simeq 3200-3800\right.$ capillaries $\left.\left.\mathrm{mm}^{-2}\right) 2\right)$. Contractile proteines have been found in low concentrations in the capillary endothelium ${ }^{25)}$, but in the present model vasoactivity of capillaries is neglected. The transmural pressure is rather low and a normal value of the pressure drop in the capillaries in only $10 \mathrm{minh}^{21)}$.

The venular subcompartment collects the blood from the capillaries. In the present model the blood in the venules is assumed to reach the right atrium through the large coronary veins. Data in literature ${ }^{23)}$, however, reveal that part of the venular blood flows directly into the atria, the right ventricle and even into the left ventricle. The distal pressure of the venular subcompartment is assumed to be equal to the maximum value of either the right atrial pressure or the intramyocardial pressure. This assumption considers the effect of the collapsibility of the venules and veins ${ }^{7}$.

In the present model a subcompartment in the microcirculation is simulated as shown in fig. 6.11. The volume $V_{c}$ of the subcompartment, which is a characteristic quantity (chapter 4 ), determines the transmural pressure $p_{t r}$

$$
p_{t r}\left(v_{c}\right)=p_{c}-p_{i m}
$$

where $p_{c}=$ average pressure in the subcompartment

$p_{i m}=$ intramyocardial pressure

The function $p_{t r}\left(v_{c}\right)$ is introduced as 


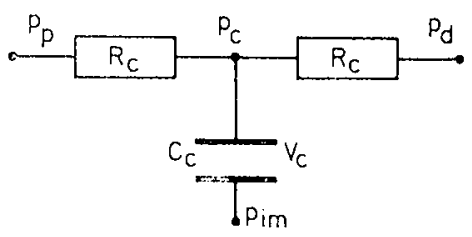

Figure 6.11.

Network which represents a coronary microcirculatory subcompartment. $p p=$ proximal pressure; $p_{d}=$ distal pressure; $p_{i m}=$ intramyocardial pressure; $R_{c}=r e-$ sistance; $C_{C}=$ capacitance; $V_{C}=$ volume.

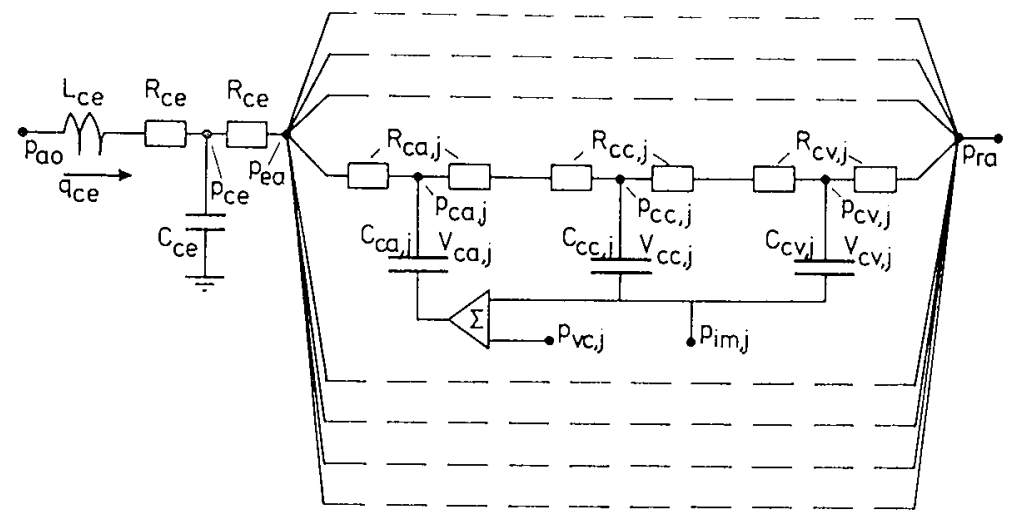

Figure 6.12.

Network which represents the coronary circulation. $\mathrm{R}=$ resistance; $C=$ capacitance; $L=$ inertance; $V=$ volume; $p=$ pressure.

Subscripts: ce - large coronary arteries (I.D. > 400 um)

ca - coronary arteriolar subcompartment

cc - coronary capillary subcompartment

cv - coronary venular subcompartment

ra - right atrium + large veins

im - intramyocardial

vc - vasoconstrictive

and $j=$ ordinal number of the subcylinder. 


$$
p_{t r}\left(v_{c}\right)=S_{p} V_{c}{ }^{S}{ }_{C} \text { with } s_{c} \gg 1
$$

where $s_{c}=$ parameter governing the elasticity of the vessel

$S_{p}=$ proportionality factor.

The characteristics of this relation are:

- low volume corresponds to $p_{t r} \approx 0$

- when the volume $V_{c}$ increases, the stiffness of the vessel increases rapidly. The pressure $\left(\mathrm{p}_{\mathrm{c}}\right)$ in the subcompartment is computed from the transmural pressure and the intramyocardial pressure, which is calculated according to the relations in chapter 7 . The volume of the compartment determines the resistance $\left(R_{c}\right)$ in the subcompartment according to Poisseuille's law, if it is assumed that the length of the vessels is constant. This results in

$$
R_{c}=\frac{S_{r}}{V_{c}^{2}} \quad \text { (Poisseuille) }
$$

Where $S_{r}$ is a proportionality factor of the resistance $R_{c}$ in relation to the volume of the compartment. The volume inflow $q_{p}$ and outflow $q_{d}$ are calculated according to

$$
\begin{aligned}
& q_{p}=\frac{\max \left(p_{p}, p_{i m}\right)-\max \left(p_{c}, p_{i m}\right)}{R_{c}} \\
& q_{d}=\frac{\max \left(p_{c}, p_{i m}\right)-\max \left(p_{d}, p_{i m}\right)}{R_{c}}
\end{aligned}
$$

where $p_{p}$ and $p_{d}$ are the pressure at the proximal and distal side of the compartment, respectively. As said before, the maximum values consider the effect of the collapsible behavior of bloodvessels. For the derivative of the characteristic quantity $v_{c}$ it holds

$$
\frac{d v_{c}}{d t}=q_{p}-q_{d}
$$

Some modifications of the relations which describe the behavior of a specific subcompartment are necessary:

- the arterialar subcompartment regulates the volume flow by vasoconstriction, 
which is simulated by a vasoconstrictive pressure superimposed to the intramyocardial pressure (chapter 3 );

- the capillaries run parallel to the muscle fibers. Because of this, the length of the capillaries is assumed to be proportional to the sarcomere length, which affects the resistance;

- during a simulated heart cycle only the distal end of the venular subcompartment collapses thus considering the effect of collapsing of venules as well as veins. For this reason only the distal pressure of the venular compartment is compared with the intramyocardial pressure, and the highest of both pressures is considered to be the effective pressure at the distal side of the subcompartment.

\subsection{Mathematical representation of the coronary circulation}

Fig. 6.12 shows in diagram the coronary circulation as represented by the model. The present section deals with the sequence of calculations of the derivatives of the relevant characteristic quantities.

The derivative of the flow into the large coronary arteries $\left(q_{c e}\right)$, which is a characteristic quantity, is

$$
\frac{d q_{c e}}{d t}=\frac{1}{L_{c e}}\left(p_{a o}-p_{c e}-\frac{1}{2} q_{c e}{ }^{R} c e\right)
$$

where $P_{a 0}=$ aortic pressure

$p_{c e}=$ epicardial coronary pressure

$L_{c e}=$ inertance of the epicardial coronary arteries

$R_{c e}=$ resistance of the epicardial coronary arteries.

The computation of $p_{a o}$ is discussed in chapter 7 . The pressure $p_{c e}$ is given by

$$
p_{c e}=\frac{1}{c_{c e}}\left(v_{c e}-v_{c e o}\right)
$$

where $V_{c e}=$ volume of the epicardial coronary arteries

$C_{c e}=$ capacitance of the epicardial coronary arteries

$v_{\text {ceo }}=$ extrapolated volume $v_{c e}$ for $p_{c e}=0$.

After leaving the epicardial coronary arteries the flow is distributed over the subcylinders. For the volume flow $q_{e a, j}$ into the arteriolar compartment of subcylinder $j$ it holds 


$$
q_{e a, j}=\frac{p_{e a}-p_{c a, j}}{R_{c a, j}}
$$

where $\mathrm{p}_{\mathrm{ea}}=$ pressure distal to the arterial coronary compartment

$\begin{aligned} R_{C a, j}= & \text { half the resistance of the arteriolar subcompartment in subcy }- \\ & \text { inder } j\end{aligned}$

$p_{c a, j}=$ pressure half way the arteriolar subcompartment in subcylinder $j$. For the pressure $p_{\text {ea }}$ it holds

$$
p_{e a}=\frac{\sum_{j=1}^{n} \hat{R}_{c a, j}+\frac{2 p_{c e}}{R_{c e}}}{\sum_{j=1}^{n} \frac{1}{R_{c a, j}}+\frac{2}{R_{c e}}}
$$

where $n=$ number of subcylinders.

According to relation (6.3.3) $\mathrm{R}_{\mathrm{Ca}, \mathrm{j}}$ is calculated from the volume of the corresponding arteriolar compartment, which is the characteristic quantity $V_{c a, j}$. For the pressure $p_{c a, j}$ it holds

$$
p_{c a, j}=p_{i m, j}+p_{v c, j}+p_{t r a, j}
$$

where $p_{i m, j}=$ intramyocardial pressure in subcylinder $j$

$p_{v c, j}=$ vasoconstrictive pressure in subcylinder $j$

$p_{\text {tra }, j}=$ average value of the pressure over the arteriolar walls in subcylinder $j$.

The transmural pressure $p_{\text {tra, } j}$ is calculated according to relation (6.3.2) from the volume $v_{c a, j}$. The derivative of the volume of the coronary arteries is

$$
\frac{d V_{c e}}{d t}=q_{c e}-\sum_{j=1}^{n} q_{e a, j}
$$

The flow from the arteriolar compartment into the capillary compartment is

$$
q_{a c, j}=\frac{p_{c a, j}-p_{c c, j}}{R_{c a, j}+R_{c c, j}}
$$

where $R_{c c, j}=$ half the resistance of the capillary subcompartment in subcyl- 
inder $j$

$P_{c c, j}=$ pressure half way the capillary subcompartment in subcylinder $j$. For the flow into the venular compartment it holds

$$
q_{c v, j}=\frac{p_{c c, j}-p_{c V, j}}{R_{c c, j}+R_{c V, j}}
$$

where $R_{c v, j}=$ half the resistance of the venular subcompartment in subcylin$\operatorname{der} j$

$p_{C V, j}=$ pressure half way the venular subcompartment in subcylinder $j$. The outflow of the venular subcompartment is

$$
q_{v r, j}=\frac{p_{c v, j}-\max \left(p_{i m, j}, p_{r a}\right)}{R_{c v, j}}
$$

where $p_{\text {ra }}=$ pressure in the right atrium.

The pressures $p_{C C, j}$ and $p_{C V, j}$ are found to be

$$
\begin{aligned}
& p_{c c, j}=p_{i m, j}+p_{\text {trc }, j} \\
& p_{c v, j}=p_{i m, j}+p_{t r v, j}
\end{aligned}
$$

where both the capiliary and venular transmural pressures $p_{t r c, j}$ and $p_{\text {trv }, j}$ are calculated from the volumes $V_{c c, j}$ and $V_{c V, j}$, respectively, according to relation (6.3.2). In an analogous way the resistances $R_{c c, j}$ and $R_{c v, j}$ are calculated from $V_{c c, j}$ and $V_{c v, j}$ according to relation (6.3.3). The derivatives of $V_{c c, j}$ and $V_{c v, j}$ are

$$
\begin{aligned}
& \frac{d v_{c c, j}}{d t}=q_{a c, j}-q_{c v, j} \\
& \frac{d v_{c v, j}}{d t}=q_{c v, j}-q_{v r, j}
\end{aligned}
$$

Yet in the simulation all derivatives of the characteristic quantities, which are relevant for the coronary circulation, are calculated. 


\section{Literature}

1. Arts, T., Reneman, R.S.: Resistance, inertance, voluma and capacitance of the epicardial coronary arteries in dogs. Fed Proc 33, 332 (1974).

2. Bassingthwaighte, J.B., Yipintsoi, T., Harvey, R. B.: :Hcrovasculature of the dog left ventricular myocardium. Microvasc Res 7. 229 - 249 (1974).

3. De Brabander, M., Schaper, H.: Quantitative histology of the canine coronary collatoral circulation in localized myocardial ischemia. Life Sci 10, $857-868(1971)$.

4. Bergel, D.H.: The dynamic elastic properties of the arterial wall. J Physiol (London) 156, $458-469$ (1961).

5. Van Citters, R.L., Wagner, B.H., Rushmer, R.F.: Architecture of small arteries during vasoconstriction. Circ Res 10, $668-675$ (1962).

6. Douglas, J.E., Greenfield, J.C.: Epicardial coronary artery compliance in the dog. Circ Res 27, 921 - 929 (1970).

7. Downey, J.M., Downey, H.F., Kirk, E.S.: Effects of myocardial strains on coronary bloodflow. Circ Res 34, 286 - 292 (1974).

8. Goedhart, W.J.A.: Visco-elastische eigenschappen van de arterie wand. Thesis, University of Amsterdam (1971).

9. Gow, B.S., Schonfeld, D., Patel, D.J.: The dynamic elastic properties of the canine left circuinflex artery. J Biomech 7, $389-395$ (1974).

10. Henquel1, L., Lacelle, P.L., Honig, C.R.: Capillary diameter in rat heart in situ: Relation to erythrocyte deformability, of transport, and transmural 02 gradients. Hicrovasc Res 12, $259-274$ (1976).

11. Jahnke, Ende, Lösch: Tables of higher functions. Stuttgart, Teubner (1966).

12. Lewi, P.I., Schaper, W.K.A.: The estimation of coronary volume elasticity in the beating heart of the dog. Pflueger Arch Ges Physiol 325, 191 - 198 (1971).

13. Harsboom, R.A., Verstraete, D., Thienpont, liattheeuws, D.: The use of haloanisone and phentanyl for neuroleptanalgesia in dogs. Br Vet $\mathrm{J} 120$, $466-468(1964)$.

14. McDonald, D.A.: Regional pulse-wave velocity in the arterial tree. J Appl Physiol 24, $73-78$ (1968).

15. McDonald, D.A.: Bloodflow in arteries. 2nd ed. The Camelot Press Ltd, Southampton (1974).

16. Patel, D.J., Janicke, J.: Static elastic properties of the left circumflex artery and the common coratid artery in dogs. Circ Res 27, $149-158$ (1970).

17. Pender- Hc. I lwain,: Electrical Engineers Handbook. New York: Hiley, New York, 4th ed. 5, $22-26$ (1954). 
18. Reneman, R.S., Clarke, H.F., Simions, N., Spencer, H.P.: In vivo comparison of electromagnetic and doppler flowmeters with special attention to the processing of an analogue doppler flow signal. Cardiovasc Res 7, $557-566$ (1973).

19. Reneman, R.S., Spencer, M.P.: The use of diastolic reactive hyperemia to evaluate the coronary vascular system. Ann Thorac Surg $13,477-487$ (1972).

20. Rushmer, R.F.: Cardiovascular dynamics. Second edition, 102. 1!. B. Saunders Comp. Philadelphia and London (1961).

21. Smaje, L., Zweifach, B.W., Integliatta, M.: Micropressure and capillary filtration coefficients in single vessels of the cremaster muscle of the rat. Microvasc Res 2, 96 - $110(1970)$.

22. Spencer, M.P., Edmunds, L.H.: Evaluation of operative left ventricular outflow tract lesions with a fluid impedance plot. Circulation 37, 912 921 (1968).

23. Tamura, K., Laks, M.M., Garner, D., Swan, H.J.C.: Distribution rates of coronary inflow and outflow in the post-mortem canine heart. Cardiovasc $\operatorname{Res} 3,324$ - 330 (1969).

24. Womersley, J.R.: Method for the calculation of the velocity, rate of flow and viscous drag in arteries when the pressure gradient is known. J Physiol (London) 127, 553 - 563 (1955).

25. Yohro, T., Burnstock, G.: Filament bundles and contractility of endothelial cells in coronary arteries.Z Zellforsch 138, 85 - 95 (1973). 
7. OETERMINATION OF THE IMSTANTANEOUS STRESSES AND STRAINS IN THE WALL OF THE CYLINDER

\subsection{Introduction}

As mentioned in chapter 4 the stresses and strains in the wall of the cylinder, which represents the left ventricle, are determined by the volume of the cavity enclosed by the cylinder, the instantaneous values of the quantities, which describe the local contractile state (chapter 5) of the muscle material, and the local volume of the coronary vasculature. The present chapter deals with the calculation of the left ventricular pressure, dimensions and torsion angle of the cylinder, local sarcomere length and local intramyocardial pressure. The torsion angle is is defined as the angle over which the upper cross-sectional surface of the cylinder is rotated around the axis of the cylinder with respect to the lower cross-sectional surface of the cylinder. In fig. 7.1 a blockdiagram of the calculation sequence is shown.

In section 7.2 the dimensions and torsion angle of each subcylinder are calculated from the left ventricular cavity volume and the coronary volume in each subcylinder after estimating the torsion angle and height of the cylinder. In section 7.3 the sarcomere length, fiber angle and stresses in each subcylinder are calculated. In section 7.4 the equilibrium of forces and torques acting on the upper surface of the cylinder are considered. New estimates of the torsion angle and the height are derived from the imbalances. In section 7.5 left ventricular cavity pressure and local intramyocardial pressures are calculated.

\subsection{Determination of dimensions and torsion angle of each subcylinder}

In chapter 3 it is mentioned that the thick-walled cylinder is composed of thin-walled concentric subcylinders. The subcylinders are numbered off from the inner subcylinder. Independent of the state of deformation the following holds:

- the subcylinders remain in contact with each other, which is expressed by

$$
r_{0, j}=r_{i, j+1} \quad 1 \leq j \leq n-1
$$

$$
\text { where } \begin{aligned}
r_{0, j} & =\text { outer radius of subcylinder } j \\
r_{i, j+1} & =\text { inner radius of subcylinder } j+1 \\
j & =\text { the number of the subcylinder }
\end{aligned}
$$


- the height of all subcylinders is the same, thus

$$
h_{j}=h \quad 1 \leq j \leq n
$$

where $h_{j}=$ height of subcylinder $j$

$h=$ height of thick-walled cylinder.

- in a plane perpendicular to the axis the radial and tangential coordinates are assumed to be the principal directions of the strain rate tensor, and hence

$$
\alpha_{j}=\alpha \quad 1 \leq j \leq n
$$

where $\alpha_{j}=$ torsion angle of subcylinder $j$

$\alpha=$ torsion angle of thick-walled cylinder

- for the volume of the $j^{\text {th }}$ subcylinder it holds

$$
v_{j}=v_{m, j}+v_{c, j}
$$

$$
\text { where } \begin{aligned}
V_{m, j} & =\text { muscle volume in subcylinder } j \\
V_{c, j} & =\text { coronary volume in subcylinder } j
\end{aligned}
$$

The muscle volume is determined by the initial dimensions of the thick-walled cylinder (chapter 9).

Using equations (7.2.1), (7.2.2) and (7.2.4) the dimensions of each subcylinder can be determined from $h$ and the characteristic quantities $V_{i v}$ and $V_{C, j}$ as described in chapter 4 . The inner radius of the thick-walled cylinder $r_{i v}$ is given by

$$
r_{i v}=\sqrt{\frac{V_{l v}}{\pi h}}=r_{i, 1}
$$

Using relation (7.2.1) in successive steps, the outer radius of each subcylinder is determined by

$$
r_{0, j}=\sqrt{r_{i, j}{ }^{2}+\frac{V_{j}}{\pi h}}
$$

It should be remarked that the vaiues of $\alpha$ and $h$ are not known, since they are no characteristic quantities. In the present chapter it will be shown, that they can be found from two equations of equi]ibrium of forces and torques acting on the upper surface of the thick-walled cylinder. 


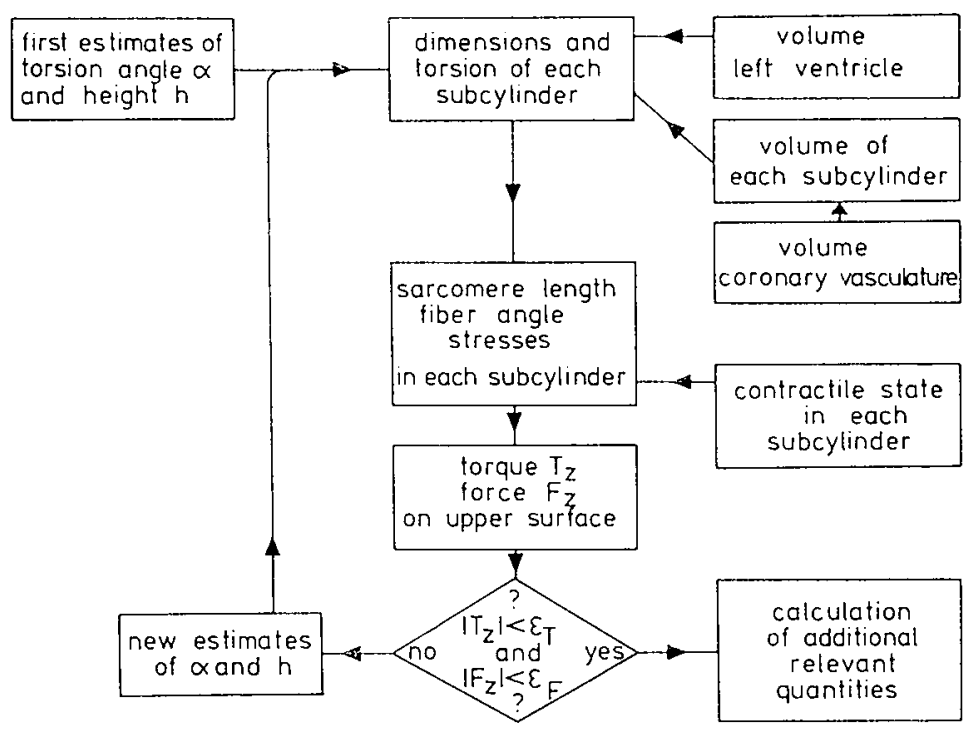

Figure 7.1.

Blockdiagram of the sequence of calculations in order to obtain the state of deformation of the thick-walled cylinder, which simulates the left ventricle. After completion of this calculation additional relevant quantities are computed.

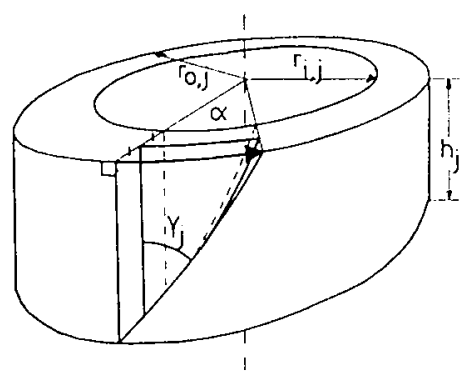

Figure 7.2.

Definition of the shear angle $\gamma_{j}$ in a subcylinder. The arrow denotes the rotation of the upper surface over an angle a due to the deformation of the subcylinder.

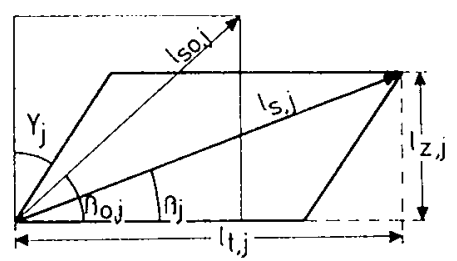

Figure 7.3 .

Deformation of a rectangular area to a parallelogram in the center plane of a subcylinder. 


\subsection{Coiluputation of the stresses in a subcylinder}

Both the dimensions of a subcylinder and the torsion angle determine the deformation of the wall material. The dimensions of subcylinder $j$ are the height $h_{j}$, the inner radius $r_{i, j}$ and the outer radius $r_{0, j}$ and are shown in fig. 7.2. The torsion angle $\mathrm{s}_{j}$ is also shown. The values of $h_{j}, r_{i, j}, r_{0, j}$ during the initial state are denoted as $H_{j}, R_{i, j}, R_{0, j}$ respectively, whereas $a_{j}=0$. The average values of the natural strain in the wall are given by

$$
\begin{aligned}
& r_{z, j}=\ln _{n}\left[\begin{array}{l}
n_{j} \\
\bar{H}_{j}
\end{array}\right] \\
& c_{t, j}=\ln \left[\frac{r_{0, j}+r_{i, j}}{R_{0, j}+R_{j, j}}\right] \\
& c_{r, j}=\ln \left[\begin{array}{l}
r_{0, j}-r_{j, j} \\
R_{0, j}-R_{i, j}
\end{array}\right]
\end{aligned}
$$

The shear angle $\gamma_{j}$ (fig. 7.2) which defines the shear deformation in the center plane is given by

$$
\tan r_{j}=\frac{a\left(r_{0, j}+r_{i, j}\right)}{2 h_{j}}
$$

For further calculations a rectangular area of the center plane of subcylinder $j$ in the initial state of deformation is considered (fig. 7.3). The sides of this rectangle are chosen to be parallel to the $z$ - and $t$-coordinates. The diagonal is parallel to the initial fiber direction and the length is equal to the initial sarcomere length $I_{s o, j}$. The angle between the initial fiber direction and the $t$-coordinate is denoted as $\beta_{0, j}$.

After deforming the subcylinder according to relations (7.3.1), (7.3.2) and (7.3.4) the rectangle is distorted to a parallelogram as shown in fig. 7.3 . For the projection of the diagonal on the $z$ - and $t$-coordinate, $l_{z, j}$ and $l_{t, j}$ respectively, it holds

$$
l_{z, j}=\frac{h_{j}}{H_{j}} l_{s o, j} \sin \beta_{0, j}
$$


$l_{t, j}=\frac{a\left(r_{0, j}+r_{i, j}\right)}{2 H_{j}} 1_{s 0, j} \sin r_{0, j}+\frac{r_{0, j}+r_{j, j}}{R_{0, j}+R_{i, j}} l_{s o, j} \cos \beta_{0, j}$

The instantaneous sarcomere lengtio equals

$$
l_{s, j}=\sqrt{1_{t, j}^{2}+r_{z, j}^{2}}
$$

and the instantaneous fiber angle $\beta_{j}$ is

$$
B_{j}=\arctan \left(\frac{1}{T_{t, j}, j}\right)
$$

Substitution of the value of $]_{s, j}$ as found from equation (7.3.7) into the equations $(5.3 .5),(5.3 .15),(5.3 .4)$ and $(5.3 .1)$ in this order, the stress ${ }_{f, j}$ on the fiber structures is found to be

$a_{f, j}=I_{s, j} \mid G_{p o}\left(\exp \left\{b\left(l_{s, j}-a\right)\right\}-1\right)$

$$
+\left(1_{s, j}-I_{s a, j}\right) \cdot \frac{G_{a o} A_{1}\left(I_{s a}\right)}{40 \cdot 10^{-9} m}
$$

A11 the other quantities in relation (7.3.9) either are characteristic quantities as mentioned in chapter 4 or they are known parameters. The stress $\sigma_{f, j}$ acts on the muscle fibers, which make an angle $\sigma_{j}$ with the cross-sectional plane of the subcylinder. Applying general laws of stress to the fiber stress $f_{f, j}$, the normal stress $v_{f z, j}$ in axial direction, the nomal stress $v_{f t, j}$ in tangential direction and the shear stress $\sigma_{f t z, j}$ in the upper surface of the subcylinder can be formulated as

$$
\begin{aligned}
& \sigma_{f t, j}=\sigma_{f, j} \cos ^{2} \beta_{j} \\
& \sigma_{f z, j}=\sigma_{f, j} \sin ^{2} \beta_{j} \\
& \sigma_{f t z, j}=\sigma_{f, j} \cos \beta_{j} \sin \beta_{j}
\end{aligned}
$$

According to the concept of anisotropic material (chapter 5) in each subcylinder a hydrostatic component $-p_{i m}$ in the soft material can be added to the nornal stresses. 
7.4. Equilibrium of force and torque acting on the upper surface of the thickwalled cylinder

As mentioned in section 2, two relations of equilibrium in the upper surface of the thick-walled cylinder are necessary. The forces acting in the axial direction on the upper surface of the thick-walled cylinder are:

- the force exerted by the left ventricular pressure and acting on the upper surface of the cavity;

- the forces due to the axial stresses in the various subcylinders;

- the force exerted by the endocardium and other structures, in the left ventricular cavity.

In the model for each subcylinder the contribution $\Delta F_{z, j}$ to the force $F_{z}$ acting on the upper surface is computed as a resultant of both the axial stress acting on the upper surface of that subcylinder and the left ventricular pressure increment as generated by the tangential stress. The latter pressure is assumed to act on the surface which corresponds to the radius of the center plane (fig. 7.4). Because the upper surface of the thick-walled cylinder is in equilibrium, the total force in axial direction on that surface $F_{z}$ must be zero. According to the definition for $\Delta F_{z, j}$ it holds

$$
\Delta F_{z, j}=\pi\left(\sigma_{f z, j}-\frac{1}{2} \sigma_{f t, j}\right)\left(r_{o, j}{ }^{2}-r_{i, j}{ }^{2}\right)
$$

where $\sigma_{f t, j}$ and $\sigma_{f z, j}$ are defined in relation to equations (7.3.10) and (7.3.11).

obviously $\Delta F_{z, j}$ is independent of externally applied hydrostatic pressure, since the subcylinders and the cavity content are incompressible. The conclusion is that the force $\Delta F_{z, j}$ only depends on the stress in the fiber structure as expressed by relation (7.4.1). Fig. 7.5 shows schematically the composition of the total axial force acting on the system of subcylinders. Each subcylinder is supposed to be surrounded by a thin film of fluid under a hydrostatic pressure, exerted by the surrounding subcylinders.

The axial forces $\Delta F_{z, j}$, necessary to keep the subcylinders in the actual state of deformation, and the axial force exerted by the endocardium and other structures in the left ventricular cavity $F_{\text {endo }}$ result in the total axial force $F_{z}$, hence

$$
F_{z}=\sum_{j=1}^{n} \Delta F_{z, j}+F_{\text {endo }}
$$




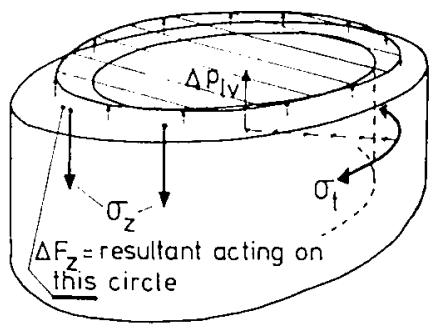

Figure 7.4 .

Schematical representation of the calculation of the force per subcylinder $\Delta \mathrm{F}_{Z}$. According to Laplace's law the tangential stress ot generates a pressure plv, which acts on the upper, shaded area. The resulting force counteracts the force acting on the upper surface of the wall of the subcylinder due to the axial stress $\sigma_{z}$. The resultant equals $\Delta F_{z}$.

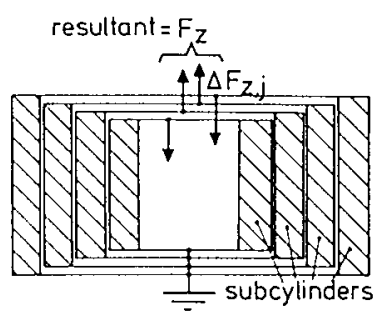

Figure 7.5 .

In a state of equilibrium the resultant $F_{Z}$ of all forces $\Delta F_{Z}$ and the axial force in the endocardium must be zero.

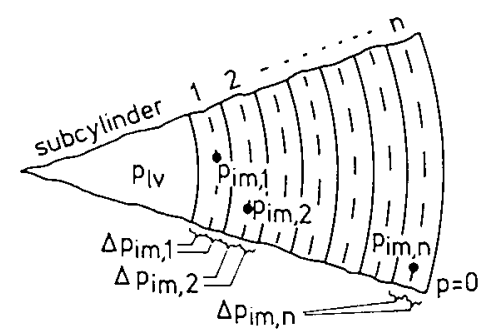

Figure 7.6.

Calculation of the intramyocardial pressures $p_{i m, j}$ and the left ventricular pressure plv by summation of pressure increments $\Delta p_{i m, j}$ starting from the epicardial side. 
The equilibrium of axial forces requires

$$
F_{z}=0
$$

In an analogeous way the equilibrium of torques in the upper surface is dealt with. Each subcylinder $j$ contributes a torque $i T_{z, j}$ to the total torque $T_{z}$ acting on the upper surface of the thick-walled cylinder. The cavity and endocardium do not contribute. For the torque contribution $\therefore T_{z, j}$ it holds

$$
M T_{z, j}=\frac{\pi}{2}\left(r_{0, j}{ }^{2}-r_{i, j}^{2}\right)\left(r_{0, j}+r_{i, j}\right) v_{f t z, j}
$$

where $\sigma_{f t z}, j$ is defined in relation to equation (7.3.12). The torque does not depend on the hydrostatic pressure. The total torque $T_{z}$ is described by

$$
T_{z}=\sum_{j=1}^{n} i T_{z, j}
$$

The equilibrium of torque in the upper surface requires

$$
T_{z}=0
$$

The quantities $F_{z}$ and $T_{z}$ finally depend on the variables $h$ and $c_{t}$, defined in section 7.2. Solution of the equations (7.4.3) and (7.4.6) results in the actual values of the height $h$ and the torsion angle $a$. In the model a second order iterative procedure is used to soive this system of two equations with two unknown variables.

7.5. Calculation of local intramyocardial pressures and left ventricular pressure

The left ventricular pressure equals the intramyocardial pressure at the endocardial surface. The intramyocardial pressure is defined as the pressure in the soft material surrounding the myocardial fibers. Since the myocardial fibers are assumed to be parallel to the myocardial wall, the radial component $\sigma_{r}$ of the stress has the opposite value of the local intramyocardial pressure $p_{i m}$

$$
p_{i m}(r)=-\sigma_{r}(r)
$$


When applying Laplace's law to the cylinder wall the tangential component of the stress ${ }_{i}$ is

$$
v_{t}=r \frac{d v_{r}}{d r}
$$

When using the model of subcylinders, relation (7.5.2) renders an approximation for the intranyocardial pressure increment $\therefore p_{i n, j}$ over one half of subcylinder $j$ :

$$
\Lambda \rho_{i m, j}=o_{f t, j} \frac{r_{0, j}-r_{i, j}}{r_{o, j}+r_{i, j}}
$$

The value of the intramyocardial pressure $p_{i m, j}$ in the center region of subcylinder $j$ is obtained by sumnation of intramyocardial pressure increments $\Delta p_{i m, j}$, beginning from the outer subcylinder, as is shown in fig. 7.6. The external pressure is supposed to be zero. The intramyocardial pressure in the outer subcylinder $p_{i m, n}$ is described by

$$
p_{i m, n}=s_{i m, n}
$$

where $\Lambda p_{i m, n}$ is obtained from equation (7.5.3). The intramyocardial pressure in each subcylinder is obtained by subsequently applying

$$
p_{i, j}=p_{i \text { [il }, \mathbf{j}+1}+A p_{i m, j+1}+\Delta p_{i m, j}
$$

starting from $j=n-1$ downwards to $j=1$. The left ventricular pressure is found by adding the pressure increment belonging to the inner half of the inner subcylinder to the corresponding intramyocardial pressure, and thus

$$
p_{1 v}=p_{i m, 1}+\Delta p_{i m, 1}
$$




\subsection{Introduction}

This chapier deals with some physiologically relevant quantities, which are not calculated in the preceding chaptcrs. In section 8.2 aortic volume flow and pressure are calculated. Horeover, the derivatives of both characteristic quantities, ventricuiar voime $\left(V_{1 V}\right)$ and arterial pressure $\left(p_{a r t}\right)$ are determined. Section 8.3 deais with the generated mechanical specific energy, which is defined as the mechanical energy generated locally in the myocardium per unit of volume. Within each subcylinder this specific energy is assumed to be constant. In section 8.4 for each subcylinder coronary perfusion is calculated. In the present study coronary perfusion is defined as the volume of blood, which flows through the capillaries per volume of tissue during one cardiac cycle.

\subsection{Calculation of aortic volume flow and aortic pressure}

The aortic volume flow $q_{a o}$ is defined as the volume flow through the aortic valve. As mentioned in chapter 3 the aortic valve is supposed to have ideal characteristics whereas the aortic input impedance in the direction of the aorta consists of the characteristic impedance $Z_{a o}$ in series with the arterial capacitance $C_{a r t}$, which is in parallel with the peripheral resistance $R_{p}$ (fig. 3.2). The impedance $z_{\text {ao }}$ is purely resistive. The arterial pressure $p_{a r t}$ is defined as the pressure over the capacitance $C_{\text {art }}$ and is a characteristic quantity of the system (chapter 4 ). The aortic volume flow $q_{a 0}$ is determined by $p_{l v}$ and $p_{a r t}$ :

$$
\begin{aligned}
& \text { if } p_{1 v}<p_{\text {art }} \text { then } q_{a 0}=0 \\
& \text { if } p_{1 v}>p_{a r t} \text { then } q_{a_{0}}=\frac{p_{1 v}-p_{a r t}}{Z_{a o}}
\end{aligned}
$$

The aortic pressure $p_{\text {ao }}$ distal to the aortic valve follows from

$$
p_{\text {ao }}=p_{\text {art }}+z_{\text {ao }} q_{a o}
$$

The derivative of the volume of the arterial capacitance equals the differ- 
ence between the aortic volume flow and the volume flow through the peripheral resistance. Hence, the derivative of the arterial pressure is determined by

$$
\frac{d p a r t}{d t}=\frac{1}{c} \frac{1}{a r t}\left(q_{a 0}-\frac{p_{a r t}}{R_{p}}\right)
$$

The derivative of ventricular volume is calculated through

$$
\frac{d v_{l v}}{d t}=q_{1 v, \text { in }}-q_{a 0}
$$

where $q_{l_{v}, \text { in }}=$ inflow of the left ventricle (nitral volume flow). Juring the first half of the cardiac cycle (systole; $0<t<\frac{1}{2} t_{c y c l}$; it is assumed that

$$
q_{7 v, \text { in }}=0
$$

and during the second half (diastole; $\frac{1}{2} t_{c y c l}<t<t_{c y c l}$ ):

$$
q_{\text {Tv, in }}=\frac{2 v_{e j}}{t_{c y c l}}
$$

where $v_{e j}=$ volume, which is ejected from the left ventricle into the aorta during the preceding systole

$$
t_{\text {cycl }}=\text { duration of the cardiac cycle. }
$$

8.3. Calculation of the mechanical specific energy, generated during the cardiac cycle

The heart muscle generates energy which is necessary for the ejection of blood into the aorta. This energy is the sum of the energy produced by the separate subcylinders. Since mechanical loading in each subcylinder is different, transmural differences in generated mechanical energy per unit of muscle volume per beat $\left(w_{j}\right)$ exist. This generated mechanical specific energy $\left(w_{j}\right)$ is especially interesting with respect to local coronary perfusion, which is also calculated by the present model. A necessary condition for normal cardiac performance is a sufficiently large value of the ratio of coronary perfusion and produced mechanical specific energy in all parts of the myocardium. In each 
subcylinder ( $j)$ the instantaneous values of the sarcomere length $\left(1_{s, j}\right)$ and the force per sarcomere volume $\left(G_{j}\right)$ are calculated. For the generated mechanical specific power it holds

$$
\frac{d{ }^{j}}{d t}=-G_{j} \frac{d 1 s, j}{d t}
$$

Integration of equation (8.3.1) over one cardiac cycle renders

$$
w_{j}=-\int_{t=0}^{t=t}{ }^{c y c)} G_{j}(t) d l_{s, j}(t)
$$

The integral (8.3.2) is evaluated numerically to a second order of accuracy. As a test for the accuracy of the calculation in the model the total energy $\left(H_{m}\right)$, which is generated by the cardiac muscle material is calculated. It holds that

$$
H_{\mathrm{III}}=\sum_{j=1}^{n} w_{j} V_{m, j}
$$

where $v_{m, j}=$ muscle volume in subcylinder $j$.

The energy per beat (stroke work) of the left ventricle $\left(\omega_{s}\right)$ is computed numerically according to

$$
H_{s}=-\int_{t=0}^{t=t_{c y c l}} p_{1 v}\left(\frac{d v / v}{d t}\right) d t
$$

where $d v_{7 v^{\prime}} / d t$ is calculated according to relation (3.2.4). The energy loss $\left(W_{C}\right)$ due to squeezing of the coronary circulation is determined by intramyocardial pressure and changes of the coronary vascular volume and is calculated according to

$$
w_{c}=-\int_{t=0}^{t=t}{ }^{t y c l} \sum_{j=1}^{n} p_{i m, j} \frac{d\left(v_{c a, j}+v_{c c, j}+v_{c v, j}\right)}{d t} d t
$$

Since $\left|W_{c}\right| \ll\left|W_{m}\right|$ the energy $W_{c}$ is approximated by the sum of the products of the mean coronary volume change and mean intramyocardial pressure in each subcylinder during systole. If all calculations are correct, due to the principle 
af conservation of energy it must hold

$$
\left\|_{\mathrm{s}}:\right\|_{c}-H_{\mathrm{m}}
$$

\section{A. Calculation of the Tocal coronary perfusion fraction in one cardiac cycle}

In section 8.3 the relevance of the calculation of the local coronary perfusion $\left(f_{j}\right)$ is indicated. The perfusion $\left(f_{j}\right)$ in each subcylinder is defined as the amount of blood, wich flows through a unit of volume of tissue during one cardiac cycle. Obviously, this quantity is dimensionless. Under stationary conditions the volume flow through each subcompartment of the microcirculation within one subcylinder is the same. In the present model the inflow of the capillaries is chosen to be representative for the volume flow through the licrocirculatory compartment. Thus for $f_{j}$ it holds

$$
f_{j}=\int_{t=0}^{t=t} \int_{c y c]}^{q_{a c, j}} d t
$$

The inflow $\left(q_{a c, j}\right)$ of the capillary subcompartment in subcylinder $j$ is calculated through relation (6.4.7). The integral $(8.4 .1)$ is calculated nunierically to a second order of accuracy. 
9. INPUT DATA ANO INITIAL CONDITIONS OF THE PRESEHT HODËL

\subsection{Introduction}

The present model simulates the dynamics of the left ventricie and the coronary circulation under various experimental hemodynamic conditions. The measurable quantities of the left ventricle and the hemodymamics should be the same in the mode? and in the experimental situation. Hence many quantitative data obtained in the experimenta? situation are used in the model. These data are the parameters of the relations which foril the mathematical model (chapter 1). Many of these parameters are taken either from animal experiments reported in literature or obtained from own investigations. In this way, however, some parameters could not be cetermined accurately and, thence, these parameters have to be estimated by comparing the results obtained from the model and those obtained from animal experiments.

Section 9.2 deals with the input data concerning initial, end-diastolic, values of the following quantities: geometry of the left ventricle, initial sarcomere length, sequence of depolarization, cardiovascular hemodynamics and finally initial volume, capacitance and resistance of the different subcompartments in the coronary circulation. In chapter 4 the present model is described by a system of differential equations. In order to be able to solve this system, at a given time $t_{0}$, all values of the characteristic quantities must be known. In section 9.3 these initial conditions of the system of differential equations are discussed.

\subsection{Input data of the present mode?}

\subsubsection{The geometry of the left ventricle}

During the cardiac cycle the geonetry of the left ventricle is changing. Characterization of this geometry at any particular moment, however, is sufficient to define the related initial conditions of the model. In the present model the normal end-diastolic state of the left ventricle is chosen as a reference since about this state most information is available. In chapter 6 experiments on dogs with an average heartweight of $230 \mathrm{~g}$ are described. Since scaling rules of the coronary circulation are not exactiy known, this heartweight rather arbitrarely is chosen as reference. In the model only the left ventricle is simulated. Considering the pressure relation, the pumping power of the left ventricle is normally 5 times the pumping power of the right ven- 
tricle. Assuming that pumping power of a ventricle corresponds with the effective weight of that ventricle, the effective weight of the left ventricle $\left(G_{1 v}\right)$ is $5 / 6$ of the total weight of the ventricles. Knowing that the weight of the ventricles is 90: of the heartweight (own observation on 2 dogs), it follows that

$$
G_{\text {IV }}=172.5 \mathrm{~g}
$$

An important paraneter conceming the geonetry of the left ventricle is the end-diastolic ratio of the inner radius $\left(R_{i v}\right)$ and outer radius $\left(R_{\text {ov }}\right)$ in the equatorial plane. Ross et al. ${ }^{18)}$ determined both radi from direct measurements on post mortem hearts which were arrested at end-diastole. The ratio $R_{i v} / R_{0 v}$ was found to be 0.67 . Other investigators $1,25,31$ ) determined the inner diameter and wall thickness of the left ventricle by means of ultrasound. From their results it is concluded that the values of the end-diastolic ratio of $R_{j y} / R_{\text {ov }}$ ranges in 0.65 to 0.75 . From angiografic studies by Sandler et al. 21 , $22)$ and Liedtke et a $1{ }^{12)}$ values between 0.63 and 0.77 are derived. Direct determination of the dimensions of the heart, nowever, is not very accurate since the endocardial surface is irregular and not well defined. lloreover, the definition of a normal end-diastolic state is subject to arbitrariness. These disadvantages are also present in angiografic and ultrasonic measurements.

Another method to determine the ratio of outer to imer radius is based on the presumed cylindrical geometry of the left ventricle. Then it holds that

$$
\left(\frac{R_{i v}}{R_{o v}}\right)^{2}=\frac{v_{l v, e d}}{v_{\text {lv,ed }}+v_{\text {lvw }}}
$$

where $V_{\text {Iv, ed }}=$ end-diastolic left ventricular cavity volume $V_{l v}=$ volume of the left ventricular wall. Hanna ${ }^{7}$, Ross et al. ${ }^{18)}$ and Scott-Rankin et al. ${ }^{25)}$ determined both the enddiastolic left ventricle cavity volume and the weight of either the heart or the left ventricle. Assuming a myocardial density of $1050 \mathrm{~kg} \mathrm{~m}^{-3}$ relation (9.2.2) is applied to determine the ratio $R_{i v} / R_{\text {ov }}$. In this way values of 0.62 , 0.61 and 0.65 were found for this ratio by the different investigators. Probably because of the presumed cylindrical geometry, the latter ratios are somewhat less than the values as mentioned before. Taking into account that the latter method seems most appropriate, as a compromise between the different values of the ratio as taken from literature is chosen 


$$
\frac{R_{\text {iv }}}{R_{\text {OV }}}=0.65
$$

To determine the initial height of the cylinder $\left(H_{v}\right)$ another parameter is introduced. This parameter is defined by the end-diastolic ratio

$$
b_{e d}=\frac{H_{v}}{R_{i v}}+\frac{v}{R_{o v}}
$$

This ratio is calculated by

$$
b_{e d}=\frac{V_{l v w}}{\pi\left(R_{i v}+R_{o v}\right)\left(R_{o v}{ }^{2}-R_{i v}{ }^{2}\right)}
$$

In the derivation of relation (9.2.5) a cylindrical geometry of the left ventricle is assumed. According to this relation bed is deternined to be equal to 1.22 and 1.51 in the studies reported by Scott-Rankin et al. ${ }^{25}$ ) and McHale et a1. ${ }^{15)}$. As a compromise between these values is chosen

$$
b_{\text {ed }}=1.35
$$

Combination of the equations $(9.2 .6),(9.2 .5),(9.2 .4),(9.2 .3)$ and $(9.2 .1)$ when taking the myocardial density to be $1050 \mathrm{~kg} \mathrm{~m}^{-3}$ renders

$$
\begin{aligned}
& R_{i v}=22.4 \mathrm{~mm} \\
& R_{\text {OV }}=34.4 \mathrm{~mm} \\
& H_{V}=76.6 \mathrm{~mm}
\end{aligned}
$$

by which quantities the geometry of the cylinder is characterized at end-diastole.

To characterize transmural differences sufficiently accurate the number of subcylinders is chosen to be

$$
n=8
$$

In this case errors as a consequence of the presumed thin-walled behavior of the subcylinder are negligible $(<0.1 \%)$. 
9.2.2. The fiber orientation across the nyocardial walI

Hore than three hundred years ago Lower ${ }^{13}$ ) found that the myocardial fibers run parallel mainly to the epicardium and moreover that the orientation of those fibers changes across the myocardial wall. In the center layers of the wall, the fibers are approximately parallel to the equator, whereas in the subepicardium and subendocardium the fibers are more axially directed (fig. 3.1 ). The fiber structure in relation with the geometry of the heart is described more in detail by Grant ${ }^{5)}$, Robb et al. ${ }^{17}$ ) and Rushmer et al. 20).

Quantitative data about the change in fiber orientation across the myocardiat wall are presented by Hort ${ }^{10)}$, Ross et al. ${ }^{19)}$ and Streeter et al. ${ }^{29,30)}$. In these studies the fiber angle is measured as a function of the distance from the endocardium. The fiber angle is defined as the angle between the fiber direction and the parallel of latitude (fig. 9.1). Since the present model simulates the equatorial region of the left ventricle (chapter 3 ), data on the left ventricular free wall in the equatorial region are used.

Figure 9.2 shows data, obtained by several authors $10,19,30)$ from different animals. Streeter et al. ${ }^{30)}$ measured the fiber angle in the equatorial region of the left ventricular free wall of a canine heart, which was arrested in the end-diastolic phase. Hort ${ }^{10}$ ) measured the fiber angle across the wall in a canine heart in rigor as well as in a dilated state. In these two cases the course of the fiber angle was different, which is explained by changed geometry of the heart. Hort ${ }^{10)}$ also reported that in a guinea pig heart the fiber angle in the subendocardial region is significantly larger as compared to other species. Ross et al. 19) found that the course of the fiber angle in a macaque heart is not significantly different from that in a canine heart.

In the present model of the left ventricle the data of Streeter et al. 30) are used because his study is best detailed. Moreover, he specified the fiber angle in the end-diastolic state which is the initial state of the left ventricle in the present model.

\subsubsection{Ihe sarcomere length across the myocardial wall}

As mentioned before (section 9.2.1) the normal end-diastolic state of the left ventricle is chosen as a reference. In this particular state the distribution of the sarcomere length across the myocardial wall must be known because stress generated by cardiac muscle strongly depends on the sarcomere length (chapter 5).

Several investigators $10,28,34$ ) have measured transmural differences in sar- 


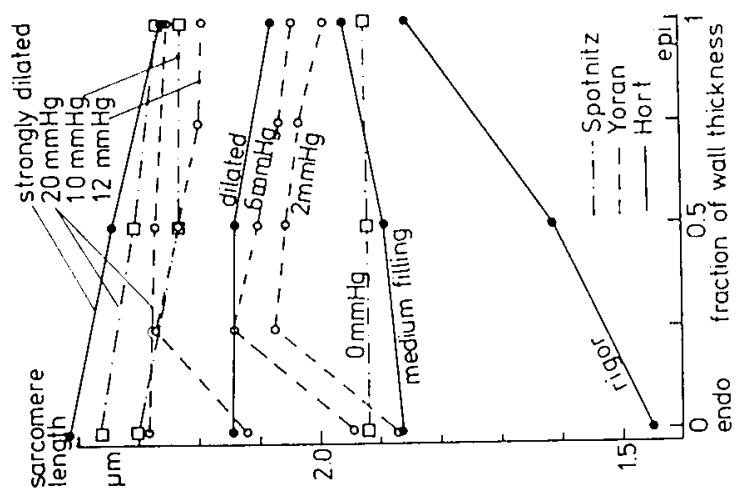

导点 号

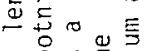

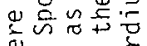

Eलि

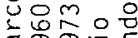

in

$\div \div \div \frac{\alpha}{\sigma}$

온 m. の \& इ

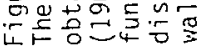
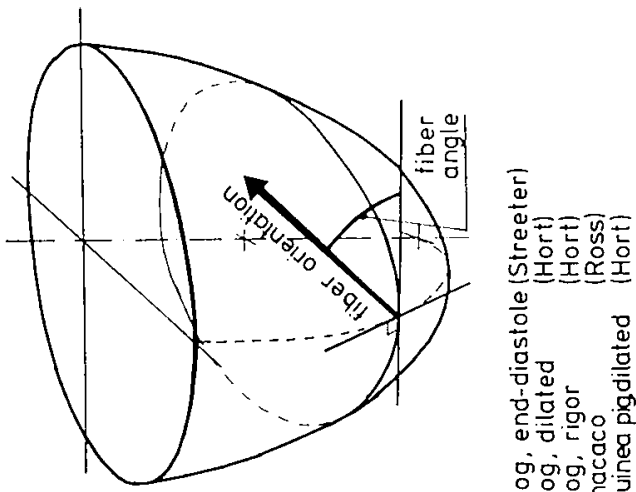

in 4

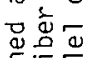

둔

施 융용

岁昰竞

$\sim$ 兄

$\stackrel{\alpha}{\circ}$

. 究㟔总

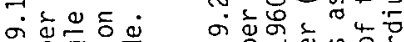

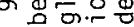

ㄴㄷㄴㄷㅠ

要造

돈

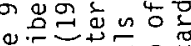

2

元4

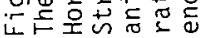

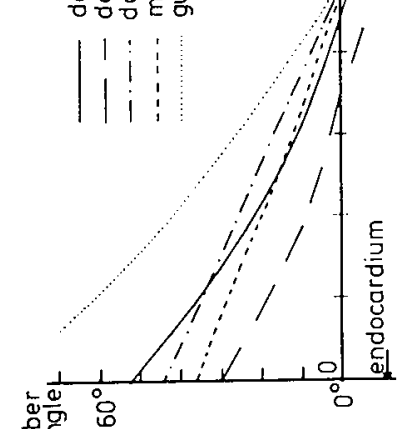


comere length across the myocardial wall under various filling conditions. Fig. 9.3 shows these data grafically. In experiments on dogs Yoran et al. ${ }^{34}$ ) determined the sarcomere length in five layers of the left ventricular free wall at left ventricular end-diastolic pressures of 2, 6, 12 and $20 \mathrm{mmH}$. At the lower pressures the subendocardial sarcomere length was significantly less than the sarcomere length in the midwall region. In a similar study Spotnizt et al. 28) considered three layers. Fron his data Wong et al. 33) derived the sarcomere length in these three layers for various left ventricular enddiastolic pressures. Sonnenblick et al. ${ }^{27)}$ reported sarcomere lengths of 2.07 lain and $2.25 \mathrm{~mm}$ in the midwall region at diastolic pressures of $8 \mathrm{mmHg}$ and 32 mullig, respectively. These data agree fairly well with those obtained by other investigators (fig. 9.3).

In the present model of the left ventricle the data of Yoran et al. ${ }^{34}$ ) are used, since his study is most detailed. It is assumed, that the normal enddiastolic pressure is approximately $6 \mathrm{mmHg}$.

\subsection{Velocity of the depolarization wave front across the myocardial wal]}

In chapter 3 the spread of activation across the myocardial wall is assumed to be purely radial. Several investigators $3,4,23,24,26)$ measured the onset of electrical activity at different sites in the myocardium. Durrer et al. $\left.{ }^{3}, 4\right)$ reported a velocity of approximately $0.4 \mathrm{~m} \mathrm{~s}^{-1}$ for the velocity of the depolarization wave front across the left ventricular wa 11 . In isolated hearts the same authors reported values of approximately $5.5 \mathrm{~m} \mathrm{~s}^{-1}$, which value is significantly higher than in the heart in situ. This discrepancy may be partly due to shrinkage of the left ventricular cavity in the Langendorf perfusion system used, so that the ventricular wall might have thickened considerably. Assuming a constant travelling time of the depolarization wave across the wall a higher value of the velocity will be found. In intact dogs Scher et al. 23,24$)$ reported a value of $0.4 \mathrm{~m} \mathrm{~s}^{-1}$ for this velocity, which agrees with the findings of Durrer ${ }^{3,4)}$. In the present model this value of $0.4 \mathrm{~m} \mathrm{~s}^{-1}$ is used.

\subsubsection{The input_impedance of the_aorta}

The input impedance of the aorta is represented by a network ${ }^{32}$ ) consisting of the characteristic impedance of the aorta $\left(Z_{a_{0}}\right)$ in series with the peripheral resistance $\left(R_{p}\right)$, which is in parallel with the arterial capacitance ( $C_{\text {art }}$; fig. 3.2). In different dogs the values of these parameters can vary 
tremendously. 0lder dogs have a stiffer vasculature, resulting in a lower value of $C_{\text {art }}$ and a higher value of $z_{a o}$. Because smaller dogs have a smaller aorta and a lower aortic volume flow, $C_{a r t}$ is lower and $Z_{\text {ao }}$ and $R_{p}$ are higher than in larger dogs. Therefore, when simulating an animal experiment by the present model, the values of these parameters must be taken from that particular experiment. As to this both aortic pressure $p_{\text {ao }}$ and aortic volume flow $\mathrm{q}_{\mathrm{a} o}$ just distal to the aortic valve are recorded simultaneously. The sum of both resistances $Z_{\text {ao }}$ and $R_{p}$ is

$$
z_{a 0}+R_{p}=\frac{\overline{p_{a o}}}{\overline{q_{a o}}}
$$

where $\overline{p_{a 0}}$ and $\overline{q_{a 0}}$ are mean aortic pressure and volume flow, respectively. After closure of the aortic valve at end-systole the aortic pressure decreases exponentially until next beat occurs. For the related time constant $\tau_{\text {ao }}$ it holds

$$
\tau_{a o}=R_{p} C_{a r t}
$$

For the higher harmonics $(>5 \mathrm{~Hz}$ ) in the pressure and flow signals the influence of the peripheral resistance is small, and the input impedance approaches the characteristic impedance of the aorta. A sufficiently accurate method for the calculation of $z_{a 0}$ is

$$
z_{\mathrm{ao}}=\frac{\int_{\substack{\text { systole } \\ \text { systole }}}\left(p_{\mathrm{ao}}-\overline{\mathrm{p}_{\mathrm{ao}, \mathrm{d}}}\right) d t}{\mathrm{q}_{\mathrm{ao}} \mathrm{dt}}
$$

where $p_{a 0, d}=$ mean diastolic aortic pressure. Fig. 9.4 elucidates relation $(9.2 .11)$. Combination of the relations $(9.2 .9),(9.2 .10)$ and $(9.2 .11)$ renders the values of $Z_{a 0}, R_{p}$ and $C_{a r t}$. In the present model the following values are used

$$
\begin{aligned}
& R_{p}=1.1 \cdot 10^{8} \mathrm{~N} \mathrm{~m}^{-5} \\
& Z_{\text {ao }}=1.7 \cdot 10^{7} \mathrm{~N} \mathrm{~m}^{-5} \mathrm{~s} \\
& C_{\text {art }}=3.0 \cdot 10^{-8} \mathrm{~N}^{-1} \mathrm{~m}^{5}
\end{aligned}
$$


Figure 9.4.

Graphical representation of the calculation of the aortic characteristic impedance from the recordings of aortic pressure and aortic volume flow.

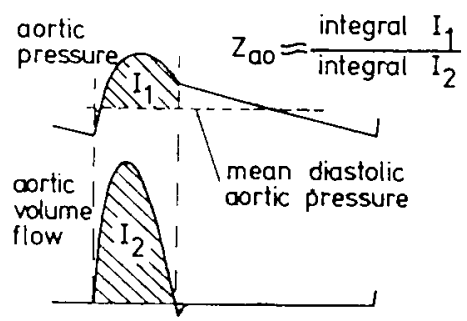

These data are based on an experiment on a dog of $25 \mathrm{~kg}$ and extrapolated to the relatively large heartweight in the present model. In this extrapolation aortic volume flow is assumed to be proportional to the heartweight, and aortic pressure to be unaffected.

\subsubsection{Hemodynamic guantities}

In order to define the hemodynamic loading of the left ventricle, end-diastolic values of some hemodynamic quantities belong to the input data. These quantities are:

- the end-diastolic aortic pressure determining the moment of opening of the aortic valve at the beginning of the ejection phase.

- the end-diastolic volume of the left ventricular cavity determining the enddiastolic left ventricular pressure and geometry. In the present model mitral inflow is calculated so that the end-diastolic left ventricular volume is again reached at the end of the cardiac cycle. The mitral volume flow is assumed to be zero during the first half (systole) of the cardiac cycle and taken constant during the second half (diastole).

- the heart rate determining the duration of the cardiac cycle.

- the pressure in the right atrium, which is assumed to be constant and wich is relevant for the coronary circulation.

In general at the end of the simulated cardiac cycle, the aortic pressure is not necessarely equal to the initial end-diastolic value of that pressure. After several beats the end-diastolic aortic pressure reaches a stationary Ievel, which is independent of the initial value of that pressure. By choosing this pressure closer to the stationary level, steady state is reached earifier.

\subsubsection{The endocardium and other structures in the left ventricular cavity}

In the present model the endocardium and other structures in the left ven- 
tricular cavity can bear forces only in the axial direction. As already mentioned in chapter 3 , it is likely that axial forces in these structures are present because of conditions of equitibrium between axial and tangential stresses in the wall. Quantitatively this equilibrium is expressed by relation (7.4.3). Since end-diastolic fiber orientation and sarcomere length are known (section 9.2.2 and 9.2.3), the stresses in the wall can be calculated on the basis of the myocardial properties presented in chapter 5 . Using relation (7.4.2) the force $F_{\text {endo, }}$ borne by the endocardium and other structures in the left ventricular cavity, is computed for the state of reference, which is the end of diastole under normal circumstances. In the present model it is assumed that the elastic element, which is related to $F_{\text {endo }}$ has the sane elastic properties as the passive myocardial muscle. Thus it holds

$$
F_{\text {endo }}=F_{e} \exp \left[b \cdot 2.1, \exp \left(c_{z}\right)\right]
$$

where $F_{\mathrm{e}}=$ proportionality factor

$\mathrm{b}=$ parameter, mentioned in relation to the passive muscle properties $\varepsilon_{z}=$ natural strain in the axial direction

The length $2.1 \mathrm{~km}$ is supposed to be the sarcomere length, which corresponds with the endocardial, end-diastolic strain. The parameter $F_{e}$ is equal to $F_{\text {endo }}$ in the state of reference. For simplicity it is assumed that the endocardium has no slack length.

\subsubsection{Properties of the large coronary arteries (I_D. $\geq 400$ 피)}

In section 6.2 the physical properties of the large coronary arteries are discussed. The results were obtained from hearts weighing $230 \mathrm{~g}$. The total volume and capacitance of the large coronary arteries is the sum of the volumes and capacitances of the separate coronary arterial trees. The total resistance and inertance are calculated as the resistances and inertances of the trees in parallel. However, $5 / 6$ of the heart is assumed to belong to the left ventricle and hence, in the model, the volume $\left(V_{c e}\right)$ and capacitance $\left(C_{c e}\right)$ of the large coronary arteries are proportionally lower and the inertance ( $\left.L_{c e}\right)$ and resistance $\left(R_{c e}\right)$ are proportionally higher, which results in

$$
\begin{aligned}
& V_{\text {ce }}=1.33 \mathrm{ml} \\
& C_{c e}=1.4 \cdot 10^{11} \mathrm{~N}^{-1} \mathrm{~m}^{5} \\
& R_{c e}=5.7 \cdot 10^{8} \mathrm{~N} \mathrm{~s} \mathrm{~m}^{-5} \\
& L_{c e}=7.6 \cdot 10^{6} \mathrm{~N} \mathrm{~s}^{2} \mathrm{~m}^{-5}
\end{aligned}
$$


These data are based on an average pressure of $1.33 \cdot 10^{4} \mathrm{~N} \mathrm{~m}^{-2}(=100 \mathrm{mmHg})$ in the large coronary arteries.

\subsubsection{Properties of the coronary microcirculation}

Data on physical properties of the coronary microcirculation are scarce and not very reliable. Therefore, part of the related parameters has to be estimated with the use of the present model itself. Obviously this aspect of the present model can considerably be improved. However, qualitative changes in the behavior of the coronary perfusion as a result of hemodynamic changes can be studied fairly well.

A normal value for the coronary perfusion $\left.{ }^{9}\right)$ is $0.01 \mathrm{~s}^{-1}$, which means that $0.01 \mathrm{ml}$ of blood flows through $1 \mathrm{ml}$ of myocardial tissue during 1 second. Normal values for the intravascular pressures in the subepicardial layers are assumed to be the same as in skeletal muscle. In these structures Bohlen et al. 2) found for the mean pressures:

- in the aorta

- just proximal to the capillaries

- just distal to the capillaries

- in the right atrium (estimate)
$11700 \mathrm{Nm}^{-2}$ (= $\left.=88 \mathrm{mmHg}\right)$

$3600 \mathrm{~N} \mathrm{~m}^{-2}(=27 \mathrm{mmHg})$

$2100 \mathrm{~N} \mathrm{~m}^{-2}(=16 \mathrm{mmHg})$

$800 \mathrm{Nm}^{-2}(=6 \mathrm{mmHg})$

With these data the flow conductance per unit of volume is calculated as the ratio of perfusion flow ( $\mathrm{ml}$ blood per second per $\mathrm{ml}$ tissue; $\mathrm{s}^{-1}$ ) and pressure difference over the subcompartment. In this way the conductance per tissue volume of the

- arteriolar vasculature $S_{c a o}=1.23 \cdot 10^{-6} \mathrm{~N}^{-1} \mathrm{~m}^{2} \mathrm{~s}^{-1}$

- capillary vasculature $S_{c c o}=6.67 \cdot 10^{-6} \mathrm{~N}^{-1} \mathrm{~m}^{2} \mathrm{~s}^{-1}$

- venular vasculature $S_{C v o}=7.69 \cdot 10^{-6} \mathrm{~N}^{-1} \mathrm{~m}^{2} \mathrm{~s}^{-1}$

The volume $V_{c c, j}$ of the capillary subcompartment in subcylinder $j$ is calculated by

$V_{c c, j}=V_{m, j} \cdot$ capillary density $\cdot$ capillary cross-sectional area

where $v_{m, j}=$ volume of the myocardium in subcylinder $j$.

The capillary density ${ }^{1)}$ is assumed to be 3500 capillaries per $\mathrm{mm}^{2}$ and the capillary diameter is found to be $4.1 \mathrm{~mm}^{8}$ ) under normal physiological circum- 
stances. Substituting these data into relation $(9.2 .17)$ gives

$$
V_{c c, j}=0.046 V_{m, j}
$$

Reliable data on arteriolar volume $V_{c a, j}$ and venular volume $V_{c v, j}$ are not known. From histological studies ${ }^{6}$ ) it is reported that in a large part of the coronary circulation arteries and veins run in antiparallel. So the lengths of the branches in the arterial and venous coronary vascular tree are the same, but not their diameters. From relation (9.2.15) it follows that the ratio of arteriolar and venular pressure drow is approximately 6 . Hence, when assuming Poisseuille flow in the vessels, it holds

$$
\frac{V_{c V, j}}{V_{c a, j}}=\sqrt{6}=2.4
$$

In the present model it is assumed that

$$
\begin{aligned}
& v_{c a, j}=0.021 v_{m, j} \\
& v_{c v, j}=0.050 v_{m, j}
\end{aligned}
$$

These data are rough estimates, based on vascular trees of bat wing tissue ${ }^{14}$ ) and corrected for capillary density, which is larger in the heart. Inaccuracies in the estimates of these volumes have consequences for the myocardial mechanical energy dissipated in the coronary circulation as well as for the degree of compression of the subendocardial coronary vasculature during systole. For an accurate description of the behavior of the coronary nicrocirculation more attention should be paid to these paraneters.

From relation (9.2.15) the mean pressures in the center of the arteriolar, capillary and venutar subcompartments are found to be

$$
\begin{aligned}
& p_{\mathrm{Ca}}=7600 \mathrm{~N} \mathrm{~m}^{-2} \\
& p_{\mathrm{CC}}=2900 \mathrm{~N} \mathrm{~m}^{-2} \\
& p_{\mathrm{CV}}=1400 \mathrm{~N} \mathrm{~m}^{-2}
\end{aligned}
$$

The cross-sectional stiffness $K$ of a coronary vessel is assumed to be proportional to the transmural pressure. In section 6.2 for the $L A D C$ at a transmural pressure of $1.33 \cdot 10^{4} \mathrm{~N} \mathrm{~m}^{-2}(=100 \mathrm{mmHg})$ was found

$$
\mathrm{K}=9.7 \cdot 10^{4} \mathrm{~N} \mathrm{~m}^{-2}
$$


This implies, that for all subcompartments the parameters $s_{c}$ in relation $(6.3 .2)$ is

$$
s_{C}=7.2
$$

Yet, in the end-diastolic state of reference the microvascular parameters can be computed. The volume of each compartment is computed by the relations $(9.2 .18)$ and $(9.2 .20)$. Substitution of: - the transmural pressures from the relation $(9.2 .21)$, - the volumes just computed and - the parameter ${ }^{5} \mathrm{c}$ from relation (9.2.23) into relation (6.3.2) gives a value for the parameter $s_{p}$ in each subcompartment. The value of the parameter $S_{r}$ in each subcompartment is determined from the relations $(9.2 .16)$ and $(6.3 .3)$ and the above mentioned volume.

The vasoconstrictive pressure, which is defined in section 3.5 .3 , is not known since it cannot be measured. If the arterioles are completely dilated, the vasoconstrictive pressure is assumed to be zero. In that case the coronary resistance is approximately 3 times lower than in the control situation, as demonstrated by coronary reactive hyperemia ${ }^{16}$ ). Assuming that in that case the arteriolar resistance equals $25 \%$ of the normal value, the volume of the arteriolar compartment is twice the normal volume. In the present model complete vasodilation is assumed. This special situation is the most interesting one since it illustrates maximum flow capacity of the coronary circulation.

\subsection{Initial conditions}

At the start of a simulated heart cycle the values of all characteristic quantities have to be known in order to be able to solve the system of differential equations (4.1.1). The values of most of the characteristic quantities at the beginning of a heart cycle are determined by the previous heart cycle. since under normal cicumstances each heart beat is approximately identical, the condition is made that at the beginning as well as at the end of a heart cycle the characteristic quantities must have the same value. In mathematical terms this means, that the stationary solution of the system of differential equations $(4.1 .1)$ is to be found. A method to find the stationary solution is estimation of the initial values, and next solving of the system of differential equations over several cardiac cycles until the separate cardiac cycles are sufficiently identical. This process can be shortened considerabiy by accurate estimation of the initial values. 
In the present model a number of these estimates are made by a quasi-diastolic phase, which preceeds the first heart beat, and starts at $t=t_{q d}$. During this period non-stationary phenomena with a small time constant vanish. After this period the heart cycle starts at a moment defined by $t=0$. Yet, the particular characteristic quantities, of which the values are known at $t=0$, are set to these initial values. Next, as a result of solving the system of differential equations, the model simulates a heart beat. The states of the model at $t=0$ and at the end of the cycle are tested on their identity. If this identity is not sufficient, another beat is generated. The present section deals with the initial conditions of all characteristic quantities.

\subsubsection{The hemodynamics of the left ventricle and aorta}

The volume $\left(V_{1 v, e d}\right)$ of the left ventricle at $t=0$ is one of the input data. During the quasi-diastolic period the volume of the left ventricle $\left(V_{1 v}\right)$ is kept constant on this value, which requires

$$
\begin{aligned}
& \text { if } t=t_{q d} \quad \text { then } v_{1 v}=V_{T v, \text { ed }} \\
& \text { if } \quad t_{q d} \leq t \leq 0 \text { then } \frac{d v_{1 v}}{d t}=0
\end{aligned}
$$

The arterial pressure $\left(p_{\text {art }}\right)$ at the end of diastole is equal to the end-diastolic aortic pressure $\left(p_{a o, e d}\right)$ which is also one of the input data. During the quasi-diastolic phase it holds

$$
\begin{aligned}
& \text { if } t=t_{q d} \quad \text { then } p_{\text {art }}=p_{a o, e d} \\
& \text { if } \quad t_{q d} \leq t \leq 0 \text { then } \frac{d p_{a r t}}{d t}=0
\end{aligned}
$$

In a stationary situation the end-diastolic aortic pressure must be the same in each heart cycle. The initial end-diastolic aortic pressure is an estimate. The more accurate this estimate, the earlier a stationary situation is reached and the less heart beats have to be computed.

\subsubsection{Muscle mechanics}

- In chapter 5 the contractile behavior of myocardial material is characterized by two differential equations which involve two related characteristic quantities. At the beginning of the quasi-diastolic period the value of the internal sarcomere length $\left(l_{s a, j}\right)$ is made equal to the initial value of the 
sarcomere length $\left(T_{s}\right)$, which is one of the input data (section 9.2.3). The initial value of the delay $\tau_{j}$ is derived from $l_{s a, j}$ using equation (5.3.20). At the beginning of the quasi-diastolic period the geometry of the cylinder changes as a consequence of setting of the equilibria of axial force (relation 7.4.3) and torque (relation 7.4.5). During the quasi-diastiolic period the muscle is not activated and, hence, the dimensions of the cylinder are determined only by the passive characteristics of the myocardial material. At $t=0$ the value of $l_{s a, j}$ is made equal to the instanteous value of the sarcomere length. The value of the delay $\tau_{j}$ at $t=0$ is derived from $1_{s a, j}$ using equation $(5.3 .29)$.

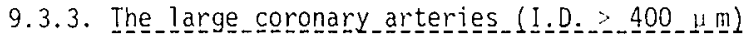

In section 6.2 it is discussed that the behavior of the large coronary arteries can be described by two characteristic quantities. The values of these quantities at the beginning of the quasi-diastolic period are roughly estimated. The initial volume flow $\left(q_{c e}\right)$ into the coronary arteries is assumed to be equal to the total flow into the microcirculatory compartment, which is estimated from a stationary, constant perfusion pressure. The volume of the coronary arteries ( $V_{c e}$ ) is determined by the pressure in the coronary arteries, which is approximately equal to the aortic pressure. Therefore, the initial value of $V_{c e}$ is based on a coronary pressure $\left(p_{c e}\right)$ equal to the aortic pressure, using relation (6.4.2). At $t=0$ these characteristic quantities are stabilized and hence, an intervention is not nevessary.

\subsubsection{The coronary microcirculation}

Section 9.2 .9 deals with the static properties of the microcirculatory compartment. It is assumed that mean aortic pressure equals $1.17 \cdot 10^{4} \mathrm{~N} \mathrm{~m}^{-2}$ (88 $\mathrm{mmHg}$ ). In the present model the initial pressures in the arteriolar, capillary and venular compartment are proportional to the initial, end-diastolic, aortic pressure $\left(p_{a o, e d}\right)$, which belongs to the input data. The ratio of the pressure drops over the arteriolar, capillary and venular compartment are presented in relation (9.2.15). According to relation (6.3.2) the initial volumes $V_{c a, j}$, $V_{c c, j}$ and $V_{c v, j}$ are computed from the average pressure in each subcompartment. Thus at the beginning of the quasi-diastolic period the latter characteristic quantities obtain an initial value. At $t=0$ an intervention is not necessary. The time constants related to this part of the model are longer than the duration of one heart cycle. Therefore, sometimes several beats are necessary to approximate a stationary situation. 


\section{Literature}

1. Bassingthwaighte, J.B., Yipintsoi, T., Harvey, R.B.: Microvasculature of the dog left ventricular myocardium. Microvasc Res 7, 229 - 249 (1974).

2. Bohlen, H.G., Gore, R.W., Hutchins, P.I1.: Comparison of microvascular pressures in normal and spontaneously hypertensive rats. Ificrovasc Res $\underline{13}, 125-130(1977)$.

3. Durrer, D.: Electrical aspects of human cardiac activity a clinical physiological approach to exitation and stimulation. Cardiovasc Res 2, 1 - 18 (1968).

4. Durrer, D., van Dam, R.Th., Freud, G.E., Janse, M.J., Meyler, F.L., Arzbaecher, R.C.: Total exitation of the isolated human heart. Circulation $\underline{41}, 899-912(1970)$.

5. Grant, R.P.: Notes on muscular architecture of the left ventricie. Circulation 32, $301-307$ (1965).

6. Grayson, J., Davidson, J.W., Fitzgerald - Finch, A., Scott, C.: The functional morphology of the coronary microcirculation in the dog. 11icrovasc Res $8,20-43(1974)$.

7. Hanna, W.T.: A simulation of human heart function. Biophys J 13, $603-621$ (1973).

8. Henquell, L., Lacelle, P.L., Honig, C.R.: Capillary diameter in rat heart in situ: Relation to erythrocyte deformability, $\mathrm{O}_{2}$ transport, and transmural 02 gradients. Microvasc Res 12, 259 - 274 (1976).

9. Hess, D.S., Bache, R.J.: Transmural distribution of myocardial bloodflow during systole in the awake dog. Circ Res 38, 5 - 15 (1976).

10. Hort, $W_{.}:$Makroskopische und mikrometrische Untersuchungen am Myocard verschieden stark gefüllter linker Kammern. Virchows Arch (Pathol Anat) $333,523-564(1960)$.

11. Hugenholtz, P.G., Kaplan, E., Hill, E.: Determination of left ventricular wall thickness by angiocardiography. Am Heart J 78, 513 - 522 (1969).

12. Liedtke, A.J., Pasternae, A., Sonnenblick, E.H., Gorlin, R.: Changes in canine ventricular dimensions with acute changes in preload and afterload. Am J Physiol 223, 820 - 827 (1972).

13. Lower: Tractatus de Corde, London (1669) 26 - 28, fig. 6, 7, 8, tab. 2.

14. Mayrovitz, H.N., Wiedeman, M.P.: Microvascular hemodynamic variations accompanying microvessel dimensional changes. Microvasc Res 10, $322-339$ (1975).

15. Hchale, P.A., Greenfield, J.C.: Evaluation of several geometric models for estimation of left ventricular circumferential wall stress. Circ Res 33 , $303-312(1973)$. 
16. Reneman, R.S., Spencer, M.P.: The use of diastolic reactive hyperemia to evaluate the coronary vascular systell. Ann Trorac Surg 13, $477-487$ (1972).

17. Robb, J.S., Robb, R.C.: The normal heart: anatomy and physiology of the structural units. Am Heart J 23, 455 - 467 (1942).

18. Ross, J. Jr., Sonnenblick, E.H., Cove11, J.H., et al.: The architecture of the heart in systole and diastole: technique of rapid fixation and analysis of left ventricular geometry. Circ Res 21, $409-421$ (1967).

19. Ross, M.A., Streeter, D.D.: Nonuniform subendocardial fiber orientation in the macaque left ventricle. Eur J Card 3, 229 - 247 (1975).

20. Rushmer, R.F., Crystal, D.K., lagner, C.: The functional anatomy of ventricular contraction. Circ Res 1, 162 - 170 (1953).

21. Sandler, H., Gnista, D.N.: Mechanical and dynamic implications of dimensional measurements of the left ventricle. Fed Proc 28, $1344-1350$ (1969).

22. Sandler, H., Alderman, E.: Determination of left ventricular size and shape. Circ Res 34, $1-8(1974)$.

23. Scher, A.M., Young, A.C., Maimigren, A.L., et al.: Spread of electrical activity through the wall of the ventricle. Circ Res 1, $539-547$ (1953).

24. Scher, A.M., Young, A.C.: The pathway of ventricular depolarisation in the dog. Circ Res 4, $461-469$ (1956).

25. Scott Rankin, J., McHale, P., Arentzen, C.E., Ling, D., Greenfield, J.C., Anderson, R.W.: The three-dimensional dynamic geometry of the left ventricle in the conscious dog. Circ Res 39, $304-313$ (1976).

26. Sidney Harris, A.: The spread of excitation in turtle, dog, cat and monkey ventricles. An J Physiol 134, 319 - 332 (1941).

27. Sonnenblick, E.H., Ross, J. Jr., Covel1, J.H., Spotnitz, H.M., Spiro, D.: The ultrastructure of the heart in systole and diastole: changes in sarcomere length. Circ Res 21, 423 - 431 (1967).

28. Spotnitz, H.M., Sonnenblick, E.H., Spiro, D.: Relation of ultrastructure to function in intact heart. Sarcomere structure relative to pressurevolume curves of intact left ventricles of dog and cat. Circ Res 18, 49 66 (1966).

29. Streeter, D.D., Spotnitz, H.M., Patel, D.P., Ross, J., Sonnenblick, E.H.: Fiberorientation in the canine left ventricle during diastole and systole. Circ Res 24, 339 - 347 (1969).

30. Streeter, D.D. Jr., Hanna, W.T.: Engineering mechanics for successive states in canine left ventricular myocardium. II Fiberangle and sarcomere length. Circ Res 33, $656-664$ (1973).

31. Troy, B.L., Pombo, J., Rackley, C.E.: Heasurement of left ventricular wall. Thickness and mass by echocardiography. Circulation 45, $602-611$ (1972). 
32. Westerhof, N., Elzinga, G., van den Bos, G.C.: Influence of central and peripheral changes on the hydraulic input impedance of the systemic arterial tree. Med Biol Eng $710-722$ (1973).

33. Wong, A.Y.K., Rautaharju, P.H.: Relation of sarcomere length to filling pressures in normal and hyperthrophied hearts. Bull Math Biophysics 33, $203-214(1971)$.

34. Yoran, C., Cove11, J.H., Ross, J.: Structural basis for the ascending limb of left ventricular function. Circ Res $32,297-303$ (1973). 
10. RESULTS

\subsection{Introduction}

In this chapter it is shown that the present model of the dynamics of the left ventricle and the coronary circulation is a useful tool to study the mechanics of the heart and its effect on the coronary circulation. To this purpose the cardiac cycle of a large dog is simulated by the model under various hemodynamic conditions. If possible, the results are compared with experimental results, as reported in literature or obtained in our laboratory.

In section 10.2 the generation of a cardiac cycle is described, in which the parameter values as discussed in the previous chapters, are used. The results are briefly discussed and, in view of discrepancies between these results and those obtained in animal experiments, new values of some parameters are proposed (section 10.3). After substitution of these new values in the model another cardiac cycle is simulated. The simulation, thus obtained, is defined as the simulation of a normal cardiac cycle, to which further simulations are referred. In section 10.4 the results of several simulations of deviant cardiac cycles are presented.

\subsection{Simulation of the cardiac cycle with parameter values as discussed in the}

\section{previous chapters}

In this section a cardiac cycle is simulated by the model, using the values of various parameters as discussed in the previous chapters. The first part of this section reviews the values of these parameters, whereupon the results of the simulation are shown, followed by a brief discussion.

The values of the parameters, concerning the geometry of the left ventricle are obtained from section 9.2.1. The end-diastolic dimensions of the simulated left ventricle are:

- inner radius $\left(R_{i v}\right)=22.4 \mathrm{~mm}$

- outer radius $\left(R_{\text {ov }}\right)=34.4 \mathrm{~min}$

- height $\quad\left(H_{v}\right)=76.6 \mathrm{mn}$

and the number of subcylinders is:

$$
n=8
$$


From relation (9.2.7) it follows that for the normal end-diastolic volume of the left ventricle it holds

$$
v_{\text {Iv,ed }}=120.7 \mathrm{mT}
$$

For the velocity of the depolarization wave front across the myocardial wall $\left(v_{\text {dep }}\right)$, as discussed in section 9.2 .4 , it holds

$$
v_{\text {dep }}=0.4 \mathrm{~ms}^{-1}
$$

The course of the fiber orientation and the sarcomere length across the left ventricular wall are discussed in section 9.2 .2 and 9.2 .3 , respectively. The end-diastolic fiber angles $\left(B_{0, j}\right)$ based on data obtained by streeter et al. ${ }^{8)}$, applied to 8 subcylinders, are presented in table 10.1. In the same table the end-diastolic values of the sarcomere length in each subcylinder, obtained by interpolation of the results of Yoran et al. ${ }^{9}$ ) are presented.

Table 10.1. End-diastolic fiber angle $\left(B_{0, j}\right)$ and sarcomere length $\left(l_{s o, j}\right)$ in

\begin{tabular}{|c|c|c|c|c|c|c|c|c|}
\hline \multirow[b]{2}{*}{ j } & \multicolumn{2}{|c|}{ subendocardium } & & & & & \multicolumn{2}{|c|}{ subepicardium } \\
\hline & 1 & 2 & 3 & 4 & 5 & 6 & 7 & 8 \\
\hline $0, j \quad[\mathrm{rad}]$ & 0.733 & 0.419 & 0.175 & 0.000 & -0.122 & -0.262 & -0.436 & -0.855 \\
\hline so,j [ & 1.95 & 2.01 & 2.06 & 2.04 & 2.03 & 2.01 & 2.00 & 1.98 \\
\hline
\end{tabular}
each subcylinder $j$.

The contractile behavior of the myocardial material is discussed in section 5.3. The values of the parameters which are related to the passive element are:

- sarcomere length under zero load

(a) $=1.95 \quad \mathrm{~mm}$

- parameter governing exponential increase of the force with the sarcomere length $\quad(b)=8.0 \quad \mathrm{um}^{-1}$

- proportionality factor passive force $\left(G_{p o}\right)=4.4 \cdot 10^{8} \mathrm{~N} \mathrm{~m}^{-3}$

The values of the parameters, which are related to the description of the active element by Hill's equation, are:

- proportionality factor active force

$$
\begin{aligned}
& \left(G_{\mathrm{ao}}\right)=2.8 \cdot 10^{10} \mathrm{~N} \mathrm{~m}^{-3} \\
& \left(\mathrm{v}_{\mathrm{ao}}\right)=5.8 \quad \mathrm{Im} \mathrm{s} \mathrm{m}^{-1}
\end{aligned}
$$$$
\text { - proportionality factor velocity of }
$$
shortening 
- maximum value of the degree of activation

$$
\left(A_{\max }\right)=2.0
$$

The parameters, which govern the behavior of the activation as a function of time and sarcometer length, are:

- rise time of activation

$$
\begin{aligned}
& \left(\tau_{s 1}\right)=0.04 \mathrm{~s} \\
& \left(\tau_{s 2}\right)=0.04 \mathrm{~s}
\end{aligned}
$$

- decay time of activation

- parameters which relate the moment of

decay of activation to:

$\begin{aligned} \text { sarcomer length: constant } & \left(1_{s 1}\right) & =1 \mathrm{~km} \\ \text { slope } & \left(v_{s l}\right) & =2.2 \mathrm{~m} \mathrm{~s}^{-1} \\ \text { moment of sarcomere shortening } & \left(b_{1}\right) & =5 \mathrm{~mm}^{-1}\end{aligned}$

Most of these values are presented by the group of relations $(5.2 .21)$. The value of $A_{\text {max }}$, however, is chosen to be lower than presumed in relation (5.3.21) because this value depends on temperature (section 5.3). The present value of $A_{\text {max }}$ is based on a temperature of $37^{\circ} \mathrm{C}$. Related to this change in value of $A_{\max }$ new values of $v_{a 0}$ and $G_{a 0}$ are given.

In the simulation it is assumed that:

- the end-diastolic aortic pressure $\left(\mathrm{p}_{\text {ao,ed }}\right)=10000 \mathrm{~N} \mathrm{~m}^{-2}$

- the right atrial pressure $\left(p_{r a}\right)=800 \mathrm{~N} \mathrm{~m}^{-2}$

The aortic input impedance is discussed in section 9.2.5. The proposed values of the paraneters are:

- peripheral resistance

$$
\begin{aligned}
& \left(R_{p}\right)=1.2 \cdot 10^{8} \mathrm{~N} \mathrm{~s} \mathrm{~m}^{-5} \\
& \left(Z_{\text {ao }}\right)=1.7 \cdot 10^{7} \mathrm{~N} \mathrm{~s} \mathrm{~m}^{-5} \\
& \left(C_{\text {art }}\right)=3.0 \cdot 10^{-8} \mathrm{~N}^{-1} \mathrm{~m}^{-5}
\end{aligned}
$$

- arterial capacitance

In relation to this it should be remarked, that these parameter values belong to a large dog.

For the large coronary arteries (I.D. > $400 \mu \mathrm{m}$ ) the parameter values, mentioned in relation (9.2.14), are used. These values, which are defined at an internal pressure of $13300 \mathrm{~N} \mathrm{~m}^{-2}(=100 \mathrm{mmHg})$, are:

- volume

- capacitance

$$
\begin{aligned}
& v_{c e}=1.33 \quad \mathrm{ml} \\
& c_{c e}=1.4 \cdot 10^{-11} \mathrm{~N}^{-1} \mathrm{~m}^{5}
\end{aligned}
$$


- resistance

- inertance

$$
\begin{aligned}
& R_{\text {ce }}=5.7 \cdot 10^{8} \mathrm{~N} \mathrm{~s} \mathrm{~m}^{-5} \\
& L_{\text {ce }}=7.6 \cdot 10^{-6} \mathrm{~N} \mathrm{~s}^{2} \mathrm{~m}^{-5}
\end{aligned}
$$

The values of the parameters of the coronary microcirculation are discussed in section 9.2.8. For the sake of convenience, the parameters which describe the properties of the microcirculatory compartment are normalized per unit of muscle volume. In this way the values of the parameters in the equations $(6.3 .2)$ and $(6.3 .3)$ are:

in the arteriolar subcompartment (a)

$$
\begin{aligned}
& S_{p a}=3.5 \cdot 10^{14} \mathrm{~N} \mathrm{~m}^{-2} \\
& S_{r a}=357 \\
& S_{C a}=7
\end{aligned}
$$

in the capillary subcompartment (c)

$$
\begin{aligned}
& S_{p c}=6.65 \cdot 10^{12} \mathrm{~N} \mathrm{~m}^{-2} \\
& S_{r c}=317 \\
& s_{c c}=7
\end{aligned}
$$

and in the venular subcompartment ( $v$ )

$$
\begin{array}{ll}
s_{p v}=1.79 \cdot 10^{12} \mathrm{~N} \mathrm{~m}^{-2} \\
s_{r v}=325 & \mathrm{~N} \mathrm{~s} \mathrm{~m}^{-2}(10.2 .6 \mathrm{C}) \\
s_{C V} & =7
\end{array}
$$

where $S_{p}=$ proportionality factor in the relation between transmural pressure and volume of the subcompartment

$S_{r}=$ proportionality factor in the relation between resistance and volume of the subcompartment

$s_{C}=$ exponent in the relation between transmural pressure and volume of the subcompartment

The parameters, mentioned in the group of relations (10.2.6) are supposed to be the same in all subcylinders, an assumption which is probably not true, as indicated by experiments of Myers et al. 3) (section 10.3).

In the present simulation it is assumed that there is no vaso-activity, i.e. the vasoconstrictive pressure is assumed to be equal to zero. In this way complete coronary vasodilation is simulated.

The duration of the cardiac cycle is chosen to be: 
$t_{\text {cycl }}=600 \mathrm{~ms}$

A fourth-oraer Runge Kutta method is used to solve the system of differential equations (4.1.1). The time increments applied are fixed at 4 ms. The latter value is chosen on the basis of the smallest time constant in the system ( $=$ $10 \mathrm{~ms}$ ) which is related to the inertance and capacitance in the large coronary arteries (I.D. $\because 400 \quad$ H. I I). Another rapid phenomenon in the model is the rise of the aortic flow after opening of the aortic valve.

The results of the simulation of a cardiac cycle, obtained with the parameter values mentioned, are shown in fig. 10.1 .
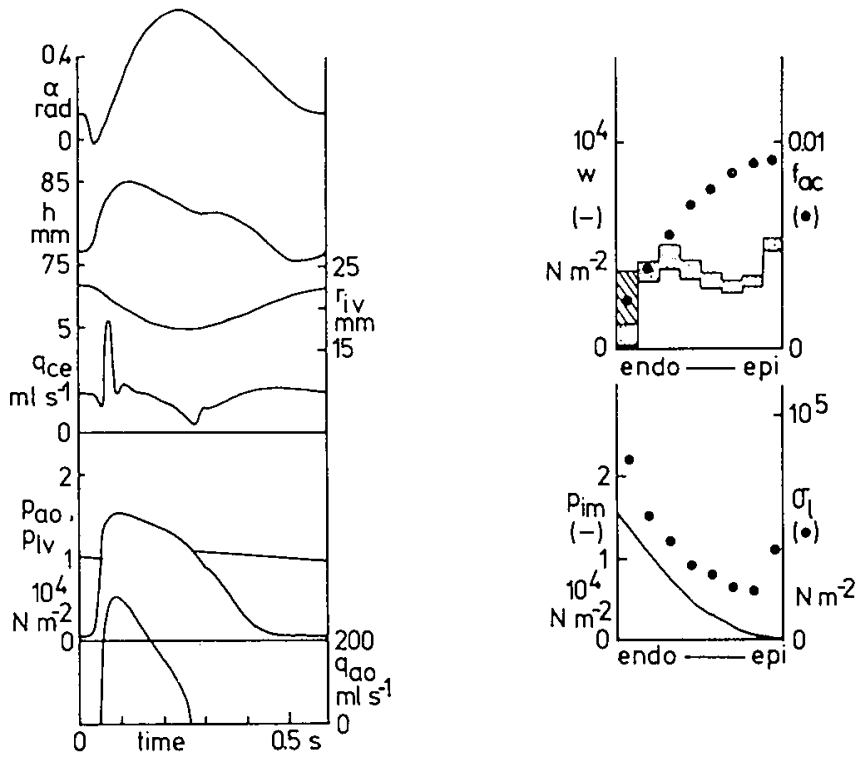

Figure 10.1 .

Results of a simulation of a normal cardiac cycle, using the values of parameters, as discussed in the previous chapters.

$p_{\text {ao }}=$ aortic pressure

$\mathrm{p}_{\mathrm{lv}}^{\mathrm{aO}}=\mathrm{left}$ ventricular pressure

$q_{a 0}=$ aortic volume flow

$q_{c e}=$ coronary artery volume flow

${ }_{h} \mathrm{ce}=$ height of the cylinder

$r_{i v}=$ inner radius of the cylinder

$\alpha^{i v}=$ torsion angle of the cylinder

$\mathrm{p}_{\mathrm{im}}=$ intramyocardial pressure at $t=120 \mathrm{~ms}$

$\sigma_{1}=$ tensile muscle stress $t=120 \mathrm{~ms}$

$W=$ generated mechanical specific energy per beat

$f_{a c}=$ coronary perfusion flow per unit of muscle volume, integrated over one beat

In relation to $w$ the shaded area denotes the loss of energy due to stretching of activated muscle during ejection, and dotted area denotes the loss of energy due to stretching of activated muscle during the left ventricular filling phase. 
The simulated cardiac cycle looks normal in several respects, such as:

- the time course of the aortic volume flow

- the time course of the aortic pressure

- an ejection fraction of 36

- an increase of the base to apex distance during the isovolumic contraction

- a decrease of the base to apex distance as well as of the inner radius of the left ventricle during the ejection phase.

However, the simulation also shows several discrepancies with the physiological situation, such as:

- the rise of left ventricular pressure is too fast

- the decay of left ventricular pressure is too slow

- the duration of systole is long as compared with the duration of the cardiac cycle, which results in an unphysiological mitral inflow during the relaxation phase

- the increase of the base to apex distance during the isovolumic contraction is too large

- the systolic component of coronary artery volume flow is not lower than its diastolic component

- the perfusion of the subendocardial layers is much lower than the perfusion of the subepicardial layers.

Moreover, in the simulation the distribution of the generated mechanical specific energy across the myocardial wall is not homogenous. Although this quantity is never measured in animal experiments, it seems reasonable to assume that the same amount of muscle would generate the same amount of power.

In the following the characteristics of the simulation, presented in fig. 10.1, are discussed in more detail. In the simulation the maximum rate of left ventricular pressure increase during the isovolumic phase amounts to $7 \cdot 10^{5} \mathrm{~N} \mathrm{~m}^{-2} \mathrm{~s}^{-1}$, which value is approximately twice the normal value. The decay of the left ventricular pressure maximally anounts to $1.6 \cdot 10^{5} \mathrm{~N} \mathrm{~m}^{-2}$ $s^{-1}$, which is approximately half the normal value. Moreover, the duration of the simulated ejection period is $36 \%$ of the duration of the cardiac cycle. However, at a duration of the cardiac cycle of $600 \mathrm{~ms}$ a nomal value of this percentage is 30 or less (own observation, fig. 10.3). The latter three discrepancies can be met by an adaptation of the time course of the degree of activation.

It is not surprising that discrepancies in this time course are present 
because calculation of the related parameters was mainly based on the results obtained in $\cdots$ experiments on papillary muscle of different animals and at different operating temperatures (chapter 5). Horeover, in most of these experiments the muscle length in stead of the sarcomere length was controlled, which situation is associated with large artifacts ${ }^{4)}$ due to unequal contraction along the muscle under investigation. To our knowledge experimental data on the in witro contractile behavior of canine cardiac muscle are not available. In sive experiments the best method to study this contractile behavior is using a model of cardiac mechanics to relate wall stress to more accurately measurable quantities such as left ventricular pressure and dimensions. When using the present model, this strategy means that the parameters which govern the contractile behavior of cardiac muscle are varied until left ventricular pressure and dimensions during the cardiac cycle, as generated by the model, agree with the experimental situation. In the introduction of this study (chapter 1) this method is denoted as the less preferable method of parameter estimation, but in this case the more preferable method of direct determination of parameter values can not be applied since necessary information is lacking.

Another discrepancy between the present simulation and the animal experiment is the presence of mitral inflow during the simulated left ventricular relaxation phase. Under physiological circumstances this inflow is impossible since in this phase of the cardiac cycle atrial pressure cannot exceed left ventricular pressure and open the mitral valve. In the silulation, however, mitral inflow occurs since this inflow is characterized by a volume flow source. Simulation of mitral inflow by a pressure source would be more realistic, but requires a modification of the model, which is not carried out. The present inaccuracy in the description of mitral flow results in a considerable consumption of mechanical energy by the left ventricle during the ventricular relaxation phase when mitral inflow is present. The distribution of this loss in specific energy across the nyocardial wall is indicated in fig. 10.1 by the dotted area. The discrepancy can be met by shortening the duration of systole, so that mitral inflow occurs after relaxation of the left ventricle.

In the simulation, transmural differences in generated mechanical specific energy are low in the outer 7 subcylinders at the end of the ejection phase (fig. 10.1, blank area). The extremely low value of this mechanical specific energy in the inner subcylinder is due to stretching of the activated subendocardial layer during the first part of systole. Then the fibers in this layer 
are not able to withstand the required force. This process consumes, in stead of produces, mechanical energy. The specific energy, which is lost in this way, is indicated in fig. 10.1 as the shaded area. As shown in the same figure, the fiber stress during systole is high in the subendocardial layers and low in the subepicardial layers. This uneven distribution of stress across the myocardial wall causes unequal shortening of the fibers and, in some cases, even stretching of activated muscle. A more optimal design requires a more uniform distribution of this fiber stress.

In the present simulation the time course of the dimensions of the heart during the cardiac cycle shows some differences with the experimental findings reported by Horwitz et al. ${ }^{2)}$, Sasayama et al. ${ }^{5)}$, and Scott-Rankin et al. 6). They found that during the isovolumic contraction phase the distance of the base to the apex slightly increases, while the inner radius of the left ventricle slightly decreases. During the ejection phase both distances decrease. During the relaxation phase these distances change rather quickly at constant volume of the left ventricular cavity. During diastole the left ventricle is filled, and both the inner radius and, to a minor extent, the base to apex distance increase. Comparable changes are found in the simulation. Quantitatively, however, the increase in height of the cylinder, which is the equivalent of the base to apex distance, is exaggerated in the simulated isovolumic contraction phase. Mechanically, this increase can be understood from an imbalance of axial forces in the cylinder when the fiber orientation is as assumed. This imbalance is met by an increased height of the cylinder which forces the fibers to rotate towards a more axial direction, thus restoring the axial equilibrium of forces. During diastole, the weak forces in the endocardium and other structures in the left ventricular cavity are sufficiently large to keep the cylinder short. During systole, however, forces in the myocardium increase and their influence on the geometry dominates the influence of the passive elastic endocardium and other structures in the left ventricular cavity such as papillary muscles and chordae tendinae. This results in a lengthening of the cylinder. The present explanation is supported by the finding that the geometry during the relaxation phase changes rather rapidly. Then the rather weak endocardial forces regain their influence on the geometry. Changing of initial fiber orientation in a more axial direction results in a smaller increase of the base to apex distance during the isovolumic contraction phase.

Fig. 10.1 also shows the time course of the torsion angle. The torsion an- 
gle decreases during the isometric contraction reaching a minimum and increases gradually during the ejection phase. During the relaxation phase this angle decreases to the diastolic level. During diastole the torsion angle is approximately constant. To our knowledge this quantity has never been measured experimentaliy and, therefore, it is discussed in chapter 11, rather than in the present section.

In the simulation the systolic component of coronary artery volume flow is of the same order of magnitude as its diastolic component. Moreover, the perfusion of the subendocardial layers is only $25 \%$ of that in the subepicardial layers. In the biological situation, however, the systolic component of coronary artery volume flow is significantly smaller than the diastolic component, and transmural differences in coronary perfusion are smal1 ${ }^{1}$ ). When studying the coronary volume flow as generated by the model, one must realize that a state of complete coronary vasodilation is simulated. Experimentally this situation is, for instance, present during coronary reactive hyperemia which is defined as the increase in coronary blood flow over the control value following momentary occlusion of a coronary artery.

In the present simulation the low subendocardial perfusion is explained by the neglect of transmural differences in coronary microcirculatory properties. Mean intranyocardial pressure is larger in the subendocardial layers than in the subepicardial layers. Therefore, as compared to the subepicardial layers, in the subendocardial layers the microcirculatory volume is lower and hence, the microcirculatory resistance is higher. This results in a relatively lower mean subendocardial perfusion.

The absence of a pronounced difference between the systolic and diastolic components of coronary artery volume flow in the model is related to this nonuniform transmural distribution of coronary perfusion. In the subendocardial layers systolic perfusion is significantly lower than diastolic perfusion, which is due to a high systolic intramyocardial pressure in the subendocardium. In the subepicardial layers, however, systolic perfusion is larger than diastolic perfusion, because the perfusion pressure during systole is larger than during diastole. In the model subepicardial perfusion is relatively more pronounced than in the normal physiological situation, hence the simulated coronary artery volume flow is determined to a larger extent by subepicardial perfusion. Therefore, it can be expected that during systole a normal, physiological suppression of coronary artery volume flow is less pronounced in the present simulation. By introduction of transmural gradients in microcirculato- 
ry properties as are found by llyers et al. ${ }^{3)}$ the influence of the subendocardial perfusion on total coronary artery volume flow is more pronounced, resulting in larger differences between systolic and diastolic coronary volume flow. The situation, thus obtained, is probably more realistic.

\subsection{Adaptation of several parameters in order to obtain a more realistic simu-} lation of the cardial cycle

In the previous section a simulation of the cardiac cycle was discussed. In view of the differences between the simulation and the normal situation in the animal, as discussed in section 10.2, new values of several parameters are estimated in the present section. Discrepancies between the simulation and the physiological situation are found with respect to the time course of the left ventricular pressure. To obtain a more normal value of the rate of increase of ventricular pressure, the rise time of left ventricular pressure is lowered by changing in relation (5.3.18) the exponent 4 to 3 . In addition, for the parameter $\left(\tau_{s 1}\right)$ which governs the rise time of the degree of activation, a larger value is chosen:

$$
\tau_{\mathrm{s} 1}=50 \mathrm{~ms}
$$

To obtain a more realistic decay of the left ventricular pressure the decay time of left ventricular pressure during relaxation is lowered by choosing a lower value of the parameter $\left(\tau_{\mathrm{s} 2}\right)$ which governs this decay time:

$$
\tau_{\mathrm{s} 2}=30 \mathrm{~ms}
$$

The duration of systole had to be shortened, which is carried out by changing the related parameters

$$
\begin{aligned}
& v_{s 1}=0.40 \mu \mathrm{m} \mathrm{s}^{-1} \\
& b_{1}=1.5 \mathrm{~mm}^{-1}
\end{aligned}
$$

The non-uniform distribution across the wall of fiber stress, as calculated in the simulation, is met by changing the initial fiber orientation in each subcylinder. In the same procedure the exaggeration of the increase in height in early-systole is met by initially directing the muscle fibers more axially. Transmural differences in generated mechanical specific energy are eliminated 
by choosing an appropriate initial sarconere length in each subsylinder. Table 10.2 shows the new values of the initial fiber angle and sarcomere length in each subcylinder.

Table 10.2. New, adapted, values of the end-diastolic fiber angle $\left(\beta_{0, j}\right)$, sarcomere length $\left(l_{s o, j}\right)$ and microcirculatory parameters $\left(S_{p a}, S_{p c, j}\right.$ and $S_{p v, j}$ in each subcylinder $j$.

\begin{tabular}{|c|c|c|c|c|c|}
\hline j & $\begin{array}{l}\hat{B}_{0, j} \\
\mathrm{rad}\end{array}$ & $\begin{array}{l}\text { ' } \\
\text { so }, \mathrm{j} \\
\text { jum }\end{array}$ & $\begin{array}{l}S_{p a, j} \\
N^{-2}\end{array}$ & $\begin{array}{l}S_{p c, j} \\
\mathrm{~N} \mathrm{~m}^{-2}\end{array}$ & $\begin{array}{l}S_{p v, j} \\
N m^{-2}\end{array}$ \\
\hline & 0.80 & 2.05 & $1.18 \cdot 10^{13}$ & $2.25 \cdot 10^{10}$ & $6.06 \cdot 10^{10}$ \\
\hline & 0.50 & 2.08 & $1.85 \cdot 10^{13}$ & $3.52 \cdot 10^{11}$ & $9.50 \cdot 10^{10}$ \\
\hline & 0.30 & 2.10 & $3.78 \cdot 10^{13}$ & $7.19 \cdot 10^{11}$ & $1.94 \cdot 10^{11}$ \\
\hline & 0.10 & 2.10 & $7.56 \cdot 10^{13}$ & $1.44 \cdot 10^{12}$ & $3.86 \cdot 10^{11}$ \\
\hline & -0.05 & 2.09 & $1.35 \cdot 10^{14}$ & $2.57 \cdot 10^{12}$ & $6.93 \cdot 10^{11}$ \\
\hline & -0.10 & 2.09 & $2.26 \cdot 10^{14}$ & $3.69 \cdot 10^{12}$ & $1.16 \cdot 10^{12}$ \\
\hline & -0.70 & 2.08 & $3.28 \cdot 10^{14}$ & $6.24 \cdot 10^{12}$ & $1.68 \cdot 10^{12}$ \\
\hline & -1.40 & 2.04 & $3.50 \cdot 10^{14}$ & $6.65 \cdot 10^{12}$ & $1.79 \cdot 10^{12}$ \\
\hline
\end{tabular}

Since in the model coronary perfusion was found to be not uniformly distributed across the wall, transmural gradients of the microcirculatory parameters are introduced. The endocardial flow resistance is lowered by an increase in coronary volume, which is attained by lowering te value of the parameter $S_{p}$ in each subcompartment. Within one subcylinder the parameter $S_{p}$, belonging to the arteriolar, capillary and venular subcompartment, respectively, are multiplied by the same correction factor, so that the change in coronary vascular resistance is uniformly distributed over all subcompartments. The new values of $S_{p a, j}, S_{p c, j}$ and $S_{p v, j}$ are also given in Table 10.2. The simulation as obtained with the new parameter values is represented in fig. 10.2. These results differ from the first simulation, presented in fig. 10.1 , in the following aspects:

- slower increase of the left ventricular pressure during the isovolumic contraction phase

- faster decrease of the left ventricular pressure during the isovolumic relaxation phase

- shorter duration of systole 

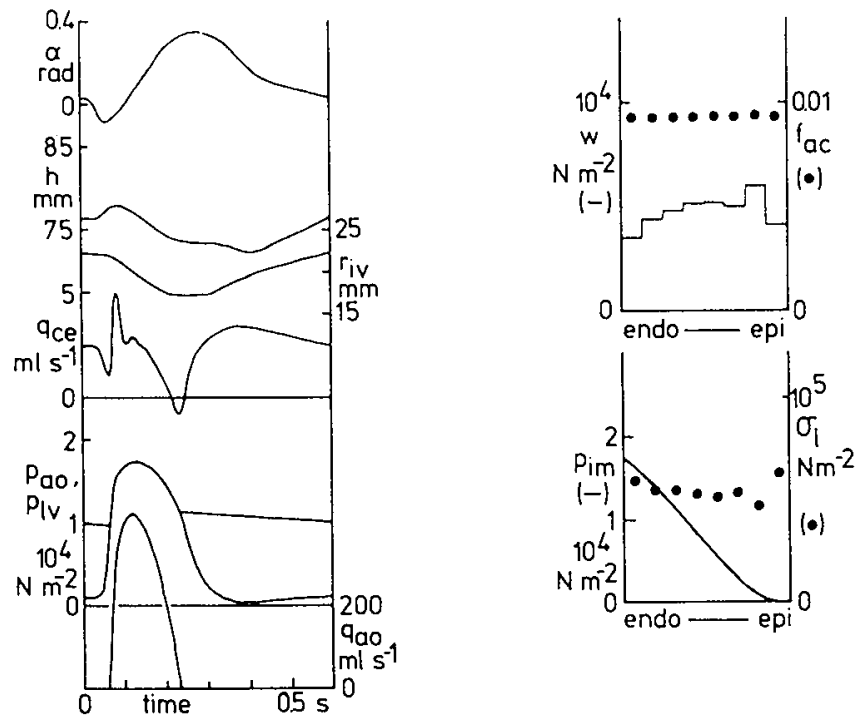

Figure 10.2.

Results of a simulation of a normal cardiac cycle after adaptation of some parameters. This simulation is used as the simulation of a normal cardiac cycle, to which deviant cardiac cycles are referred.

Figure 10.3.

Experimental results of a normal cardiac cycle $(\operatorname{dog} 25 \mathrm{~kg})$.

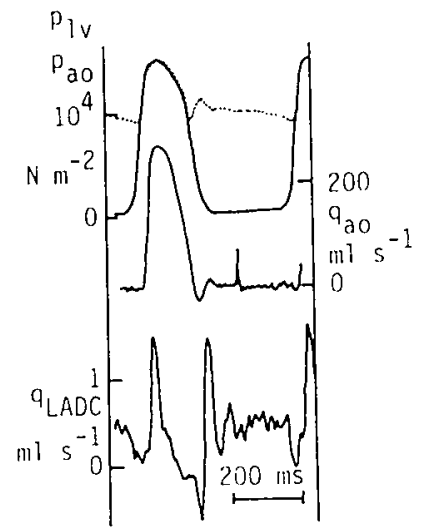


- smaller increase of the height of the cylinder during the isovolumic contraction phase

- More uniforil distribution of the generated mechanical specific energy across the myocardial wall

- more uniform distribution of the systolic tensile muscle stress across the myocardial wall, resulting in a different transmural course of the intramyocardial pressure

- no significant stretching of activated muscle

- uniform distribution of the coronary perfusion across the wall

- larger volume of the subendocardial coronary vasculature

- more realistic time course of the coronary artery volume flow, i.e. a relatively larger diastolic component.

Experimentally, a similar cardiac cycle is obtained from a dog weighing $25 \mathrm{~kg}$ (fig. 10.3). The simulation agrees fairly well with the experiments in the following aspects:

- time course of left ventricular and aortic pressures

- time course of aortic volume flow

- time course of the coronary arterial volume flow as far as minimum flow before opening of the aortic valve, peak flow after opening of the aortic valve, decline in flow during systole, backflow during end-systole and high forward flow during diastole are concerned.

A discrepancy is the absence of the peak in coronary artery volume flow in the simulation, directly after closure of the aortic valve. Moreover, in the simulation the relaxation phase of the myocardium seems to be prolonged, as is shown by the rather slow decrease of left ventricular pressure as well as by the rather slow increase of coronary artery flow during this phase of the cardiac cycle.

The simulation shows, that the systolic transmural gradient of intramyocardial pressure is approximately constant in the subendocardial and center layers, and is low in the subepicardial layers. This implies that from the endocardium the intramyocardial pressure is decreasing approximately 1 inearly until a plateau of low values in the subepicardial layers is reached. At the epicardium the intramyocardial pressure is zero.

\subsection{Simulation of deviant cardiac cycles}

The present section deals with simulations of deviant cardiac cycles. To compare the values of the parameters in the deviant situations with normal, 
the normal situation must be defined. The definition of the normal situation shows some arbitrariness. Several of the presented values of the parameters are based upon the experiment on a dog from which some data are shown in fig. 10.3. Other values of parameters are obtained from in vitro and in vivo studies which are discussed in the previous chapters. Normal values of several relevant parameters thus obtained are supposed to be:

- duration of the cardiac cycle

- end-diastolic left ventricular volume

- peripheral resistance

- aortic characteristic impedance

- velocity of the depolarization wave front

- resistance of the large coronary arteries

- inertance of the large coronary arteries

$t_{c y c l}$
$v_{1 v, e d}$
$R_{p}$
$z_{a o}$
$v_{\text {dep }}$
$R_{c e}$
$L_{c e}$

$=600 \quad \mathrm{~ms}$

$=120 \mathrm{ml}$

$=1.2 \cdot 10^{8} \mathrm{Ns} \mathrm{m}^{-5}$

$=1.7 \cdot 10^{-7} \mathrm{~N} \mathrm{~s} \mathrm{~m}^{-5}$

$=0.4$

$\mathrm{ms}^{-1}$

$=5.7 \cdot 10^{8} \mathrm{~N} \mathrm{~s} \mathrm{~m} \mathrm{~m}^{-5}$

The following deviations in the cardiac cycle are simulated:

1. an increased end-diastolic volume in combination with a decreased heart rate

$v_{17, e d}=170$

$7.6 \cdot 10^{6} \quad \mathrm{Ns}^{2} \mathrm{~m}^{-5}$

$\begin{array}{lll}v_{\text {lv,ed }}=170 & \mathrm{ml} \\ t_{\text {cycl }}=780 & \mathrm{~ms}\end{array}$

2. a decreased end-diastolic volume in combination with an increased heart rate

$\begin{array}{lll}v_{1 v, e d} & =70 & \mathrm{ml} \\ t_{\text {cycl }} & =420 & \mathrm{~ms}\end{array}$

3. an isovolumic contraction

$\begin{array}{ll}R_{p} & =1.2 \cdot 10^{18} \mathrm{~N} \mathrm{~s} \mathrm{~m}^{-5} \\ Z_{a 0} & =1.7 \cdot 10^{11} \mathrm{~N} \mathrm{~s} \mathrm{~m}^{-5}\end{array}$

4. an increased peripheral resistance $R_{p}$

$=3.5 \cdot 10^{8} \quad \mathrm{~N} \mathrm{~s} \mathrm{~m} \mathrm{~m}^{-5}$

5. a coronary artery stenosis

6. a disturbed spread of activation

$\begin{array}{ll}R_{c e} & =10^{10} \mathrm{~N} \mathrm{~s} \mathrm{~m}^{-5} \\ L_{c e} & =3 \cdot 10^{7} \mathrm{~N} \mathrm{~s}^{2} \mathrm{~m}^{-5}\end{array}$

$v_{\text {dep }}=-0.4 \quad \mathrm{~m} \mathrm{~s}^{-1}$

Ad 1: Fig. 10.4 shows a simulation of a cardiac cycle which is characterized by an increased end-diastolic volume and a decreased heart rate. Experimentally, a similar situation is created by infusing intravenously $200 \mathrm{ml}$ of saline into a dog weighing $25 \mathrm{~kg}$ (fig. 10.5). The simulation agrees with the animal experiment in the following aspects, as compared to normal:

- higher maximum ventricular and aortic pressures

- higher stroke volume, which is defined as the volume of blood, which is ejected into the aorta during systole 

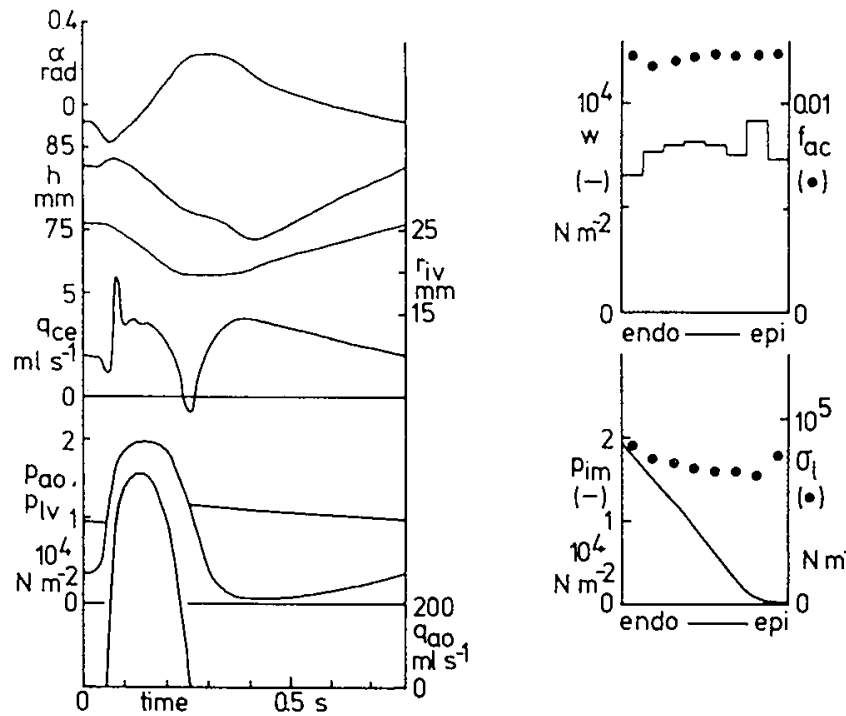

Figure 10,4.

Results of a simulation of a cardiac cycle, which is characterized by a larger end-diastolic left ventricular volume and a lower heart rate, as compared to normal (fig. 10.2, symbols: see text fig. 10.1).

Figure 10.5

Experimental results of a cardiac cycle, which is characterized by a larger enddiastolic left ventricular volume and a lower heart rate as compared to norma] (fig. 10.3; $\operatorname{dog} 25 \mathrm{~kg}$ ).

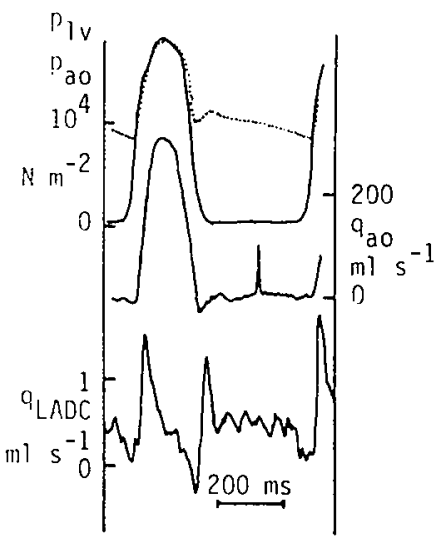


- longer duration of the ejection phase

- longer interval between the start of ejection and the moment of reaching the maximum left ventricular pressure

- higher coronary artery volume flow during systole.

From the simulation it appears, as compared to nomal, that:

- the transmural gradients of tensile muscle stress, mechanical specific energy and coronary perfusion are inconsiderable as in the simulation of a normal cardiac cycle

- the ejection fraction which is defined as the ratio of stroke volume and end-diastolic left ventricular volume, is slightly higher (46., normal 42 (i)

- the increase of the base to apex distance during the isovolumic contraction phase is less pronounced

- the ratio of coronary perfusion and generated mechanical specific energy is less in all parts of the ventricular wall.

Ad 2: Fig. 10.6 shows the simulation of a cardiac cycle which is characterized by a decreased end-diastolic left ventricular volume and a high heart rate. Experimentally, a similar situation is created by arterial bleeding of $300 \mathrm{ml}$ in the same dog as mentioned in the previous experiment (fig. 10.7). The simulation agrees with the animal experiment in the following aspects, as compared to normal:

- lower maximum systolic aortic and left ventricular pressure

- lower end-diastolic aortic and left ventricular pressure

- Jower stroke volume

- shorter duration of the ejection phase

- shorter interval between the start of ejection and the moment of reaching the maximum left ventricular pressure

- lower coronary arterial volume flow during systole.

From the simulation it appears, as compared to normal, that:

- as compared to their mean values, transmural differences in tensile muscle stress, mechanical specific energy and coronary perfusion are relatively more pronounced, since the average values of these quantities across the wall are relatively less

- the ejection fraction is less than normal ( $37 \%$, normal $42 \%$ )

- the increase of the base to apex distance during the isovolubic contraction phase is more pronounced, other changes in this distance are less pronounced; the duration of the systole is long as compared with the duration of the cardiac cycle, which is shown by the absence of a 

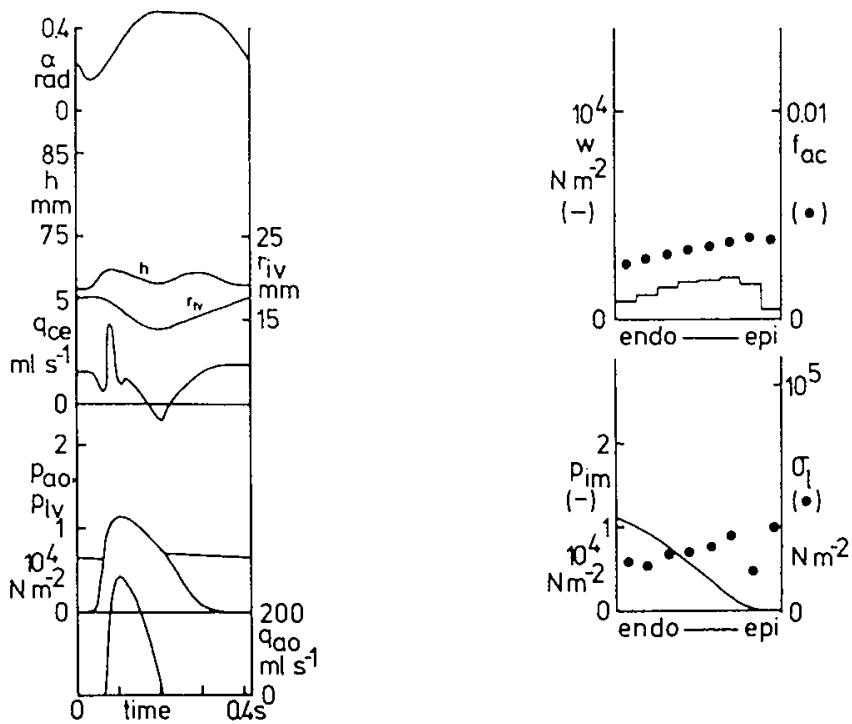

Figure 10.6 .

Results of a simulation of a cardiac cycle, which is characterized by a smaller end-diastolic left ventricular volume and a higher heart rate, as compared to normal (fig. 10.2, symbols: see text fig. 10.1).

Figure 10.7.

Experimental results of a cardiac cycle, which is characterized by a smaller enddiastolic left ventricular volume and a higher heart rate, as compared to normal

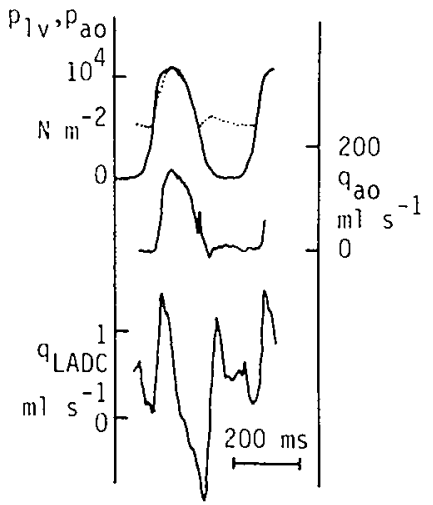
(fig. 10.3; dog $25 \mathrm{~kg}$ ). 
moderate rising value of the base to apex distance during end-diastole

- the ratio of coronary perfusion and developed mechanical specific energy is not affected significantly

- a slightly lower perfusion of the subendocardial than of the subepicardial layers occurs.

A discrepancy with the animal experiment is the rather slow relaxation of the left ventricle in the simulation. In the animal experiment coronary artery volume flow is increased, which is probably due to coronary vasodilation, induced by increased metabolic needs probably due to the increase in heart rate.

Ad 3: Fig. 10.8 shows the simulation of a cardiac cycle which is characterized by a constant left ventricular volume. Experimentally, a similar situation is created by aortic occlusion, introduced during the preceding diastole (fig. 10.9). The simulation agrees with the animal experiment in the following aspects, as compared to normal:

- the maximum systolic left ventricular and aortic pressures are increased (simulation $+70 \%$, experiment $+56 \%$ )

- the duration of systole is increased

- coronary artery volume flow is increased during systole.

The simulation is comparable with the experiment (fig. 10.9) until closure of the aortic valve. The moments of closure of the aortic valve in the animal experiment and the simulation do not coincide because of differences in the aortic input impedance. In the experimental situation the aortic pressure increases during systole, resulting in an increase of the volume of the aorta proximal to the occlusion. This volume increase is due to the capacitive properties of this part of the aorta. During the end of systole the left ventricle relaxates which results in aortic backflow and closure of the aortic valve. The higher aortic pressure is maintained by the capacitance of the part of the aorta proximal to the occlusion. In the simulation this maintenance of the aortic pressure is not present, since the capacitance of the aorta proximal to the occlusion is neglected.

The shaded areas in fig. 10.8 indicate the amount of generated mechanical specific energy which is lost by stretching of activated muscle. From the simulation of an isovolumic cardiac cycle it appears, as compared to normal, that:

- the increase of the base to apex distance during contraction is more 

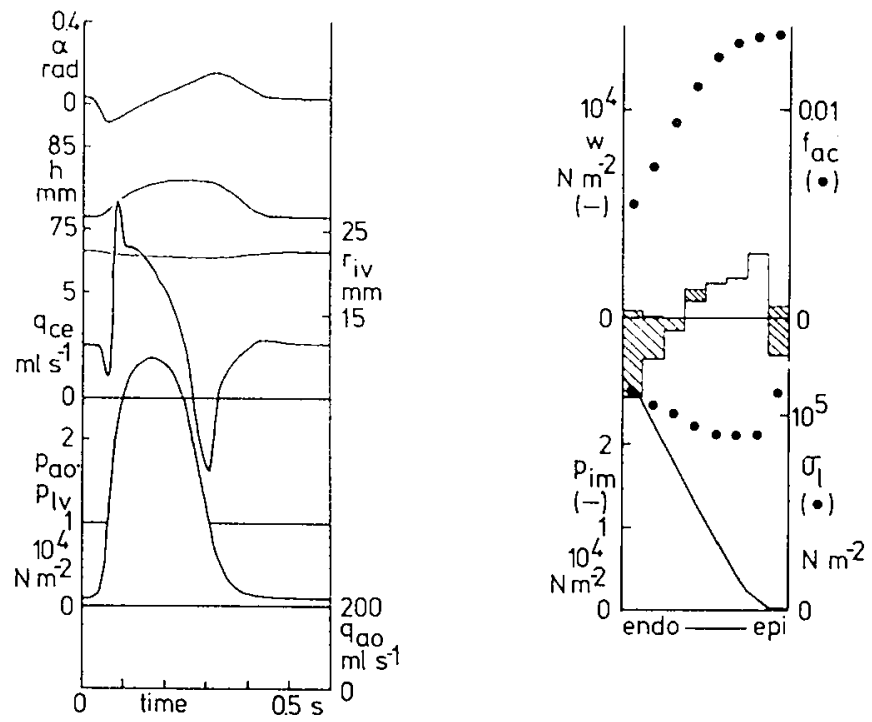

Figure 10.8 .

Results of a simulation of a cardiac cycle, after introduction of an aortic occlusion during the previous diastolic period (symbols: see text fig. 10.1).

Figure 10.9 .

Experimental results of a cardiac cycle after introduction of an aortic occlusion during the previous diastolic period $(\operatorname{dog} 25 \mathrm{~kg})$.

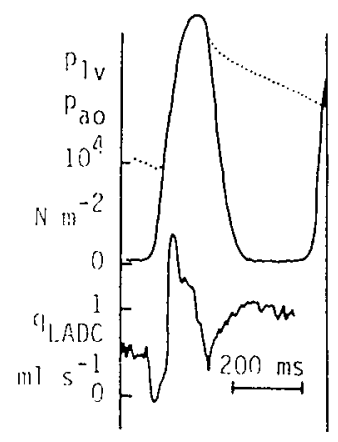


pronounced and prolonged, the changes in torsion angle are of a normal character but of lower amplitude

- subendocardial capillary inflow is low and subepicardial capillary inflow is high.

Ad 4: Fig. 10.10 shows the simulation of a cardiac cycle, which is characterized by a high peripheral resistance. This situation is comparable with hypertension without increased contractility of the myocardium. Some characteristic differences with the simulated normal cardiac cycle are:

- a higher diastolic aortic pressure

- a lower ejection fraction (20\%, normal 42\%)

- a more flattened course of systolic left ventricular pressure

- a more pronounced increase in base to apex distance during the isovolumic contraction phase

- a lower amplitude of changes in the torsion angle

- a relatively higher diastolic component of coronary artery volume flow

- a more irregular distribution of mechanical specific energy across the wall, the shaded areas in fig. 10.10 indicate the amount of generated mechanical specific energy which is lost by stretching of activated muscle

- an increase of the ratio of coronary perfusion and generated mechanical specific energy, especially in the subendocardial layers.

With respect to the latter statement it should be remarked that a low value of generated mechanical specific energy does not imply a low metabolic need. It is possible that the combination of large stresses with low velocities of shortening result in a low efficiency of the contractile mechanism.

Ad 5: Fig. 10.11 shows the simulation of a cardiac cycle which is characterized by a severe coronary artery stenosis. The hemodynamics of the ventricle and aorta are normal. Only coronary perfusion is strongly affected. During diastole the pressure distal to the coronary artery stenosis is approximately $38 \%$ of the diastolic aortic pressure. In the simulation, the perfusion of the subendocardial layers is almost zero. In this situation, one should realize that the whole coronary vasculature is maximally dilated. Therefore, the distribution of coronary flow is less uniform than in the condition of partial vasoconstriction in the subepicardial layers. 


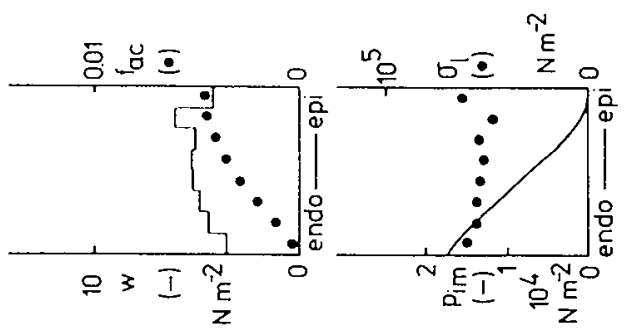

$\frac{5}{0} \sum^{\frac{1}{5}}$

웜

佟

年点

डิ

ช ธิ

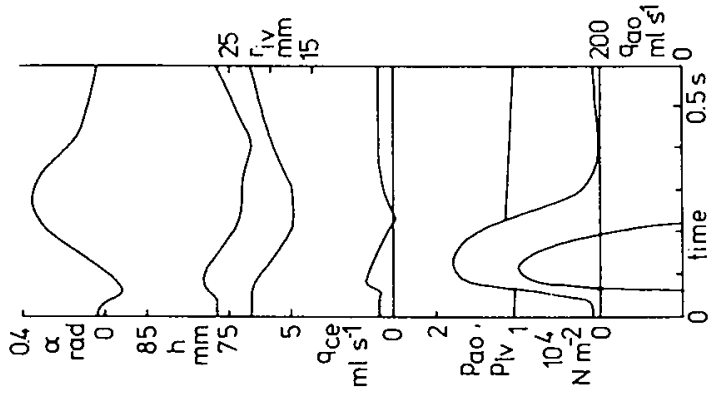

40

동 은 로 总艺 is $\underset{\sim}{\mathbb{N}}$ 구워 -1 0 0ं - $\sim 2 \frac{1}{0}$ 岂吉. 可的出 노용

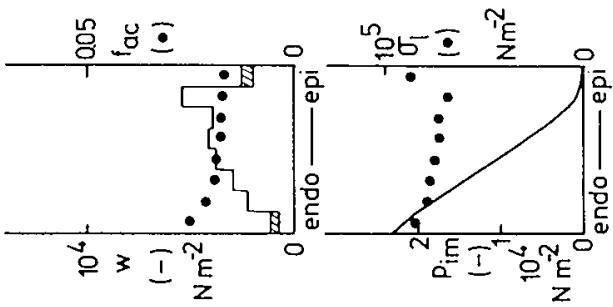

品 = $n$ 동ํㅇ a $-\frac{0}{E}$ उ 0 उ㐫 i 응 4 is ○迈. 두 गु 유 0 4

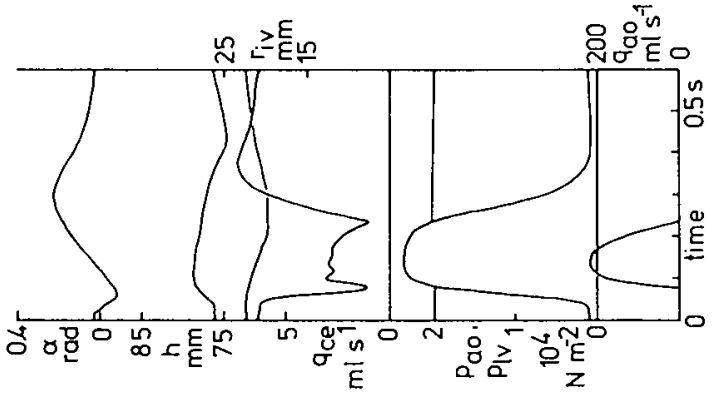

๙ 将衣总 드을 क 0 제 豆导导 in $N$

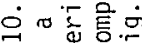
. $4+8$ 000 n 1 舟 幽苾 它引过。

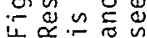




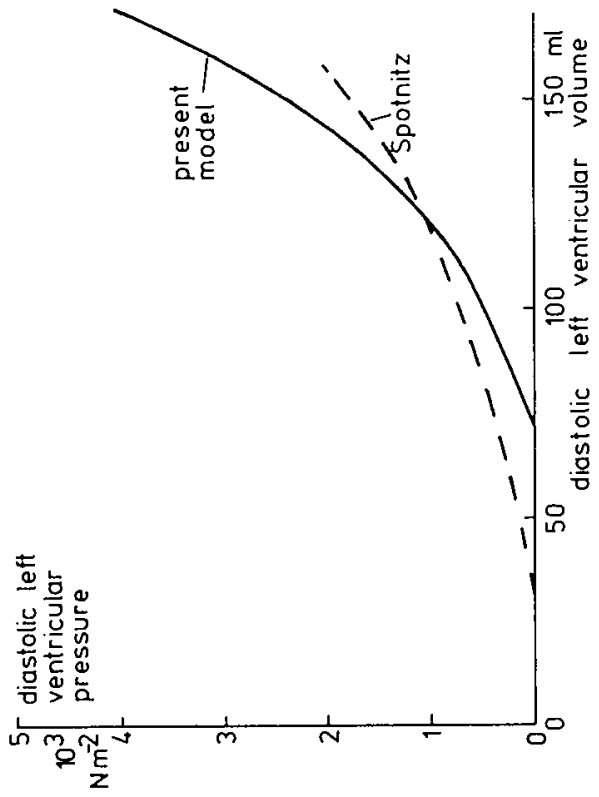

它宓员

证

志岕

$\stackrel{\infty}{2}$

+ $\overline{0}$

पै के a

$5{ }^{2}$

$\because \stackrel{0}{=}$

웅

的品.

宁 的

$1 \pi$

등

बे

등ㅎㅇ

$\cdot 3$

可要导

D $\subseteq 0$

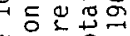

․ㅡㄴ

3 品 $N$

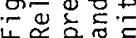

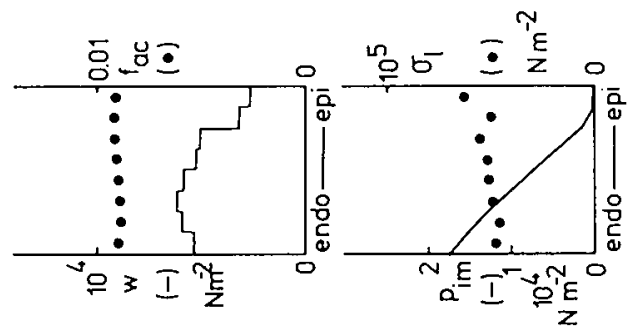

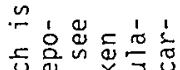

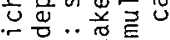
的要政-

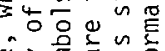
휴을 궈을

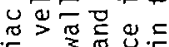

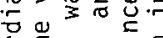

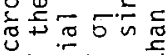

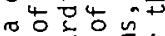

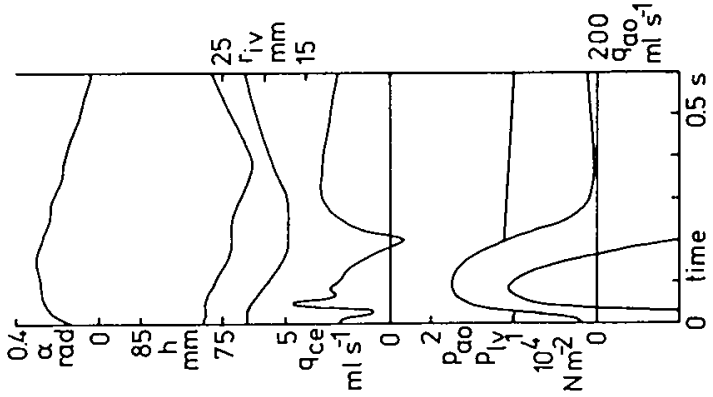

4등 苍号氙 응용요요

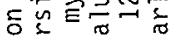

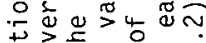

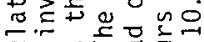

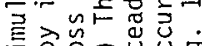
อे n。 $\because$ o d

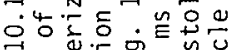

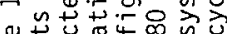

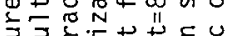

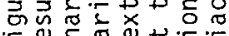

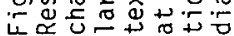


Ad 6: Fig. 10.12 shows the simulation of a cardiac cycle which is characterized by disturbed spread of activation across the myocardial wall. In the clinical situation these disturbances are so complicated that they cannot be simulated by the present model. But qualitatively an impression is obtained by the inversion of the velocity of depolarization across the myocardial wall. In this case, none of the hemodynamic quantities is significantly affected except for the mechanical specific energy, which was significantly larger in the subepicardial layers and significantly smaller in the subendocardial layers, as compared to normal.

Fig. 10.13 presents the diastolic pressure-volume relation of the left ventricle obtained from the data, as presented in the figures 10.2, 10.4 and 10.6 . In the same figure a pressure-volume relation, based on experimental findings of Spotnitz 7 ), is presented. This curve is obtained by changing the volume scale in such a way, that the new left ventricular volume equals $120 \mathrm{ml}$ at a left ventricular pressure of $1000 \mathrm{~N} \mathrm{~m}^{-2}$. 


\section{Literature}

1. Bache, R.J., Mchale, P.A., Greenfield, J.C.: Transmural myocardial perfusion during restricted coronary inflow in the awake dag. Am J Physiol 232 , H645 - H651 (1977).

2. Horwitz, L.D., Bishop, V.S.: Left ventricular pressure-dimension relationships in the conscious dog. Cardiovasc Res 6, 163 - 171 (1972).

3. Myers, W.W., Honig, C.R.: Number and distribution of capillaries as determinants of myocardial oxigen tension. An J Physiol 207 (3), $653-660$ (1964).

4. Pollack, G.H., Krueger, J.H.: Sarcomere dynamics in intact cardiac muscle. Eur J Cardiol 4 , 53 - 65 (1976) Exerpta Medica.

5. Sasayama, S., Franklin, D., Ross, J. Jr.: Hyperfunction with normal inotropic state of the hypertrophied left ventricle. An J Physiol 232, 4418 H425 (1977).

6. Scott Rankin, J., McHale, P., Arentzen, C.E., Ling, D., Greenfield, J.C., Anderson, R.W.: The three-dimensional dynamic geometry of the left ventricle in the conscious dog. Circ Res 39, 304 - 313 (1976).

7. Spotnitz, H.H., Sonnenblick, E.H., Spiro, D.: Relation of ultrastructure to function in intact heart. Sarcomere structure relative to pressure-volume curves of intact left ventricles of dog and cat. Circ Res 18, 49-66 (1966).

8. Streeter, D.D. Jr., Hanna, W.T.: Engineering mechanics for successive states in canine left ventricular myocardium. II. Fiberangle and sarcomere length. Circ Res 33, $656-664$ (1973).

9. Yoran, C., Covel1, J.W., Ross, J.: Structural basis for the ascending limb of left ventricular function. Circ Res 32, 297 - 303 (1973). 


\subsection{Introduction}

In this chapter the results obtained with the model, as presented in the previous chapters, are discussed. Section 11.2 deals with the simulation of a normal cardiac cycle, as represented in fig. 10.2. The time course of left ventricular hemodynamics, mechanical stresses, dimensions and quantities related to the coronary circulation are commented, and discrepancies, as compared with the normal situation are discussed.

In section 11.3 simulations of deviant cardiac cycles, which are presented in section 10.4, are analyzed. In section 11.4 the validity of the model is discussed with respect to several assumptions, which are introduced earlier for mathenlatical convenience. In relation to this, two simulations of normal cardiac cycles, based on deviant mathematical assumptions, are presented and discussed. One simulation is based upon isotropic cardiac muscle properties, which are also discussed in chapter 5 . The other simulation is based upon fixation of the torsion angle at the initial value, which means that torsion of the ventricle along the axis is prevented. Several discrepancies between the model and the animal experiments are likely to have common sources, which are discussed in section 11.5 .

\subsection{Simulation of a normal cardiac cycle}

\subsubsection{Introduction}

This section deals with several aspects of the normal cardiac cycle, as simulated by the model. The definition of a normal cardiac cycle is subject to some arbitrariness since the dynamics of the left ventricle depend on many parameters, which normal value differs from species to species. In the present study the cardiac cycle, shown in fig. 10.2, is presumed to be a simulation of a normal cardiac cycle. Host of the related parameter values are discussed in the previous chapters. Some values of parameters are varied in such a way that the resulting simulation of the left ventricular and aortic pressures and aortic volume flow behave like those quantities in the control situation in the animal experiment, represented in fig. 10.2. In this section the following aspects of the simulated normal cardiac cycle (fig. 10.2) are discussed:

- subsection 11.2.2.: time course of cardiovascular hemodynamics

- subsection 11.2.3.: time course of the geometry of the left ventricle 
- subsection 11.2.4.: transmural course of quantities related to muscle mechanics

- subsection 11.2.5.: time course of coronary artery volume flow and transmural distribution of coronary perfusion.

\subsubsection{Time course of the cardiovascular hemodynamics}

In this subsection the tine course of the left ventricular and aortic pressures and aortic volume flow, as simulated by the model, are discussed. The cardiac cycle starts at the beginning of systole $(t=0)$ with the isovolumic contraction phase. After a short delay ( $=30 \mathrm{~ms}$ ) left ventricular pressure increases rapidly and reaches the aortic pressure. In the simulation the aortic valve opens immediately and aortic volume flow increases rapidly to a maximum of $440 \mathrm{ml} \mathrm{s} \mathrm{s}^{-1}$. Due to the presumed ideal characteristics of the aortic valve (subsection 3.4.1) the pressure difference over the aortic valve is zero and, hence, during the ejection phase aortic pressure follows exactly left ventricular pressure. These pressures reach a maximum of $17500 \mathrm{~N} \mathrm{~m}^{-2}$ and, subsequent$l y$, relaxation of the left ventricle occurs. Left ventricular pressure, aortic pressure and aortic volume flow decrease. When aortic volume flow becomes zero, the aortic valve closes immediately, and the ejection stops. The isovolumic relaxation phase starts. Yet, the aortic pressure is determined by the slow, exponential discharging of the arterial capacitance over the peripheral resistance. The left ventricular pressure decreases rapidly due to relaxation of the cardiac muscle. In the simulation mitral inflow starts halfway the cardiac cycle $(t=300 \mathrm{~ms})$ which results in an increase of left ventricular volume. After a short time, left ventricular relaxation is complete and left ventricular pressure starts to increase slowly due to the increase in ventricular cavity volume. At the end of the cardiac cycle (the end of diastole) left ventricular pressure and volume and aortic pressure have reached the same values as at the beginning of the cardiac cycle. This indicates that, as far as these quantities are concerned, a stationary situation is reached.

The time courses of the left ventricular and aortic pressures and aortic volume flow agree fairly well with the results obtained in animal experiments (fig. 10.3). Some discrepancies of the simulation with the physiological situation are mentioned below.

In the model the relaxation of the left ventricle is slow, as shown by the long tail of the left ventricular pressure curve, This phenomenon may be due to an erroneous slow decay of the presumed time course of the activation of 
the contractile mechanism (chapter 5). Another difference with the animal experiment is the absence of transients in the simulated hemodynamic quantities due to closure of the aortic valve. This is probably caused by the simplifications in the behavior of the aortic valve, which are briefly discussed in subsection 3.4.1. Another discrepancy with the animal experiment is the presence of mitral inflow in the simulation during the relaxation phase of the left ventricle. After mitral inflow has started $(t=300 \mathrm{~ms})$ the simulated left ventricular pressure decreases considerably. This discrepancy may be explained by the non-physiological character of the simulated mitral inflow, which is characterized by a volume flow source in stead of a more realistic pressure source with low internal impedance.

\subsubsection{The time course of the geometry of the left ventricle}

In this subsection the time course of the geometry of the left ventricle as simulated by the model, is discussed. The geometry of the simulated left ventricle is characterized by the inner radius, outer radius and height of the cylinder, representing the inner minor radius, outer minor radius and base to apex distance in the real ventricle, respectively. The torsion angle, which is defined as the angle over which the upper surface of the cylinder (base) is rotated around the axis of the cylinder with respect to the lower surface (apex) of the cylinder, is closely related to the dimensions. Therefore the torsion angle is also discussed in this subsection.

In the simulation the cardiac cycle starts with activation of the subendocardial layers. During the isovolunic contraction phase a significant lengthening of the cylinder occurs, and the inner radius slightly decreases. During the ejection phase both height and inner radius decrease, which is associated with a decrease of the volume of the cavity of the cylinder. After closure of the aortic valve the height and inner radius of the cylinder reach a plateau during the relaxation phase. During mitral inflow ( $t>300 \mathrm{~ms}$ ) the inner radius increases continuously, whereas the height of the cylinder initially decreases and subsequently increases until the end-diastolic dimensions are reached.

In the simulation the torsion angle shows significant changes. At the beginning of the isovolumic contraction phase, before left ventricular pressure increases significantly, the torsion angle decreases. Before opening of the aortic valve, the torsion angle reaches a minimum and subsequently increases approximately linearly during systole. During the relaxation phase this angle 
reaches a maximum and decreases rather rapidly until the diastolic level is reached $(t=450 \mathrm{~ms})$. During diastole the torsion angle slowly decreases to its end-diastolic value. It is remarkable that no discontinuities in the time course of the fiber angle are present when the aortic valve opens or closes.

The increase of the height of the cylinder during the isovolumic contraction phase can be explained as follows. The innermost subendocardial layers are activated first. The fiber angles in those layers are larger than 0.62 rad. $\left(=35^{\circ}\right)$, implying a dominant influence of axial stresses on the geometry. Therefore, initially the height of the cylinder tends to decrease. However, proceeding activation of the myocardium involves contraction of more tangentially directed fibers, which tends to decrease the inner radius and, because of isovolumia of the cylinder cavity, tends to increase the height of the cylinder. Appearently the decrease of the height is insignificant compared to the later occurring increase of this height. This is a result of a dominant influence of the tangential stresses on the geometry of the cylinder in a later stage of the isovolumic contraction phase.

The decrease of the height of the cylinder during the relaxation phase can be explained by a relatively late relaxation of the subepicardial layers, where the fibers are directed more axially than in the other layers of the cylinder. At the end of the relaxation phase, active stresses disappear and the low passive stresses regain their influence on the geometry. This involves rather rapid changes of the geometry towards the diastolic situation. The increase in height and inner radius during diastole is due to filling of the cylinder cavity in this phase of the cardiac cycle.

From the simulation it follows that the time course of the inner radius of the cylinder follows the time course of left ventricular cavity volume, whereas the relative changes in height of the cylinder are smaller and are more influenced by changes in stress distribution across the wall of the cylinder rather than by its cavity volume.

Quantitative data concerning the time course of radius and base to apex distance are presented by Scott-Rankin et al. 17) (fig. 11.1). The increase of the base to apex distance during the isovolumic contraction phase as found by Scott-Rankin ${ }^{17)}(2.1 \%)$ agrees fairly well with the increase in height of the cylinder $(2.3 \%)$ in this phase. Moreover, the decrease of this distance during the ejection phase are also in good agreement (Scott-Rankin: 6.2\%; present mode1: $6.3 \%$ ). The decrease of the inner radius in the experiments of ScottRankin ${ }^{17)}$ is calculated as the increase of the difference between half the 
Figure 11.1

Time course of dimensions of the heart, aortic volume flow and left ventricular pressure in a normal cardiac cycle, as measured by ScottRankin (1976).

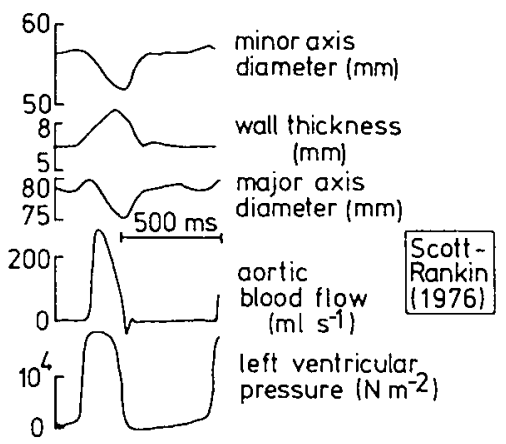

outer minor axis (upper tracing, fig. 11.1) and the wall thickness (second tracing, fig. 11.1). During the ejection phase this decrease was $22 \%$ in the animal experiment (fig. 11.1) and $23 \%$ in the model.

The time course of the torsion angle in the model can be explained as follows. The cardiac cycle starts with activation of the subendocardial layers. Because of the spiral course of the myocardial fibers in these layers around the ventricular cavity in the model, shortening of the subendocardial sarcomeres causes twisting of the ventricle around its axis, which is shown by a decrease of the torsion angle. Due to a reverse spiral course of the subepicardial muscle fibers, these fibers are stretched by this twisting. The decrease of the torsion angle occurs earlier than a significant increase of the left ventricular pressure since deformation of the left ventricle is easely achieved at this stage. Afterall, in this phase the subepicardial layers are activated to a low degree and, therefore, can not bear enough stress to prevent deformation, initiated by subendocardial muscle fibers, which are activated to a much higher degree. In the simulation approximately 60 ms after the start of the cardiac cycle, activation of the myocardial wall has so far developed that left ventricular pressure increases considerably. Then the stresses generated by the endocardial and epicardial sarcomeres counterbalance through the equilibrium of torques in the upper surface of the cylinder. This results in cessation of the decrease of the torsion angle. The approximately linear increase of the torsion angle is related to the rather uniform value of the velocity of sarcomere shortening across the myocardial wall in the simulation. This phenomenon will be discussed in relation to the transmural course of quantities, which are related to muscle mechanics (subsection 
11.2.4). The prolonged high value of the torsion angle during the first part of diastole is probably due to the relatively long period of time before the subepicaridal layers are relaxated completely, and the low, diastolic stresses regain their influence on the geonetry. The slow, approximately linear decrease of the torsion angle during the end-diastolic phase is determined by the passive muscle properties and the increase in volume of the left ventricular cavity. The behavior of the torsion angle in the model cannot be evaluated, since to our knowledge no information is available about the torsion angle in animal experiments.

\subsubsection{Transmural course_of guantities related to muscle mechanics}

In this subsection the transmural course of the values of tensile muscle stress, sarcomere length and generated mechanical specific energy is discussed. These quantities are closely related to changes in dimensions as discussed in the previous subsection 11.2.3. As already discussed in that subsection in the model the cardiac cycle starts with activation of the subendocardial layers. Consequently the sarcomeres are shortened in this layer first, resuiting in torsion of the left ventricle and lengthening of the subepicardial sarcomeres (fig. 11.2). Due to the proceeding activation across the wall, stresses in the myocardium increase, resulting in an increase of the left ventricular pressure in the simulation.

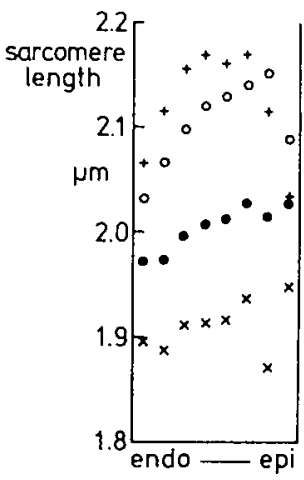

Figure 11.2.

Transmural course of sarcomere length at different moments in the normal cardiac cycle, as simulated by the model. start cardiac cycle, $t=0 \mathrm{~ms}$ - start ejection phase $t=80 \mathrm{~ms}$ middle ejection phase, $t=160 \mathrm{~ms}$ $x$ end ejection phase, $f=220 \mathrm{~ms}$

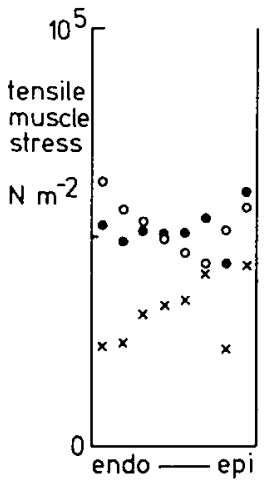

Figure 11.3.

Transmural course of tensile stress at different moments in the normal cardiac cycle, as simulated by the model. 
At the moment, that left ventricular pressure exceeds the aortic pressure, the ejection phase starts and, the left ventricle starts to generate external mechanical power, which is equal to the product of aortic pressure and aortic volume flow during this phase.

At the beginning of the ejection phase transmural differences in tensile stress as well as sarcomere length are small (fig. 11.2 and 11.3). During the whole ejection phase transmural differences in tensile stress and velocity of sarcomere shortening remain smal1. Consequently at the end of the ejection phase transmural differences in generated mechanical specific energy are sma 11. During the relaxation phase small changes in sarconere length occur, due to unequal relaxation of the different layers in the myocardium. Since during the relaxation phase tensile stresses quickly decrease, the changes of generated mechanical specific energy during the relaxation phase and during diastole are negligible ( $<5 \%$ of the total energy per beat).

The small differences in tensile muscle stress during systole across the wall can be explained by the character of the transmural course of the fiber orientation. The transmural course of tensile muscle stress is mainly a result of equilibria of forces and torques in the upper surface of the cylinder, which represents the left ventricle. Layers with a fiber angle close to $\pi / 4$ radians $\left(45^{\circ}\right)$ and close to $-\pi / 4$ radians $\left(-45^{\circ}\right)$ counterbalance through the equilibrium of torques in the upper surface of the cylinder. Layers with mainly axially and tangentially directed fibers counterbalance through to equilibrium of axial forces in the upper surface of the cylinder. In the real ventricle this means that the layers between center and subepicardium counterbalance with the subendocardial layers through the equilibrium of torques, whereas the center layers counterbalance the subepicardial layers through the equilibrium of axial forces. The transmural course of the fiber angle determines the relative thickness of the layers, which are involved in these equilibria and, thus it determines the transmural distribution of tensile muscle stress. In section 10.3 the transmural course of the initial fiber angle is chosen so that the transmural differences in tensile muscle stress are minimum. Since the transmural course of the fiber angle changes to a minor degree during the cardiac cycle, these equilibria of axial forces and torques result in a uniform distribution of tensile muscle stress during the whole systolic period. During diastole low axial forces, borne by the structures in the left ventricular cavity, also influence these equilibria. This results in small transmural differences in tensile muscle stress and, consequently, in small 
transmural differences in sarcomere length during diastole.

Transmural differences in generated mechanical specific energy, as calculated by the model, are sma 71 , which can be explained as follows. At the beginning of the ejection phase transmural differences in sarcomere length and tensile stress are small. Hence, the process of contraction starts under virtually the same conditions for all layers of the myocardium. As already discussed, transmural differences in tensile muscle stress during systole are small, resulting in a similar course of the process of contraction in all layers of the myocardium. After closure of the aortic valve, contraction stops virtually at the same moment in all layers of the myocardium. From this, it may be concluded, that transmural differences of quantities, which are directly related to the process of muscle contraction, j.e. velocity of sarcomere shortening, endsystolic sarconere length (fig. 11.2) and generated mechanical specific energy during a cardiac cycle, are smal1. Hence, the heart seems to be optimally designed, as far as the process of mechanical contraction is concerned. It is not unrealistic to assume that in this situation of mechanical loading the process of contraction is most efficient.

Except for its magnitude, the transmural course of the intramyocardial pressure is rather simular under various conditions because the transmural differences in tensile stress are small. Because of boundary conditions the intramyocardial pressure equals left ventricular pressure at the endocardium and equals zero at the epicardium. Thus, in all layers of the myocardium, intramyocardial pressure is approximately proportional to the left ventricular pressure. At the endocardium the related proportionality factor equals unity, whereas at the epicardium this factor equals zero.

The transmural gradient of the intramyocardial pressure is largest and approximately constant in the subepicardial and center layers, because the fibers in these layers have a smaller radius of curvature than those in the subepicardial layers. The smaller radius of curvature is a result of the more tangential direction of the muscle fibers and the smaller tangential radius of curvature in the subendocardial and center layers. At the subepicardium the transmural gradient of intramyocardial pressure is low. The result is that with increasing distance from the endocardium the intramyocardial pressure decreases approximately linearly until a plateau of low intramyocardial pressure is reached in the subepicardial layers (fig. 10.2). Also as far as restriction to a minimum of the area with high intramyocardial pressure is concerned, the heart seems to be optimally designed. The area of high intramyocardial pres- 
sures is restricted to the inner layers. Layers, which do not contribute directly to the left ventricular pressure, i.e. layers with more axially directed fibers, are situated in the subepicardium ahere intramyocardial pressure is low. As will be discussed in relation to the coronary circulation (subsection 11.2.5), a high intranyocardial pressure is disadvantageous for the coronary perfusion.

Deviations from nomal in the transmural course of tensile muscle stress, generated mechanical specific energy, sarcomere length and intramyocardial pressure are difficult to show, since no reliable experinental information about these quantities has been available. Wall stress has been measured by various investigators $3,5,7,10)$, but transmural differences in this quantity are not considered. Horeover, the technique used influences considerably the local state of mechanical stress, which makes the measurement less reliable ${ }^{7}$.

various methods have been used to measure intramyocardial pressure $2,6,16,20$ ). In an earlier paper ${ }^{1)}$ these methods are discussed with respect to systemic errors due to fiber stresses in the tissue. In all methods the pressure is measured directly or indirectly in a fluid filled cavity surrounded by tissue. The pressure in this cavity does not necessarily equal the intranyocardial pressure. By cutting the muscle fibers, for instance, the fluid filled cavity tends to enlarge, giving rise to a cavity pressure lower than the intramyocardial pressure (fig. 11.4a).

Figure 11.4 .

Artefacts in measuring intramyocardial pressure

(a) cut fibers result in an underestimation

(b) curved fibers result in an overestimation.
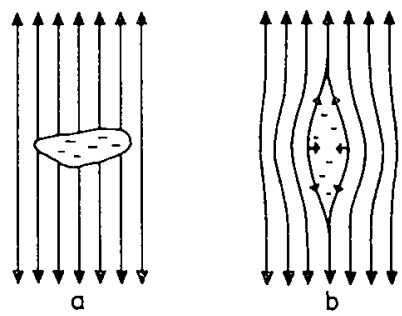

Since stresses in the muscle fibers are nomally much higher than the intramyocardial pressure, even negative pressures can be expected, when the fibers are cut. By disalignment of the muscle fibers (fig. 11.4b) the pressure in the fluid filled cavity tends to be higher than the local intranyocardial pressure as a consequence of Laplace's law, which states that stresses in curved surfaces give rise to pressure gradients. The resulting overestimation is higher at more pronounced curving of the muscle fibers. The conclusion is 
that the pressure in the fluid filled cavity does not only to depend on tire intranyocardial pressure, but also on the stresses in the muscle fibers due to disturbance of the muscle fiber structure.

In the model, the mechanics of the 7 th subcylinder show the largest differ ences compared to the other layers. Stress appears to be lower and velocity of shortening higher. This difference is caused by the relatively large thickness of this layer, as compared to what it should be to eliminate transmural differences in tensile stress. However, in the present model, when assuming 8 subcylinders, the thickness of a layer with a certain fiber angle cannot be smaller than the thickness of one subcylinder. By subdividing the thick walled cylinder into more than 8 subcylinders, this discretization error will be sillaller and the difference can probably be eliminated. However, this procedure has not been carried out.

\subsubsection{Time course of coronary artery volume flow and transinura] distribution of coronary perfusion}

In this subsection the time course of coronary artery volume flow and transmural distribution of coronary perfusion as simulated by the model is discussed. The coronary artery volume flow in the model is the total arterial volume flow, which perfuses the left ventricle. In the physiological situation this volume flow is the sum of the volume flows of all coronary arteries, except for their contribution to the right ventricle. In the animal experiment, good representations, although at a different scale, are the volume flow in the LCC and $L A D C$ because they mainly perfuse the left ventricle. Rather arbitrarely coronary perfusion of the nyocardiun is chosen to be the volume flow from the arteriolar subcompartment into the capillary subcompartment. In the stationary situation, the mean inflow and the mean outflow of each subcompartment within a subcylinder are equal. A slight advantage for choosing capillary inflow as representative of the coronary perfusion is, that this volume flow reaches the stationary situation first.

From the sinulation it is found that the transmural distribution of coronary perfusion is perfectly uniform. This is not amazing, since transmural differences in the coronary microcirculatory parameters are introduced in the model to el iminate transmural differences in coronary perfusion (section 10.3).

At the start of the simulated cardiac cycle, coronary artery volune flow is approximately constant. When the left ventricular pressure increases, co- 
ronary volume flow decreases. After opening of the aortic valve usually a positive peak in this volume flow occurs. After a few strongly damped oscillations, coronary volume flow decreases continuously during the ejection phase, and even becomes negative at the end of this phase. After closure of the aortic valve, coronary perfusion pressure (aortic pressure) is high as compared to intramyocardial pressure and, hence coronary artery volume flow increases to relatively a high level. After reaching a llaximum during diastole, coronary volume flow decreases gradually until the next beat starts and volume flow decreases steeply.

The time course of the coronary artery volune flow, as generated by the model, can be explained as follows. During the isovolumic contraction phase left ventricular pressure increases. As discussed in subsection 11.2.4, the increase of intranyocardial pressure in the subendocardial layers is directly related to this pressure increase. This results in a pressure increase in the subendocardial arteriolar subcompartments and, hence subendocardial perfusion decreases or even stops. This is shown by the decreases of coronary artery volune flow prior to opening of the aortic valve. During the ejection phase two factors determine the net change of coronary artery volume flow, i.e. a decrease in microcirculatory inflow and a change in volume of the large coronary arteries (1.D. > $400 \mathrm{~km})$. The latter effect probably explains the positive peak in coronary artery volume flow at the beginning and the negative peak at the end of the ejection phase. At the beginning of the ejection phase aortic pressure rapidly increases resulting in a rapid increase in volume of the large coronary arteries (I.D. > $400 \mu \mathrm{m})$, due to their capacitance. This explains the positive peak in coronary artery volume flow in this phase. The negative peak at the end of the ejection phase can be explained by the same mechanism because of the decrease in aortic pressure at this moment. Since the decrease of the aortic pressure occurs less rapidly than its increase, the negative peak is smaller in amplitude and it is wider.

Another reason for the decrease in the simulated coronary artery volume flow during the ejection phase is the increasing resistance of the coronary microcirculatory vessels in the subendocardial layers, which is caused by the squeezing effect of the locally high intramyocardial pressure. The latter effect develops probably slower than changes in volume of the large coronary arteries (I.D. $>400$ um).

During the isovolumic relaxation phase in the model, coronary perfusion pressure remains high and intramyocardial pressures decreases. This results 
in an increase in coronary artery volume flow. During diastole the microcirculatory compartments, which were squeezed during systole, are refilled. Therefore, the pressure in the arterioles slowly increases, which partly explains the slow decrease of the coronary artery volume flow during diastole. Another effect, which tends to decrease coronary artery volume flow during diastole, is the decrease of aortic pressure. However, the latter effect can only explain $1 / 3$ of the decrease of coronary artery volume flow during diastole, since the relative decrease of this volume flow during diastole is 3 times the relative decrease in aortic pressure during this phase of the cardiac cycle.

The strongly damped oscillations after the early systolic peak in the coronary artery volume flow are probably an artifact. The inertance and capacitance in the submodel of the large coronary arteries (I.D. $>400 \mu \mathrm{m}$ ) form a circuit with a resonance frequency of approximately $25 \mathrm{~Hz}$. This submodel was specified to be a good approximation of the bahavior of these arteries at frequencies below $15 \mathrm{~Hz}$ (chapter 6). Obviously, at higher frequencies artefacts can be present.

The time course of coronary artery volume flow, as generated by the model, agrees fairly well with the animal experiment (fig. 10.3). Moreover, a qualitative agreement is found with the time course of this flow, as reported in literature $8,9,11,13)$. Quantitative differences, however, are present in these studies. In most cases, these differences can be explained from the insight gained from the present model. For instance, in the recordings of Khouri et al. 9) the increase of the aortic pressure during systole is high and, consequently, a large decrease of the coronary perfusion pressure during diastole is found. This results in a more pronounced decrease of arterial volume flow during diastole, as compared to the situation in the simulation. These larger changes in diastolic aortic pressure probably result from a different aortic input impedance.

The positive peak in coronary artery volume flow at the beginning of the ejection phase is not always present. This peak is a result of two counteracting effects. The more the volume increase of the large coronary arteries and the more rapid it is in early systole the more the peak augments. The peak is counteracted by a decreased microcirculatory inflow, caused by a high intramyocardial pressure at this moment. In some cases, for instance, as found by Khouri ${ }^{9}$ ) in animal experiments, this peak does not exist, probably due to a dominant effect of the decreased microcirculatory inflow. In the case of a simulated hypertension by the model (fig. 10.7) this peak is also 
absent. In this situation, after all, left ventricular pressure is high, resulting in a pronounced decrease of microcirculatory inflow during systole. Moreover the increase in aortic pressure after opening of the aortic valve is moderate, which results in a lower volume increase of the large coronary arteries (I.D. > 400 ; lil) during this phase.

In the animal experiment (fig. 10.3) a short positive peak in coronary artery volume flow occurs immediately after closure of the aortic valve. This peak appears to be related to the pressure fluctuations, which are associated with the closure of the aortic valve. In the simulation the simplification of the behavior of the aortic valve does not allow pressure fluctuations (subsection 11.2.2) and the short positive peak, therefore, does not occur. Moreover, in the animal experiment coronary artery volume flow rapidly reaches its diastolic level, whereas in the simulation this flow is increasing rather slowly to this level. This discrepancy can be explained by slow relaxation of the left ventricle in the model, which results in a prolonged duration of the pressence of high intranyocardial pressures after closure of the aortic valve.

\subsection{Simulations of deviant cardiac cycles}

\subsubsection{Introduction}

The present section deals with simulations of deviant cardiac cycles, as presented in section 10.4. Several characteristics, which also are relevant for the normal cardiac cycle, have been discussed earlier in section 11.2. In the present section special attention is paid to the consequences of deviations of normal cardiac perfornance for the quantities, which are calculated by the present model. Moreover, for some of these deviations, results obtained by the model are compared to those, obtained in animal experiments. In this section the following deviations of a normal cardiac performance are discussed in:

- subsection 11.3.2. Changes in end-diastolic volume and heart rate (fig. 10.4, $10.5,10.6$ and 10.7)

- subsection 11.3.3. Changes in aortic input impedance, which include the simulation of hypertension (fig. 10.10) and aortic occlusion (fig. 10.8 and 10.9).

- subsection 11.3.4. Coronary artery stenosis (fig. 10.11).

- subsection 11.3.5. Disturbed spread of activation across the myocardial wall (fig. 10.12). 


\subsubsection{Changes in end-diastolic volume and heart rate}

In this subsection simulations of cardiac cycles, which are characterized by a large (fig. 10.4), a normal (fig. 10.2) and a small (fig. 10.6) end-diastolic left ventricular volume are discussed. In these simulations the heart rate is chosen to be lower at larger end-diastolic left ventricular volumes. These simulations indicate that, as far as the cardiovascular hemodynamics are concerned, a larger end-diastolic ventricular volume results in:

- a higher end-diastolic left ventricular pressure

- a higher systolic left ventricular and aortic pressure

- a longer duration of the ejection phase.

The relation between end-diastolic left ventricular pressure and volume is presented in fig. 10.13, and is assumed to be determined by the passive elastic properties of the cardiac muscle. An increasing end-diastolic left ventricular pressure as a function of increasing volume is also found in animal experiments as, for instance, reported by Spotnitz ${ }^{18)}$ (fig. 10.13). Fig. 10.13 shows that the left ventricle in the model is two times stiffer than the left ventricle in the animal experiment, as reported by Spotnitz ${ }^{18)}$. This discrepancy is probably due to differences in the passive elastic properties of cardiac muscle in animal experiments and those assumed in the model.

The higher systolic left ventricular and aortic pressures at a higher enddiastolic left ventricular volume, as found in the simulation, is also found in the animal experiment (fig. 10.3, 10.5 and 10.7). In the model the systolic left ventricular pressure is proportional to tensile muscle stress, which will be discussed in relation to the transmural course of quantities, related to cardiac muscle mechanics.

An longer duration of the ejection phase, if end-diastolic left ventricular volume increases is found in the simulation as well as in the animal experiment (fig. 10.3, 10.5 and 10.7). This increase is a result of a larger mean value of the sarcomere length at a larger end-diastolic left ventricular volume. As discussed in chapter 5 , increasing sarcomere length causes a longer duration of the activation of the cardiac muscle.

As far as the geometry of the left ventricle is considered, the model reveals that a larger end-diastolic volume of the left ventricle results in: - a larger end-diastolic value of the height and radius of the cylinder - a smaller end-diastolic value of the torsion angle of the cylinder - a longer duration of the slow, approximately linear change of the inner radius, height and torsion angle during diastole. 
- a less pronounced increase of the height of the cylinder during the isovolunic phase

- a not significantly affected rate of change of inner radius and torsionangle of the cylinder during systole.

The smallertorsion angle of the cylinder at a larger volume of the cylinder cavity during the end of diastole is determined by passive cardiac muscle properties and the transmural course of the fiber orientation. The dependency of the dimensions and torsion angle on the diastolic ventricular volume is shown fairly well by the long diastolic phase in the simulated cardiac cycle, characterized by a large end-diastolic volume $(170 \mathrm{ml}$; fig. 10.4). In this simulation, left ventricular volume increases linearly during diastole, which results in an virtually linear increase of the inner radius and height of the cylinder and a linear decrease of its torsion angle.

A necessary condition for the linear change of the dimensions and torsion angle during diastole seems to be complete relaxation of the cardiac muscle. Using this criterion, it may be concluded, that the duration of the diastole shortens considerably at higher heart rates, and virtually vanishes at a duration of the cardiac cycle of $420 \mathrm{~ms}$ (fig. 10.6). In the animal experiment, however, the process of relaxation is probably more rapid than in the model, which results in a relatively longer duration of the real diastole.

In animal experiments Scott-Rankin et al. 17) lowered the venous pressure, which resulted in a decreased end-diastolic volume and an increased heart rate. They measured radius (half minor axis), wall thickness and base to apex distance and found a decrease of both radius and base to apex distance, like is found in the present model. Moreover, from their animal experiments it can be derived that the systolic rate of shortening of the inner radius is hardly influenced by a moderately decreased end-diastolic left ventricular volume. However, the rate of shortening of the base to apex distance during systole was less at lower end-diastolic left ventricular volumes. This behavior of the changes in dimensions during systole is also shown by the present model. During the isovolumic contraction phase, however, a discrepancy is found. At a lower end-diastolic left ventricular volume the increase of the base to apex distance during the isovolumic contraction phase, as found by Scott-Rankin ${ }^{17}$ ) in animal experiments, is less than calculated in the model. Moreover, during diastole, the time course of the dimensions of the left ventricle, as simulated by the present model, shows discrepancies with the results as reported by Scott-Rankin from animal experiments. This has been discussed before in rela- 
tion to the normal carciac cycle (subsection 11.2.3, fig. 11.1).

As far as the transmural course of quantities related to muscle mechanics is considered, the model reveals that a large end-diastolic left ventricular volunie results in:

- an increased tensile muscle stress in all layers of the myocardium

- practically unchanged small transnural differences in tensile muscle stress

- an increased generated mechanical specific energy in all layers of the myocardium

- practically unchanged small iransmural differences in generated mechanical specific energy

- relatively smaller transmural differences in quantities, related to cardiac muscle mechanics, as compared to their mean value

- practicaliy unchanged transinural course of the intramyocardial pressure, except for a constant factor, which is proportional to the instantaneous value of the left ventricular pressure.

The larger values of tensile nuscle stress and generated mechanical specific energy if end-diastolic volume increases are related to larger sarcomere lengths in the myocardium. As shown in chapter 5 , at a larger sarcomere length a larger stress and more mechanical specific energy can be generated. This larger tensile stress results in a higher left ventricular pressure during systole. The small transmural differences in tensile stress have been discussed earlier in section 11.2 .4 in relation to the simulation of the normal cardiac cycle. From fig. 10.4 and fig. 10.6 it appears that transmural differences in this stress remain small at smaller as well as at larger left ventricular volumes. This supports the theory, that under many conditions tensile muscle stress is constant across the wall because of equilibrium of axial forces and torques in the upper surface of the cylinder. At the beginning of the ejection phase transmural differences in sarcomere length are small for the different values of the end-diastolic volume (fig. 11.5).

Figure 11.5.

Transmural course of sarcomere length early in systole, as simulated with different values of the end-diastolic volume $(t=80 \mathrm{~ms}, \mathrm{fig} .10 .4,10.2$ and 10.6).

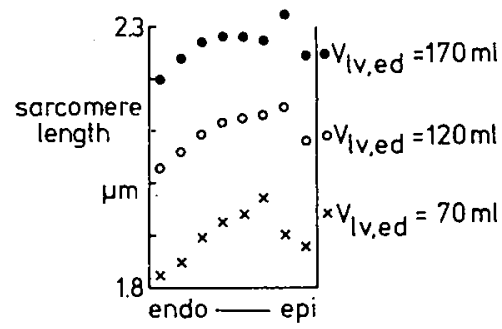


Hence, and also because of small transmural differences in tensile stress, transmural differences in quantities, related to cardiac muscle mechanics are small at different end-diastolic left ventricular volumes.

Experimentally determined local values of the quantities, which are related to cardiac muscle mechanics, have not been reported in literature, as far as known. Therefore, direct verification could not be performed. Some indirect evaluation, however, is possible. Downey et al. ${ }^{4)}$ measured local myocardial perfusion with radioactive microspheres. Under various circumstances of hemodynamic loading of the heart they found a constant perfusion across the myocardial wall. Since in these experiments, the coronary circulation was not completely dilated, it is likely that coronary perfusion is mainly determined by metabolic needs. Assuming, that the metabolic needs of cardiac muscle are the same under similar mechanical conditions, an indication is obtained that in vivo under various circumstances transmural differences in cardiac mechanics are small.

As far as the time course of the coronary artery volume flow and transmural distribution of coronary perfusion is considered, the model reveals that a larger end-diastolic left ventricular volume results in:

- a larger systolic component of the coronary artery volume flow

- a more pronounced decay of coronary volume flow during diastole

- practically unchanged small transmural differences in coronary perfusion.

The systolic component of coronary volume flow is larger if end-diastolic volume increases. This is probably due to the fact that systolic aortic pressure, which equals the coronary perfusion pressure, is relatively higher than the diastolic component of this pressure. This benavior is also found in the animal experiment, presented in fig. 10.5 and 10.7 .

In the simulations the decay of coronary artery volume flow during diastole is more pronounced at larger end-diastolic ventricular volumes, since in that case the duration of the diastole is longer. In the model, relaxation is rather slow, and consequently, the increase of coronary artery volume flow is slow. Thus a relatively short period of time is left for complete diastole, especially at high heart rates. In the simulation the left ventricle is even not completely relaxated at the end of the cardiac cycle at a duration of this cycle of 420 ris (fig. 10.6). In animal experiments, as presented in fig. 10.5 and 10.7 , this behavior is not to be observed. In these experiments no significant decay of the coronary artery volume flow is found during diastole. In animal experiments reported by van der Heer et al. ${ }^{21)}$, such a decay is found. In their experiments, however, hemodynamic changes were induced by 
drugs which can influence heart rate and, therefore, the end-diastolic volume 14). Their changes qualitatively agree with the simulated hypovolumia as presented in fig. 10.6. In the animal experiments the decay of coronary artery volume flow during diastole was shortened and, therefore, less pronounced as compared to normal. Moreover, in this case, the increase of this flow after closure of the aortic valve was slower. Both findings are also shown by the simulation, characterized by a small end-diastolic volume and high heart rate (fig. 10.6), as compared to simulation of the normal cardiac cycle (fig. 10.2).

\subsubsection{Simulation of changes in aortic ingut impedance}

In this section two simulations of cardiac cycles based on changed aortic input impedance are considered, i.e. occlusion of the aorta (fig. 10.8) and increased peripheral resistance (fig. 10.10). Fig. 10.9 deals with a cardiac cycle in the animal experiment after occiusion of the aorta just distal to the aortic valve. With respect to the cardiac mechanics and hemodynamics both simulations are of the same nature. In the situation of increased peripheral resistance, aortic inflow is partly obstructed, whereas in the situation of aortic occlusion, aortic inflow is completely obstructed. The latter is merely a more extreme deviation from normal than increased peripheral resistance. For this reason, with respect to cardiac mechanics and hemodynamics the simulation of aortic occlusion is discussed and the simulation of an increased peripheral resistance is supposed between aortic occlusion and normal. This assumption is supported by the results presented in fig. 10.2 (normal), fig. 10.8 (aortic occlusion) and fig. 10.10 (high peripheral resistance). The coronary circulation, however, shows to be of a different nature in both simulations and will be discussed separately.

The hemodynamics and mechanics in the simulation of an isovolumic contraction (fig. 10.8) appear to be different from normal as to the following aspects:

- an increased left ventricular and aortic pressure

- no aortic volume flow

- a longer duration of the systolic phase

- a larger increase of the height of the cylinder early in systole

- an unchanged decrease of the torsion angle during early systole, but a smaller increase of this angle later in systole

- an increased tensile stress during systole 
- practically unchanged transmural differences in tensile stress during systole

- practically unchanged transmural course of the intramyocardial pressure, except for a constant factor, which is proportional to the instantaneous left ventricular pressure

- a low generated mechanical specific energy

- relatively large transmural difference in generated mechanical specific energy

- a significant stretching of activated muscle

The high left ventricular pressure in the simulation is due to the aortic occlusion and is related to high tensile stresses in the myocardial wall. The longer duration of the systole is caused by a longer duration of the activation of the cardiac muscle, which is due to the larger value of the sarcomere length during systole and the extremely low values of the velocity of sarcomere shortening (chapter 5).

The more pronounced increase of the height of the cylinder during systole is caused by the absence of ejection. Thus the volume of the ventricle does not decrease and, the height of the cylinder reaches a stable, large value during systole. During the relaxation phase the height decreases because by then the geometry is again controlled by small diastolic forces. The deviation of this cardiac cycle from normal starts at the opening of the aortic valve in the normal cardiac cycle. Thus, the minimum of the torsion angle, which occurs earlier, is unaffected. Since during systole there is no change in volume, the increase of the torsion angle is slower in the simulation of the isovolumic cardiac contraction.

During systole tensile muscle stress is larger than normal since velocity of sarcomere shortening is close to zero. Transmural differences in tensile stress are also small in this case, which brings about a normal transmural course of the intramyocardial pressure. This phenomenon has already been discussed in relation to the simulation of the normal cardiac cycle. However, in the case of high tensile stresses and low velocities of sarcomere shortening the uniform distribution of the generated mechanical specific energy is disturbed. Small transmural differences in tensile stress and sarcomere length decide on either shortening or stretching of activated cardiac muscle. Thus, transmural differences in generated mechanical specific energy are the more pronounced, the more the change in volume of the left ventricle is restricted during systole. This phenomenon is demonstrated by the simulation of an aor- 
tic occlusion (fig. 10.8) and, to a lesser degree, by the simulation of a high peripheral resistance(fig. 10.10). The shaded area in fig. 10.8 and 10.10 indicate the loss of mechanical specific energy per beat, due to stretching of activated cardiac muscle. This stretching of activated muscle deteriorates the efficiency of the cardiac contraction.

In animal experiments Sasayama et al. 15) occluded the aorta and found that during systole the inner radius of the left ventricle decreases approximately 20\%. In the simulation this decrease is only $4 \%$. The results of Sasayama et a1., however, are probably not contradictory to the present model, as can be explained as follows. A decrease of the inner radius of $20 \%$ must cause either a decrease of the volume of the left ventricle or an increase of the base to apex distance of approximately $40 \%$. Since the latter is unrealistic, the more so as normal changes are in the order of magnitude of a few percent 17), in their experiment the volume of the left ventricle had to decrease during systole. Hence, it is likely that the related cardiac contraction is not performed under isovolumic conditions.

As far as coronary artery volume flow in a isovolumic cardiac cycle is concerned, the results of the model, as compared to normal, are:

- a positive peak in early systole of practically normal magnitude

- a tremendously augmented systolic coronary artery volume flow.

The augmented systolic coronary artery volume flow is probably due to the large increase of the aortic pressure. During systole, the proximal, subepicardial subcompartments of the coronary microcirculation are rapidly filled, since in the related layers the transmural pressure is highly increased. This rapid filling results a high coronary arterial inflow during systole. However, due to this filling, intravascular pressure increases and, hence, coronary inflow decreases during systole. This is shown by the decrease of coronary artery volume flow, when the aortic pressure is maximum. The animal experiment, in which the aorta is occluded (fig. 10.9), agrees fairly well with the simulation (fig. 10.8) as far as this volume flow is considered. As already discussed in section 10.4, the simulation holds up to the moment of ciosure of the aortic valve in the experimental situation.

The time course of coronary artery volume flow in a simulated cardiac cycle, which is characterized by a high peripheral resistance (fig. 10.10), is significantly different from normal. The diastolic component of this flow is high, which is due to the high diastolic aortic pressure. Early in systole this flow decreases considerably and remains approximately constant 
during systole. The positive peak in this flow immediately after opening of the aortic valve has disappeared, as already has been discussed in relation to the norila? cardiac cycle (subsection 11.2 .5 ). During the relaxation phase the flow increases to its diastolic value. Fig. 10.10 shows that the coronary perfusion of the myocardial wall is considerably augmented, whereas, subendocardial perfusion is slightly higher than subepicardial perfusion. This may be explained as follows. During diastole coronary perfusion is high due to high aortic and low intramyocardial pressures. This diastolic perfusion is slightly larger in the subendocardial layers because of the transmural gradients in microcirculatory parameters. During systole subendocardial intramyocardial pressures are high and reduce the perfusion in that area. Normaliy these two effects cancel, which results in a uniform distribution of the coronary perfusion. In the simulation with high peripheral resistance, however, diastolic perfusion dominates, causing a larger subendocardial perfusion. The results of this simulation are not compared with results obtained in animal experiments.

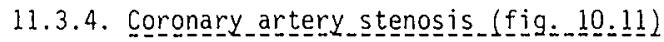

In the model coronary artery stenosis is simulated by an increased resistance of the large coronary arteries (I.D. > $400 \mathrm{um}$; fig. 10.11). In order to avoid mathematical instability of the model the inertance of these arteries is aiso increased. Therefore, the time constant of the inertance-resistance circuit is made larger than the time step, used in solving the system of differential equations according to the Runge Kutta method. As discussed in chapter 4 this system of differential equations is the mathematical base of the present model.

In the simulation of a cardiac cycle, characterized by a coronary artery stenosis of this degree, the hemodynamics and mechanics appear to be normal. Quantities, which are directly related to the coronary circulation, however, are changed considerably. From the simulation it follows as compared to normal, that:

- coronary artery volume flow is significantly smaller

- systolic coronary artery volume flow is not significantly lower than diastolic volume flow

- coronary perfusion is decreased in all layers of the myocardium

- subendocardial perfusion is only $5 \%$ of the subepicardial perfusion, resulting in large transmural differences in coronary perfusion. 
In this simulation the smaller coronary volume flow is caused by the high resistance in the large coronary arteries. This is shown by a low, pressure distal to this resistance (the stenosis) during diastole (38; of the diastolic aortic pressure). The subendocardial perfusion is low since the effective perfusion pressure of the coronary circulation is much lower than the subendocardial intramyocardial pressure during systole. The subendocardial coronary circulation is squeezed during systole and cannot sufficiently be refilled during diastole. This results in a sustained low volume of the subendocardial coronary circulation, implying a high subendocardial microcirculatory resistance. On the other hand in the subepicardium the resistance is approximately normal and, therefore, steals subendocardial perfusion flow. The difference between the systolic and diastolic component of the coronary artery volume flow is small since coronary perfusion is mainly determined by perfusion of the subepicardial layers, where intramyocardial pressures are low. Noreover, the largest pressure drop in the coronary circulation occurs in the large coronary arterial resistance and, consequently, changes of the resistance in other parts of the coronary circulation have a minor influence on the coronary artery volume flow. This behavior of the coronary artery volume flow is also found in the recordings of Khouri ${ }^{9}$ ).

\subsubsection{Disturbed spread_of_activation_across the myocardial_wa]!_f}

A qualitative impression of the consequences of a disturbed spread of activation across the myocardial wall is obtained by inversion of the velocity of depolarization. In the simulation the depolarization wave front travels from the epicardium to the endocardium which, obviously, is a non-physiological situation.

From the simulation it appears that no significant changes in cardiac and coronary hemodynamics are present. Cardiac performance seems not to be affected by this disturbance. However, significant differences, as compared to the simulation of the normal situation, are found in the time course of the torsion angle and the transmural distribution of the generated mechanical specific energy. This can be explained as follows. In this situation cardiac contraction starts at the subepicardium, implying early shortening of subepicardial sarcomeres. Twisting of the ventricle occurs in a direction opposite to normal, which results in an increase of the torsion angle and the subendocardial sarcomere length (fig. 11.6). During the ejection the distribution of tensile stress is uniform like in all simulations. However, the sarcomere 
Figure 11.6 .

Transmural course of sarcomere length early in systole, as simulated with normal and negative velocity of the depolarization wave front (nomal: $t=80 \mathrm{~ms}, \mathrm{fig}$. 10.2; negative: $t=$ 40 ms, fig. 10.12).

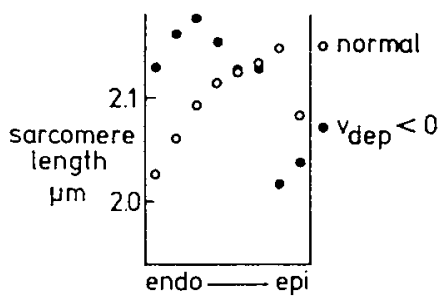

length is larger in the subendocardial layers than in the subepicardial layers. This results in a larger value of the generated mechanical energy in the subendocardial layers than in the subepicardial layers, which is shown by fig. 10.12. The conclusion is that disturbed activation may cause large differences in generated mechanical specific energy, whereas cardiac performance may be practically normal. The heart probably contracts less efficiently than under normal circumstances.

11.4. Validity of the model with respect to mathematical properties and assumptions

In this section the validity of the model is discussed with respect to some mathematical properties and assumptions. The validity of the mechanics of the left ventricle in the model is tested by means of the balance of energy, as presented in section 8.3 . The sum of the punping energy, generated by the left ventricle and the energy loss in the coronary circulation due to squeezing must be equal to the total mechanical energy, generated by the cardiac muscle in all subcylinders.

In the present model the torsion angle may change during the cardiac cycle. In an earlier stage of the present study this torsion angle was fixed for mathematical convenience. With that assumption tangential displacements and the equilibrium of torque have not to be considered. In this section a normal cardiac cycle is simulated under the condition that the torsion angle is fixed, and compared with the simulation of a normal cardiac cycle with freely varying torsion angle (fig. 10.2).

In chapter 5 the assumption of isotropic myocardial material is discussed. In a concept of a thin-walled cylinder, representing the left ventricle, this assumption is rejected for reason of unrealistic stiffness which results for the left ventricle. As another check of this assumption, a cardiac cycle is 
siriulated, in which isotropic liluscle material properties are introduced in the model. The resulting hemodynamics are compared with the simulation of a norilal cardiac cycle, based on anisotropic myocardial material.

The check on the balance of energy is performed in the simulation of a normal cardiac cycle, represented in fig. 10.2. The pumping energy $\left(w_{s}\right)$, which is generated by the left ventricle during one cardiac cycle, is calculated according to relation (8.3.4):

$$
W_{S}=0.795 \mathrm{~J}
$$

The mechanical energy $\left(W_{m}\right)$, generated by the cardiac muscle, is computed according to relation (8.3.3). For this purpose in each subcylinder the muscle volume is multiplied by the mechanical specific energy, generated during one cardiac cycle. After adding the contributions of all subcylinder the generated mechanical energy is found to be

$$
W_{\mathrm{m}}=0.801 \mathrm{~J}
$$

The energy loss $\left(W_{c}\right)$ due to squeezing of the coronary circulation is calculated by an approximation of equation (8.3.5). Since the contribution to this energy loss is only significant during systole, and even then is much smaller than the contribution to $W_{n}$, the energy loss is calculated with a sufficient accuracy by the approximation

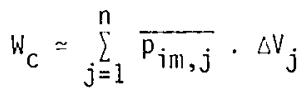

where $\overline{p_{i m, j}}=$ mean intramyocardial pressure in subcylinder $j$ during systole $\Delta V_{j}=$ volume change of the coronary circulation in subcylinder $j$ during systole

Relation (11.4.3) renders for the simulation of the normal cardiac cycle (fig. 10.2):

$$
W_{C}=0.0057 \mathrm{~J}
$$

This energy loss is small as compared to the total mechanical energy $W_{m}$. However, in the inner subcylinder, where squeezing is most pronounced, the energy loss due to squeezing is approximately $6 \%$ of the generated mechanical en- 
ergy in that subcylinder. From relations (11.4.1), (11.4.2) and (11.4.4) it follows that the relation, which expresses the balance of energy

$$
w_{S}+w_{C}=w_{m}
$$

is satisfied quite accurately. This validation indicates that calculations, related to the mechanics and hemodynamics of the ventricle are likely to be correct, as far as the mathematics are concerned.

The simplification, that the torsion angle is fixed during the cardiac cycle is investigated by simulation of a cardiac cycle, which is characterized by such fixation of the torsion angle (fig. 11.7). The results are compared with the normal simulation of the nomal cardiac cycle (fig. 10.2). Apparently the influence of fixation of the torsion angle on most hemodynamic quantities is moderate. Even transmural differences in generated mechanical specific energy are small. However, during the ejection phase, twisting of the ventricle is absent and, therefore, the velocity of sarcomere shortening in the endocardium is significantly higher (approximately 2 times) than in the subepicardium. The larger subendocardial velocities of sarcomere shortening involve a lower tensile muscle stress in the subendocardial layers. In this way, transmural differences in generated mechanical specific energy are as low as in the normal simulation of a normal cardiac cycle (fig. 10.2). However, because of the lower tensile stresses in the subendocardium, the largest transmural gradients in intramyocardial pressure are shifted towards more epicardial situated layers. This implies that a larger volume of cardiac muscle is loaded with large systolic values of the intramyocardial pressure. A free torsion angle is probably closer to the real situation, since no strong enough structures of fixation of the torsion angle are known and the fixation results in larger transmural differences in muscle mechanics. It should be remarked, that the right ventricle would be able to fixate the torsion angle of the left ventricle to a minor degree. For the complete myocardium, however, the assumption of a free torsion angle must be valid. No external structures are present, which are able to prevent, even to a minor degree, free rotation of the apical side of the heart.

The assumption of isotropic material properties of the myocardium is checked at an early stage of the present study. This assumption decides on the validity of the model. Therefore, a simulation of a normal cardiac cycle based on isotropic material, is generated by the model, and compared to a 
Figure 11.7 .

Comparison of the left ventricular pressure and aortic volume flow tracings as obtained from simulations with isotropic and anisotropic properties of myocardial tissue.

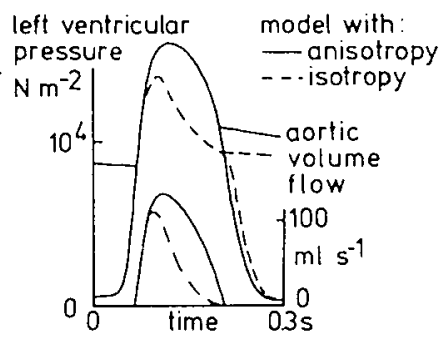

simulation based on anisotropic properties of cardiac muscle (fig. 11.7). It should be renlarked that in this simulation the torsion angle was fixed, however, this has practically no influence on the cardiovascular hemodynamics. In fig. 11.7 it is shown, that an isotropic cardiac muscle results in a nonphysiological exponential decrease of the aortic volume flow during the ejection phase. Horeover, the fast rise and practically exponential decrease of the left ventricular pressure also are not realistic. The findings, reported in section 5.7 , that the systolic isotropic left ventricie is stiffer than normal, is supported by the results of the present simulation. This can be explained as follows. The time constant, which is related to the process of ejection, is approximately equal to the product of the left ventricular systolic capacitance and the characteristic impedance of the aorta. In the present simulation (fig. 11.7) the related capacitance is that small, that the time constant is in the order of magnitude of the duration of the ejection phase. THis is shown by the exponential decline of the left ventricular hemodynamic quantities. Another consequence of this stiffer behavior of the systolic left ventricle is the relatively low ejection fraction (18\%), as compared to the normal simulation $(42 \%)$. The conclusion is that the process of ejection cannot be adaquately described when assuming isotropic myocardial material properties.

\subsection{Sources of discrepancies of the model with the animal experiment}

In this chapter several discrepancies are mentioned in relation to the simulations of either a normal cardiac cycle (section 11.2) or deviant cardiac cycles (section 11.3). Several of these discrepancies are related to a common source. In the present section subsequently the following sources are discussed:

- possible influence of the right ventricle

- possible influence of structures in the ventricular cavities 
- uncertainties related to cardiac muscle properties

- simplification of the aortic valve

- uncertainties in parameters belonging to the coronary circulation.

In chapter 3 the possible influence of the right ventricle is discussed, and it is concluded that if the right ventricle is disregarded no large errors are introduced. However, a possible influence of the right ventricle on the left ventricle, which to our knowledge has not been discussed before, is its influence on the equilibrium of torques and axial forces. These influences are indicated by the large influence of the 7 th subcylinder on the equilibrium of torque in the model, as well as in discrepancies in the transmural course of the fiber orientation between the model and the in vivo situation 19)

In the model it was found, that the systolic tensile muscle stress in the 7 th subcylinder was sinaller than in the other subcylinders. Despite this low stress, the torque, delivered by the 7 th subcylinder alone, counterbalances practically completely the sum of the torques, delivered by the two subendocardial subcylinders. This can be explained by the fact, that a subcylinder can bear a torque, which is proportional to the square of its radius (relation 7.4.4). When looking at the cross-section of the myocardium (fig. 2.2), the right ventricular wall can be regarded as a segment of a subcylinder with a large radius. This implies, that its influence on the equilibrium of torques may be considerable. In an analogeous way, it is indicated that the influence of the right ventricular wall on the equilibrium of axial forces may a) so be important. In section 10.2 and 10.3 it is discusses that in the model the equilibria of torques and forces in the upper surface result in non-physiological changes of dimensions of the ventricle, when using experimental data on the transmural fiber orientation, as obtained by streeter ${ }^{19}$ ). Therefore, the transmural course of the fiber orientation has been changed. By inserting the right ventricle into the model, this change of fiber orientation might not be necessary.

The ventricular cavities contain various structures such as papillary muscles, which are attached to the mitral and tricuspid valves through the chordae tendinae, and fibrous tissue at the endocardium. The influence of all these structures is simplified to an axially directed passive elastic element. This element has to be inserted into the model to simulate the phenomenon that the diastolic geometry is different from the systolic geometry, when the volume is the same in both situations. Probabiy this simpification 
is too crude. However, improvenent of this aspect of the model is difficult since quantitative data about the influence of these structures on the geometry are not availabie yet.

Froli fig. 10.13 it appears that in the model the ventricle during diastole is approximately two times stiffer than in the animal experiment, as reported by Spotnitz ${ }^{18)}$. The difference cannot be explained by a more isotropic behavior of passive elastic muscle, since this would even result in a stiffer behavior of the ventricie in the model. A possible explanation for this stiffness is the different elastic properties of the passive cardiac muscle in both situations. Data, used in the model, are obtained from iin vitro experiments at temperatures of 20 to $30^{\circ} \mathrm{C}$ on cat, rabbit and rat papillary muscle. Moreover, most of these experiments are performed with the use of an experimental set-up, where non-uniform stretching of the muscle under investigation could occur easily ${ }^{12}$ ). It is not unlikely that passive elastic properties of in vivo myocardial nuscle of a dog at a temperature of $37^{\circ} \mathrm{C}$ are different. Although a correction for the temperature is made, the same remark can also be made with respect to the active properties of cardiac muscle. Probably the process of relaxation of the cardiac muscle is too slow in the simulation as compared to the animal experiment. Such a slow relaxation of the cardiac muscle in the model can explain the following discrepancies with the animal experiments:

- a slower decay of the left ventricular pressure during the relaxation phase - a longer period of time required for regaining the diastolic dimensions of the left ventricle

- a longer period of time required for reaching maximum diastolic coronary artery volume flow, which probably results from a slower decay of the intramyocardial pressures.

The nature of the cardiac contraction looks physiological, but possibly some values of related parameters have to be reconsidered to obtain a more accurate discription of the cardiac contraction.

The simplification of the behavior of the aortic valve (section 3.4.1) probably cause some differences. In the model closure of the aortic valve does not result in aortic backfiow and transients in the aortic pressure. Since the aortic pressure is the perfusion pressure of the coronary circulation, transients in coronary artery volume flow also do not appear in the model after closure of the aortic valve. In the animal experiment (fig. 10.3) these transients are present, as shown by the high, positive peak of this volume 
flow after closure of the valve.

As already mentioned in the chapter 6 and 9 , the values of the parameters belonging to the coronary microcirculation probably are not quite reliable. This is due to the large number of related parameters in the model, as well as to the little information about the values of these parameters. In the animal experiment, the time course of the coronary artery volume flow appears to be different from species to species. Discrepancies are difficult to show because the model also shows large variations at different values of the microcirculatory parameters. 


\section{Literature}

1. Arts, T., Reneman, R.S.: Analysis of intramyocardial pressure (IMP) a model study. 9th European Conference on Microcirculation, Antwern 1976. Bibl Anat 15, 103 - 107 (1977).

2. Baird, R.J., Manktelow, R.T., Cohoon, W.J., Williams, W.G., Spratt, F.. H.: Improved pressure gradients and flow rates in myocardial vascular implants. Ann Surg 168, $736-749$ (1968).

3. Burns, J.W., Covell, J.W., Myers, R., Ross, J.: Comparison of directly measured left ventricular wall stress and stress calculated from geometric reference figures. Circ Res 28, 611 - 621 (1971).

4. Downey, H.F., Bashour, F.A., Boatwright, R.B., Parker, P.E., Kechejian, S.J.: Uniformity of transmural perfusion in anaesthetized dogs with maximally dilated coronary circulations. Circ Res 37, 111 - 117 (1975).

5. Feigl, E.O., Simon, Fry, D.L.: Auxotonic and isometric cardiac force transducers. J Appl Physiol 23, $597-600$ (1967).

6. Guyton, A.C.: A concept of negative interstitial pressure based on pressures in implanted perforated capsules. Circ Res $12,399-414$ (1963).

7. Huismans, R.M.: Forces in the wall of the left ventricle. Thes is Free University Amsterdam (1977).

8. Khouri, E.M., Gregg, D.E., Rayford, C.R.: Effect of exercise on cardiac output, left coronary flow and myocardial metabolism in unanesthetized dog. Circ Res 17, $427-437$ (1965).

9. Khouri, E.M., Gregg, D.E., Lowensohn, H.S.: Flow in the major branches of the left coronary artery during experimenta? coronary insufficiency in the unanesthetized dog. Circ Res 23, $99-109$ (1968).

10. McHale, P.A., Greenfield, J.C.: Evaluation of several geometric models for estimation of left ventricular circumferential wall stress. Circ Res $33,303-312(1973)$.

11. Pitt, B., Gregg, D.: Coronary hemodynamic effects of increasing ventricular rate in the unanesthetized dog. Circ Res 22, $753-761$ (1968).

12. G.H. Pollack, Krueger, J.W.: Sarcomere dynamics in intact cardiac muscle. Eur J Cardiol 4,53-65 (1976) Exerpta Medica.

13. Reneman, R.S., Spencer, M.P.: The use of diastolic reactive hyperemia to evaluate the coronary vascular system. Ann Thorac Surg 13, $477-487$ (1972).

14. Rushmer, R.F., Finlayson, B.L., Hash, A.A.: Shrinkage of the heart in anesthetized dogs. Circ Res 2, 22 - 27 (1954).

15. Sasayama, S., Franklin, D., Ross, J. Jr.: Hyperfunction with normal inotropic state of the hypertrophied left ventricie. Am J Physiol 232, $H 418$ - H425 (1977). 
16. Scholander, P.F., Hargens, A.R., Hiller, S.L.: Negative pressure in the interstitial fluid of animals. Science 161, $321-328$ (1968).

17. Scott Rankin J., McHale. P., Arentzen, C.E., Ling, D., Greenfield, J.C., Anderzon, R.W.: The three-dimensional dynamic geometry of the left ventricle in the conscious dog. Circ Res 39, $304-313$ (1976).

18. Spotnitz, H.M., Sonnenblick, E.H., Spiro, D.: Relation of ultrastructure to function in intact heart. Sarcomere structure relative to pressurevolume curves of intact left ventricles of dog and cat. Circ Res 18, 49 - $66(1966)$.

19. Streeter, D.D. Jr., Hanna, W.T.: Engineering mechanics for successive states in canine left ventricular myocardium. II. Fiberangle and sarcomere length. Circ Res 33, $656-664$ (1973).

20. Van der Meer, J.J., Reneman, R.S., Schneider, H., Wieberdink, J.: A technique for estimation of intramyocardial pressure in acute and chronic experiments. Cardiovasc Res $\underline{4}, 132$ - 140 (1970).

21. Van der Meer, J.J., Reneman, R.S.: The relation of intramyocardial pressure to coronary bloodflow. Bibl Anat 11, 151 - 157 (1973). (7th European Conference on Microcirculation, Aberdeen). 


\section{SUMMARY AND CONCLUSIONS}

The present mathematical model of the dynamics of the left ventricle and its coronary circulation is composed of several submodels. Each submodel is a simulation of a subsystem such as geometry, left ventricular mechanics, myocardial material properties including the process of contraction, aortic input impedance, large coronary arteries (I.D. > $400 \mu \mathrm{m}$ ) and coronary microcirculation. Most of the parameters, which are involved in such a submodel, have a physical meaning and are clearly and directly related to physiological quantities. The values of these parameters were obtained, when possible, from animal experiments, which were performed to study the related subsystems separately. After composition of the complete model from the submodels, a simulation of the cardiac cycle was performed (fig. 10.1). Although the model showed several discrepancies with the normal cardiac cycle, (section 10.2), many quantities in the simulation had a physiological appearance, such as time courses of aortic volume flow and pressure, magnitude of left ventricular pressure, ejection fraction and, qualitatively, the behavior of the coronary artery volume flow. This indicates that the structure of the model is reasonably correct.

Thereupon the values of several parameters were changed to fit the results of the model to the results of animal experiments. As discussed in chapter 1 , this way of handling has some disadvantages. Discrepancies may be met by changing values of less relevant parameters, which results in new discrepancies. Moreover, systematic errors in the set-up of the model can be disguised. If such errors are present in the model, the validity of the complete model deteriorates. Therefore, the number of parameters, which values are adapted, is kept as low as possible, and on purpose, less significant differences with the animal experiment are not corrected by changing values of related parameters. Adaptation of parameter values can readily be done, since the physical meaning of the parameters is known. The adapted parameters were related to the time course of the activation of cardiac muscle and to the transmural course of fiber orientation, sarcomere length and coronary microcirculatory volume. Then, a second simulation of a normal cardiac cycle was generated (fig. 10.2). The resulting time courses of left ventricular pressure, aortic pressure, aortic volume flow, left ventricular dimensions and coronary artery volume flow agreed fairly well with the findings in anima? experiments, especially with respect to their behavior during systole.

Next, interventions in cardiac performance were simulated by changing 
values of the appropriate parameters. The results of the simulations of several incerventions agreed fairly well with experimental findings, as far as left ventricular pressure, aortic pressure and volume flow, coronary artery volume flow and transmural distribution of coronary perfusion flow were considered. However, as discussed in section 11.5, several discrepancies were found. The sources of these discrepancies are probably related to leaving out of consideration the right ventricle and some other structures of the heart, uncertainties in mechanical properties of cardiac muscle and coronary microcirculation. Also the aortic valve in the model is greatly simplified.

From the model it appears that the torsion angle of the left ventricle is very sensitive to transmural differences in cardiac muscle mechanics. As far as known, the time course of this angle has never been measured in animal experiments. From the present model, it is concluded that as to cardiac mechanics, measurement of this angle would be very helpful to study transmural differences in cardiac muscle performance. This conclusion can be illustrated by the following example. As a result of a severe coronary artery stenosis or occlusion the mechanical performance of the heart deteriorates 3 ). From the simulation of a cardiac cycle under these circumstances it appears that critical coronary perfusion levels are first reached in the subendocardial layers of the left ventricle. This finding is supported by animal experiments ${ }^{1,2)}$. By recording the torsion angle of the left ventricle in such experiments it could be investigated which layers of the myocardium are responsible for the decrease in pumping ability of the left ventricle. Obviously, the present model would be helpful in interpreting the measured changes of the torsion angle.

In adapting the transmural course of the fiber angle and sarcomere length the hypothesis is made that there are only little transmural differences in tensile muscle stress. This hypothesis results in a concept of the heart which appears to be insensitive to various changes in hemodynamic loading, as far as transmural differences in quantities related to cardiac muscle mechanics and coronary perfusion are concerned. On the basis of this finding it is concluded that the heart, including the right ventricle, may be designed in such a way that the above mentioned hypothesis holds for the complete heart.

In this study cardiac muscle was assumed to be anisotropic. The muscle material was considered to consist of a fiber structure embedded in a soft, incompressible material. However, when applying isotropy of cardiac muscie, the results of the model did not agree with those in animal experiments. The ventricle showed to be too stiff. From this it is concluded that models of the 
heart, based on isotropy of the wall material, are not realistic.

A more general conclusion is, that the present model is a useful tool in understanding the dynamical behavior of the left ventricle and its coronary circulation. Relations between hemodynamics, changes in dimensions, and the transmural course of quantities which are related to mechanics of cardiac muscle material and coronary perfusion, are quantified. This quantification gives the opportunity to study relatively complex consequences of several interventions and deviations from normal. This general conclusion is the base of several proposed applications of the present model, such as:

- simulations of interventions in the cardiac cycle

- studies on the subsystems of the heart in viv

- study of the transmural course of quantities, which are related to cardiac mechanics and coronary perfusion

- understanding of clinical deviations in cardiac performance

- education.

Examples of simulations of interventions in the normal cardiac cycle are shown in chapter 10 and discussed in chapter 11 . These simulations have proved to be usefut in understanding the consequences of these interventions for cardiac performance. Moreover, values of some parameters of the model may be determined more accurately by comparison of the results of the simulations with those obtained in animal experiments under comparable circumstances.

The model links the behavior of subsystems of the heart to the general bchavior of the heart. Information about these subsystems can be obtained by experiments on the intact animal. As to this purpose parameters, related to the subsystem to be studied, are varied until the results of the simulation of the cardiac cycle agree with the results obtained in animal experiments. For instance, by measuring the hemodynamic quantities and some dimensions of the heart in animal experiments, information can be obtained about the in vivo contractile behavior of cardiac muscle material. The data thus obtained can be compared with data, obtained from in vitro studies on properties of cardiac muscle, as far as these data are avajlable.

The present model calculates transmural courses of quantities related to cardiac mechanics and coronary perfusion. In studying these quantities, the results, obtained from the model can be compared with the results obtained from animal experiments. An example of such an application is given in this section in relation to the significance of the torsion angle for the transmural course 
of quantities related to cardiac muscle mechanics.

The clinical significance of the present model is a better understanding of normal and deviant cardiac performance. May be in some clinical situations criteria can be given for optimal treatment. For instance, the present model may be helpful to answer the question: "Which heart rate and hemodynamic loading are optimal to obtain sufficient coronary perfusion of all layers of the heart, when a coronary stenosis is present?".

For educational purpose the present model may be helpful in showing the relations between different quantities, which are related to the process of cardiac contraction and the coronary circulation. By programining the present model on a computer in such a way, that it is accessible for medical students, consequences of interventions such as changes in hemodynamic load, contractility of cardiac muscle and many others, can be visualized directly.

\section{Literature}

1. Griggs, D.M., Tchokoev, V.V., Chin chi Chen: Transmural differences in ventricular tissue substrate levels due to coronary constriction. Am J Physiol 222, $705-709$ (1972).

2. Pollack, J.W., Krueger, J.W.: Sarcomere dynamics in intact cardiac muscle. Eur J Cardiol 4, 53 - 65 (1976) Exerpta Medica.

3. Schaper, W.K.A.: The collatoral circulation of the heart. North-Holland Pbl. Co., Amsterdam, Netherlands (1971). 
The relation between tangential wall tension and internal pressure in a thinwalled rotational ellipsoid

The thin-walled rotational ellipsoid is characterised by the major axis $d_{2}$ and the minor axis $d_{1}$, which equals double the equatorial radius $o_{1}$ (fig. 3.4 ). Point $O$ is defined at the equator. The radius of curvature of the elipsoid in 0 in a plane through 0 and the axis of rotation equals $\rho_{2}$. According to Laplace's law the pressure difference $p$ over the ellipsoidal wall equals

$$
p=\frac{T_{1}}{p_{1}}+\frac{T_{2}}{p_{2}}
$$

where $T_{1}=$ the tangential wall tension in $0\left(\mathrm{~N} \mathrm{~m}^{-1}\right)$

$$
T_{2}=\text { the axial wall tension } 0 \quad\left(\mathrm{~N} \mathrm{~m}^{-1}\right)
$$

For the total force $F_{a}$ acting in the equatorial cross-section of the ellipsoid into the axial direction it holds:

$$
F_{a}=\pi d_{1} T_{2}
$$

Because of equilibrium forces in the top half of the ellipsoid the force $F_{a}$ equals the force acting on the cross-sectional surface due to the pressure $p$. This results in the equation

$$
F_{a}=\frac{\pi}{4} d_{1}^{2} p
$$

Elimination of $F_{a}$ from the equations (A 3.1.1) and (A 3.1.2) results in the following relation for $\mathrm{T}_{2}$

$$
\mathrm{T}_{2}=\frac{\mathrm{pd}}{4}
$$

Substitution of (A 3.1.3) into (3.2.1), and using $d_{1}=2 p_{1}$, results in

$$
T_{1}=\left(1-\frac{\rho_{1}}{2 \rho_{2}}\right) p \rho_{1}
$$




\section{Left ventricular capacitance during systole}

The capacitance of the left ventricle is calculated on the base of wall material properties. The left ventricle is simulated by a thin-walled cylinder. The average of inner and outer radius is denoted as $r$, the volume enclosed by the mid-wall cylinder is $V_{c}$ and the wall thickness is $h$. The modulus of elasticity $(H)$ is defined according to relation (5.7.1). The myocardial material is supposed to be incompressible. The systolic capacitance of the left ventricular cavity is defined in section 5.7. In section A 5.1 and section A 5.2 the capacitance is computed in the case of isotropic as well as anisotropic material properties.

A 5.1. Capacitance of the left ventricle, consisting of isotropic material, $\left(\mathrm{C}_{1 \mathrm{v}}^{\text {is }}\right.$; fig. A 5.1)

In a thick walled isotropic incompressible cylinder the radial, tangential and axial components of the stress $\sigma_{r}, \sigma_{t}$ and $\sigma_{z}$ respectively are described by

$$
\begin{aligned}
& \sigma_{r}=p \frac{r_{1}^{2}}{r_{2}^{2}-r_{1}^{2}}\left[1-\frac{r_{2}^{2}}{r_{c}^{2}}\right] \\
& \sigma_{t}=p \frac{r_{1}^{2}}{r_{2}^{2}-r_{1}^{2}}\left[1+\frac{r_{2}^{2}}{r_{c}^{2}}\right] \\
& \sigma_{z}=p \frac{r_{1}^{2}}{r_{2}^{2}-r_{1}^{2}}
\end{aligned}
$$

where $r_{1}=$ the inner radius of the cylinder

$r_{2}=$ the outer radius of the cylinder

$r_{c}=$ the radial coordinate inside the cylinder

$p=$ the pressure inside the cylinder.

The outer pressure is supposed to be zero. After substituting

$$
r=\frac{r_{1}+r_{2}}{2}
$$


and

$$
h=r_{2}-r_{1}
$$

into the relations ( $A$ 5.1.1), (A 5.1.2) and (A 5.1.3), subsequently averaging the stresses over the cross-sectional areas followed by neglecting the second order terms with $\left(\frac{h}{r}\right)^{2}$ results into

$$
\begin{aligned}
& \sigma_{r}=p \frac{r}{h} \cdot\left\{-\frac{1}{2} \frac{h}{r}\right\} \\
& \sigma_{t}=p \frac{r}{h} \cdot\left\{1-\frac{1}{2} \frac{h}{r}\right\} \\
& \sigma_{z}=p \frac{r}{h} \cdot\left\{\frac{1}{2}-\frac{1}{2} \frac{h}{r}\right\}
\end{aligned}
$$

From this it is concluded that for $h / r \approx$ constant, the path of stress is straight, since $\sigma_{r} / \sigma_{t}=$ constant and $\sigma_{r} / \sigma_{z}=$ constant. Using the relations from section 5.5, which applies for isotropic material properties, to relations (A 5.1.6), (A 5.1.7) and (A 5.1.8) for the components of the natural strain it is found that

$$
\begin{aligned}
& \varepsilon_{r}=-\frac{3}{4} \frac{\mathrm{pr}}{\mathrm{Hh}} \\
& \varepsilon_{t}=\frac{3}{4} \frac{\mathrm{pr}}{\mathrm{Hh}} \\
& \varepsilon_{z}=0
\end{aligned}
$$

The increase of the cavity volume of the cylinder $\Delta V$ is described by

$$
\Delta V=V_{c}\left(e^{\left(2 \varepsilon_{t}+\varepsilon_{z}\right)}-1\right)
$$

Substituting (A 5.1.9), (A 5.1.10) and (A 5.1.11) into (A 5.1.12), rewriting and neglecting the second order terms results in

$$
\Delta V=\frac{3}{2} \frac{p r}{H h} \cdot V_{c}
$$

According to the definition of the systolic left ventricular capacitance $c_{i v}^{i s}$ 


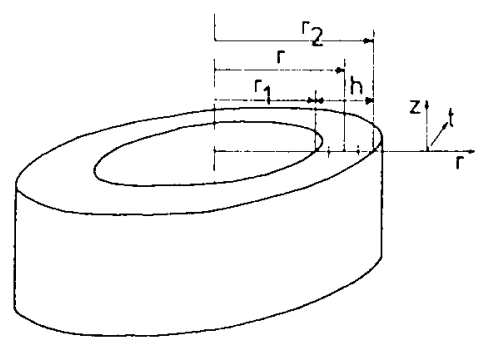

Figure A 5.1 .

A cylinder consisting of isotropic material.

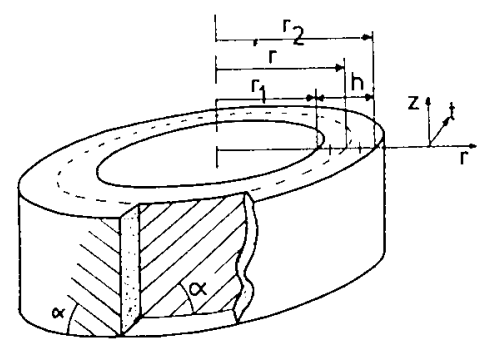

Figure A 5.2.

A cylinder composed of two concentric, fitting subcylinders, each consisting of anisotropic material as described in section 5.6. The angle indicates the fiber direction. 
for isotropic material properties it holds

$$
c_{l v}^{i s}=\frac{3}{2} \frac{V_{C} r}{H h}
$$

A 5.2. Capacitance of the left ventricle, consisting of anisotropic material, $\left(C_{\text {IV }}^{\text {an }}\right.$ fig. A 5.2)

As described in section 5.6 the anisotropic material is assumed to be incompressible and to consist of a fiber structure embedded in a soft, incompressible material. In a cylinder with center-wall radius $r$ and wall thickness $h$ the equilibrium of axial and tangential forces are leading to relations between stresses and internal pressure $p$. The equilibrium in axial direction results in

$$
\sigma_{z}=\frac{p r}{h}\left[\frac{1}{2}-\frac{1}{2} \frac{h}{r}+\frac{1}{8} \frac{h^{2}}{r^{2}}\right]
$$

The equilibrium in tangential direction results in

$$
\sigma_{t}=\frac{p r}{h}\left[1-\frac{1}{2} \frac{h}{r}\right]
$$

In the derivation of equations (A 5.2.1) and (A 5.2.2) $\sigma_{z}$ and $\sigma_{t}$ are assumed to be constant in the wall of the cylinder. In this case it holds for the radial stress $\sigma_{r}$

$$
\frac{d \sigma_{r}}{d r_{c}}=\frac{\sigma_{t}}{r_{c}}
$$

Since $\sigma_{t}$ is assumed to be constant, $\sigma_{r}$ can be derived by integration

$$
\sigma_{r}=\sigma_{t} \ln \left[\frac{r_{2}}{r_{c}}\right]
$$

Neglecting the second order terms with $\left(\frac{h}{r}\right)^{2}$ and averaging $\sigma_{r}$ over the wall thickness results in the relations (A 5.1.6), (A 5.1.7) and (A 5.1.8) mentioned before. The shear stresses in this coordinate system are zero, therefore the $r$-, $t$ - and $z$-directions are the principal directions of the stress tensor. Applying the constitutive relations of the anisotropic material to 
these stresses results in insolvability of the related strain, since in that material at least two principal stresses have to be equal. Therefore, as in the heart, different fiber orientations are assumed to exist in the wall of the cylinder. The simplest configuration which gives a solution, is a double layer model. In both layers of equal thickness the fibers are parallel with the wall of the cylinder. In one layer the fibers make an angle a with the equatorial plane, whereas in the other layer the fibers make an opposite angle with that plane. Because of the symmetry in the fiber orientations the system of both layers satisfies the requirement that the principal directions of the stress are the $r-$, $t$ - and $z$-directions. Since the fibers are supposed to be parallel to the wall the radial stress or equals the hydrostatic pressure in the soft, incompressible material. The stress, borne by the fibers, is obtained by subtraction of this hydrostatic pressure from the components of stress in the relations (A 5.1.7) and (A 5.1.8). For the tangential and axial components of this stress than it holds

$$
\begin{aligned}
& \sigma_{d t}=\frac{p r}{h} \\
& \sigma_{d z}=\frac{p r}{2 h}
\end{aligned}
$$

The angle $\alpha$ is determined by the equation

$$
\frac{\cos ^{2} a}{\sin ^{2} a}=\frac{\sigma_{d t}}{\sigma_{d z}}
$$

Substituting $(A 5.2 .5)$ and $(A 5.2 .6)$ into (A 5.2.7) results into

$$
a=\arctan \left(\frac{1}{2} \sqrt{2}\right)=35^{\circ}
$$

This angle $a$ is independent on the distending of the cavity volume of the cylinder. Therefore, during inflating the cylinder, the radius of the mid-wall and the height of the cylinder are equally straining, resulting in

$$
\Delta V=V_{c} \cdot\left(e^{3 \varepsilon_{1}}-1\right)
$$

where $\varepsilon_{1}$ is the natural strain of the fibers as well as of the radius and height. The relation between stress $\sigma_{1}$ in the fibers and stress $\sigma_{\mathrm{dt}}$ is 


$$
\sigma_{d t}=\sigma_{1} \cos ^{2} c_{1}
$$

Combining relation (A 5.2.5), (A 5.2.10), (A 5.2.9) with $\sigma_{1}=H \cdot \varepsilon_{1}$ and $\cos ^{2} a_{a}$ $=\frac{2}{3}$ results into following expression for the capacitance:

$$
c_{7 v}^{\text {an }}=\frac{9}{2} \frac{v_{c} r}{H h}
$$


Appendix 6.1

Conputation of the reflection coefficient in a bifurcation (see fig. 6.52

For the internal radius in a bifurcation it holds according to relation (6.2.14)

$$
r_{\text {prox }}^{s}=r_{\text {dist } 1^{s}}+r_{\text {dist }} 2^{s}
$$

where $r_{\text {prox }}, r_{\text {dist } 1}$ and $r_{\text {dist } 2}$ are the radii of the proximal and two distal branches, respectively.

$S$ is an exponent which was experimentally found to be $2.55 \pm .03$ (mean $\pm \mathrm{SE}$; fig. 6.6). In case of symmetry, i.e.

$$
r_{\text {dist } 1}=r_{\text {dist } 2}=r_{\text {dist }}
$$

relation (A 6.1 ) can be transformed into

$$
\frac{r_{\text {dist }}}{r_{\text {prox }}}=2^{-\frac{1}{s}}
$$

In the analysis reflection is considered to be a result of unnatched characteristic impedances 1 ) of the branches in a bifurcation. For a sine wave the characteristic impedance of the proximal branch is

$$
Z_{\text {o prox }}=\sqrt{\frac{z}{y}}=\frac{1}{\pi r_{\text {prox }}^{2}} \sqrt{\frac{\rho K}{F\left(a_{\text {prox }}\right)}}
$$

where $z \quad=$ impedance of the vessel per unit of length

$y=$ adnittance of the vessel per unit of length

$a_{\text {prox }}=r_{\text {prox }} \sqrt{\rho \omega / n}$ (according to Womersley $\left.{ }^{2}\right)$ )

$F(a)=J_{2}(a \sqrt{-i}) / J_{0}(a \sqrt{-i})$ with $i=\sqrt{-1}$ and $J_{0}, J_{2}$ Bessel functions ${ }^{3)}$

Assuming an equal $K$ value for the distal branches, one finds for the characteristic impedance of the distal branches

$$
z_{\text {odist }}=\frac{1}{\pi r_{\text {dist }}^{2}} \cdot \sqrt{\frac{\rho k}{F\left(a_{\text {dist }}\right)}}
$$


with $a_{\text {dist }}=r_{\text {dist }} \sqrt{\text { ow/ } / 1}$.

The reflection coefficient in the bifurcation is

$$
R_{C}=\frac{Z-Z_{0 \text { prox }}}{Z+Z_{0 \text { prox }}}
$$

where $Z$ represents the distal loading impedance of the proximal branch, being the two distal characteristic impedances in parallel:

$$
z=\frac{1}{2} z_{0} \text { dist }
$$

substitution of (A 6.4), (A 6.5) and (A 6.7) into (A 6.6) results in

$$
R_{c}=\frac{q-1}{q+1} \text { with } q=\frac{1}{2}\left(\frac{r_{\text {dist }}}{r_{\text {prox }}}\right)^{2} \sqrt{\frac{F\left(a_{\text {prox }}\right)}{F\left(\frac{r_{\text {dist }}}{r_{\text {prox }}} \cdot a_{\text {prox }}\right)}}
$$

After substitution of (A 6.3) into (A 6.8) for $R_{C}$ a complex function is found depending on $a_{\text {prox }}$ and $s$. The results of the computations are shown in fig. A 6.1 .

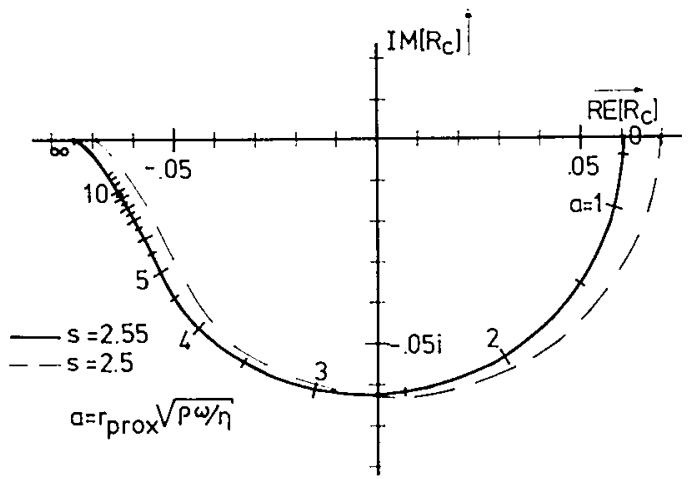

Figure A 6.1.

The reflection coefficient at a bifurcation of a coronary artery in the complex plane as a function of rprox $\sqrt{\rho \omega / \eta}$, where prox $=$ radius of the proximal branch; $0=$ the density of the blood; $\omega=$ angular frequency $\quad ; n=$ viscosity of the blood. 
For an internal diameter of the coronary artery of $1.5 \mathrm{~mm}$ and the highest frequency assumed to be present in the signal $(50 \mathrm{~Hz})$ the largest value of a prox is approximately 14. Substituting for $s$ the experinentally determined value 2.55 gives a modulus of the reflection coefficient smailer than $6.7 \%$ for all significant values of aprox (fig. A 6.1).

To show the dependency on the value of $s$, also the curve of $R_{C}$ for $s=2.5$ is shown. In this case the function shifts in the complex plane, and consequently for certain frequencies $\left|R_{c}\right|>6.7 \%$. Obviously, the experimentally determined value of the exponent $s$ is very close to the optimal value, which explains why during diastole minimum reflections appear in the coronary vasculature.

Appendix 6.2

\section{Computation of the cross-sectional stiffness $K$}

The cross-sectional stiffness $k$ is computed from the wave front velocity. The velocity is defined as the ratio of the distance between the sites of pressure measurement and the time interval between the appearance of the pressure step at the proximal and next at the distal site of measurement. The signals were compared at $25 \%$ of the maximum pressure change. In a computer simulation a pressure step of the following form was supposed to be measured at the first site of pressure measurement

$$
p_{1}(t)=\frac{1}{\tau} \sqrt{\frac{2}{\pi}} \int_{0}^{t} \exp \left(\frac{-t^{2}}{2 \tau^{2}}\right) d t
$$

where $\tau$ determines the rise time.

In absence of reflections the transfer function $F_{1.2}(\omega)$ of the vessel segment is

$$
F_{1.2}(\omega)=e^{\beta(\omega) l}
$$

where 


$$
B(\omega)=-i \sqrt{-2 y}=-i \omega \sqrt{-\frac{\rho}{K} \frac{J_{0}\left(r \sqrt{-i \omega \frac{\rho}{n}}\right)}{J_{2}\left(r \sqrt{-i \omega \frac{\rho}{n}}\right)}}
$$

Fourier transformation of $p_{1}(t)$, followed by multiplication by the transfer function $F_{1.2}(\omega)$ and inverse fourier transformation, results in a function $p_{2}(t)$ which is expected to represent the pressure registration at the distal site. The wave front velocity $v_{w f}$ was determined according to the above mentioned definition. According to these calculations $K$ appears to be equal to

$$
K \approx 1.25 \rho v_{w f}^{2}
$$

in the case of an internal vessel radius of $1 \mathrm{~mm}$ and a pressure rise time of $10 \mathrm{msec}$, which were average values in our experiments.

\section{Appendix 6.3}

\section{Computation of the internal diameter of the vessel segment in vivo}

Assume a tube with length (1), an internal radius at the entrance $\left(r_{e}\right)$ and an uniform flow leakage along the tube by many small sidebranches. Then the volume flow at a distance $x$ from the entrance $q(x)$ will equal:

$$
a(x)=q_{e}(1-\lambda \stackrel{x}{T})
$$

with $\lambda=$ leakage fraction.

If there is no flow leakage between the flowprobe and the first site of pressure measurement, $a_{e}$ equals the coronary artery flow measured. Through relation (6.2.12) relation (A 6.12 ) gives for the internal radius $r$ of the vessel

$$
r(x)=r_{e}\left(1-\lambda \frac{x}{T}\right)^{\frac{1}{2.55}}
$$

The pressure drop per unit of length then becomes

$$
-\frac{d p}{d x}=q_{e} \frac{8 \eta}{\pi r_{e}^{4}}\left(1-\lambda \frac{x}{T}\right)^{\left(1-\frac{4}{2.55}\right)}
$$


The total pressure drop can be expressed as

$$
i p=\int_{0}^{1}-\frac{d p}{d x} d x=q_{e} \frac{8 n]}{\pi r_{e}^{4}} \frac{1-(1-\lambda)^{0.431}}{0.431 \lambda}
$$

The assumption that the cross-sectional area of the vessel is proportional to the internal pressure results in the relation

$$
r_{e}^{2}=\left(1+\frac{p}{k}\right) r_{e o}^{2}
$$

where $r_{\mathrm{eo}}=$ the extrapolated value of the vessel radius with $p=0$. Hence the radius at the entrance during diastole $\left(r_{d}\right)$ is given by

$$
r_{d}{ }^{4}=\frac{q_{d}-q_{s}\left(\frac{1+p_{d / k}}{1+p_{s / k}}\right)^{2}}{\Delta p_{d}-i p_{s}} \cdot \frac{1-(1-\lambda)^{0.431}}{0.431 \lambda} \cdot \frac{8 n 1}{\pi}
$$

\section{Literature}

1. Pender, HcIlwain: Electrical engineers handbook. New York: Wiley, New York 4 th ed. $5,22-26(1954)$.

2. Womersley, J.R.: Method for the calculation of the velocity, rate of flow and viscous drag in arteries when the pressure gradient is known. J Physiol (London) 127, 553 - 563 (1955). 


\section{SAMENVATTING}

Dit proefschrift beschrijft een wiskundig model van de mechanische werking van de linker ventrikel, warin tevens de coronaire cirkulatie is opgenomen. Het model bestaat uit een aantal deelmodellen, welke deelsystemen beschrijven, zoals de geometrie en mechanica van de linker ventrikel, de mechanische eigenschappen van het spiermateriaal met inbegrip van de spierkontraktie, de hemodynamische belasting van de linker ventrikel, de grote coronaire arterieën en de coronaire mikrocirkulatie. De beschrijving van een deelmodel is steeds gebaseerd op de fysische eigenschappen van het betreffende deelsysteem, waardoor de gekozen parameters een fysische betekenis hebben. De parameterwaarden zijn zo veel mogelijk verkregen uit in vivo en in vitro onderzoekingen, welke uitgevoerd werden ter bestudering van het betreffende deelsysteem. Na samenstelling van het model uit de deelmodellen verd een hartcyclus nagebootst (fig. 10.1). Ofschoon enkele duidelijke verschillen met de normale hartcyclus gevonden werden, hadden veel grootheden een voorkomen, dat goed overeenkwam met dat in dierexperimenten onder vergelijkbare omstandigheden. Dit betrof het tijdsverloop van de stroomsterkte en de druk in de aorta, de hoogte van de linker ventrikeldruk, de ejektie fraktie en, kwalitatief, het gedrag van de stroomsterkte in de coronaire arterieën. Dit geeft aan, dat de opzet van het model redelijk juist is.

Vervolgens werden enkele parameterwaarden zodanig gewijzigd, dat de resultaten van het model beter overeenkwamen met de resultaten van dierexperimenten. Deze vorm van parameterschatting heeft echter enkele nadelen. Verschillen tussen experimentele resultaten en nabootsingen kunnen weggewerkt worden door waardeverandering van foutieve parameters, waardoor elders in het model nieuwe verschillen ontstaan. Verder kunnen systematische fouten in het model verdoezeld worden. Aldus ontstane fouten kunnen de geldigheid en bruikbaarheid van het model ernstig aantasten. Daarom is het aantal parameters, waarvan de waarde gewijzigd is, laag gehouden en zijn opzettelijk enkele minder belangrijke verschillen gehandhaafd. De aanpassing van de parameterwaarden was gemakkelijk, aangezien hun fysische betekenis bekend is. De aanpassingen betroffen parameters, welke in verband staan met het tijdsverloop van de aktivatie van de hartspier en het transmurale verloop (= verloop als funktie van de afstand tot de binnenwand van de ventrikel) van de spiervezelrichting, de sarcomeerlengte en het volume van het coronaire vaatbed. Vervolgens werd een tweede hartcyclus nagebootst (fig. 10.2), waarvan de resultaten goed 
overeen bleken te komen met dierexperimentele bevindingen. Hierbij werd gelet op het tijdsverloop van de linker ventrikeldruk, de druk en stroomsterkte in de aorta, de afmetingen van de linker ventrikel en de stroomsterkte in de coconaire arteriëen.

Hierna werden met behulp van het model afwijkingen in de normale hartwerking nagebootst door waardeverandering van de geschikte parameters. Het vinden van de geschikte parameters werd vergemakkelijkt door het feit, dat de meeste parameters een fysische en fysiologisch interpreteerbare betekenis hebben. De resultaten van de nabootsingen van verschillende afwijkingen in de normale hartwerking stemden redelijk goed overeen met dierexperimentele bevindingen, waarbij gelet werd op de linker ventrikeldruk, de druk en de stroomsterkte in de aorta, de stroomsterkte in de coronaire arterieën en de transmurale verdeling van de coronaire doorstroming in de ventrikelwand. Er werden evenwel enkele afwijkingen gekonstateerd. De oorzaken van deze verschillen met het dierexperiment kunnen voor een deel toegeschreven worden aan het in het model buiten beschouwing laten van de rechter ventrikel en enkele andere mechanisch van belang zijnde strukturen in het hart, onzekerheden in de fysische eigenschappen van het hartspiermateriaal en de coronaire mikrocirkulatie, en de vereenvoudiging van de aortaklep.

Uit de nabootsingen bleek, dat de torsiehoek van de linker ventrikel erg gevoelig was voor transmurale verschillen in mechanische belasting van het hartspiermateriaal. Voor zover bekend, is deze torsiehoek nog nooit in het dier gemeten. Uit de modelstudie kan gekonkludeerd worden, dat bij bestudering van de mechanica van de linker ventrikel de meting van deze hoek een waardevol hulpmiddel kan zijn. Deze konklusie wordt toegelicht aan de hand van het volgende voorbeeld. Als gevoig van een ernstige stenose in een coronaire arterie verslechtert de mechanische werking van het hart. Uit een nabootsing van een hartcyclus onder deze omstandigheden blijkt, dat de coronaire doorstroming het eerst ontoereikend wordt in de binnenste, subendocardiale, lagen van de linker ventrikel. Dit verschijnsel wordt bevestigd door dierexperimenten. Door meting van het tijdsverloop van de torsiehoek tijdens de samentrekking van het hart kan men meer te weten komen over de lagen, die verantwoordelijk zijn voor de afname van de pompwerking. Uiteraard is het hier gepresenteerde model van nut bij de interpretatie van veranderingen in de torsiehoek.

Bij aanpassing van het transmurale verloop van de spiervezelrichting is de hypothese gesteld, dat transmurale verschillen in trekspanning in de 
hartspiervezels gering zijn. Deze hypothese leidt tot een konsept van de bouw van het hart, welk ongevoelig blijkt voor verschillen in hemodynamische belasting. Transmurale verschillen in de grootheden, die in verband staan met de mechanische belasting en de coronaire doorstroming blijven gering. Hieruit kan gekonkludeerd worden, dat het hart, inklusief de rechter ventrikel, mogelijk zodanig is opgebouwd, dat de voornoemde hypothese geldig is voor het gehele hart.

In deze studie werd aangenomen, dat het mechanische gedrag van het hartspiermateriaal anisotroop is. Het materiaal werd opgevat als een vezelstruktuur, die kan samentrekken en ingebed is in een zacht, onsamendrukbaar materiaal. Bij toepassing van isotrope materiaaleigenschappen bleken de resultaten van het model slecht overeen te stemmen met dierexperimentele bevindingen, de ventrikel vertoonde een te stug gedrag. Hieruit is gekonkludeerd dat modellen van de hartspier, welke gebaseerd zijn op isotrope wandeigenschappen, niet realistisch zijn.

Een algemene konklusie is, dat het model een nuttig hulpmiddel is bij de begripsvorming over het dynamisch gedrag van de linker ventrikel en de coronaire doorstroming. De hemodynamica, veranderingen in de afmetingen tijdens de hartcyclus en het transmurale verloop van grootheden, die in verband staan met de mechanische belasting en de coronaire doorstroming, zijn kwantitatief met elkaar in verband gebracht. Deze kwantificering opent mogelijkheden tot het bestuderen van relatief ingewikkelde, vergaande gevolgen van verscheidene ingrepen en afwijkingen van de normale situatie. Deze algemene konklusie leidt tot een aantal voorgestelde toepassingen van het model, waaronder:

- nabootsing van ingrepen in de hartcyclus

- bestudering van deelsystemen van het hart in vivo

- bestudering van het transmurale verloop in de ventrikelwand van grootheden, die in verband staan met de mechanische belasting en de coronaire doorstroming

- vorming van een begrip van klinisch gevonden afwijkingen in de hartwerking

- toepassing in het onderwijs, waarbij begripsvorming voorop staat. 


\section{LEVENSBERICHT}

9 april 1947

geboren te lijchen.

$1959-1964$

HBS-b gevolgd aan het Canisius College te l!ijmegen.

$1964-1971$

Elektrotechniek gestudeerd aan de Technische Hogeschool Eindhoven.

1 februari 1971 in dienst getreden als wetenschappelijk medewerker van de afdeling Werktuigbouwkunde, Technische Hogeschool Eindhoven.

1 januari 1972 In dienst getreden van de Stichting Zuiver Hetenschappelijk Onderzoek, gestationeerd aan de afdeling Werktuigbouwkunde, Technische Hogeschool Eindhoven.

1 januari 1976 In dienst getreden als wetenschappelijk medewerker van de capaciteitsgroep Biofysica van de Rijksuniversiteit Limburg. 
NAWOORD

De studie, die aan dit proefschrift ten grondslag ligt, is begonnen als een projekt binnen de werkgemeenschap hartfunktie van de Stichting voor Medisch Wetenschappelijk Onderzoek (FUNGO). Prof. dr. R.S. Reneman was de projektleider. Ik was gestationeerd in de werkgroep voor biomedische techniek, die onder leiding stond van prof. dr. P.C. Veenstra aan de Technische Hogeschool Eindhoven. Dit proefschrift is voltooid binnen het kader van het deelprojekt Myocard onder leiding van prof. dr. R.S. Reneman, aan de Rijksuniversiteit Limburg.

Hierbij dank ik eenieder die bijgedragen heeft, in welke vorm dan ook, aan de totstandkoming van dit proefschrift.

Prof. dr. R.S. Reneman, Rob, heeft mij kennis en toepassing van fysiologische principes bijgebracht. Bovendien heeft hij mij uitstekend de weg gewezen in de wereld van het cardiovasculaire onderzoek. Prof. dr. P.C. Veenstra verplaatste zich steeds zeer snel in de aktuele problematiek van het onderzoek en gaf vaak nieuwe, waardevolle richtingen aan. Dr. P. Janssen makte het mogelijk noodzakelijke dierexperimenten uit te voeren in het laboratorium van Janssen Pharmaceutica te Beerse. Bij het tot stand komen van dit proefschrift heb ik zeer waardevol kommentaar gekregen van prof. dr. J.D. Laird en dr. N. Westerhof, waarmee voor mij het belang van kritische referenten, die buiten het eigenlijke onderzoek staan, nog eens benadrukt is.

De werkgroep biomedische techniek bleek een uitstekende voedingsbodem voor multidisciplinair onderzoek. De bijna dagelijke wetenschappelijke (en andere) discussies met Jos Spaan waren steeds zeer stimulerend. Aan Anton van Steenhoven, Jan Oomens, Theo van Duppen, Jack Couwenberg en Frans Karel van Hely heb ik steeds prettige kollega's gehad, die elk op hun gebied, steeds bereid waren zich voor mijn werk in te zetten. Nico Touwen heeft me vaak uit de problemen van computer programmering geholpen. Zijn geologische stiptheid is van groot nut gebleken bij het systematisch opzetten van het uiteindelijke computer programma. Het afstudeerwerk van Bert van Huygevoort heeft de aanzet gegeven tot de huidige aanpak van de mechanica van het hart. Jan Smit wil ik bedanken voor zijn betrokkenheid bij de menselijke kanten van het onderzoek en de onderzoeker.

Ruud Krueger, Willie van Gerven, Jacques Lambregts en Cees Verlaan voerden de noodzakelijke dierexperimenten uit. De leden van de capaciteitsgroen Biofysica hebben mij de gelegenheid gegeven mij te onttrekken aan alle organisatorische verplichtingen, zodat ik dit proefschrift kon voltooien. Arnold Hoeks bedank ik voor het waarnemen van een commissie-voorzitterschap gedu- 
rende deze tijd. Dick Slaaf stelde steeds zijn tijd beschikbaar op de kritieke momenten. De grote hoeveelheid typewerk is vrijwel geheel verricht door Rosy Borgman.

Zoals bij het schrijven van zovele proefschriften het geval is, is ook bij het vervaardigen van dit proefschrift een aanslag op het privéleven gepleend. Jacqueline, je hebt mijn soms totale geestelijke afwezigheid tijdens mijn lijfelijke aanwezigheid moeten verdragen. Op de kritieke momenten ben je steeds in de bres gesprongen door in je eentje voor het gez in te zorgen en tevens delen van dit proefschrift uit te typen. 